\title{
A Detailed Study of Axon Initial Segment Maturation and Structural Organization by Fluorescence Microscopy
}

\author{
Dissertation \\ for the award of the degree \\ "Doctor rerum naturalium" \\ of the Georg-August-Universität Göttingen \\ within the doctoral program GGNB \\ of the Georg-August University School of Science (GAUSS) \\ submitted by \\ Melanie Dannemeyer \\ from Brake (Unterweser), Lower Saxony
}

Göttingen 2015 

Members of the Thesis Committee:

Prof. Dr. J. Enderlein (Reviewer)

3rd Institute of Physics - Biophysics, Faculty of Physics

Georg-August-University Göttingen

\section{Dr. A. Neef (Reviewer)}

Bernstein Center for Computional Neuroscience Göttingen

MPI for Dynamics and Self-Organization

Prof. Dr. K. Tittmann

Department of Bioanalytics, Faculty of Biology and Psychology

Georg-August-University Göttingen

Prof. Dr. Luis A. Pardo

Department of Molecular Biology of Neuronal Signals

Max Planck Institute for Experimental Medicine

\section{Dr. Dieter Klopfenstein}

3rd Institute of Physics - Biophysics, Faculty of Physics

Georg-August-University Göttingen

\section{Dr. Florian Rehfeldt}

3rd Institute of Physics - Biophysics, Faculty of Physics

Georg-August-University Göttingen

Date of oral examination: 25.01.2016 


\section{Affidavit}

Hereby, I declare that the presented thesis has been written independently and with no other sources and aids than quoted.

Göttingen, 26.11.2015 


\section{Contents}

\begin{tabular}{lr}
\hline Abstract & 1
\end{tabular}

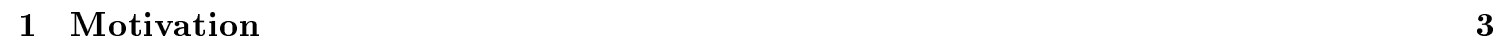

\begin{tabular}{|rr}
\hline 2 & Introduction \\
\hline
\end{tabular}

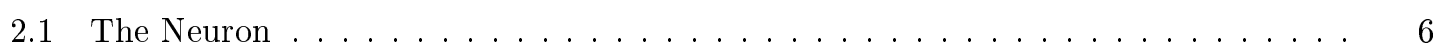

2.1 .1 The Axon Initial Segment $\ldots \ldots \ldots \ldots \ldots$

2.2 The cytoskeletal protein spectrin . . . . . . . . . . . . . . . . . . 9

2.3 The adapter protein ankyrin . . . . . . . . . . . . . . . . . . . 10

2.4 Voltage-gated sodium channels . . . . . . . . . . . . . . . . . . . . . 12

2.5 The quivering mutation . . . . . . . . . . . . . . . . . . 14

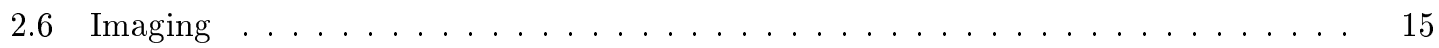

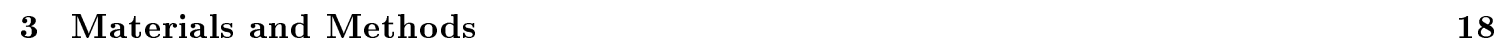

3.1 Cell culture . . . . . . . . . . . . . . . . . . . . . . . 18

3.2 Genotyping . . . . . . . . . . . . . . . . . . . . . 19

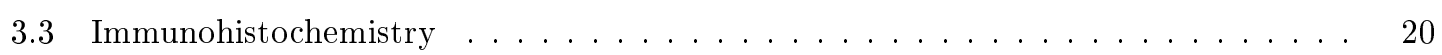

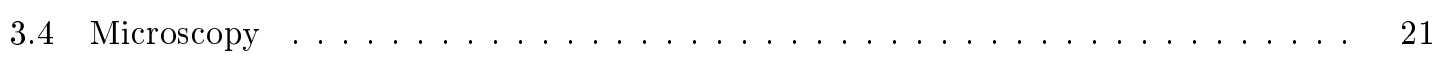

$3.4 .1 \quad$ Wide-field microscopy $\ldots \ldots \ldots \ldots \ldots \ldots \ldots \ldots \ldots \ldots$

3.4 .2 STORM microscopy . . . . . . . . . . . . . . . . . . . 22

3.5 Data Analysis . . . . . . . . . . . . . . . . . . . . 25

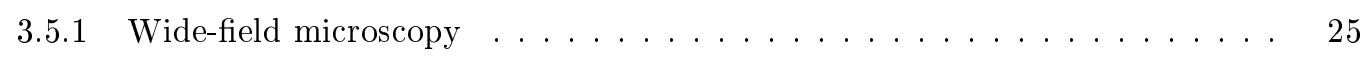

$3.5 .2 \quad$ STORM microscopy $\ldots \ldots \ldots \ldots \ldots \ldots \ldots \ldots \ldots$

4 Axon initial segment maturation in hippocampal neurons 29

4.1 Results . . . . . . . . . . . . . . . . . . . . . . . . . . 29

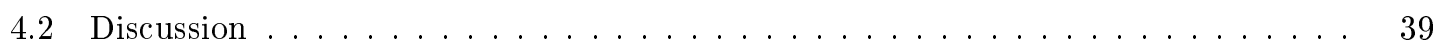

5 Structural organization of axon initial segment components in hippocampal $\begin{array}{ll}\text { neurons } & 43\end{array}$

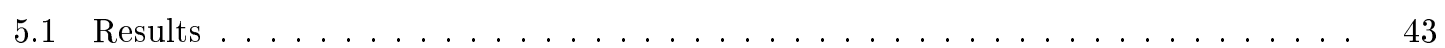

5.2 Discussion $\ldots \ldots \ldots \ldots \ldots \ldots \ldots \ldots$

6 Axon inital segment maturation in hippocampal neurons derived from $q v^{3 J}$ $\begin{array}{ll}\text { mutant mice } & 54\end{array}$

6.1 Results . . . . . . . . . . . . . . . . . . . . . . . 54 


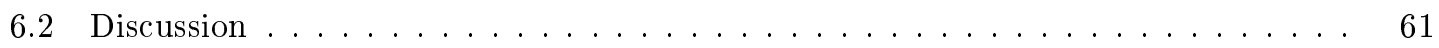

7 Structural organization of axon initial segment components in hippocampal $\begin{array}{ll}\text { neurons derived from } q v^{3 J} \text { mutant mice } & 63\end{array}$

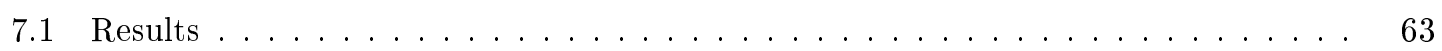

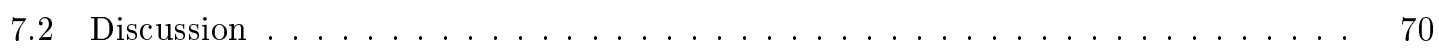

\begin{tabular}{lll}
\hline 8 & Conclusion & 72
\end{tabular}

$\begin{array}{lll}9 & \text { Appendix A: Experimental procedure } & 76\end{array}$

$9.1 \quad$ The specificity of immunohistochemistry $\ldots \ldots \ldots \ldots \ldots$. . . . . . . . 76

$9.1 .1 \quad \beta$ IV-spectrin antibodies $\ldots \ldots \ldots \ldots \ldots \ldots \ldots$

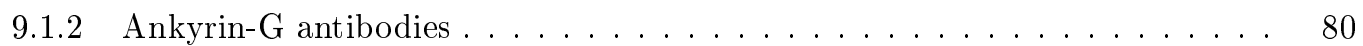

9.1 .3 Subtype $\mathrm{Na}_{\mathrm{v}} 1.6$ antibodies $\ldots \ldots \ldots \ldots \ldots \ldots \ldots$. . . . . . . . 82

9.1 .4 Subtype $\mathrm{Na}_{\mathrm{v}} 1.2$ antibodies $\ldots \ldots \ldots \ldots \ldots \ldots$. . . . . . . . 84

\begin{tabular}{|l|}
\hline 10 Appendix B: Results \\
\hline
\end{tabular}

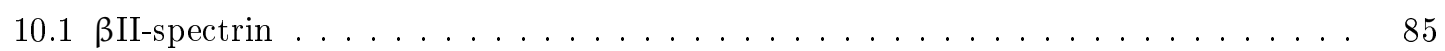

10.2 The structure of ankG targeted C-terminal in immature neurons . . . . . . . . . . 88

10.3 Details of voltage-gated sodium channel subtype $\mathrm{Na}_{\mathrm{v}} 1.2 \ldots$. . . . . . . . . . 91

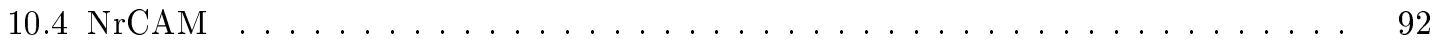

\begin{tabular}{|r}
\hline 11 Appendix C \\
\hline
\end{tabular}

11.1 Kolmogorov-Smirnov-Test $\ldots \ldots \ldots \ldots \ldots \ldots$

11.2 Antibodies . . . . . . . . . . . . . . . . . . . . 99

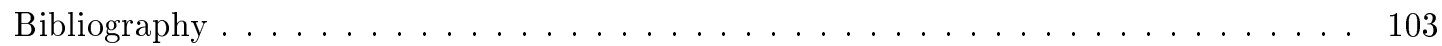

List of Figures . . . . . . . . . . . . . . . . . . . . . . . . . . 118

List of Tables . . . . . . . . . . . . . . . . . . . . . . . . . . . . 119

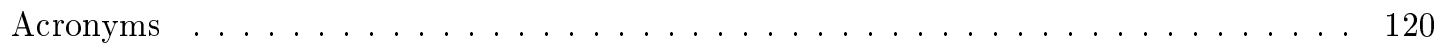

Acknowledgments . . . . . . . . . . . . . . . . . . . . 123

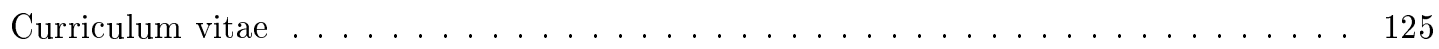




\section{Abstract}

The distribution and density of neuronal voltage-gated sodium channels (VGSCs) is important for neuronal excitability, maturation and plasticity. High density of VGSCs in the axon initial segment (AIS) depends on the cytoskeletal proteins $\beta I V-s p e c t r i n$ and ankyrin-G (ankG). Here, the density and structure of $\beta I V$-spectrin, $\beta$ II-spectrin, ankG and VGSCs in the AIS is studied with maturation. Neuronal cultures were prepared from hippocampus and immunofluorescence was imaged between 7 and 21 days in vitro with conventional wide-field and stochastic optical reconstruction microscopy (STORM).

At the earliest time point studied, DIV \%, $\beta$ II-spectrin is arranged in the AIS on a very regular structure. With maturation $\beta$ II-spectrin density decreases slightly, while the density of $\beta I V-$ spectrin increases in the AIS. Neuronal $\beta I V$-spectrin appears in two isoforms $\beta \operatorname{IV} \sum 1$ and $\beta \operatorname{IV} \sum 6$. Within this detailed study the density and structural organization of $\beta I V$-spectrin is examined in detail. The mean fluorescence intensity of $\beta I V$-spectrin labeled at its N-terminus is found on a constant level starting at $D I V$ 14. Based on the fact that N-terminal labeling targets $\beta I V \sum 1$, but not $\beta \operatorname{IV} \sum 6$, the mean fluorescence intensity stagnates within the second week of studied maturation and a prominent periodic organization is revealed, it is suggested that $\beta \operatorname{IV} \sum 1$ is highly enriched early on, arranged in the AIS on a very regular basis and functions as the main stabilizing $\beta I V-$ spectrin isoform. Contrariwise, C-terminal labeling of $\beta I V$-spectrin reports an increase of the mean fluorescence intensity over the whole three weeks of studied maturation. Moreover, at DIV 6 $\beta I V-s p e c t r i n$ is found on a very regular basis, whereas at DIV 14 a diffuse distribution is observed. Based on the fact that C-terminal labeling targets $\beta \operatorname{IV} \sum 1$ and $\beta \operatorname{IV} \sum 6$, the careful study allows to conclude that the detected increase of the mean fluorescence intensity and thus likely an increase of the density of $\beta I V$-spectrin density is due to an increase of $\beta \operatorname{IV} \sum 6$. Further, a diffuse $\beta I V$-spectrin structure in mature neurons suggests an irregular placement of $\beta \operatorname{IV} \sum 6$ in the existing regular structured cytoskeleton of actin-adducin rings. The adapter protein ankG mediates the contact between VGSCs and the underlying cytoskeleton. Its density is found to increase over the studied maturation period. AnkG is found organized prominently periodically in immature neurons. With maturation the periodic organization of ankG becomes less pronounced. Whereas previous studies simplify the observation by reporting the distance of ankG to the underlying network and the distribution of binding positions on a spectrin tetramer are the cause, not considering that these conditions are not only found in mature neurons, but also immature neurons, here a high quality study suggests non-periodic $\beta \operatorname{IV} \sum 6$ as a mediator for the increase of irregularity of ankG. In agreement with ankG, the density of VGSCs and specifically the densities of the subtypes $\mathrm{Na}_{\mathrm{v}} 1.2$ and $\mathrm{Na}_{\mathrm{v}} 1.6$ increase with maturation. Additionally, the data suggest an early expression of $\mathrm{Na}_{\mathrm{v}} 1.2$ 
(measured $D I V 6$ ) and a late expression of $\mathrm{Na}_{\mathrm{v}} 1.6$ (not detected at $D I V$ \%, but at $D I V 10$ ). Whereas, $\mathrm{Na}_{\mathrm{v}} 1.2$ is found proximal in immature and evenly distributed in mature neurons, is $\mathrm{Na}_{\mathrm{v}} 1.6$ found distal in mature neurons and evenly distributed in immature neurons. A developmental switch from one subtype to another is not found. VGSCs and specifically $\mathrm{Na}_{\mathrm{v}} 1.6$ are found (semi)periodic. The data obtained for $\mathrm{Na}_{\mathrm{v}} 1.2$ organization do not allow to draw a final conclusion, yet. The mouse strain $q v^{3 J}$ is a loss of function mutation in the $\beta I V-$ spectrin gene (Spnb4). A single inserted base (InsT6786) causes a frameshift at amino acid G2209 and a completely changed new 49-amino-acid C-terminal extension. ${ }^{1}$ Here, the effect of this mutation on the expression and structural organization of $\beta I I-$ spectrin, $\beta I V$-spectrin, ankG and VGSCs in the AIS is studied. At $D I V 7$ wildtype and mutant neurons are very similar with respect to density and periodicity of ankG, VGSCs and $\beta I I-s p e c t r i n$. However, only very little $\beta I V$-spectrin is observed in mutant $q v^{3 J}$ neurons at $D I V 7$ and is not detectable when labeling DIV 14 neurons. The density and regularity of $\beta I I-$ spectrin is not altered, suggesting that it has no role within a rescue mechanism for $\beta I V$-spectrin absence. In contrast to the maturation in wildtype no increase of the ankG and VGSCs density is observed in $q v^{3 J}$ mutant neurons. AnkG is found at a constant density and arranged strongly periodic between DIV 11 - 24. This supports its binding to $\beta I I-s p e c t r i n$ and suggests an absence of $\beta \operatorname{IV} \sum 6$ as an irregular cytoskeletal binding partner. The VGSC density in mutant $q v^{3 J}$ neurons decreases progressively in the three weeks of studied maturation. A detailed analysis suggests that $\mathrm{Na}_{\mathrm{v}} 1.2$ channels are not stabilized and constantly decrease in density with maturation, whereas $\mathrm{Na}_{\mathrm{v}} 1.6$ channels are rare at all ages, due to a not fully functional recruitment mechanism of VGSCs by the ankG- $\beta I V$-spectrin complex. Furthermore, the constant ankG density, but a consecutive decrease of VGSC density, suggests a direct relation between VGSCs and $\beta I V-$ spectrin. The disruption of the complete mechanism is suggested to be mainly due to a disrupted $\beta \operatorname{IV} \sum 1$, an isoform suggested to function as a stabilizer, and the complete absence of $\beta \operatorname{IV} \sum 6$, an isoform suggested to function as a recruiter for VGSCs in neuronal maturation.

To conclude, $\beta I V$-spectrin is incorporated in the existing $\beta \mathrm{II}$-spectrin grid, $\beta \mathrm{IV} \sum 1$ periodic and $\beta I V \sum 6$ non-periodic. The ring-like organization of proteins along the AIS does not depend on $\beta I V$-spectrin. The C-terminal mutation $q v^{3 J}$ causes a complete loss of $\beta I V$-spectrin. The $\beta I V-$ spectrin C-terminal tail is essential for its incorporation and stabilization. $\beta I V$-spectrin is a key component in ankG and VGSC recruitment. BIV-spectrin is crucial for VGSC stabilization. In addition, ankG stabilization depends strongly on $\beta \mathrm{II}$-spectrin. 


\section{Motivation}

Neurons in the brain exchange information in a digital code, by means of brief electrical pulses, called action potentials. The action potential (AP) is generated in the axon initial segment (AIS).2] 3 The AIS underlies a complex maturation progress and a highly specialized composition, including a unique structural organization, which allows the enrichment of voltage-gated sodium channels (VGSCs), lowering its threshold and giving it the functionality as the site of AP initiation. 4 [5 60 AIS dysfunctions, mediated by composition alterations, have been linked to multiple neuronal disorders, such as epilepsy, Alzheimer's and schizophrenia. ${ }^{[9] 6}$ In order to understand AIS dysfunction and identify abnormalities in composition, the maturation and structure of the AIS needs to be fully understood. Thereby, it is of interest to establish a detailed model of the AIS structure, and monitor and quantify concurrent maturation of cytoskeletal proteins and the functional VGSCs. The two methods used in this thesis, wide-field and stochastical optical reconstruction microscopy (STORM) imaging, allow (i) a semi-quantification of AIS protein expression levels with AIS maturation and (ii) imaging of structural organization. The goal of the thesis is to present a model of the AIS, showing its full complexity and leading towards a better understanding of the AISs functionality and observed phenotypes during diseases.

Previous work within the Bernstein Group "Biophysics of neural information encoding" focused on the maturation of electrophysiological properties in hippocampal neurons. Neurons were monitored between the age of DIV 6 to 55 in vitro. The somatic AP waveform was analyzed to extract parameters thought to reflect the functional properties and densities of VGSCs in the axon (AP threshold, voltage dependence of the initial rate of change of the membrane potential $\left(d^{2} V / d t d V\right)$ ) and in the soma (peak rate of rise of the membrane potential). Axonal and somatic components changed significantly within the first two weeks and stabilized at the end of the third week, suggesting that the neurons reached their mature stage. 1 As the functionality of the AIS has been shown to depend on the enrichment of VGSCs, 4] [5] [6] [8 it is of interest to monitor simultaneously the compositional and structural maturation of the AIS. Here, it is focused on monitoring VGSC density, especially the subtypes $\mathrm{Na}_{\mathrm{v}} 1.2$ and $\mathrm{Na}_{\mathrm{v}} 1.6$, until the neurons reach the their mature stage. Further, it is of interest to look at the densities of cytoskeletal proteins, such as $\beta I V$-spectrin and ankyrin-G (ankG), which have been shown as function as linker and adapter proteins for proper VGSC placement and thus play a role in AIS maintenance (Chapter: 4). Besides protein densities, a deeper insight is gained by a structural study.

\footnotetext{
${ }^{1}$ Previous work: Electrophysiology measurements on wildtype neurons were performed by Elinor Lazarov, Hebrew University of Jerusalem, Israel during her PhD thesis. Manuscript: (1) Elinor Lazaorv, Melanie Dannemeyer, Anja Huss, Michael Gutnick, Fred Wolf, Andreas Neef "Functional and structural maturation of the axon initital segment in cultured hippocampal neurons."
} 
In January 2013 the lab of Xiaowei Zhuang reported the existence of a highly regular cytoskeleton and associated VGSCs in the AIS of rat hippocampal neurons. Based on their high resolution images obtained by STORM microscopy, a model of the structural organization of AIS proteins has been established. The work was performed based on a previous model of the actin-adducinspectrin-ankyrin network, imaged underneath the membranes of swollen erythrocytes. ${ }^{10}$ Xiaowei Zhuangs lab studied actin, adducin, $\beta$ II-spectrin, $\beta I V-s p e c t r i n$, ankyrin-B (ankB) and VGSCs in detail (slightly modified model: 2]. [11] Further, maturation of the structural organization has been published in December, 2014. Here, the proteins, actin, adducin, $\beta I I-$ spectrin, $\beta I V$-spectrin and ankG have been studied. ${ }^{12}$ Combined, these two publications give a first idea of AIS organization and its structural maturation. Although, recently studies on structural AIS organization get numerous $110[12$ 13, questions remain and results are not proved and analyzed in detail. Such simple models (slightly modified model: 22 mask the full complexity of the AIS. For example, diverse results obtained by $\mathrm{N}$ - and $\mathrm{C}$-terminal labeling of $\beta \mathrm{IV}$-spectrin have not been examined in detail, instead non-verified suggestions, such as freely moving C-terminal tail regions and an increase of the protein densities have been made. 12 [13 It has not been considered that these assumptions do not agree with their published model. This addresses the question of sufficiency and correctness of the model. Within this thesis the main focus is on differential structural organization of $\beta I V$-spectrin and ankG isoforms, and VGSC subtypes, $\mathrm{Na}_{\mathrm{v}} 1.2$ and $\mathrm{Na}_{\mathrm{v}} 1.6$, whereby the different roles of protein isoforms and subtypes are demonstrated (Chapter: 5). Together, the electrophysiological characterization 1 , protein density studies as well as structural organization of VGSCs and cytoskeletal proteins allow the establishment of a new more detailed functional and structural model of the AIS throughout neuronal maturation.

In another part of the project, the consequences of a mutation in $\beta I V$-spectrin were studied. The $q v^{3 J}$ mutant displays a small, but perturbing defect in the cytoskeletal protein $\beta I V$-spectrin. $\beta I V-$ spectrin has been suggested as appearing early in maturation and being the main stabilizer of the AIS architecture. Numerous proteins, such as ankyrins and VGSCs have been reported to

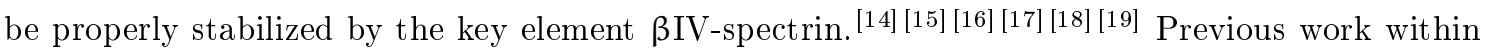
this project revealed similar AP waveforms in the first two weeks of maturation for $q v^{3 J}$ mutant and control neurons. Starting the third week AP onset in $q v^{3 J}$ mutant neurons slowed down significantly. 2 Consequently, the AIS function is affected by the mutation and suggests a defect of VGSC enrichment in the AIS. Most likely are defects of stabilization. Here it is of major interest to monitor the density of VGSCs and the cytoskeletal proteins, $\beta I V$-spectrin and

\footnotetext{
${ }^{2}$ Previous work: Electrophysiology measurements on control (wildtype/heterocygote) and $q v^{3 J}$ mutant neurons were performed by Elinor Lazarov, Hebrew University of Jerusalem, Israel during her PhD thesis. Manuscript: (2) Elinor Lazarov, Melanie Dannemeyer, Michael Gutnick, Fred Wolf, Andreas Neef "Reduced Nav channel density in the axon initial segment slows down the action potential onset and impairs high frequency encoding."
} 
ankG, with maturation (Chapter: 6). Further, a detailed structural study leads towards a better understanding of the mechanism, which impairs information encoding in the $q v^{3 J}$ mutant (Chapter: 7). 2 Thereby, the $q v^{3 J}$ mutant demonstrates the importance of maintaining the AIS structure for its full functionality. 


\section{Introduction}

\subsection{The Neuron}

The hippocampal neuron consists of a cell body, referred to as the soma, and two classes of branches, referred to as axons and dendrites. A typical neuron has one axon and several dendrites, which arise from the soma. Both processes form complex axonal, respectively dendritic trees. The axonal region close to the soma is called the axon hillock. A special about $20 \mu \mathrm{m}$ region of the axon is called the axon initial segment (AIS). 20,21 It contains a special protein composition and arrangement. Upon a stimulus, which leads to a depolarization above the threshold potential, an electrical signal, the action potential (AP), is initiated in the AIS and propagated towards surrounding neurons. The neurons are connected with each other by junctions, referred to as synapses. At these regions the membranes of two neurons are located close to each other and allow the transfer of an electrical or chemical signal from one to another neuron. The signal is received by dendrites of the connecting neurons and propagated towards their soma. A new all or nothing AP is initiated in the AIS of these neurons. 22

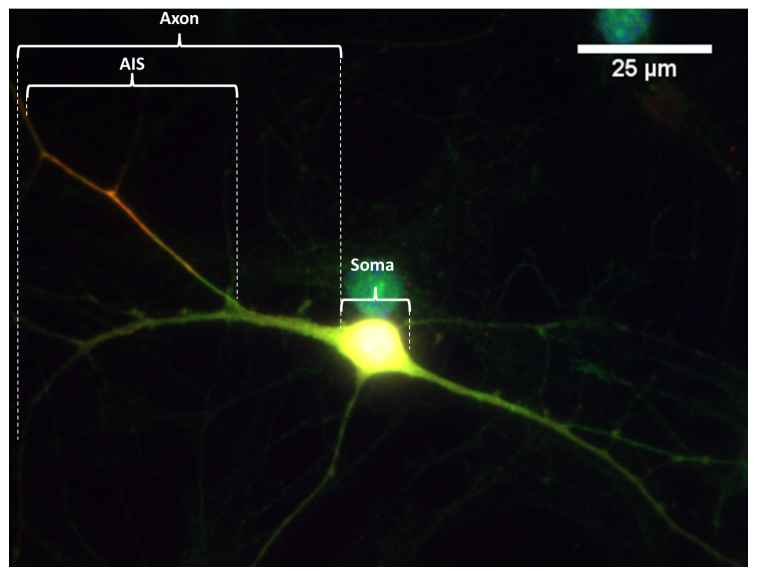

Figure 1: The hippocampal neuron. The image shows a hippocamal neuron at DIV 14, filled with Alexa 568 (yellow) using a patch pipette, stained for voltage-gated sodium channels (VGSCs) with Alexa 488 (red), ankG with Alexa 647 (green) and nucleus with DAPI (blue). Imaging was performed by wide-field microscopy with a $60 \mathrm{x}$ objective (method: 3.4 using an additional excitation (Semrock, BLP01-594R-25) filter to separate the emission light). The main parts of the axon, the AIS and the soma are labeled. Several dendrites arise from the soma. The neuron was patched and filled by Elinor Lazarov (Hebrew University Jerusalem, Israel), labeling and imaging was performed by myself. 


\subsubsection{The Axon Initial Segment}

The axon initial segment (AIS) is a highly specialized neuronal subregion. 21 [5 $[6$ it is located in the proximal region of the axon, directly distal of the axon hillock, where the axon arises from the cell body. ${ }^{6}$ Its exact position varies across and within different cell types and is amongst others modulated by activity. ${ }^{23}$ In recent experiments two days depolarization of mature hippocampal neurons resulted in a significant shift of their AIS. The AIS has been moved up to $17 \mu \mathrm{m}$ further away from the soma. Returning to normal conditions showed reversibility of this process. 24

The length and size of the AIS varies between neuronal cell types and organisms. Early electron microscopy studies (from 1976) of the AIS on purkinje cells (PCs) of rats revealed a length of about $17.1+/-2.0 \mu \mathrm{m}$, a diameter of $0.79+/-0.10 \mu \mathrm{m}$ at the origin, a diameter of $0.73+/-0.19 \mu \mathrm{m}$ at the beginning of myelin and a total surface of about $45.6 \mu \mathrm{m}^{2} .20$

The AIS is involved in action potential (AP) initiation, modulation and propagation by converting the dendritic input into an all or nothing AP. 4 [5 It is highly enriched with ion channels, including voltage-gated sodium channels (VGSCs) ${ }^{[8}$, which lower the threshold for AP initiation and lead to a fast inward current during the rising phase of depolarization. 6 ]

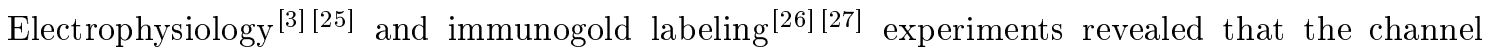
density in the AIS is about 40 to 50 (electrophysiology), respectively 35 to 80 (immunogold labeling) times higher than that of the soma or dendrites. 21 The threshold for initiation depends amongst others on the position of the AIS. More distal located AISs have been shown to have higher thresholds for initiation. 24

Early electrophysiological 25 and immunohistochemistry studies indicated an evenly distribution of VGSCs throughout the AIS. 28$]$ [29] Recent immunohistochemistry experiments combined with novel imaging techniques indicate that the AP is formed at the distal part of the AIS. $30 \sqrt[31]{61}$ The subtype $\mathrm{Na}_{\mathrm{v}} 1.2$ has been identified as being located in the proximal part of the AIS and displays a high threshold, whereas the subtype $\mathrm{Na}_{\mathrm{v}} 1.6$ has been found more distal and displays a low threshold for initiation. 31,6

Besides VGSCs $[7][6]$, the AIS is enriched with associated proteins, such as the adapter protein ankG 32 (details: 2.3), the cytoskeletal linker protein $\beta I V$-spectrin (details: 2.2) and cell adhesion molecules. 33] 34] The composition functions as a barrier between the axonal and somatodendritic compartments for maintenance of neuronal polarity. $35 \sqrt[36]{25}$ The lateral mobility of membrane proteins has been demonstrated to differ in the AIS and distal axon. 36 Some membrane proteins, such as the L1 family proteins, neurofilament (NF) and neuronal adhesion molecule (NrCAM), show a very slow or no diffusion in the AIS. $\frac{35}{35}$ A disruption of the underlying cytoskeleton results in a loss of the barrier and thus an increase of membrane protein diffusion. Highly debated remains the sequence of protein recruitment to the AIS. 
1. $\mathrm{NF}$ and $\mathrm{NrCAM}$ have been proposed to cluster prior ankG in the AIS ${ }^{34}$,

2. but others suggest that ankG first localizes in the AIS and all other proteins ${ }^{\text {[5 }}$, including NF and $\mathrm{NrCAM}$, are recruited by ankG through their ankG binding sites. 6

3. Also, the ankG- $\beta I V$-spectrin complex has been described to form the AIS before ion channels cluster. $37 \lcm{17] 6}$

4. Even the theory that the sodium channel $\beta$-subunit of VGSCs plays a role in recruiting ankG has been suggested. 38

The structural organization of proteins in the AIS is particular.11 112 Actin forms ring-like structures along the entire axon, but not in dendrites. The actin rings are evenly spaced in about $180-190 \mathrm{~nm}$. Adducin ${ }^{[39}$, an actin-capping protein, colocalizes with the actin ring-like structures and composes a ring-like organization. The distance between each actin-adducin ring is about $182+/-16 \mathrm{~nm}$. 111 [12 This corresponds to the length of a spectrin tetramer, which is formed from two $100 \mathrm{~nm}$ long spectrin heterodimers 40 10] 12 , and connects the actinadducin rings with each other by binding to a specific site on the actin (more information: 2.2. Similar organization for actin and spectrin has been described earlier for the cytoskeleton of

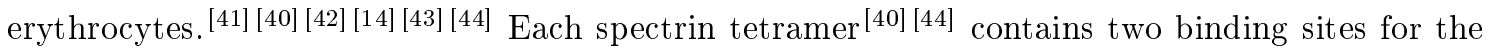
adapter protein ankG. 42 [45] The binding sites are located on the $\beta$-spectrin chains more closely towards the C-termini (more information: 2.2). Due to its two binding sites to spectrin, which are closely located to each other, ankG displays a periodic organization approximately halfway between the actin rings or halfway between the spectrins N-termini (more information: 2.2). 112 VGSCs are highly enriched in the AIS. They are connected to spectrin by binding to the adapter protein ankG. [46] [47] [12] Based on the underlying cytoskeletal structure they appear as an organized pattern halfway between the actin rings (or halfway between the spectrins N-termini, colocalized with ankG). 11112 


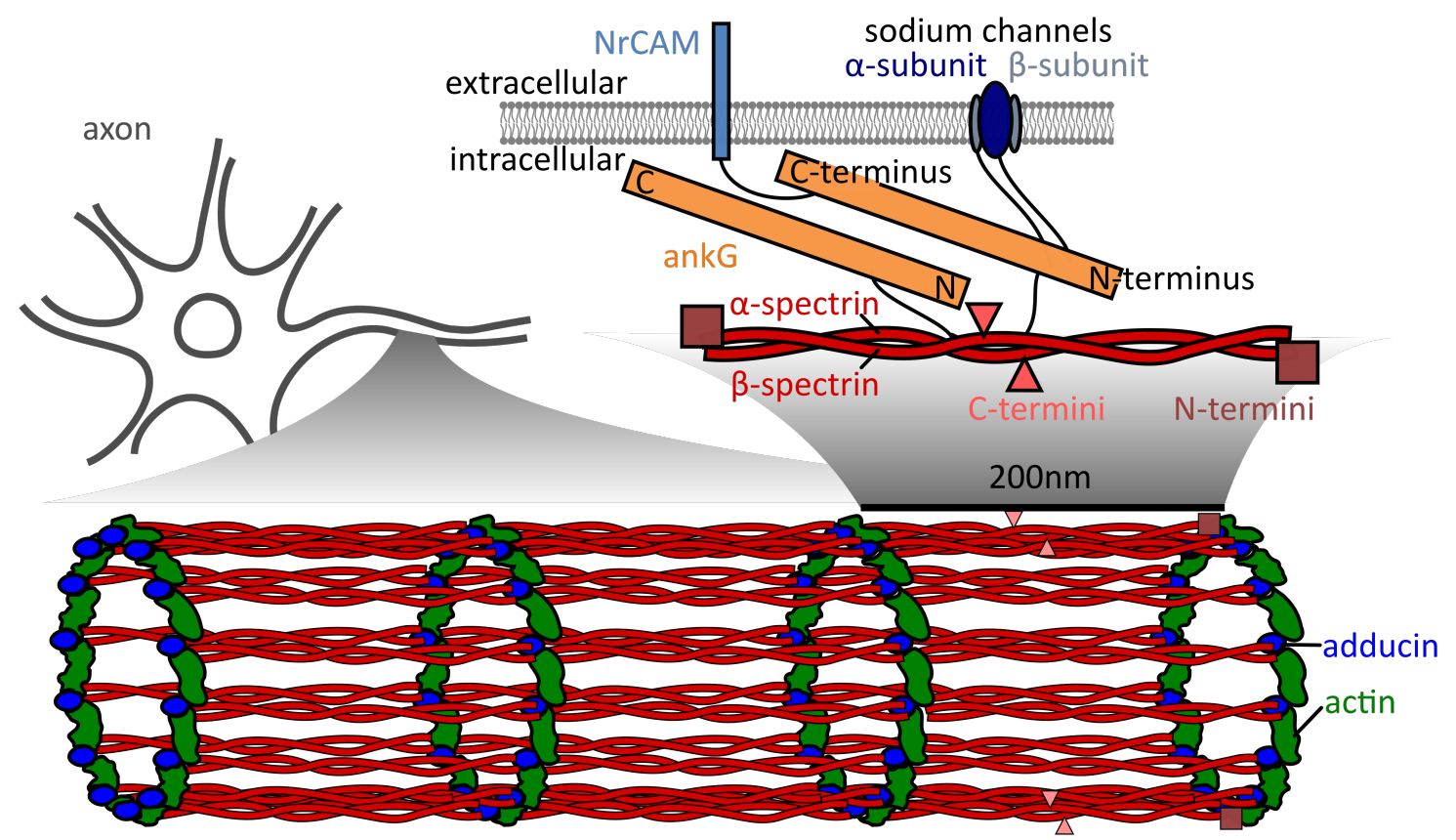

Figure 2: A schematic view of the AIS. Actin, capped with adducin, is organized in ring-like structures. Actin rings are connected by spectrin tetramers with a length of about $200 \mathrm{~nm}$. N-termini of the spectrin tetramers are orientated towards the actin. AnkG binds to ankyrin-binding sites of $\beta$-spectrins. Integral membrane proteins, such as sodium channels and the cell adhesion molecule NrCAM, are connected by ankG to the underlying cytoskeleton. Figure has been modified and extended from [11 [12] [48 49] [25] [6.

\subsection{The cytoskeletal protein spectrin}

Spectrin is a major component of the neuronal membrane skeleton. ${ }^{19}$ It has been first described in red blood cells, erythrocytes [50] [41] [43], where it is part of forming a network amongst others

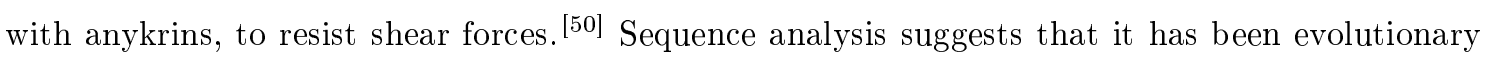
evolved before ankyrins. ${ }^{51][19]}$ Spectrins are distinguished in $\alpha$ - and $\beta$-spectrins. The two major isoforms of $\alpha$-spectrin are encoded by the genes SPTA1 and SPTBAN1. 19 The five $\beta$-spectrins, ßI-V spectrin, are encoded by the genes SPTB, SPTBN1, SPTBN2, SPTBN4, and SPTBN5. 48 The core element of spectrin is a helical repeating unit, the spectrin repeat. ${ }^{[19]} \alpha$-spectrins have typically 20 to 21 and $\beta$-spectrins 17 repeats. 44 Th2 Their sequence homology is less than $30 \%$. 19 Spectrins form tetramers consisting of two $\alpha$ - and $\beta$-spectrins 40$]$ [44] [53] [16], $200 \mathrm{~nm}$ in length. 40] [43] Interactions between two repeats, located close to the C-terminus of $\alpha$-spectrin, repeat 20 and 21 , and two repeats, located close to the N-terminus of $\beta$-spectrin, repeat 1 and $2^{54}$, form a dimer of $\alpha$-and $\beta$-spectrin. ${ }^{48}$ The $\alpha$-spectrin repeat 1 and $\beta$-spectrin repeat 17 of the heterodimer interact with the corresponding repeat of a second heterodimer. $5 5 \longdiv { 5 6 } [ 5 7 \sqrt [ 1 9 ] { [ 5 } \text { This head-to-head association }$ forms a spectrin tetramer. 58$][59]$

In spectrins, a number of domains and binding sites have been identified. An actin-binding domain 
(ABD) $43[60$ mediates the binding of spectrin to the underlying actin network (figure: 2]. [16] [19 Further, a SH3 domain and a PH-domain are found. ${ }^{16} \mathrm{SH} 3-$ and $\mathrm{PH}$-domains are common features of proteins involved in cellular signaling. 19 The role of spectrin e.g. in lymphocyte activation has been described earlier. Additionally, PH-domains play a role in cytoskeletal organization. [19] EF-hand domains, located on the $\alpha$-spectrin, have been shown to modulate the actin-binding domain. ${ }^{[19}$ The ankyrin-binding site is located between the 14 th and 15 th repeat, close to the C-terminus, of each $\beta$-spectrin. [62] [53] [63] [57] [19]

$\beta I V$-spectrin is located on the human and mouse chromosome $19 \mathrm{p} 13.13$ and $7 \mathrm{~b} 2.16$ It is 8789 bp long and has 2559 amino acids. Alternative splicing generates six isoforms of $\beta I V$-spectrin referred to as $\beta \operatorname{IV} \sum 1-\beta \operatorname{IV} \sum 6\left(\beta \operatorname{IV} \sum 1\right.$ Genbank No. NM_020971, $\beta \operatorname{IV} \sum 6$ Genbank No. NM_001199234.1, NM_001199235.1 and NM_001199236.1). [16]17] The role of $\beta I V$-spectrin has been extensively studied. 16 Immunohistochemistry studies showed that $\beta I V$-spectrin colocalizes with ankG, VGSCs and cell adhesion molecules (such as NF and NrCAM) in the AIS. [60 [16 [19 It is commonly accepted that spectrin is recruited by ankG and forms a stable complex with membrane proteins. ${ }^{64}$ Knockout of $\beta I V$-spectrin in mice showed abnormal ankG and VGSC distribution or loss and a quivering phenotype. $[17]\left[18\right.$ Mice lacking only the isoform $\beta I V \sum 1$ showed a quivering phenotype, too. 18 Therefore, $\beta \operatorname{IV} \sum 1$ has been described as being the main isoform of $\beta I V$-spectrin for the stabilization of the AIS and nodes of Ranvier (noR) membranes. $15 \square[16$

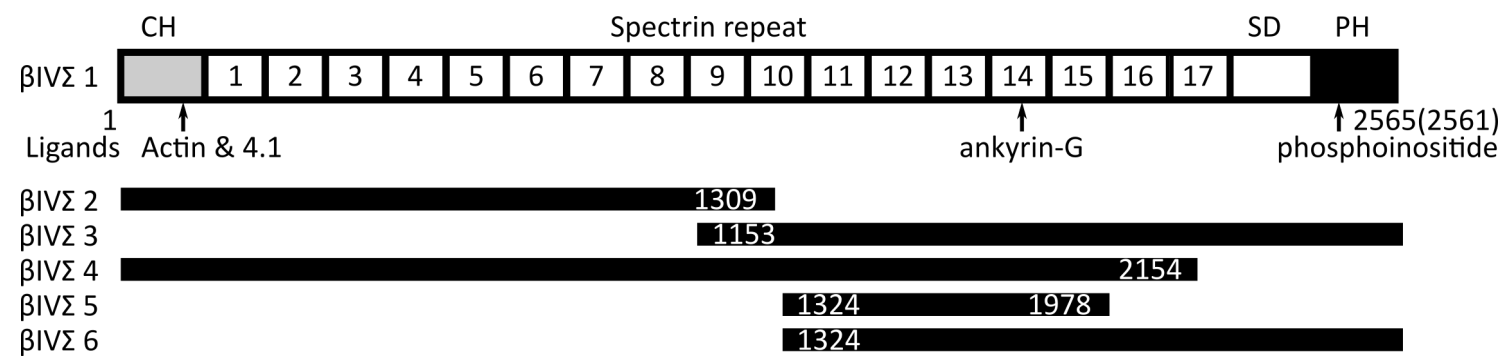

Figure 3: A schematic view of the $\beta I V$-spectrin isoforms. $\beta I V \sum 1$ is shown in detail. $\beta I V \sum 2-\beta I V \sum 6$ aligned to $\beta I V \sum 1$. Amino acid sequences of isoforms do not correspond completely. Short insertions, deletions and extensions of $\beta \operatorname{IV} \sum 2-\beta \operatorname{IV} \sum 6$ relative to $\beta \operatorname{IV} \sum 1$ are ignored. White numbers indicate the relative starting and ending amino acid of each isoform in relation to $\beta \operatorname{IV} \sum 1$. Ligand binding sites are marked. CH, calponin homology domain; SD, specific domain; PH, pleckstrin homology domain, modified and extended [18 Uniprot.org: Q9H254, Q8VBX2.

\subsection{The adapter protein ankyrin}

Ankyrins are a family of large adapter proteins that link integral membrane proteins to the underlying spectrin-actin cytoskeleton. [65] [60] [66] [68] $[69]$ The genes $A N K 1^{[32]}$, ANK2 and $A N K 3^{70}$ code for the three subtypes ankR ${ }^{71}$, ankB and ankG. ${ }^{70}$ Each of them appears as a 
number of specific isoforms resulting from alternative splicing. AnkR has been first detected in red blood cells, erythrocytes, where it links spectrin to the plasma membrane and provides resistance to shear forces. 50 Ankyrins are composed of four functional domains. An N-terminal domain with 24 tandem ankyrin repeats identified as the membrane-binding domain, a spectrin-binding domain (ZU5 1 domain) 45 , a domain that bind proteins of the apoptosis pathway, and a variable Cterminal domain. ${ }^{67}$ A mutation in the spectrin-binding domain (ZU5 1 domain) in giant $480 \mathrm{kDa}$ ankG did not affect its ability to recruit $\beta$-spectrin. Suggesting that the spectrin-binding domain plays no role in spectrin recruitment. Instead, S2417, located in the 1,900 amino acid region, has been identifed to play a role in $\beta$-spectrin binding and recruitment. 73 .

The membrane binding domain of ankyrin is composed of four subdomains, each containing six ankyrin repeats. ${ }^{74}$ The scaffold protein ankG plays a key role in neuronal development, polarity

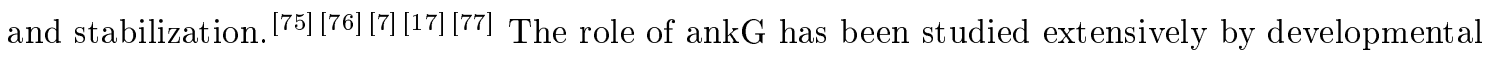
immunohistochemistry in tissues and cell cultures derived from rats and mice including nullmutants, region-specific ankG knockouts and the reduction of the expression by short hairpin

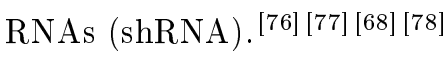

AnkG is highly concentrated in the AIS 32$]$ where it colocalizes with $\beta I V$-spectrin [16] (more information: 2.2 and integral membrane proteins, such as VGSCs $79 \square 0$, NF-186 and NrCAM. $8 1 \longdiv { 7 5 }$ Further, it can be found at a lower level in the cell bodies of neurons. 82,83 It is expressed at stage $\mathrm{P} 2^{[7}$ and therefore one of the earliest expressed proteins. ${ }^{7}[\sqrt[5]{5}$ The unique serine-rich domains and the highly divergent tail domain present in the isoforms 480and $270-\mathrm{kD}$ ankG $[32]$ contribute to its highly specific restriction to the AIS and limit its lateral diffusion. 82$] 84$

The lack of ankG results in a complete loss of the cytoskeletal protein $\beta I V$-spectrin, which is linked by ankG to the plasma membrane. $\frac{76}{17}$ Opposite, $\beta I V$-spectrin links the ankG-membrane protein complex to the underlying cytoskeleton. Concluding, ankG is necessary for the assembly of the spectrin-based cytoskeleton. [65 [76] In ankG knockout mice abnormal distribution or complete loss of the $\mathrm{NrCAM}[7$ and VGSC have been observed. Controversial, NF has been described as appearing normal, disrupted ${ }^{[7}$ or completely lost ${ }^{[2]}$. Knockdown of NF or NrCAM does not prevent the formation of an ankG based AIS in cultured hippocampal neurons. 67 [34 Deletion of the sodium channel $\alpha$-subunit had no effect, but deletion of the $\beta$-subunit had an effect on recruiting ankG to the AIS. $\frac{38}{38}$ However, the study could not rule out that the $\beta$-subunit plays an indirect, but not direct role in recruiting ankG. ${ }^{38}$ Therefore it is commonly accepted in the field

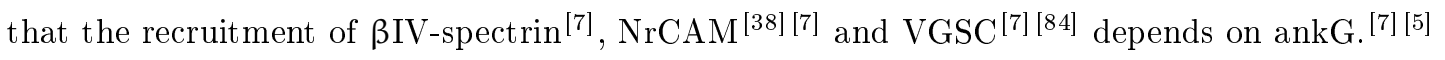
Further, silencing of ankG expression caused the axon to show dendritic characteristics. 85 Proteins, normally restricted to the somatodendritic domains, such as MAP2, have been detected 
in the axon. 85 Proteolysis of the ankG- $\beta$ IV-spectrin complex resulted in a loss of VGSCs. $\frac{86}{86}$ Both results support that ankG has a role in the maintenance of neuronal polarity. ${ }^{85}$ [86] The role of ankG in VGSC stabilization $\frac{76}{78}$ has been supported by site-directed mutants with decreased affinity for ankG and increased diffusion speed of VGSCs. ${ }^{84}$ Channels not anchored by ankG are removed by endocytosis. 87

In many integral proteins cytoplasmic ankG binding sites have been identified. This includes $\mathrm{NF}, \mathrm{NrCAM}$ [86], VGSCs and potassium channels. NF and NrCAM bind through a conserved domain (FIGQY motif) in their cytoplasmic C-termini to ankG. 88 The channel subtypes $\mathrm{Na}_{\mathrm{v}} 1.2$ and $\mathrm{Na}_{\mathrm{v}} 1.6$ show a common ankG binding domain in the cytoplasmic II-III loop of the $\alpha$ subunit. $7 7 \longdiv { 8 9 } [ 9 0 \text { Region-specific mutations of the ankG binding domain of } \beta I V \text { -spectrin and other }$ AIS proteins have shown that it is crucial for their AIS localization. 17

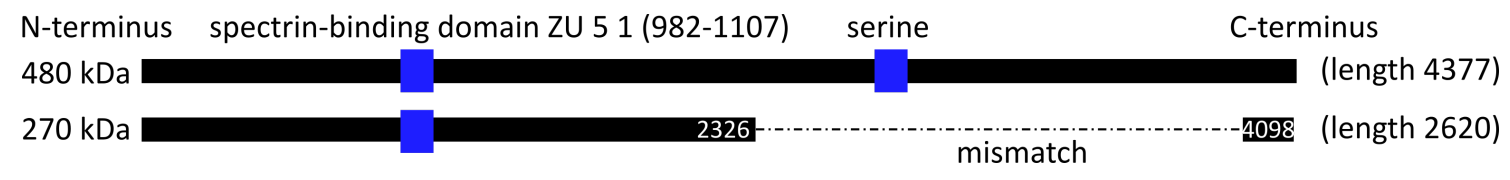

Figure 4: A schematic view of the giant $480 \mathrm{kDa}$ and the $270 \mathrm{kDa}$ ankG isoforms. Giant $480 \mathrm{kDa}$ ankG is shown in full length. $270 \mathrm{kDa}$ ankG has been aligned. Amino acid sequences do not correspond completely. Short insertions, deletions and extensions of $270 \mathrm{kDa}$ ankG are ignored. White numbers indicate the relative starting and ending amino acid of $270 \mathrm{kDa}$ with respect to giant $480 \mathrm{kDa}$ ankG. The spectrin-binding domain and the S2417, located in the 1,900 amino acid region, are marked (blue). Established after [73 and Uniprot.org: Q12955, W6PPA1.

\subsection{Voltage-gated sodium channels}

Channels are pore-forming units within the membrane of cells. Sodium channels mediate the selective influx of sodium ions from the extracellular region through the membrane into the cell. It is distinguished between ungated and gated sodium channels. Whereas ungated sodium channels are always open, underlie gated sodium channels a regulartory mechanism. Gated sodium channels are particular found in neurons. The gating mechanism can be conrolled either by chemical or electrical signals. In voltage-gated sodium channels (VGSCs) gating depends on an electrical signal. 22

VGSCs $\left(\mathrm{Na}_{\mathrm{v}}\right)$ consist of $\alpha$ - and $\beta$-subunits. 91 The pore-forming $\alpha$-subunit has four homologous protein domains (I-IV) ${ }^{92}$, each of them with six transmembrane segments. The $\beta$-subunits have a single transmembrane segment with an extracellular N-terminus and a cytoplasmic C-terminus. $\beta$ subunits modulate $\alpha$-subunits and regulate channel gating. 93,92 Ten genes have been identified to encode the $\alpha$-subunit, referred to as $\mathrm{Na}_{\mathrm{v}} 1.1-\mathrm{Na}_{\mathrm{v}} 1.9$ (Genes: SCN1A - SCN5A, SCN8A, SCN9A - SCN11A) and $\mathrm{Na}_{\mathrm{v}} \mathrm{x}(S C N 7 A) .94$ Four genes encode the $\beta$-subunits $\mathrm{Na}_{\mathrm{v}} \beta 1-\mathrm{Na}_{\mathrm{v}} \beta 4$ (Genes: 
$S C N 1 B-S C N 4 B)$, whereas $\mathrm{Na}_{\mathrm{v}} \beta 1$ assembles with the $\alpha$-subunits $\mathrm{Na}_{\mathrm{v}} 1.1$ to $\mathrm{Na}_{\mathrm{v}} 1.7, \mathrm{Na}_{\mathrm{v}} \beta 2$ with $\mathrm{Na}_{\mathrm{v}} 1.1, \mathrm{Na}_{\mathrm{v}} 1.2, \mathrm{Na}_{\mathrm{v}} 1.5$ to $\mathrm{Na}_{\mathrm{v}} 1.7, \mathrm{Na}_{\mathrm{v}} \beta 3$ with $\mathrm{Na}_{\mathrm{v}} 1.1$ to $\mathrm{Na}_{\mathrm{v}} 1.3, \mathrm{Na}_{\mathrm{v}} 1.5$ and $\mathrm{Na}_{\mathrm{v}} \beta 4$ with $\mathrm{Na}_{\mathrm{v}} 1.1$ to $\mathrm{Na}_{\mathrm{v}} 1.2$ and $\mathrm{Na}_{\mathrm{v}}$ 1.5. 95

The channel subtypes $\mathrm{Na}_{\mathrm{v}} 1.7, \mathrm{Na}_{\mathrm{v}} 1.8$ and $\mathrm{Na}_{\mathrm{v}} 1.9$ are highly concentrated in the pheriperal nervous system (PNS). ${ }^{69} \mathrm{Na}_{\mathrm{v}} 1.4$ and $\mathrm{Na}_{\mathrm{v}} 1.6$ are found in cardiac and skeletal muscles. The subtypes $\mathrm{Na}_{\mathrm{v}} 1.1^{[29}, \mathrm{Na}_{\mathrm{v}} 1.2^{29]}, \mathrm{Na}_{\mathrm{v}} 1.3$ and $\mathrm{Na}_{\mathrm{v}} 1.6^{[97 \sqrt[29]{82}}$ are mainly expressed in the adult central nervous system (CNS). 69 [27] $\mathrm{Na}_{\mathrm{v}} 1.2$ and $\mathrm{Na}_{\mathrm{v}} 1.6$ are the main subtypes of the AIS [29] [31], whereas $\mathrm{Na}_{\mathrm{v}} 1.1$ and $\mathrm{Na}_{\mathrm{v}} 1.3$ are somatodendritic located. 69

Immunohistochemistry experiments and modeling studies found differentiated expression of the channel subtypes $\mathrm{Na}_{\mathrm{v}} 1.2$ and $\mathrm{Na}_{\mathrm{v}}$ 1.6. 31 The dominant channel subtype expressed in the AIS is $\mathrm{Na}_{\mathrm{v}}$ 1.6. It has been shown to have a proximal to distal gradient, meaning its concentration increases with increase of the distance from the soma. Analysis of $100.0 \mu \mathrm{m}$ long immunostainings of the axon showed that the anti- $\mathrm{Na}_{\mathrm{v}} 1.6$ staining intensity peaks at a distance of 30 to $50 \mu \mathrm{m}$ from the soma. 31 Na $\mathrm{Na}_{\mathrm{v}} 1.6$ has not been detected in somatodendritic parts, indicating that it is expressed at a very low concentration or even exclusively confined to the AIS. 26 The distal located $\mathrm{Na}_{\mathrm{v}} 1.6$ channels set a low threshold for AP initiation and have been found to characterize the distal site for AP initiation. 20

$\mathrm{Na}_{\mathrm{v}} 1.2$ channels have been found to be localized proximal of the axon. Immunohistochemistry with anti-panNav showed an intensity peak at 10 to $20 \mu \mathrm{m}{ }^{[31}(20 \text { to } 40 \mu \mathrm{m})^{64}$ from the soma. 10 to $20 \mu \mathrm{m}$ corresponds to the proximal located $\mathrm{Na}_{\mathrm{v}} 1.2$ channels. 31 The proximal $\mathrm{Na}_{\mathrm{v}} 1.2$ channels have a high threshold and have been found to be responsible for backpropagation. Removel of $\mathrm{Na}_{\mathrm{v}} 1.2$, but not $\mathrm{Na}_{\mathrm{v}} 1.6$, results in failure of AP backpropagation. 31

In noR a developmental switch between $\mathrm{Na}_{\mathrm{v}} 1.2$ and $\mathrm{Na}_{\mathrm{v}} 1.6$ has been observed. In early development $\mathrm{Na}_{\mathrm{v}} 1.2$ is strongly expressed and later replaced by $\mathrm{Na}_{\mathrm{v}} 1.6 .98$ At the noR of adults $\mathrm{Na}_{\mathrm{v}} 1.2$ is not detectable in more than $80 \%$ of the neurons. ${ }^{99}$ In the AIS no developmental switch has been observed. $\mathrm{Na}_{\mathrm{v}} 1.2$ is the main subtype in early stages, but stays throughout maturation as $\mathrm{Na}_{\mathrm{v}} 1.6$ increases. At P9 $\mathrm{Na}_{\mathrm{v}} 1.6$ expression is comparable to adults. The increase of $\mathrm{Na}_{\mathrm{v}} 1.6$ expression at noR and AIS correlates. $[8$

As mentioned above, (in 2.3 both the channel targeting and clustering in the AIS depend on ankG 89 The sodium channel $\alpha$-subunit contains a nine amino acid ankG-binding motif $((\mathrm{V} / \mathrm{A}) \mathrm{P}(\mathrm{I} / \mathrm{L}) \mathrm{AXXE}(\mathrm{S} / \mathrm{D}) \mathrm{D})$ between its intracellular loops II and III linking them to the

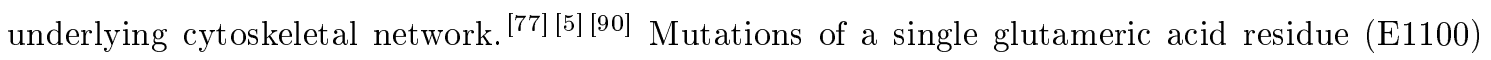
within the motif blocked $\mathrm{Na}_{\mathrm{v}} 1.6$ targeting in vitro and in vivo, indicating that the motif is essential and sufficient for ankG binding. 90

Sodium channel $\beta$-subunits interact through an extracellular domain with L1 family adhesion 
proteins, such as NF-186 and NrCAM, playing a role in their localization. 138

Both, alterations and the loss of $\mathrm{Na}_{\mathrm{v}} 1.2$ and $\mathrm{Na}_{\mathrm{v}} 1.6$ cause major diseases, such as epilepsy and motor dysfunctions, leading to early death. 4101 102 9 $\mathrm{Na}_{\mathrm{v}} 1.6$ null mice die before four weeks of age. 103

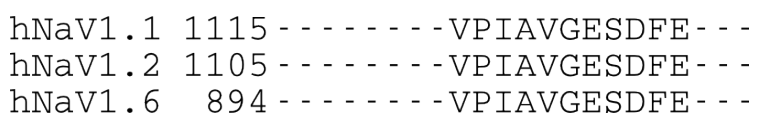

Figure 5: Conserved sequence of ankG binding motifs in sodium channel subtypes, modified from 68 . Sodium channels share the same common motif.

\subsection{The quivering mutation}

The autosomal recessive quivering $(q v)$ mutations arose spontaneous in 1953. Six mutations have been described. Phenotypic expressions are progressive paralysis, ataxia, deafness and tremor starting days after birth. Tail stiffening starting about 30 days after birth, eye infections and sterility of males have been described. Males have found to produce viable sperm, but sired no litters. Females have found to nurse their litters. $Q v$ die before 5 month of age. ${ }^{104}$ Early studies found mouse homocygote to $q v / q v$ being unresponsive to sound, although no abnormalities of their cochleas (auditory portion of the inner ear) have been found. In contrast responses from brainstem auditory nuclei showed abnormalities, indicating a central origin. $1 0 5 \longdiv { 1 0 6 } Q v ^ { 3 J }$ is a loss of function mutation in the $\beta I V$-spectrin 4 gene (Spnb4). A single inserted base (InsT6786) resulted in a frameshift at amino acid G2209 and a new 49-amino-acid extension. This extension does not show homologies with the wildtype. The complete mutant peptide is about $14 \%$ shorter than the wildtype peptide. 1 Immunohistochemistry experiments showed a loss of $\beta$ IV-spectrin associated with ion alterations. $\frac{107}{107}$ The AIS of young 1.5 month old had a higher sodium channel density than the AIS of 6 month old $q v^{3 J} / q v^{3 J} .64$ It has been suggested that the function of the spectrin tetramer to anchor and stabilize channels is defective. No abnormalities for targeting and clustering of ankG have been found. 64 


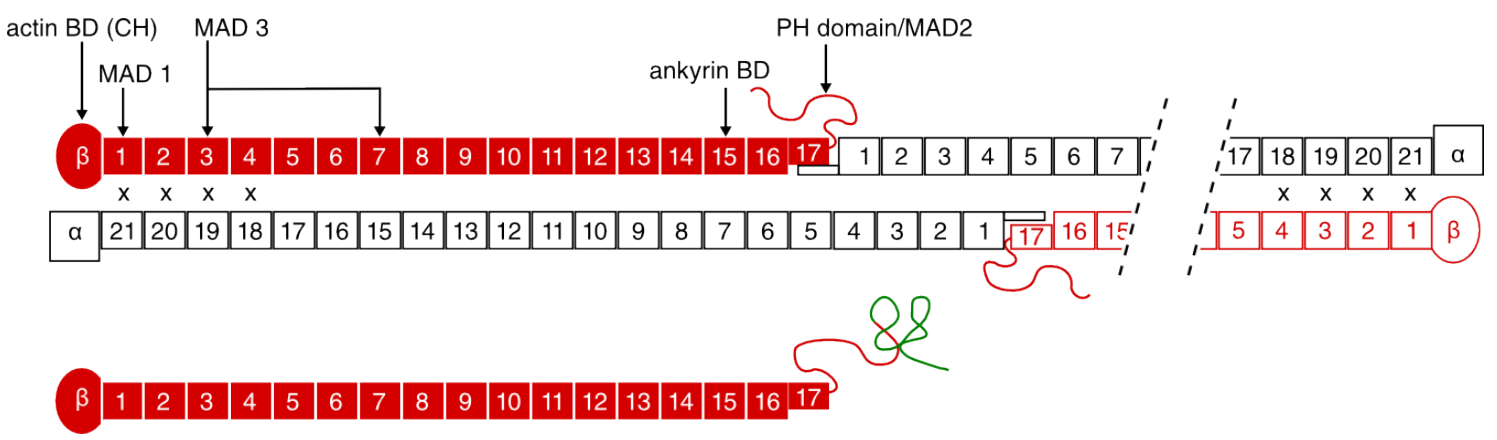

Figure 6: (Top) The spectrin tetramer. $\beta$-subunits with 17-spectrin repeats (shown in red, numbered 1-17). $\alpha$-subunits with 21-spectrin repeats (shown in white, numbered 1-21). Interaction domains are mapped to the $\beta$ spectrin peptide. At the N-terminal region, the actin-binding domain (ABD) consisting of two calponin homology $(\mathrm{CH})$ domains. At the C-terminal region the pleckstrin homology $(\mathrm{PH})$, membrane-association (MAD2) and $\beta I V-$ spectrin specific 'ERQES' domains. The membrane-association domain MAD1 in repeat 1 and MAD3 domains in repeats 3 and 7. The ankyrin-binding domain (ankyrin BD) located in repeat 15 . Crosses represent the region of $\alpha$ and $\beta$-peptides for heterodimer association. (Bottom) The predicted $q v^{3 J}$ mutant peptide. A new 49-amino-acid extension in $\beta I V$-spectrin (green), modified from 1 .

\subsection{Imaging}

STORM, stochastic optical reconstruction microscopy ${ }^{108}$, belongs to the super-resolution imaging methods. This term summarizes imaging methods, which overcome the diffraction limit (about

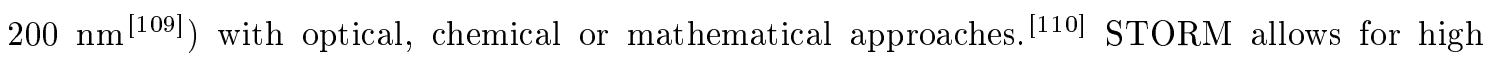
resolution by temporal separation of unresolvable structures. 110 111 The lateral resolution has been reported with about $20 \mathrm{~nm}$.110] [111] Its main advantage is that no high-cost equipment is required. Furthermore, it can be combined with a high number of different fluorescent probes, such as photoactivable fluorescent proteins and organic dyes. 112 STORM has been excessively applied to study biological probes. $1 1 0 \longdiv { 1 1 3 } \lcm { 1 1 } \lcm { 1 2 }$

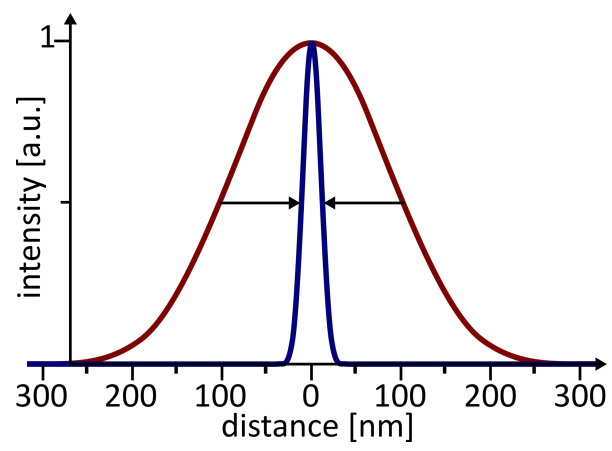

Figure 7: Schematic resolution of wide-field (red) versus STORM (blue) microscopy. Intensity profile of one emitter shows the PSF function of the microscopes [nm].

The target molecule or structure is labeled by a fluorescent probe having a fluorescent ON and 
a non-fluorescent OFF state. 108 STORM relies on the sequential activation and inactivation of individual fluorophores. The majority of fluorophores is transferred into the OFF state. ${ }^{111}$ A sparse resolvable subset of fluorophores is activated, transferred into the ON state, their positions are determined and transferred back into their OFF state. 111] OFF states have been observed in the range of $10.0 \mathrm{~ms}$ to several seconds. 111 At the next point in time, a different subset of fluorophores is activated and their positions are determined. 108 The image is reconstructed from all determined positions. 108] [111] In order to reconstruct a full image at least 2,000 frames need to be recorded. 111 To ensure a full structural reconstruction of a protein of interest frame numbers between 4,000 and 10,000 are recommended. The exact frame number depends on the probe, especially its label density and structural complexity. 111

The simplest mode is one-color STORM with continuous illumination. Thereby, a single laser wavelength e.g. $647 \mathrm{~nm}$ is used to transfer the fluorophores to the OFF state, activate a sparse subset of dyes to the fluorescent state, exciting fluorescence and switching them OFF, allowing for fast imaging. 114 [115
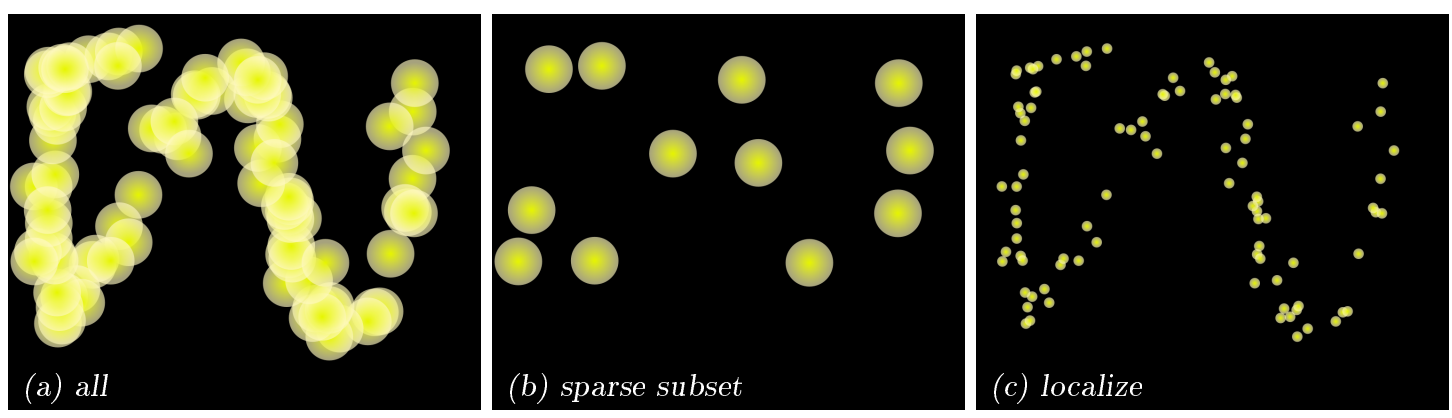

Figure 8: The concept of STORM imaging. (a) The structure of interest is labeled with photoswitchable fluorophores. The fluorophores are in the ON state. All fluorophores are transferred into a non-fluorescent OFF state by irradiation with a laser of suitable wavelength. (b) Spontaneously, a sparse subset is activated. Images are recorded in a specific timescale. (c) Reconstruction of a STORM image by localization of fluorophore positions from each recorded frame. Modified from 111 .

Organic dyes are reversible switchable and can be converted in a fluorescent ON and OFF state multiple times. $108[111[112$ The fluorophores switches between the singlet ground state and the excited state by emitting photons. Upon laser illumination the fluorophore can enter the triplet state by intersystem crossing with the rate $k_{i s c}$. By reacting with molecular oxygen it reenters the singlet ground state. If molecular oxygen is removed by an oxygen scavenger system this process is reduced or even eliminated. By adding a thiol, the fluorophore can form a radical anion $(\dot{\mathrm{F}})$ with the rate $k_{r e d}$ and its reentering of the singlet ground state is highly delayed. By reaction with molecular oxygen it reenters the singlet ground state with the rate $k_{o x}$. 111

ON and OFF states can be highly modulated by the chemical constitution of the applied imaging 
buffers. The OFF state is favored by an increase of the concentration of the thiols. $\frac{115}{15}$ The concentration of reducing thiolates can be either increased by adding a higher concentration or by an increase of the $\mathrm{pH}$ of the imaging buffer. ${ }^{[116}$ The $\mathrm{ON}$ state rate is increased by the application of a $400-405 \mathrm{~nm}$ violet-light laser, which increases the activation rate and reduces the acquisition time. 1111115 The decrease of the ON rate is achieved by removing oxygen with an oxygen scavenger system. Imaging buffers should be adjusted such that $0.1-1.0$ fluorophores per $\mu \mathrm{m}$ on each frame are ON. 1111

In the majority of publications Alexa 647 is recommended to be used with the thiol MEA (cysteamine hydrochloride, $\beta$-mercaptoethylamine), a relative high $\mathrm{pH}$ value of 8.0 - 8.5 and an oxygen scavenger system (e.g. an enzymatic system containing glucose oxidase and catalse). 111] 115 Recommendations for Alexa 488 are more diverse. It has been described to work well adding low concentrations of the thiol MEA, 1 - $10 \mathrm{mM}$, high concentrations of MEA, $50-200 \mathrm{mM}$, or MEA in combination with an oxygen scavenging system. $117[118][111$

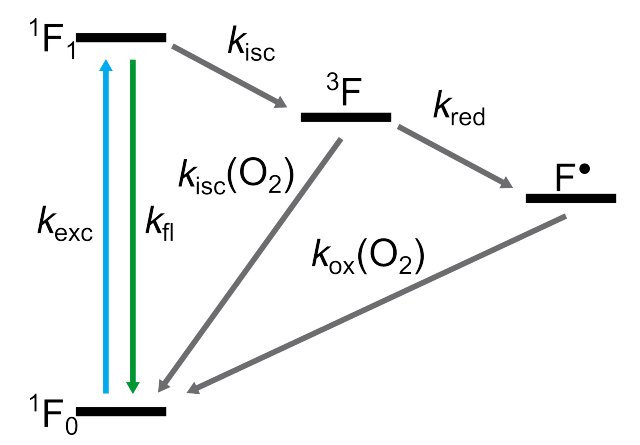

Figure 9: Photoswitching of Alexa dyes. Modified from 111.

The resolution is limited by the fluorophore density according to the Nyquist sampling

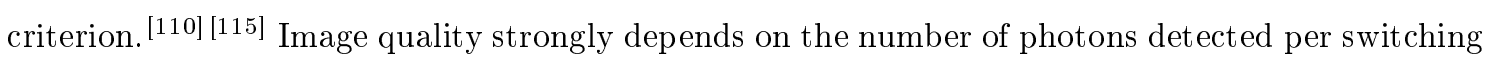
cycle and the ON-OFF duty cycle, the time a fluorophore spends in the ON state. ${ }^{119}$ For example Alexa 647 has a high number of photons per switching cycle and a low ON-OFF duty cycle and is optimal suitable to resolve small structures. $\frac{115}{}$ 


\section{Materials and Methods}

\subsection{Cell culture}

The C57BL/6N mice were maintained and provided by the Tierhaus, Max Planck Institute for Experimental Medicine. $Q v^{3 J}$ mutant mice were provided by Dr. Nicola Strenzke and Gulnara Yamanbaeva, InnerEarLab, The University Medical Center Göttingen. Breeding of $q v^{3 J}$ mutants was performed by the animal caretaker Daniela Marschall, Zentrale Tierexperimentelle Einrichtung (ZTE), The University Medical Center Göttingen.

Protocols for hippocampal neuron dissection and cultivation were provided by the technical assistant Sabine Klöppner, Max-Planck Institute for Experimental Medicine. General assistance with cell culture was received from the technical assistant Sabine Klöppner, Max-Planck Institute for Experimental Medicine.

Preparation of neuronal cultures was performed by myself.

Primary hippocampal cultures were prepared from mice embryos (E17 - 18) (described earlier 120 ) (mouse inbred strain $C 57 B L / 6 N)$ and newborn mice $(\mathrm{P} 0)$ (described earlier ${ }^{[121}$ ) ( $q v^{3 J}$ mutant). Hippocampi were isolated. E18 hippocampi were collected in Neurobasal medium (Life Technologies, 12348-017) and P0 hippocampi were collected in Neurobasal-A medium (Life Technologies, 12349-015) with 100 mM HEPES buffer solution (Life Technologies, 15630-056). Afterward hippocampi were digested with Trypsin/EDTA (Biochrom) 0.05 \%/0.02\% (w/v) in PBS at $37^{\circ} \mathrm{C}$ for 15 min.. Trypsin reaction was stopped with $10 \%$ FCS (Biochrom, Cat. 1221) in medium. The hippocampi were transferred to Neurobasal or Neurobasal-A medium with 1:50 B-27 Supplement (Life Technologies, 17504-044), 1:400 Glutamax (Life Technologies, 35050-038) and $0.1 \mu \mathrm{g} / \mathrm{mL}$ bfgf recombinant human protein (Life Technologies, 13256-029). For bfgf stock solution $10 \mu \mathrm{g}$ powder was diluted in $10 \mathrm{mM}$ TRIS pH 7.6 with $0.1 \%$ BSA. Hippocampi were pipetted up and down, and centrifuged several times until tissue was dissolved.

Wildtype (E17 - 18) derived neurons were seeded on $0.1 \mathrm{mg} / \mathrm{mL}$ poly-L-lysine-coated (SigmaAldrich, P2636) coverslips (Thermo Scientific, Menzel-Glaeser $\varnothing 10 \mathrm{~mm} \# 1$ ) for wide-field imaging. 200,000 to 250,000 neurons per $2 \mathrm{~mL}$ were added in one dish with seven coverslips. For STORM imaging neurons were seeded in polylysine-coated (Sigma-Aldrich, P2636) 4-well chambers (Thermo Scientific nunc, 155383 or Sarstedt, REF94.6190.402); 60,000 to 80,000 neurons per well. Neuron derived from newborn offspring from $q v^{3 J}$ mutant were cultivated separately, one offspring per $2 \mathrm{~mL}$ and seeded either on slides (6) or in chambers (2 wells). Neurons were cultivated at $37^{\circ} \mathrm{C}$ with $8.0 \% \mathrm{CO}_{2}$. Half of the medium was exchanged with freshly prepared medium once a week. 


\subsection{Genotyping}

Genotyping of $q v^{3 J}$ mutants was performed by the technical assistants Sandra Gerke and Christiane Senger-Freitag, InnerEarLab, The University Medical Center Göttingen.

Breeding of $q v^{3 J}$ was performed with heterocygote parent animals and an expected rate of $50.0 \%$ heterocygote, $25.0 \%$ wildtype and $25.0 \%$ mutant offsprings. The obtained offspring were $53.7 \%$ heterocygote, $23.3 \%$ wildtype and $23.0 \%$ mutant and is in agreement with expected ratios.

Genotyping of $q v^{3 J}$ P0 offspring was performed using tale biopsies. Primer pairs were $\mathrm{F}$ (5'AGG CAG CGC CTT TGC TGC GTC-3') and R (5'-TCC TGG TCA CAG AGG TCC TTA-3'). Final PCR contained 1.0 $\mu \mathrm{L}$ DNA, $0.2 \mu \mathrm{L}$ of each primer, $0.4 \mu \mathrm{L}$ DreamTaq DNA Polymerase (Thermo Scientific, EP0703), and PCR buffer (containing Tris-HCl (pH 8.8), ammonium sulphate (Sigma), MgCl2 (Sigma-Aldrich), 2-mercaptoethanol (Merck), EDTA (pH 8.0) (Sigma), nucleoside triphosphates (dATP, dCTP, dGTP, dTTP) (Promega), BSA (Ambion - Life Technolgies) and $\mathrm{H} 2 \mathrm{O})$.

PCR program:

1. Initial denaturation: 3 min., at $94{ }^{\circ} \mathrm{C}$

2. $36 \times$ cycles of

(a) Denaturation: 30 sec., at $94^{\circ} \mathrm{C}$

(b) Annealing: 30 sec., at $60{ }^{\circ} \mathrm{C}$

(c) Elongation: 60 sec., at $74{ }^{\circ} \mathrm{C}$

3. Final elongation: 7 min., at $72{ }^{\circ} \mathrm{C}$

PCR product was proved by $2 \%$ gel electrophoresis. Enzymatic digestion was performed with Sty I (10 U/ $\mu \mathrm{L})$ (New England Biolabs, R0500S). Product was separated by $3 \%$ gel electrophoresis. Figure 10 shows an example of a gel from genotyping the $q v^{3 J}$ offspring. The bands are clearly visible at 250,350 and 600 (wildtype $+/+600 \mathrm{bp}$, heterocygote $+/-600 \mathrm{bp}+350 \mathrm{bp}+250 \mathrm{bp}$, mutant $-/-350 \mathrm{bp}+250 \mathrm{bp}$ ) indicating a high quality of the gel. 


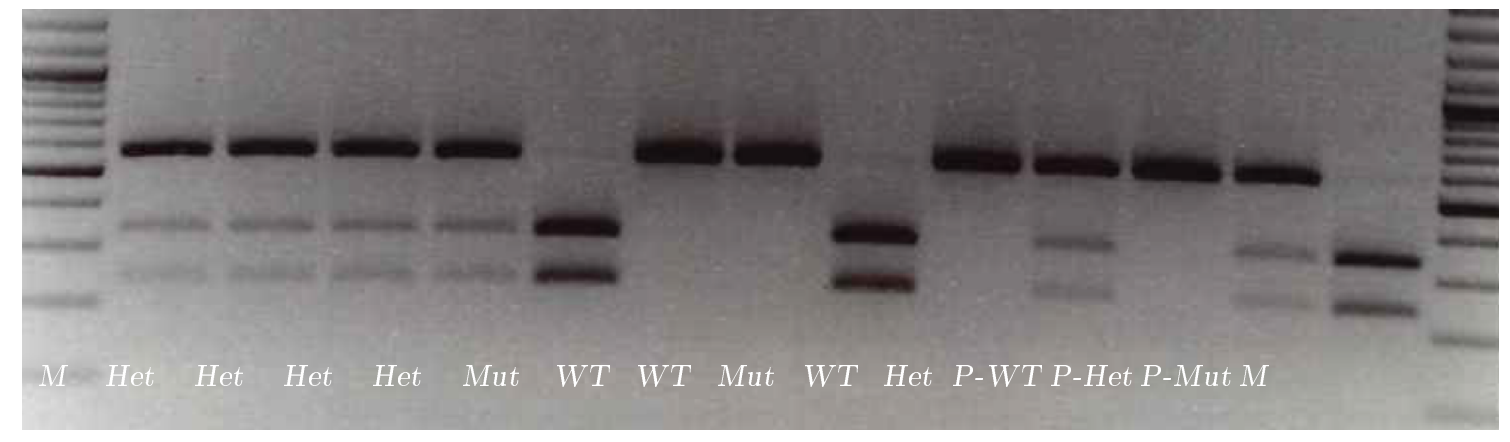

Figure 10: Example of genotyping results from $q v^{3 J}$ mutant. $\mathrm{M}=$ Marker, WT $=$ wildtype, Mut $=$ mutant, Het $=$ heterocygote, $\mathrm{P}-\mathrm{WT}=$ positive control wildtype, P-Mut $=$ positive control mutant, P-Het $=$ positive control heterocygote. Obtained by Sandra Gerke and Christiane Senger-Freitag.

\subsection{Immunohistochemistry}

The immunohistochemistry protocol was provided by Elinor Lazarov, Hebrew University Jerusalem, Israel. Neurons were fixed and stained by myself.

Neurons were fixed at different days in vitro $(D I V s)$ with Microscopy Formaldehyde solution $4 \%$ buffered pH 6.9 (Merck, 100496) at $4{ }^{\circ} \mathrm{C}$ for 8 min. and permeabilized in $0.5 \%$ (v/v) Triton-X-100 (Sigma-Aldrich, X100) at room temperature for $5 \mathrm{~min}$., followed by a $1.5 \mathrm{~h}$ blocking with buffer (3\% (w/v) BSA (Sigma-Aldrich, A0281) in 1x PBS (Life Technologies, 18912-014) and $0.1 \%$ (v/v) Tween20 (Sigma-Aldrich, P7949)). The samples were incubated with primary antibodies (list: 11.2 in blocking buffer at $4{ }^{\circ} \mathrm{C}$ overnight. Afterward they were washed and stained with secondary antibodies (list: 11.2) in blocking buffer at room temperature for 45 min.. Coverslips were mounted with ProLong Gold antifade reagent with DAPI (Molecular Probes, P36935) to glass slides and stored at $4{ }^{\circ} \mathrm{C}$. Cultures in chambers were postfixed with Microscopy Formaldehyde solution $4 \%$ at room temperature for 5 min., washed several times with $1 \mathrm{x}$ PBS and stored in 1x PBS at $4{ }^{\circ} \mathrm{C}$. 


\subsection{Microscopy}

In this thesis two different microscopy methods were applied: Conventional wide-field microscopy and stochastical optical reconstruction microscopy (STORM).

\subsubsection{Wide-field microscopy}

Images were recorded using an inverted wide-field microscope (Olympus IX-71) equipped with a water-immersion objective lens (Olympus, UPlanSApo, 60x magnification, NA 1.2). UV (excitation $394 \mathrm{~nm}$ ), cyan (excitation $476 \mathrm{~nm}$ ), and red (excitation $634 \mathrm{~nm}$ ) LEDs (Lumencor, Spectra X light engine) were used for excitation of DAPI, Alexa 488 and Alexa 647 (exposure time for Alexa 488 and Alexa $6470.1 \mathrm{~s}$; exposure time for DAPI $0.02 \mathrm{~s}$ ), respectively. The fluorescence light was separated from the LED light with a dichroic beam splitter (Chroma Technologies, 59004bsChroma Filters) and additional excitation filters (Semrock, BLP01-405R-25 for DAPI; Semrock, FF01-520/35-25 for Alexa 488; and Semrock, BLP01-635R-25 for Alexa 647) before being imaged on an electron multiplying CCD camera (Andor, EMCCD; DU-897-CS0-BV) with an effective pixel size of $160 \mathrm{~nm}$; temperature of camera $-50{ }^{\circ} \mathrm{C}$.

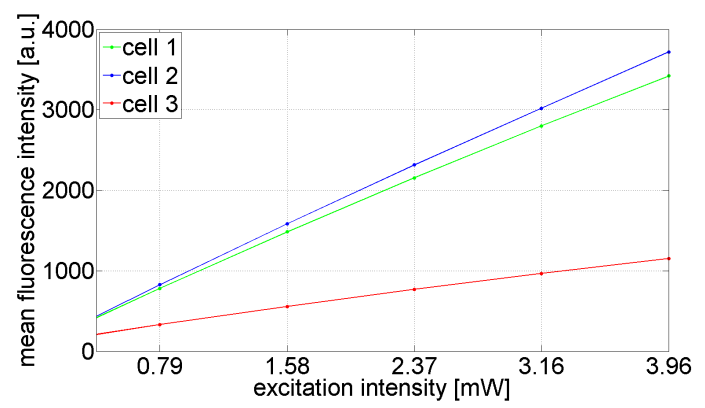

Figure 11: Intensity behavior. Checking for linear intensity behavior of the wide-field (details: 3.4 setup. In some cases, especially high $D I V s$, neurons could not be imaged with a $100 \%$ excitation intensity. In order to ensure that the LED excitation intensity behaves proportional to the emission intensity, calibrations were performed. Three neurons labeled for ankG (C-terminal antibody, sc-28561, 11.2 with Alexa 488 imaged with 2, 4, 10, 20, 30, 40 and $50 \%$ LED intensity. On every image the mean intensity of the same area was determined and plotted. The behavior was approximated as being linear, although in some cases slight deviations were measured. 


\subsubsection{STORM microscopy}

\section{Setup}

Images were recorded using an inverted TIRF microscope (Olympus IX-71) equipped with an oilimmersion objective lens (Olympus, UApoN, 100x magnification, NA 1.49, TIRF) and three lasers; a $647 \mathrm{~nm}$ laser (PhoxX 647, $140 \mathrm{~mW}$, Omicron Laserage, Germany) and 473/488 nm lasers (MBLFN-473nm, 500 mW, Changchun New Industries Optoelectronics Tech. Co.; PhoxX 488, 60 mW, Omicron Laserage, Germany). A quad-edge dichroic beam splitter (Di01-R405/488/561/635, Semrock) and a quad-band excitation filter (FF01-446/523/600/677, Semrock) were used to remove the laser light before imaging the fluorescence on an electron multiplying CCD camera (EMCCD; DU-885-CS0-\#VP, Andor) with an effective pixel size of $80 \mathrm{~nm}$. Temperature of camera was $50{ }^{\circ} \mathrm{C}$. Movies typically contained 3,000 - 4,000 images recorded at $30-90 \mathrm{~Hz}$.

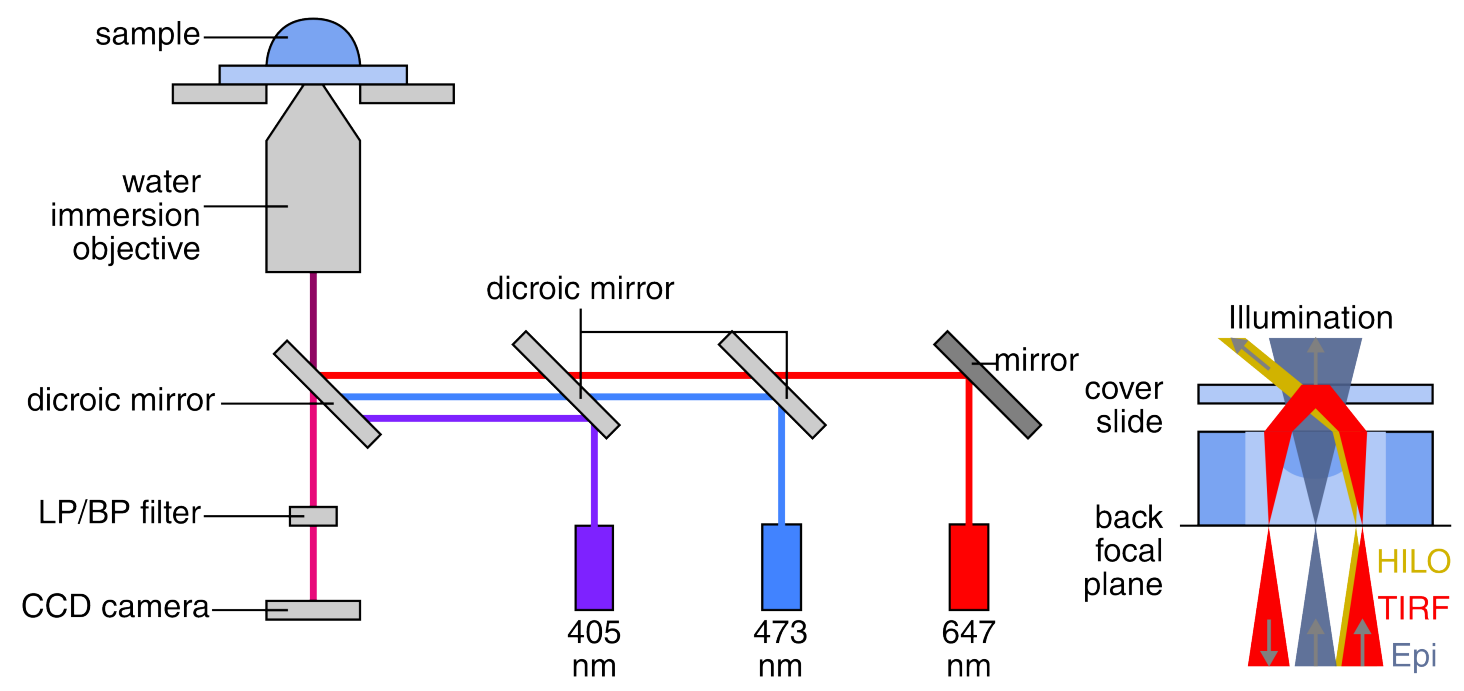

Figure 12: Simple overview of a common fluorescence microscope setup used for STORM imaging. HILO (highly inclined thin illumination) excitation allows for axonal sectioning.

\section{Buffer}

STORM movies were recorded with Alexa 647 labeling. The imaging buffer was 10 mM TRIS containing $100 \mathrm{mM}$ cysteamine hydrochloride (Sigma-Aldrich, M6500), $4.0 \mathrm{mg} / \mathrm{mL}$ glucose oxidase (Sigma-Aldrich, G0543), $0.57 \mathrm{mg} / \mathrm{mL}$ catalase (Sigma-Aldrich, C40-100MG), and $10 \%$ glucose (Sigma-Aldrich, 49158-1KG-F). Buffer was modified and adjusted based on ${ }^{115}[113$ PH was adjusted to the range of $\mathrm{pH} 8.3$ and 8.5 by using $0.3-0.5 \mathrm{M} \mathrm{NaOH}$. Chamber wells were completely filled with buffer and sealed air-free by a regular coverslip. 


\section{Frame rate}

The optimal number of frames used for reconstructing one STORM image was determined by calculating STORM images from different frame numbers. In the progress of this work the number of 3,000 to 4,000 frames was determined as being optimal. Below 3,000 frames per image information are missing. Above 4,000 frames per image no changes are observed. At high frame numbers blinking events get rare and negligible. With an increased recording time, drift increases and resolution decreases. Images at different time points are presented in figure 13 .
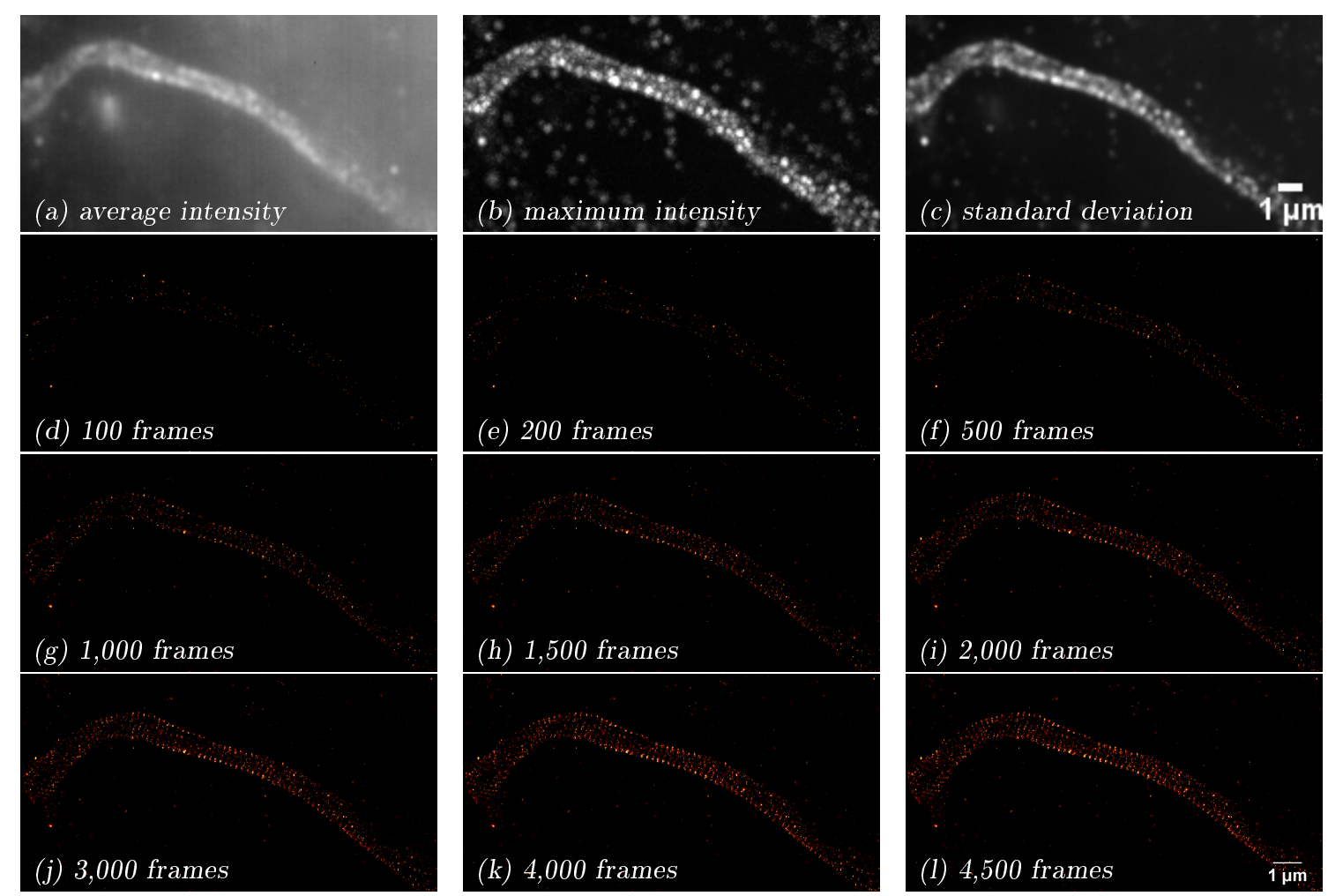

Figure 13: Which frame rate is suitable? The movie was recorded with an exposure time of $0.008 \mathrm{~s}$ and 4,700 frames.

(a) The average intensity of the complete movie. (b) The maximum intensity of the complete movie. (c) The standard deviation of the complete movie. (d) - (1) STORM images calculated from different numbers of frames. Comparison of 2,000 and 3,000 frames shows structural differences, whereas no differences between 4,000 and 4,500 frames are observed. 


\section{Drift}

Stability of the setup was determined by using a zero mode waveguide (ZMW). Nanoholes of different sizes were filled with Alexa 647 diluted $10^{-6}$. Several movies with different exposure times and number of frames were recorded. The recording conditions, identically to the one of the cellular imaging (method: 3.4.2, 3,000 frames with an exposure time of $0.008 \mathrm{~s}$, did not reveal a measurable drift. For 10,000 frames recording with an exposure time of $0.008 \mathrm{~s}$ a drift of about 1.0 pixel, corresponding to $80.0 \mathrm{~nm}$, was determined by principal components analysis (PCA). As a result the drift for cellular STORM images is estimated as 25 to $32 \mathrm{~nm}$.
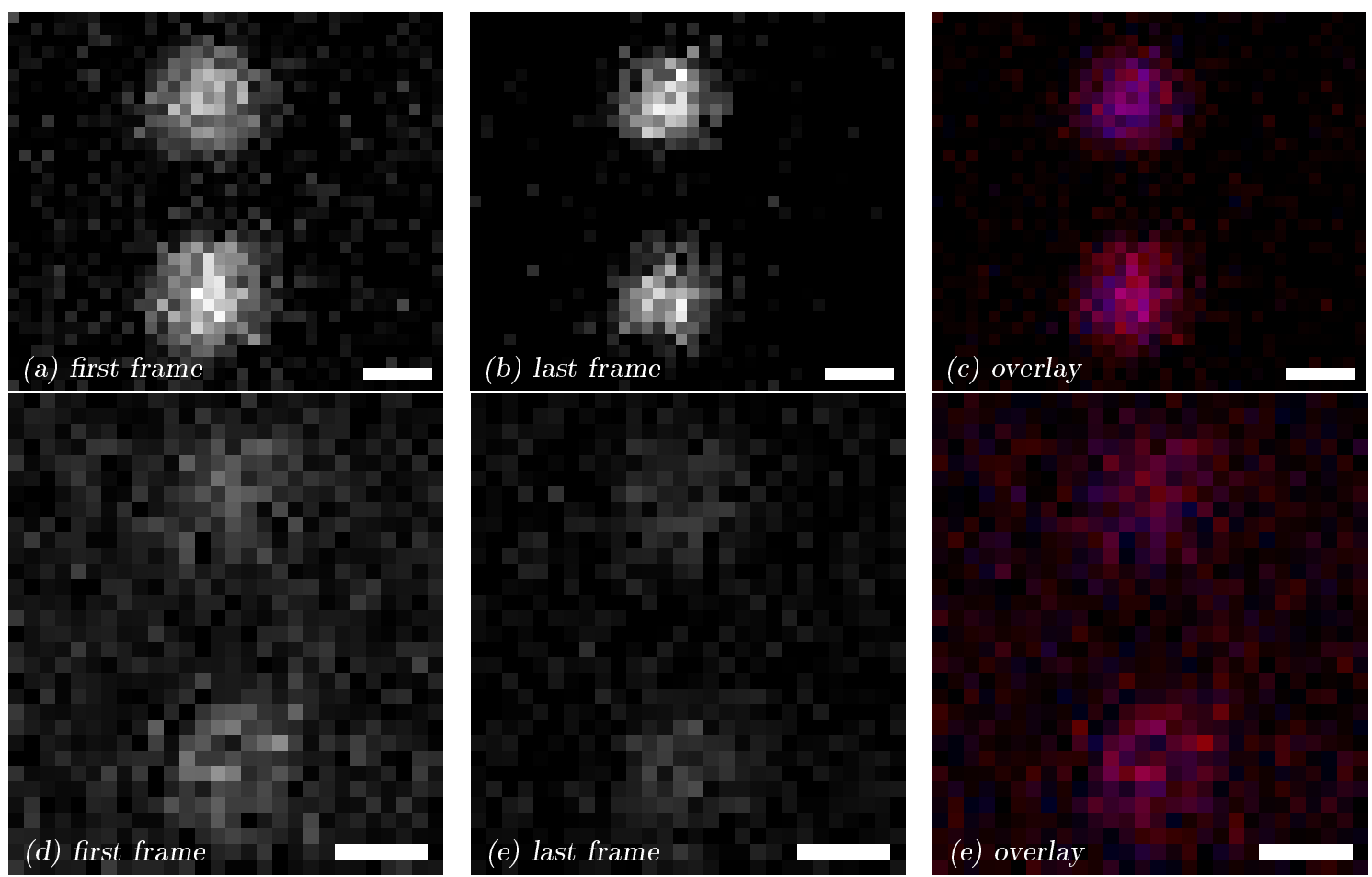

Figure 14: Comparing the first and last frames of movies recorded with an exposure time of $0.008 \mathrm{~s}$. (a) first frame of a 3,000 frame movie. (b) last frame of a 3,000 frame movie (c) overlay of the first (red) and last (blue) frame. (d) first frame of a 10,000 frame movie. (b) last frame of a 10,000 frame movie (c) overlay of the first (red) and last (blue) frame. Scale bar: $0.5 \mu \mathrm{m}$. 


\subsection{Data Analysis}

In the following section the methods used for analyzing wide-field and STORM data are described.

\subsubsection{Wide-field microscopy}

\section{Image display}

For visualization colors were assigned to channels and images were merged using ImageJ (software: http://imagej.nih.gov/ij/).

\section{Profiles along the axon initial segment}

Mean fluorescence intensities were calculated along the AIS using custom written Matlab code. Start of analysis was the soma or first branching point. A segmented line was drawn along the axon until the next branching point or its fluorescence diminishes indicating the end of the AIS. The length $\left\|\vec{S}_{i}\right\|$ of segment $i$ was determined by subtracting the segment ending $\vec{r}_{i+1}$ from its starting point $\vec{r}_{i}$,

$$
\vec{S}_{i}=\vec{r}_{i+1}-\vec{r}_{i}=\left(\begin{array}{c}
\Delta x_{i} \\
\Delta y_{i}
\end{array}\right)
$$

The intensity along the $i$ th line segment is now given by $I\left(\vec{r}_{i}+\lambda \vec{S}_{i}\right)$, with $\lambda=[0 \ldots 1]$.

For averaging over the AIS width, a normalized orthogonal vector for each line segment, $\vec{S}_{i}^{\perp}$, was constructed by swapping $\mathrm{x}$ and $\mathrm{y}$ coordinates and inverting $\mathrm{y}$,

$$
\vec{S}_{i}^{\perp}=\frac{\left(\begin{array}{c}
-\Delta y_{i} \\
\Delta x_{i}
\end{array}\right)}{\left\|\left(\begin{array}{c}
-\Delta y_{i} \\
\Delta x_{i}
\end{array}\right)\right\|}, \vec{S}_{i}^{\perp} \vec{S}_{i}=0 .
$$

To average the line segment intensity over five pixels width, corresponding to circa $1.3 \mu \mathrm{m}$, the orthogonal vector was multiplied by $-2,-1,0,1$ and 2 and added to the current line segment position $\vec{r}_{i}+\lambda \vec{S}_{i}$. The averaged intensity over the five pixel width is then

$$
I_{\mathrm{avg}}=\frac{\sum_{j=-2}^{2} I\left(\vec{x}_{i}+\lambda \vec{S}_{i}+j \vec{S}_{i}^{\perp}\right)}{5}, \lambda=[0 \ldots 1]
$$

The mean background was determined at an area not occupied by a neuron and subtracted from the complete profile. For the line profiles, the calculated intensities were averaged and plotted as a function of the distance in $\mu \mathrm{m}$ from the soma or first branching point. A cutoff was made at latest at $70.0 \mu \mathrm{m}$ in distance. The error, plotted as a light area along the profiles, represents the standard error of the mean (SEM). For double labeling e.g. sodium channel subtype $\mathrm{Na}_{\mathbf{v}} 1.2$ 
with Alexa 488 and ankG at its C-terminus with Alexa 647, the segmented line has been drawn in the ankG channel (marker channel) and projected on the $\mathrm{Na}_{\mathrm{v}} 1.2$ channel, in order to ensure the analysis of an exactly identically area.

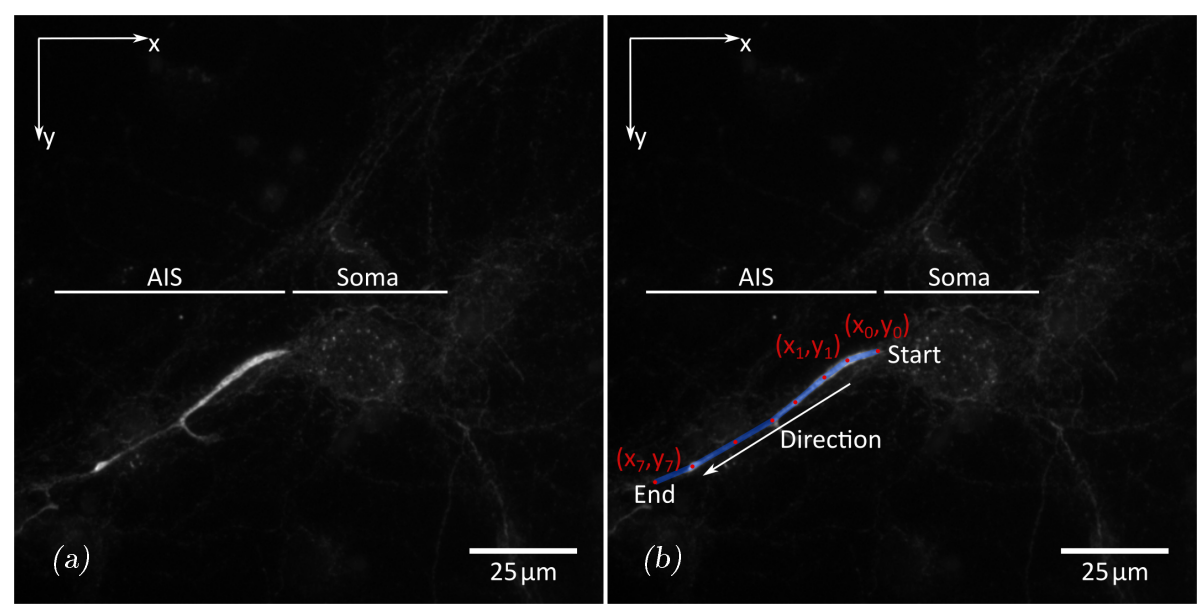

Figure 15: Example for analysis of wide-field images. Pixel location is described by $\mathrm{x}$ and $\mathrm{y}$. Identification of soma and AIS. Determine a START (x, y) and END (x7, y7) point of the AIS. Arrow shows direction of analysis. (a) A hippocampal neuron with its soma and AIS. (b) A segmented line (blue) is drawn along the AIS. Segment pieces are shown in red (points).

\section{Bargraphs of mean fluorescence intensity}

For bargraphs the mean fluorescence intensity along each AIS was calculated for a distance of $50.0 \mu \mathrm{m}$ starting from the soma or first branching point. The background of a non-occupied area was determined and subtracted. The average of the region was calculated and plotted using Matlab. The error is the SEM.

\section{Cell-by-cell analysis}

From every neuron profiles along the AIS were drawn as described above. The maximum intensity of every profile was determined, $\max _{A I S}$. The mean fluorescence intensity of a defined somatic region was determined, mean soma $_{\text {For }}$ Fevy neuron, the maximum fluorescence intensity of the profile was divided by the somatic mean fluorescence intensity. All obtained values $(N)$ were summed and averaged for each maturation stage in order to calculate a factor.

$$
\text { factor }=\frac{\sum_{i=1}^{N}\left(\max _{\mathrm{AIS}} / \operatorname{mean}_{\mathrm{soma}}\right)}{N}
$$




\subsubsection{STORM microscopy}

\section{Image display}

The final STORM images were reconstructed by using the Software rapidSTORM $[122$. For Alexa 647 the PSF FWHM was set as $250 \mathrm{~nm}$. The intensity threshold was chosen as a fixed value of 1,000 ("fixed global threshold"). For image display a fixed minimum localization strength ("minimum localization strength") between 4,000 and 8,000 was chosen. Localizations below this threshold were discarded (details: $\frac{122}{122}$ ).

\section{Profiles along the axon initial segment}

Relative localization strength profiles were extracted from the AISs using ImageJ (software: http://imagej.nih.gov/ij/). Averaging was performed over ten pixels in thickness, corresponding to $100.0 \mathrm{~nm}$.

Plotting of profiles was performed in Matlab.

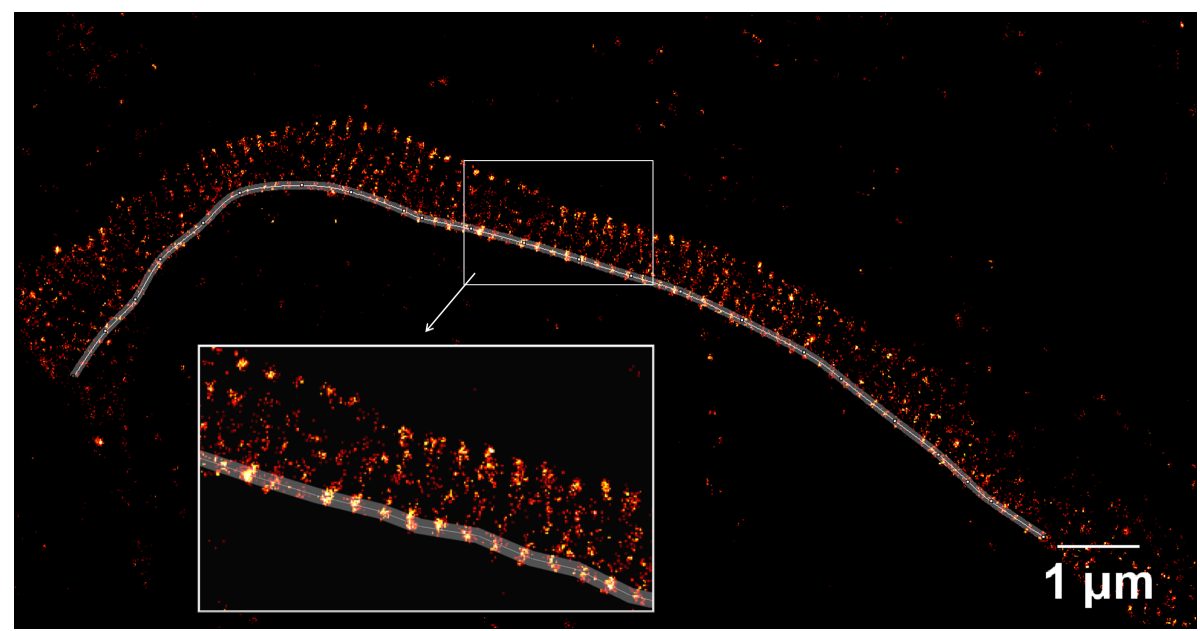

Figure 16: Example for analysis of STORM images. Ten pixel thick segmented lines are drawn along the sides of the AISs. The mean average localization strengths of the ten pixels along the full AIS are extracted. $1.0 \mu \mathrm{m}$ long example profiles are plotted. Full length data are saved for Fourier analysis (methods: 3.5 .2 .

\section{Power spectra}

The power spectra $P(k)$ were extracted from all line profiles individually by evaluating the amplitude square of their respective Fourier transforms. Each set of power spectra were then averaged as described below. An error estimate for the error of the mean (SEM) for each averaged profile could be determined from the standard deviation directly, due to the stochastical independence of the power spectra.

Applying the discrete Fourier transform on the $i$ th line-profile of length $L_{i}$ results in a Fourier 
spacing $\Delta k_{i}=\frac{1}{L_{i}}$. To average over line profiles with different Fourier spacing $\Delta k_{i}$, a very fine grid with $\Delta k=\frac{\Delta k_{\min }}{a}$, where $\Delta k_{\min }$ is the smallest Fourier spacing in the respective set of power spectra and $a$ is a factor $>1$ that ensures sufficient oversampling (in our case $a=5$ ), was chosen. To obtain the values of the power spectra at the new grid points based on the finer $\Delta k$ spacing, linear interpolation was used. Linear interpolation was sufficient in this case, because even the coarsest grid spacing observed in the data sets is much finer than the discussed features in the power spectra.

This averaging method was preferred over the "sliding window" technique to avoid any bias from different window lengths of the line profiles while accounting for the full amount of data.

For comparison of different power spectra, normalized power spectra $\tilde{P}$ were used, where the normalization was performed over the complete frequency space,

$$
\tilde{P}(k)=\frac{P(k)}{\sum_{k} P(k) \Delta k} .
$$
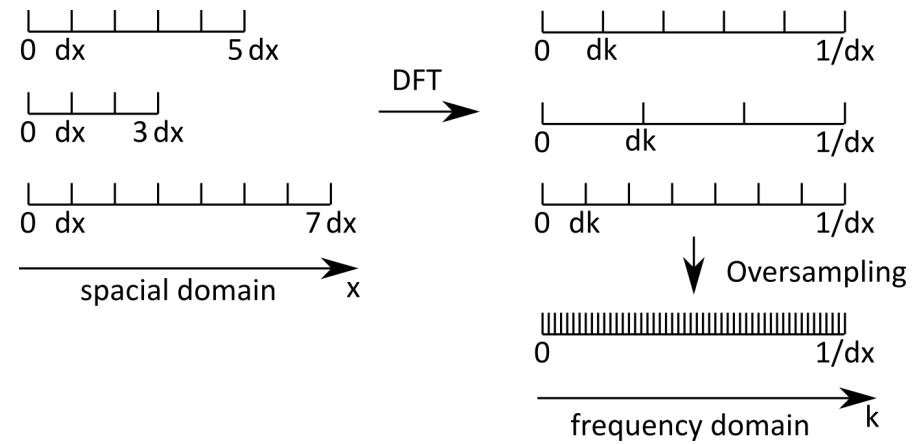

Figure 17: Discrete Fourier transforms of data over different lengths in the spacial domain result in differently spaced frequency domains. Averaging of multiple discrete Fourier transforms is performed via interpolation over an oversampled frequency domain. 


\section{Axon initial segment maturation in hippocampal neurons}

The maturation of the AIS was studied by characterizing the density of the cytoskeletal proteins, $\beta I V$-spectrin and ankG, and VGSCs, including specifically the subtypes $\mathrm{Na}_{\mathrm{v}} 1.2$ and $\mathrm{Na}_{\mathrm{v}}$ 1.6. The protein density was determined as a function of fluorescence intensity in the AIS of neurons in culture by immunohistochemistry and wide-field microscopy. The time of studying neuronal maturation was limited to their electrophysiologically determined mature stage of three weeks.

\subsection{Results}

\section{$\beta I V-s p e c t r i n$, ankG and VGSCs are significantly detected in the AIS.}

A high fluoresence intensity is detected at the AIS, when labeling against $\beta I V-s p e c t r i n$ at a Nand C-terminal region (figure: 19 and 18. A weak dendritic fluorescence intensity is detected. It is about 50 a.u. for N-terminal and about 20 a.u. for C-terminal labeling (determined in week 2, background subtracted).

A high label density is observed at the AIS, when labeling against ankG at a $\mathrm{N}$ - and C-terminal region (figure: 21). A weak, but pronounced dendritic staining is observed by C-terminal labeling. The mean fluorescence intensity at the AIS (figure: 21, DIV 12) is about 13,000 a.u., whereas dendrites have a mean fluorescence intensity of about 3,600 a.u., corresponding to $27.7 \%$ of axonal (discussed in: 9.1.1).

Anti-panNa $\mathrm{v}_{\mathrm{v}}$ labeling reveals a high label density at the AIS (figure: 23). Within the first week the neurons have a low amount of short branches. The AIS is short and not fully developed, but clearly identifiable. PanNa $\mathrm{Pabeling}_{\mathrm{v}}$ is not excluded from the soma and dendrites. Especially, in mature neurons, when the amount and size of branches increase, dendrites are noticeably labeled.

The mean fluorescence intensities detected for $\beta I V$-spectrin, ankG and VGSC labeling at the AISs increase with maturation.

The mean fluorescence intensity of $\beta I V$-spectrin labeled N-terminal doubles from the first to the second week, but does not significantly differ between the second and third week in culture. The mean fluorescence intensity of $\beta I V$-spectrin labeled C-terminal increases significantly between the first and second, but also second and third week (profiles: 34a). Both $\beta I V$-spectrin antibodies reveal a peak in the fluorescence profile at about 10 to $12 \mu \mathrm{m}$ from the soma or first branching point. The peak is more pronounced by C-terminal labeling. The area of a detected high mean fluorescence is about $30 \mu \mathrm{m}$ long. 
The mean fluorescence intensity of ankG labeled N- and C-terminal increases from the first to the second and third week of maturation. It peaks in the range of 10 to $12 \mu \mathrm{m}$ from the soma or first branching point (profiles: 22). Afterward the intensity decreases until it reaches a constant value at about 36 to $38 \mu \mathrm{m}$.

The mean fluorescence intensity of panNa $\mathrm{v}_{\mathrm{v}}$ labeled neurons increases from the first to the second and third week (profile: 24). It reaches a peak at about 10 to $13 \mu \mathrm{m}$ and a constant plateau at about 55 to $60 \mu \mathrm{m}$ in distance.

The characteristic course of the profiles derived from $\beta I V$-spectrin, ankG and VGSC labeled AISs does not shift in distance from the soma or first branching point with maturation.

Normalization reveals that the area of mean fluorescence intensity increase and decrease found in each profile keeps the same distance from the soma or first branching point over the entire three weeks of studied maturation. This implies that its location and size does not change within the time of studied maturation. (figure: 25).

The mean fluorescence intensities of somatic regions and profiles derived from AISs increase simultaneously with maturation.

A detailed cell-by-cell study was performed (as in figure: 27). Thereby, the mean fluorescence intensity of a defined somatic region and the AIS of numerous neurons were determined. The somatic mean fluorescence intensities increase with maturation. This increase is more pronounced between DIV 7 and 14 than between 14 and 21. The difference between the profiles derived from the AISs at DIV 7 and 14 is higher as between 14 and 21 (comparable with the complete averaged data set in figure 24). The trends in the somatic compartment and at the AISs correspond. In both cases the mean fluorescence intensities increase. The factor of the mean maximal fluorescence intensity at the AIS versus the soma is found in the range 8.97:1 for $D I V 7$ and 4.98:1 for $D I V$ 21/21. From DIV 7 to 20/21 the proportion of AIS versus soma channel density decreases.

Sodium channel subtype $\mathrm{Na}_{\mathrm{v}} 1.2$ is detected early on. Sodium channel subtype $\mathrm{Na}_{\mathrm{v}} 1.6$ is not detected before the second week of neuronal maturation.

For sodium channel subtype $\mathrm{Na}_{\mathrm{v}} 1.2$ a bright AIS staining is detected from DIV 6 on (figure: 30 . In most cases the subtype $\mathrm{Na}_{\mathrm{v}} 1.6$ is not detected significantly in the AIS within the first week (figure: 28). Exception, in very dense cultures an AIS staining is observed. Starting at DIV 10 a bright AIS staining is detected. The profile is smooth over the entire analyzed distance. Additionally, a bright regional nuclei and weaker somatic staining is detected for all time points of 
studied maturation.

The mean fluorescence intensities detected at the AISs labeled for sodium channel subtypes $\mathrm{Na}_{\mathrm{v}} 1.2$ and $\mathrm{Na}_{\mathrm{v}} 1.6$ increase with maturation.

For sodium channel subtype $\mathrm{Na}_{\mathrm{v}} 1.2$ the mean fluorescence intensity increases from the first to the third week. Within the first two weeks the intensity peaks in the range of $5-20 \mu \mathrm{m}$. In the third week the mean fluorescence intensity constantly decreases with distance from the soma or first branching point (figure: 31 .

For sodium channel subtype $\mathrm{Na}_{\mathrm{v}} 1.6$ the mean fluorescence profile at $D I V 10$ is relatively weak. The profile is smooth and has no distinct maximum (figure: 29). At DIV 19 and 24 the mean fluorescence is high in the range of about 5 to $55 \mu \mathrm{m}$, but has no distinct peak. It doubles from DIV 10 to 19 . Between DIV 19 and 24 it increases about $300 \%$ within five days. 

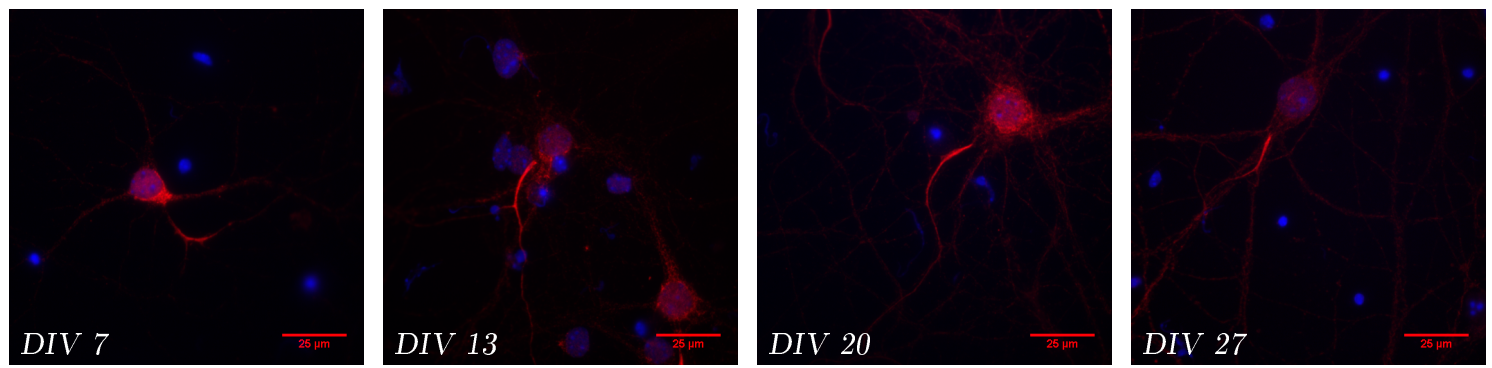

Figure 18: $\beta I V$-spectrin (red) labeled at its N-terminus at $D I V \%, D I V 13, D I V 20$ and $D I V 2 \%$. Nucleus stained by DAPI (blue). Scale bar: $25.0 \mu \mathrm{m}$.
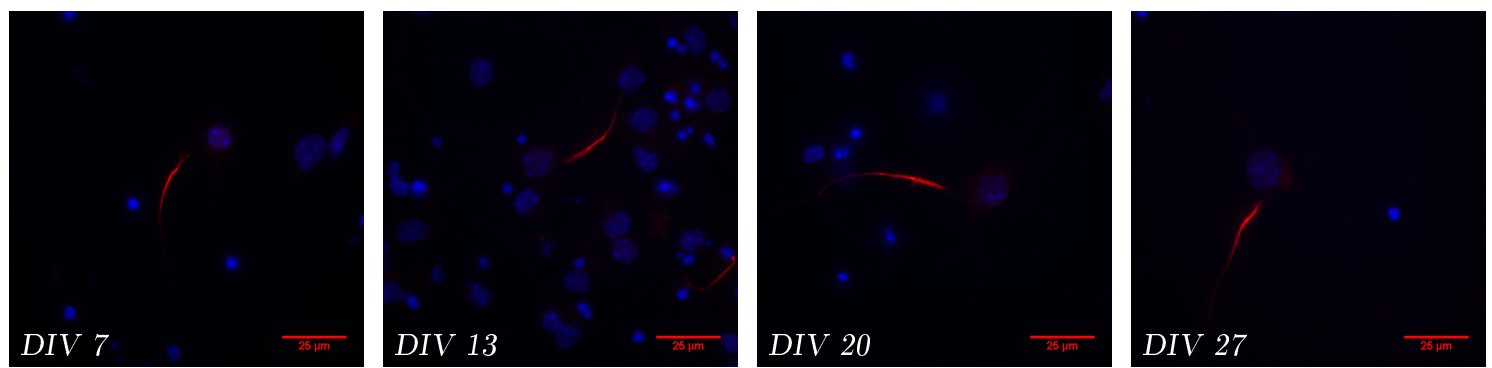

Figure 19: $\beta I V-s p e c t r i n$ (red) labeled at its C-terminus (antibody gift from Rasband lab, Baylor College of Medicine) at DIV \%, DIV 13, DIV 20 and DIV 2\%. Nucleus stained by DAPI (blue). Scale bar: $25.0 \mu \mathrm{m}$.

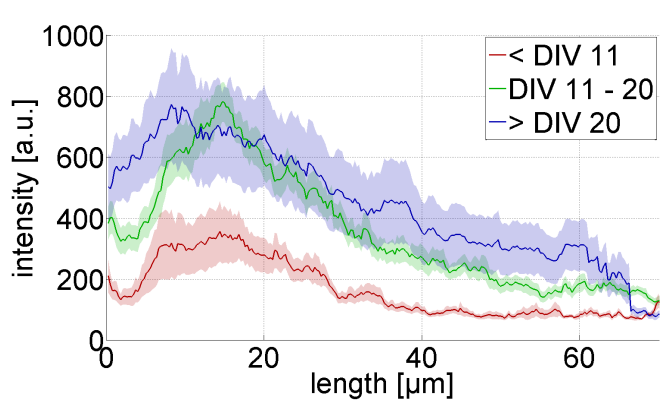

(a) $\beta I V$-spectrin labeled at its N-terminus.

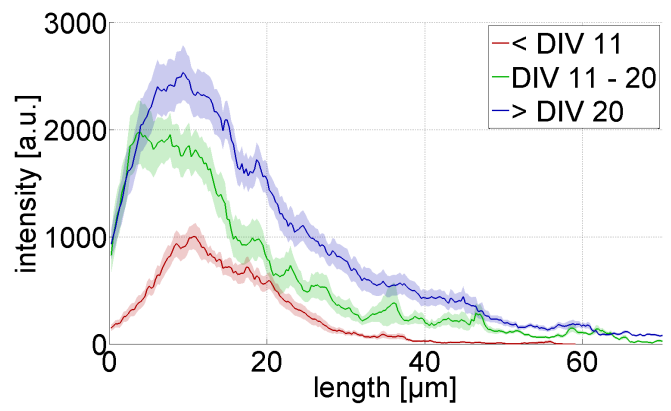

(b) $\beta I V$-spectrin labeled at its C-terminus.

Figure 20: $\beta I V-S p e c t r i n$ in development. Profiles show the mean fluorescence intensities [a.u.] along the AIS as a function of distance from the soma or first branching point at several time points within the first, second and third

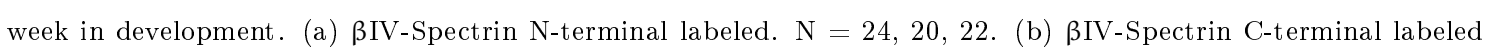
(antibody gift from Rasband lab, Baylor College of Medicine). $\mathrm{N}=24,18,35$. Error is the SEM. 

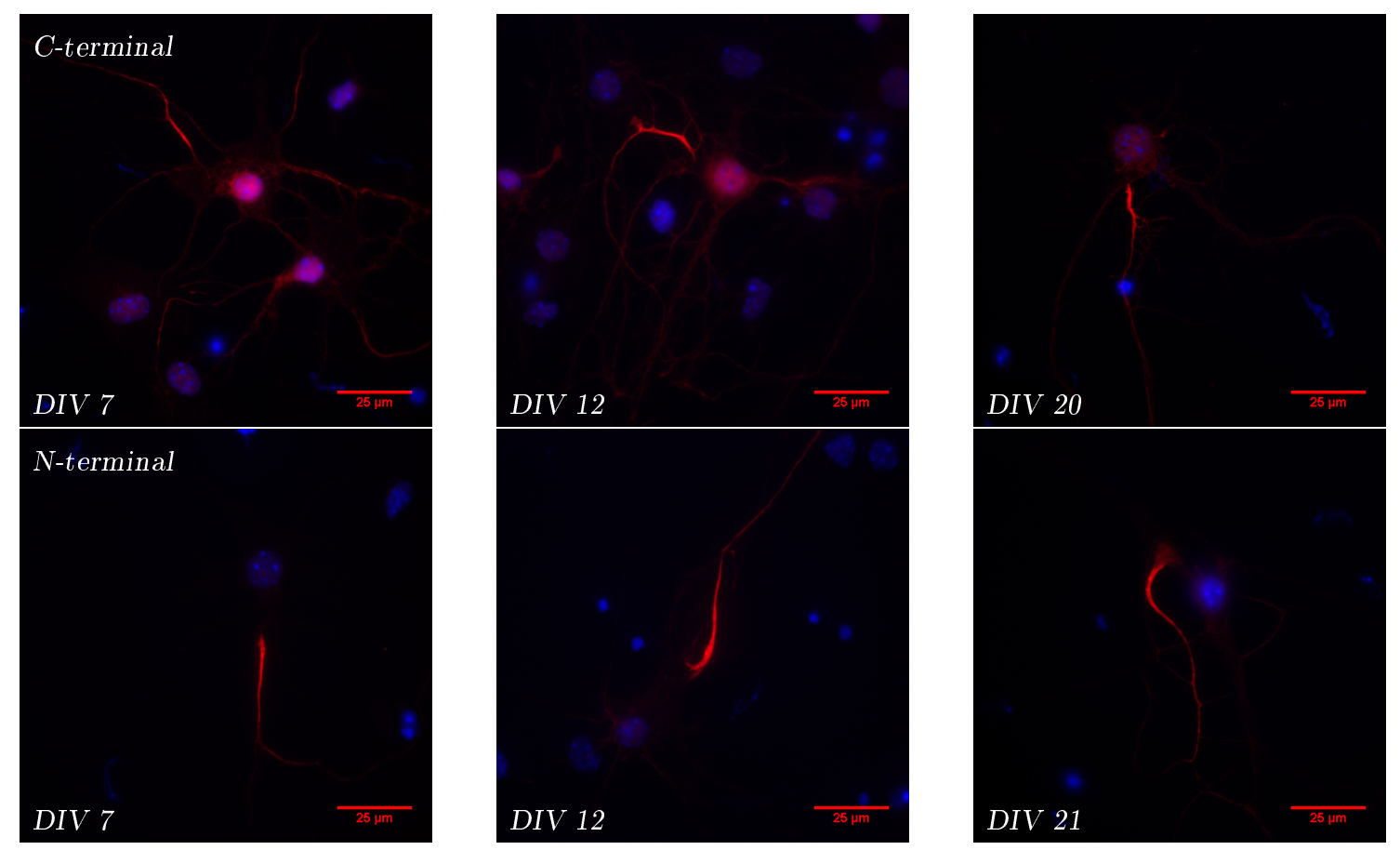

Figure 21: AnkG (red) labeled at its C-terminus (C-terminal antibody, sc-28561, 11.2 (top) and N-terminus (bottom) at DIV 7, DIV 12 and DIV 20/21. Nucleus stained by DAPI (blue). Scale bar: $25.0 \mu \mathrm{m}$.

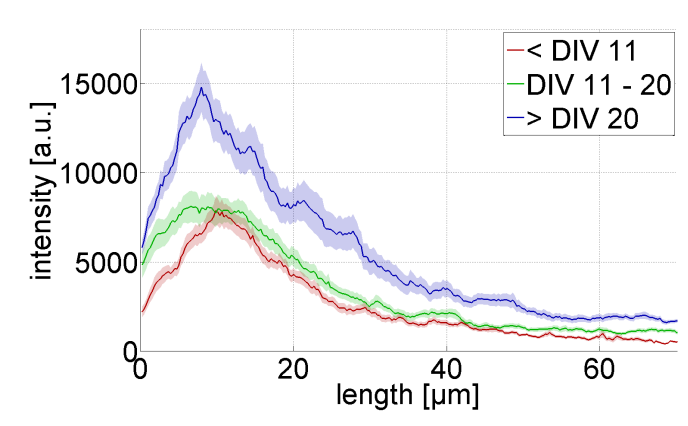

(a) AnkG labeled at its C-terminus.

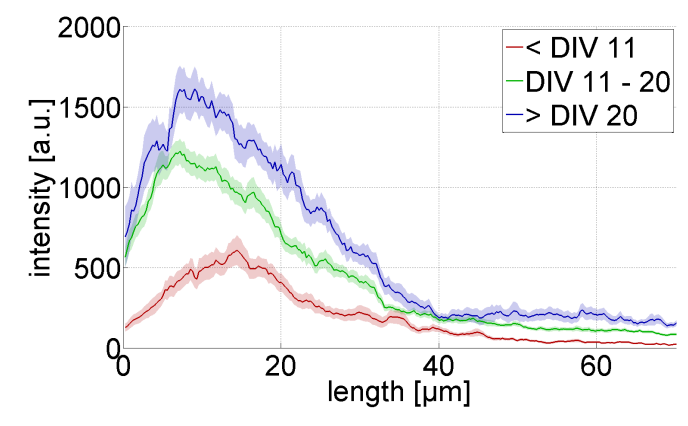

(b) AnkG labeled at its N-terminus.

Figure 22: Ankyrin-G in development. Profiles show the mean fluorescence intensities [a.u.] along the AIS as a function of distance from the soma or first branching point at several time points within the first, second and third week in development. (a) AnkG C-terminal labeled (C-terminal antibody, sc-28561, 11.2. $\mathrm{N}=41,39,37$. (b) AnkG N-terminal labeled. $\mathrm{N}=23,43,26$. Error is the SEM. 

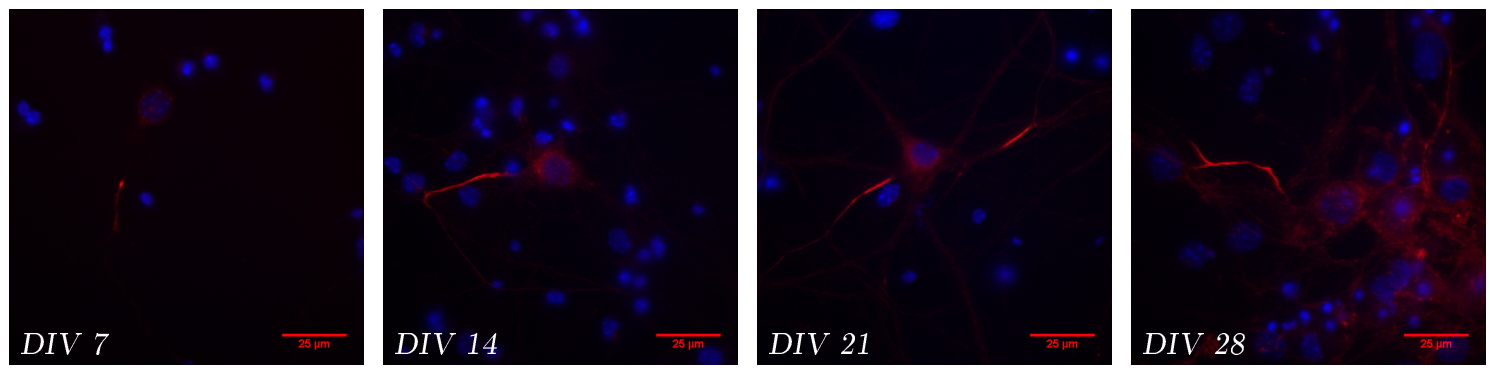

Figure 23: Voltage-gated sodium channels (red) at $D I V$ 7, DIV 14, DIV 21 and $D I V 28$. Nucleus stained by DAPI (blue). Scale bar: $25.0 \mu \mathrm{m}$.

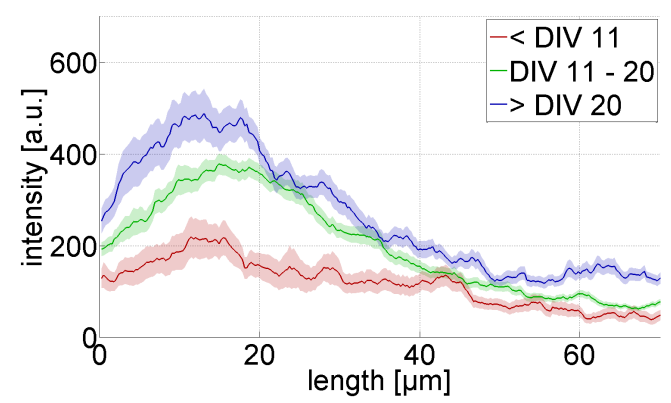

(a) All voltage-gated sodium channel subtypes: Mean fluorescence intensities [a.u.] along the AIS.

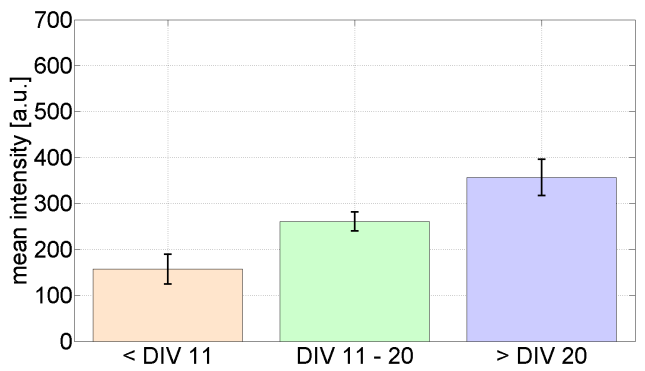

(b) All voltage-gated sodium channel subtypes: Mean fluorescence intensities [a.u.] at the AIS within the distance $0-50.0 \mu \mathrm{m}$ from the soma or first branching point.

Figure 24: Voltage-gated sodium channels in development. (a) Profiles show the mean fluorescence intensities [a.u.] along the AIS as a function of distance from the soma or first branching point at several time points within the first, second and third week in development. (b) Bargraphs show the mean fluorescence intensities [a.u.] at the AIS within the distance $0-50.0 \mu \mathrm{m}$ from the soma or first branching point within the first, second and third week in development. $\mathrm{N}=39,168,73$. Error is the SEM. 


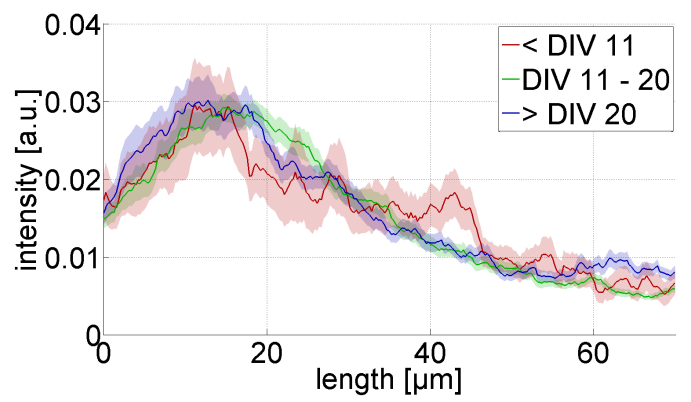

Figure 25: Voltage-gated sodium channels in development. The question if VGSCs change their relative position in the AIS with maturation is determined by normalizing the area below the obtained mean profiles (results: 24) to one. The courses of the profiles do not change and the profiles do not shift within the three weeks of studied maturation. The region of high mean fluorescence intensity does not change with maturation. Error is the SEM.

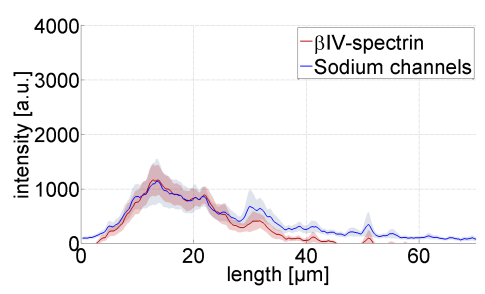

(a) $D I V 8$

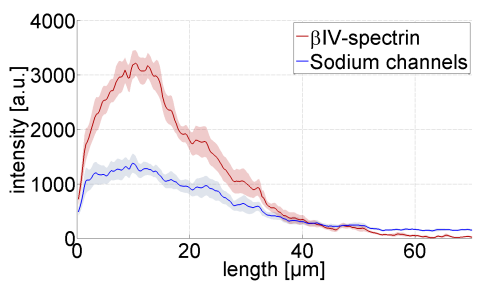

(c) DIV 14

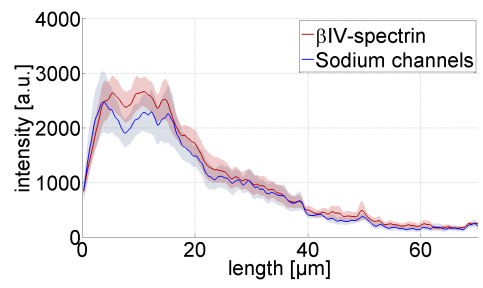

(e) $D I V 21$

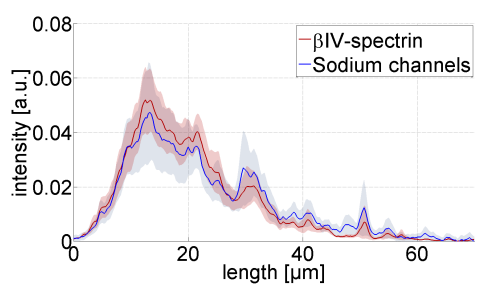

(b) normalized $D I V 8$

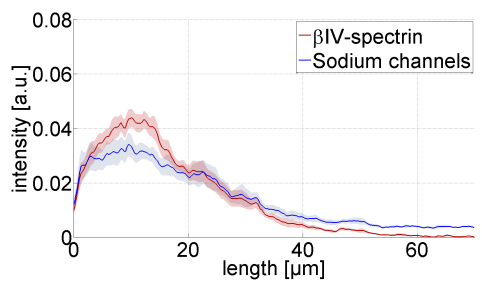

(d) normalized DIV 14

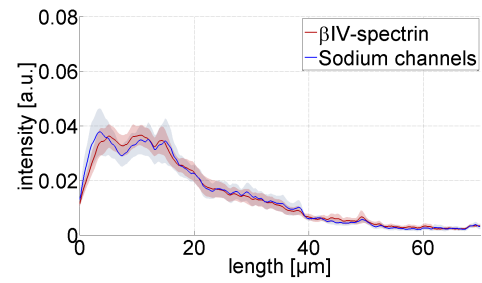

(f) normalized $D I V 21$

Figure 26: Voltage-gated sodium channels and $\beta I V$-spectrin colocalize in maturation. Double stainings of VGSCs and $\beta \mathrm{IV}$-spectrin (antibody gift from Rasband lab, Baylor College of Medicine) were performed. Profiles were extracted at three time points: (a) Profiles at DIV 8, (c) Profiles at DIV 14, (e) Profiles at DIV 21. Normalization of the profiles reveals a similar course and areas of high mean fluorescence intensity: (b) Normalized profiles at DIV 8, (d) Normalized profiles at DIV 14. (f) Normalized profiles at DIV 21. N = 10,11, 12. Error is the SEM. 


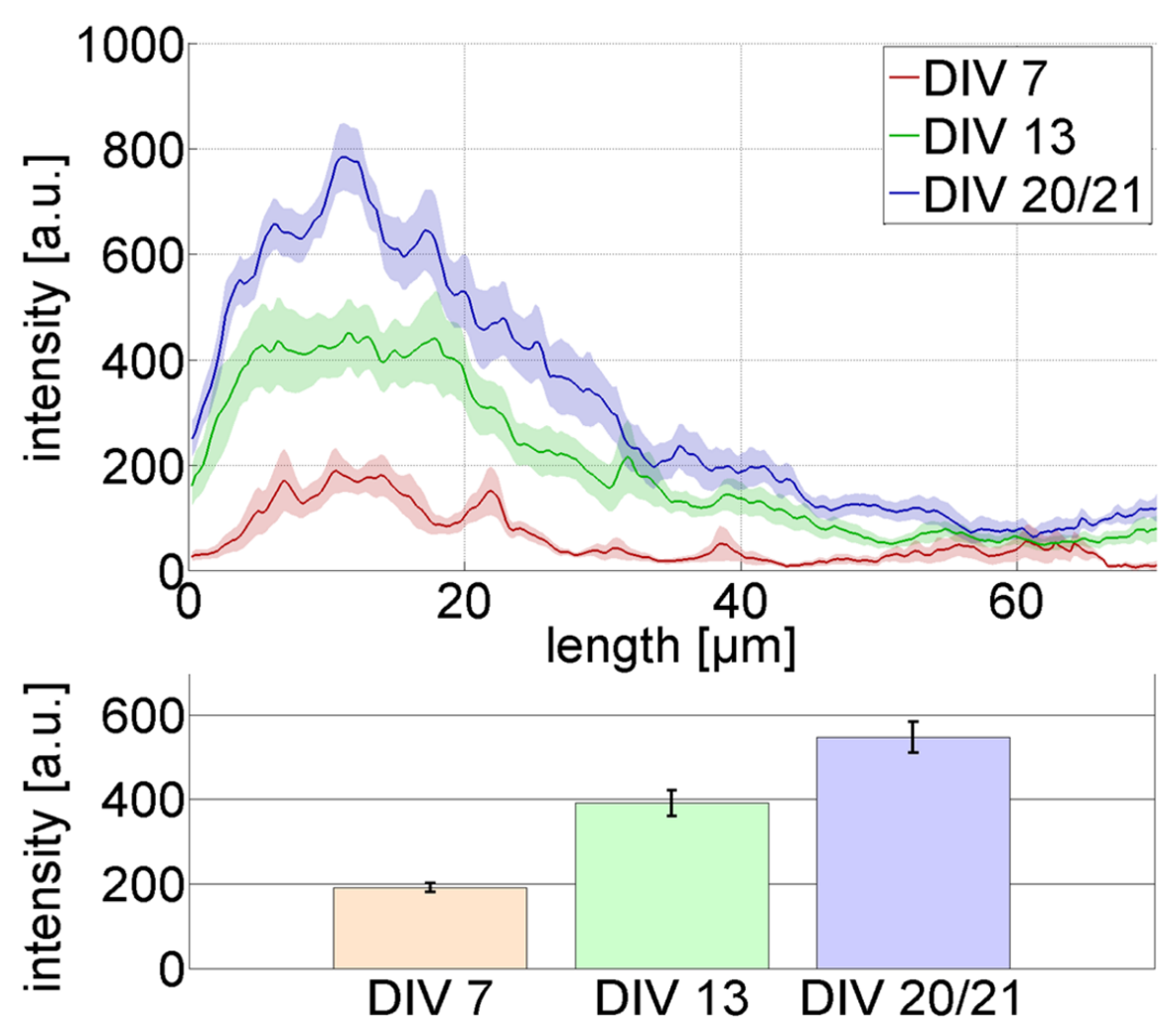

Figure 27: Voltage gated sodium channels labeled by anti-panNa $\mathrm{v}_{\mathrm{v}}$ and analyzed cell-by-cell in the AIS and soma. Neurons are DIV 7, DIV 13 and DIV 20/21. (Top) Profiles show the mean fluorescence intensities [a.u.] along the AIS as a function of distance from the soma or first branching point at three time points. (Bottom) The mean fluorescence intensities [a.u.] for defined somatic regions of the neurons are plotted as bars. The factor of the maximum mean intensity along the AIS versus mean intensity at the soma $($ factor $=$ intensity $($ mean max. (AIS)/ mean (soma)) ) is 8.97 at DIV \%, 7.86 at DIV 13, and 4.98 at DIV 20/21. N $=6,8,10$. Error is the SEM. 

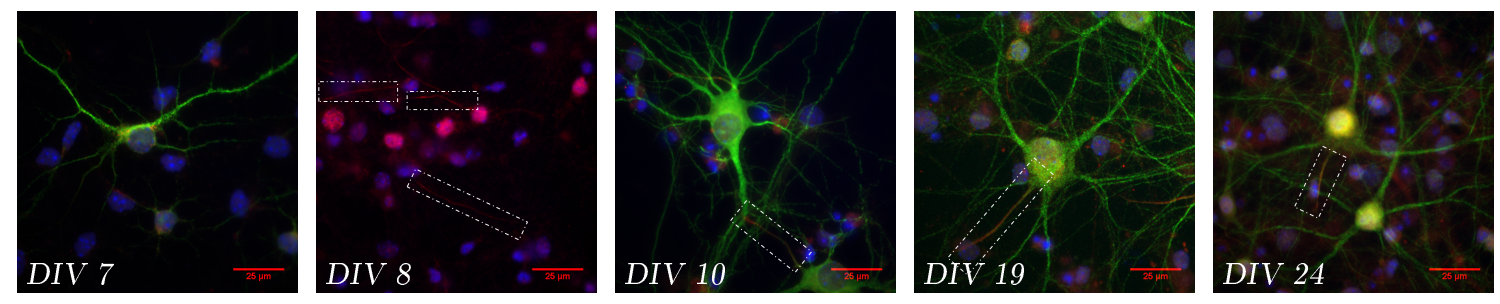

Figure 28: Voltage-gated sodium channel subtype $\mathrm{Na}_{\mathrm{v}} 1.6$ (red) (boxed) at DIV 7, DIV 8, DIV 10, DIV 19 and $D I V$ 24. DIV 7 shows a sparse culture, no significant labeling of subtype $\mathrm{Na}_{\mathrm{v}} 1.6$ at the AIS. DIV 8 shows a dense culture and a significant labeling of subtype $\mathrm{Na}_{\mathrm{v}} 1.6$ at the AIS. At DIV 10 AISs are mostly $\mathrm{Na}_{\mathrm{v}} 1.6$ positive. Double labeled with the marker MAP2 (green) at DIV 10, 19 and 24 highlights the AIS. Nucleus stained by DAPI (blue). Scale bar: $25.0 \mu \mathrm{m}$.

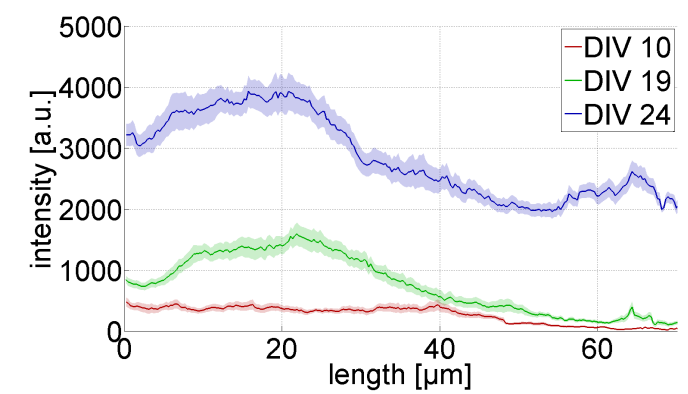

(a) Sodium channel subtype $\mathrm{Na}_{\mathrm{v}} 1.6$ : Mean fluorescence intensities [a.u.] along the AIS.

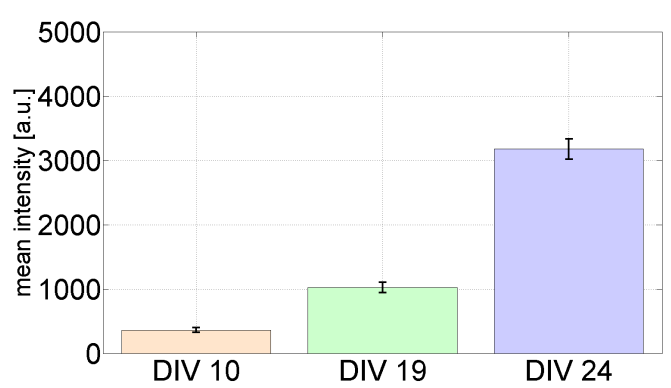

(b) Sodium channel subtype $\mathrm{Na}_{\mathrm{v}} 1.6$ : Mean fluorescence intensities [a.u.] at the AIS within the distance 0 $50.0 \mu \mathrm{m}$ from the soma or first branching point.

Figure 29: Voltage-gated sodium channel subtype $\mathrm{Na}_{\mathrm{v}} 1.6$ in development. (a) Profiles show the mean fluorescence intensities [a.u.] along the AIS as a function of distance from the soma or first branching point at several time points within the second, third and fourth week in development. (b) Bargraphs show the mean fluorescence intensities [a.u.] at the AIS within the distance $0-50.0 \mu \mathrm{m}$ from the soma or first branching point within the second, third and fourth week in development. $\mathrm{N}=15,22,33$. Error is the SEM. 


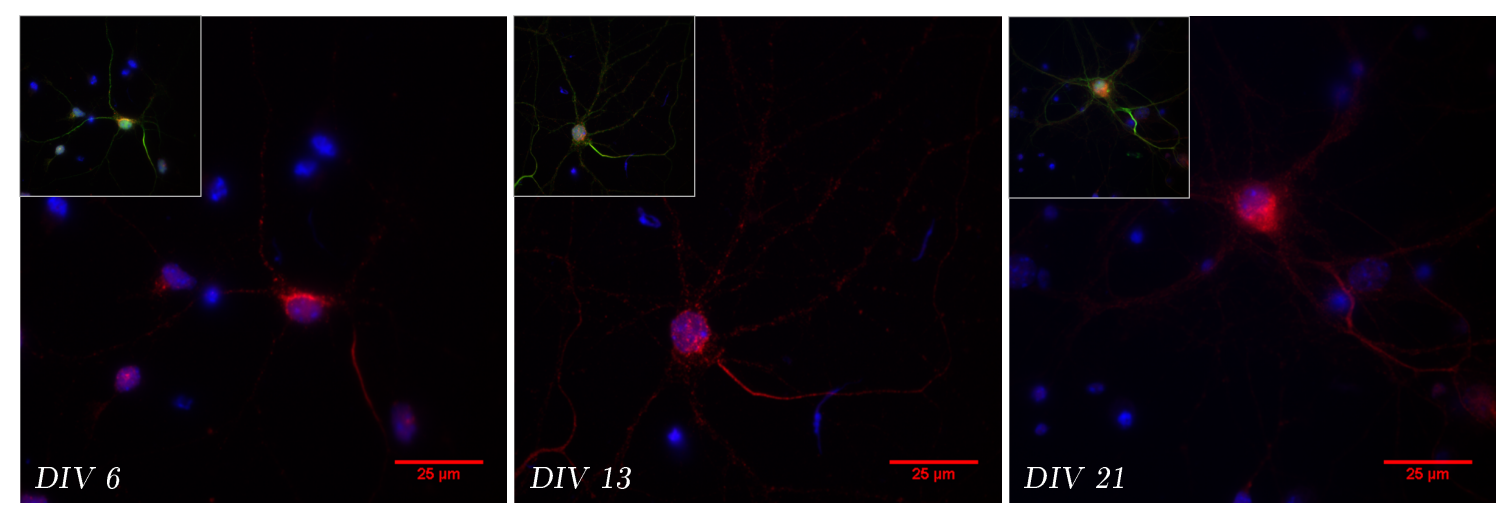

Figure 30: Voltage-gated sodium channel subtype $\mathrm{Na}_{\mathrm{v}} 1.2$ (red) at DIV 6, DIV 13 and DIV 21. Top left: Neurons are shown in double labeling with the marker ankG (labeled at its C-terminus, sc-28561, 11.2) (green) for the identification of the AIS. Nucleus stained by DAPI (blue). Scale bar: $25.0 \mu \mathrm{m}$.

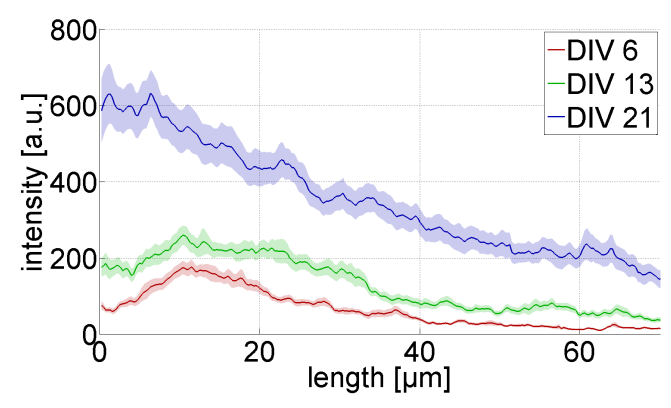

(a) Sodium channel subtype $\mathrm{Na}_{\mathrm{v}} 1.2$ : Mean fluorescence intensities [a.u.] along the AIS.

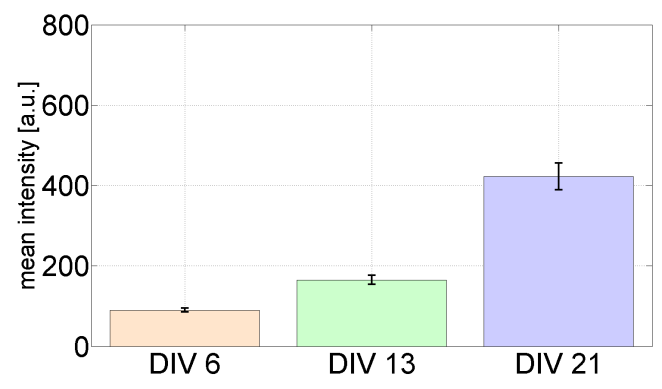

(b) Sodium channel subtype $\mathrm{Na}_{\mathrm{v}} 1.2$ : Mean fluorescence intensities [a.u.] at the AIS within the distance 0 $50.0 \mu \mathrm{m}$ from the soma or first branching point.

Figure 31: Voltage-gated sodium channel subtype $\mathrm{Na}_{\mathrm{v}} 1.2$ in development. (a) Profiles show the mean fluorescence intensities [a.u.] along the AIS as a function of distance from the soma or first branching point at several points within the first, second and third week in development. (b) Bargraphs show the mean fluorescence intensities [a.u.] at the AIS within the distance $0-50.0 \mu \mathrm{m}$ from the soma or first branching point within the first, second and third week in development. $\mathrm{N}=26,24,34$. Error is the SEM. 


\subsection{Discussion}

$\beta I V$-spectrin, ankG, and VGSC are enriched in the AIS and mature simultaneously. $\beta I V$-spectrin, ankG and VGSCs are significantly detected in the AIS from the first week on. It is concluded that the proteins are highly enriched in the AIS (as described before: $76,0,170$, 17 . Profiles, which report the mean fluorescence intensities along the AISs for three mature stages, reveal an increase of the mean fluorescence intensity with maturation. This allows to conclude that the detected proteins increase in their density in the AIS with maturation. Since the mean fluorescence intensity between the second and third week of maturation still increases, it is likely that the proteins are not fully expressed within the three weeks of studied maturation. Exception, $\beta I V$-spectrin labeled N-terminal reports a doubling of the mean fluorescence intensity from the first to the second week, but no significant difference between the second and third week. This antibody suggests that the majority of $\beta I V-$ spectrin is placed in the AIS network in early maturation.

Normalization (details: 4.1) of the profiles reports that none of the proteins shifts in distance from the soma or first branching point within the three weeks of studied maturation. Thus, a shift of the localization of the proteins along the AIS with maturation is unlikely. $\beta$ IV-spectrin, ankG, and VGSCs are likely to be stabilized within a defined region.

The coexpression and overlapping placement in the AIS of $\beta I V$-spectrin, ankG and VGSCs support the role of $\beta I V$-spectrin and ankG to stabilize and local enrich VGSCs within a defined region in the AIS. 86

Chronological expression and differential roles of $\beta I V$-spectrin isoforms - $\beta$ IV $\sum 1$ and $\beta I V \sum 6$.

N-terminal labeling of $\beta I V$-spectrin shows no significant increase of the mean fluorescence intensity after the second week. C-terminal labeling shows a constant increase of the mean fluorescence intensity over the entire three weeks. Consequently, as mentioned above, does the N-terminal antibody suggest that the majority of $\beta I V$-spectrin is placed in the AIS in early maturation, whereas the C-terminal antibody does not suggest a certain maturation stage, where $\beta I V$-spectrin is mainly placed in the AIS network. This controversy was examined more closely by performing database research and double labeling experiments on neurons with the $\mathrm{N}$ - and C-terminal antibodies.

The database research revealed that the spectrin splice variant 1 , referred to as $\beta \operatorname{IV} \sum 1$, is targeted by the N- and C-terminal antibody. Only the C-terminal antibody targets an epitope located in $\beta I V \sum 6$. Additionally, the double labeling experiments prove that $\mathrm{N}$ - and C-terminal antibodies target different $\beta I V$-spectrin isoforms (results: 59, 60. Together these findings suggest that $\beta \operatorname{IV} \sum 1$ appears early, whereas $\beta \operatorname{IV} \sum 6$ appears later in maturation. Late expression of $\beta \operatorname{IV} \sum 6$ has 
been described before. ${ }^{16}$ Moreover, the results confirm $\beta \operatorname{IV} \sum 1$ as a main stabilizer, as described in: 14] [15] [18]. Further, the late expression of $\beta \operatorname{IV} \sum 6$ suggests that this isoform might play a major role in VGSC recruitment and even stabilization in the AIS with neuronal maturation.

$\mathrm{Na}_{\mathrm{v}} 1.2$ and $\mathrm{Na}_{\mathrm{v}} 1.6$ channels are enriched in the AIS and their density increases with maturation. The channel subtypes are enriched at different regions along the AIS. A matural switch from $\mathrm{Na}_{\mathrm{v}} 1.2$ to $\mathrm{Na}_{\mathrm{v}} 1.6$ channels is not found.

Some cultures $(D I V 8)$ displayed neurons, which are strongly positive for $\mathrm{Na}_{\mathrm{v}} 1.6$ channel labeling, whereas other cultures $(D I V 7)$ revealed not a single neuron with an AIS positive for $\mathrm{Na}_{\mathrm{v}} 1.6$ channel labeling. These results suggest the absence and enrichment of $\mathrm{Na}_{\mathrm{v}} 1.6$ channels in young neurons. One explanation is the culture density. In most cases no AISs positive for $\mathrm{Na}_{\mathrm{v}} 1.6$ channel labeling were found in the first week. The few cultures, which displayed neurons with AISs positive for $\mathrm{Na}_{\mathrm{v}} 1.6$ channels in the first week, are very dense. This supports previous observations that neurons in very dense culture mature faster. Furthermore, due to high culture density, these cultures cannot be studied over three weeks of maturation.

Finally at $D I V$ 10, a significant fluorescence intensity is observed at the AIS of all neurons. The mean fluorescence profile has a low intensity and appears smooth (figure: 29). This suggests that the $\mathrm{Na}_{\mathrm{v}} 1.6$ channels are weakly expressed and evenly distributed along the AIS in early maturation. The high mean fluorescence intensity increase between $D I V 19$ and 24 allows to conclude that the density of $\mathrm{Na}_{\mathrm{v}} 1.6$ channels highly increases. Moreover, $\mathrm{Na}_{\mathrm{v}} 1.6$ channels are mainly found in the AIS of mature neurons (as described before: [99] At DIV 19 and 24 the mean fluorescence intensity profiles reveal areas of higher and lower intensity, which suggest that $\mathrm{Na}_{\mathrm{v}} 1.6$ channels are not evenly distributed in the AIS of mature neurons.

$\mathrm{Na}_{\mathrm{v}} 1.2$ are detected in the first week of studied maturation. This allows to conclude that $\mathrm{Na}_{\mathrm{v}} 1.2$ channels are expressed early on, as described before: 98 . A doubling of the intensity from one to another week suggests that the density of $\mathrm{Na}_{\mathrm{v}} 1.2$ channels increases in mature neurons.

In immature neurons an intensity peak at about $10 \mu \mathrm{m}$ from the soma or first branching point reports $\mathrm{Na}_{\mathrm{v}} 1.2$ enrichment. In mature neurons the intensity profile of $\mathrm{Na}_{\mathrm{v}} 1.2$ decreases constantly and no intensity peak is observed. Together the findings reveal a proximal $\mathrm{Na}_{\mathrm{v}} 1.2$ and distal $\mathrm{Na}_{\mathrm{v}} 1.6$ localization in the AIS, as described before: ${ }^{31}$. Moreover, a prominent proximal $\mathrm{Na}_{\mathrm{v}} 1.2$ enrichment can be associated with immature neurons and distal $\mathrm{Na}_{\mathrm{v}} 1.6$ enrichment can be associated with mature neurons.

The analog increase of $\mathrm{Na}_{\mathrm{v}} 1.2$ and $\mathrm{Na}_{\mathrm{v}} 1.6$ densities with maturation suggests that there is no replacement of $\mathrm{Na}_{\mathrm{v}} 1.2$ by $\mathrm{Na}_{\mathrm{v}} 1.6$ with maturation, as observed in noR. 8 


\section{The density of voltage-gated sodium channels increases in somatic regions with maturation.}

The mean fluorescence intensity detected by an $\mathrm{panNa}_{\mathrm{v}}$ antibody was found to increase in somatic regions with maturation. Anti-panNa $\mathrm{v}_{\mathrm{v}}$ targets all VGSC subtypes. The subtypes $\mathrm{Na}_{\mathrm{v}} 1.2$ and $\mathrm{Na}_{\mathrm{v}} 1.6$ have been found as the main subtypes in the AIS. $31 \sqrt{25}$ However, this does not exclude their presence in somatodendritic compartments, as described before ${ }^{27}$. Additionally, other VGSC subtypes, including $\mathrm{Na}_{\mathrm{v}} 1.1$ and $\mathrm{Na}_{\mathrm{v}} 1.3$ have been reported in somatodendritic compartments of central neurons (including pyramidal neurons). $1223[6963$ Here, an increase of the somatic fluorescence intensity is found and allows to conclude that at least one VGSC subtype increases in its density in the somatic compartment of hippocampal neurons with maturation.

\section{No tremendous exceeding of VGSCs in the AIS.}

A careful cell-by-cell study with anti-panNa $a_{v}$ labeled neurons was performed. Single neurons were analyzed, whereby the factor from the mean maximal fluorescence intensity at the AIS versus the soma was determined. The relation of the mean fluorescence intensity derived from the AIS and soma is in the range $4.98: 1$ to $8.97: 1$. This suggests a 5 to 9 fold difference between the somatic and AIS VGSC density depending on the matural stages.

Compared with recent studies on differences between AIS and somatic VGSCs, it is difficult to estimate the relevance of the obtained values. Both, low ( 3 fold $)^{[2}$ and high (35 to 80 fold $)^{26}[27$ differences of the VGSC densities between the AIS and soma have been reported. Hereby, the finding of a 5 to 9 fold difference would support the theory that the VGSC density in the AIS has no tremendous exceed over the somatic densities. Nevertheless, studies revealing more striking differences cannot be ignored. Hereby, one needs to consider that the studies have been performed by using different methodical approaches. Whereas a small 3 fold difference has been reported by a combined electrophysiology-imaging study, a high 35 to 80 fold difference has been reported based on immunogold labeling experiments. 26$] 27$ Hereby, methodical drawbacks need to be taken into account. In electrophysiological experiments a patch pipette is used, whereby a part of the membrane is sucked in the pipette, likely that VGSCs lose their connection to the cytoskeleton and the number of VGSCs is underestimated. Freeze fracture experiments with immunogold labeling are not free of false-positive detections. Furthermore, it is unknown if the P-face plasma membrane, used to determine non-specific binding, has the same properties and is unrestricted suitable to determine non-specific binding. Besides general experimental challenges ${ }^{[124]}[125$, such as varying results due to antibody non-specificity or slightly different experimental protocols, immunohistochemistry studies are likely to under- or overestimate the VGSC densities based on the accessibility of the epitopes. For example, epitopes in somatic and axonal regions could 
be unequally easy accessed. Furthermore, the choice of the fluorescence imaging technique is critical. The drawback of the here chosen wide-field microscopy technique is a wide focal plane. Consequently, not only fluorescence from a defined area is collected. A calibration to a defined surface area is impossible. Thus, it is likely that the VGSC density is overestimated based on the detected fluorescence intensity. Additionally, the here applied method (description: 3.5.1) to analyze the data based on the maximum intensity of the AIS and mean intensity of the soma is more likely to over- than underestimate the VGSC density in the AIS. Consequently, when considering all drawbacks of the highly sensitive immunohistochemistry experiments (details in chapter: The specificity of immunohistochemistry 9.1), it is unlikely that the here performed cellby-cell study underestimates the VGSC density in the AIS versus the soma tremendously. This allows to conclude that the VGSC density in the AIS relative to the soma is likely to be even smaller as presented here.

For every method, especially immunohistochemistry experiments, a careful evaluation is necessary in order to estimate the sensitivity of the method and understand the quality of the drawn conclusions. A detailed evaluation of each antibody was performed and is found summarized in the supplements (chapter: 9.1). 


\section{Structural organization of axon initial segment components in hippocampal neurons}

Additionally, to the maturation study, a structural characterization of $\beta I V$-spectrin, ankG and VGSCs in wildtype neurons was performed. Thereby, a focus was on the organization of $\beta I V-$ spectrin isoforms, $\beta \operatorname{IV} \sum 1$ and $\beta \operatorname{IV} \sum 6$, and VGSC subtypes, $\mathrm{Na}_{\mathrm{v}} 1.2$ and $\mathrm{Na}_{\mathrm{v}} 1.6$. Neurons were characterized at the age of DIV 11 to 20 using STORM microscopy.

\subsection{Results}

$\beta I V$-spectrin labeled at its $\mathrm{N}$-terminus and at an epitope located centrally on its sequence displays a regular organization.

Recorded images (figure: 32) show a periodically organized structure. Localization strength profiles along the AIS reveal six regular peaks for N-terminal and ten regular peaks for central labeling per $1.0 \mu \mathrm{m}$. A frequency of about $190 \mathrm{~nm}$ is obtained for N-terminal labeling and a doubled frequency of about $91 \mathrm{~nm}$ for central labeling.

$\beta I V$-spectrin labeled at its C-terminus displays no regular organization in mature neurons.

Recorded images (figure: 32) of mature neurons show no organized structure. The localization strength profile reveals six irregular peaks per $1.0 \mu \mathrm{m}$. No distinct frequency is obtained.

From additional experiments, performed on immature neurons ( $D I V 6)$, images of structured AISs were obtained (figure and results: 5.1). Localization strength profiles reveal six regular spaced peaks. The power spectrum has a broad peak with a frequency of 192 to $200 \mathrm{~nm}$. The periodic organization is prominent, but less as observed for N-terminal and central labeling.

AnkG labeled at the spectrin-binding domain is periodically organized.

Images (figure: 34) recorded by N-terminal labeling (spectrin-binding domain) reveal an organized structure of the AIS. The structure is prominent, but less pronounced as the one obtained from $\beta I V$-spectrin labeled at its N-terminus. No organized structure is observed on images recorded by C-terminal labeling. The localization strength profile for N-terminal labeling reveals five regular peaks. The peaks have no clear maximum and several shoulders, showing broad localizations. Both profiles obtained by ankG labeled C-terminal are irregular. The calculated power spectrum reveals a high power in the frequency range 188 to $201 \mathrm{~nm}$ for N-terminal labeling. The peak has two maxima at the frequencies 192 and $196 \mathrm{~nm}$ (and a small shoulder at about $189 \mathrm{~nm}$ ). No distinct 
frequency for C-terminal labeling is obtained.

VGSCs are (semi-)periodically organized in the AIS. Subtype labeling reports diverse results.

The recorded images (figure: 35p display (semi-)periodic organization for VGSCs. Anti-panNa labeling reveals regional highly periodic and non-periodic segments within a single AIS. Images obtained by $\mathrm{Na}_{\mathrm{v}} 1.6$ subtype labeling show structured, but not prominent periodically organized AISs. In contrast, images obtained by $\mathrm{Na}_{\mathrm{v}} 1.2$ appear relative unstructured. Extracted localization strength profiles show broad, but regular spaced localizations for VGSCs and subtype $\mathrm{Na}_{\mathrm{v}} 1.6$. Five localizations per $1.0 \mu \mathrm{m}$ are found. The extracted profile for the subtype $\mathrm{Na}_{\mathrm{v}} 1.2$ shows regions without localizations. This behavior is reflected in the corresponding power spectra. VGSCs have a clear frequency of about $182 \mathrm{~nm}$. The spectrum has low noise. Subtype $\mathrm{Na}_{\mathrm{v}} 1.6$ has a frequency of about $192 \mathrm{~nm}$. The spectrum is more noisy, especially at high frequencies. Subtype $\mathrm{Na}_{\mathrm{v}} 1.2$ has a frequency peak at about $178 \mathrm{~nm}$, but at higher frequencies peaks with the same power are found. The entire spectrum appears highly noisy, especially at high frequencies. For a more detailed analysis, the power spectrum of each neuron labeled for $\mathrm{Na}_{\mathrm{v}} 1.2$ was plotted separately (results: 10.3). The dataset shows two subclasses of spectra: Completely noisy spectra and spectra, which have several frequencies with a high power, including at or close to $200 \mathrm{~nm}$. No neuron has a spectrum with a single peak at or close to the frequency of $200 \mathrm{~nm}$ and a low level of noise (results: 10.3 . 
5. Structural organization of aXon initial Segment COMPONENTS in hippocampal NEURONS
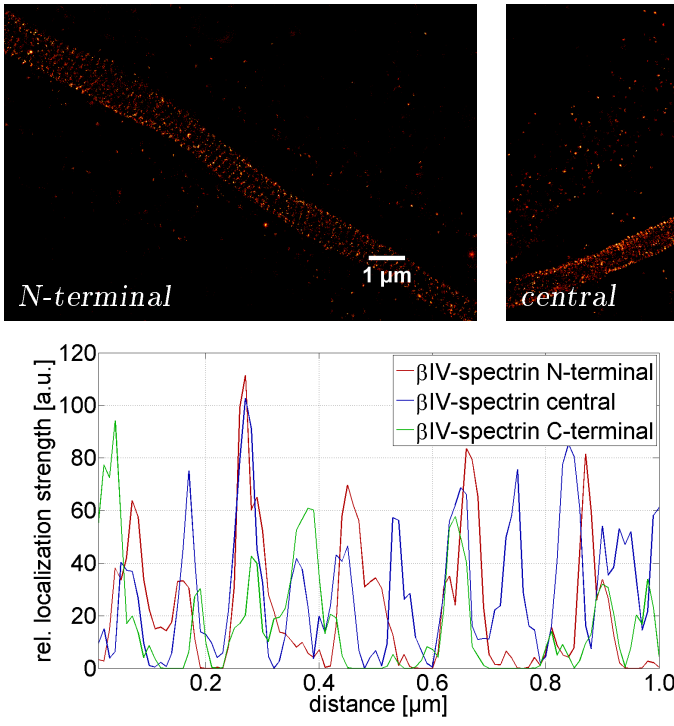
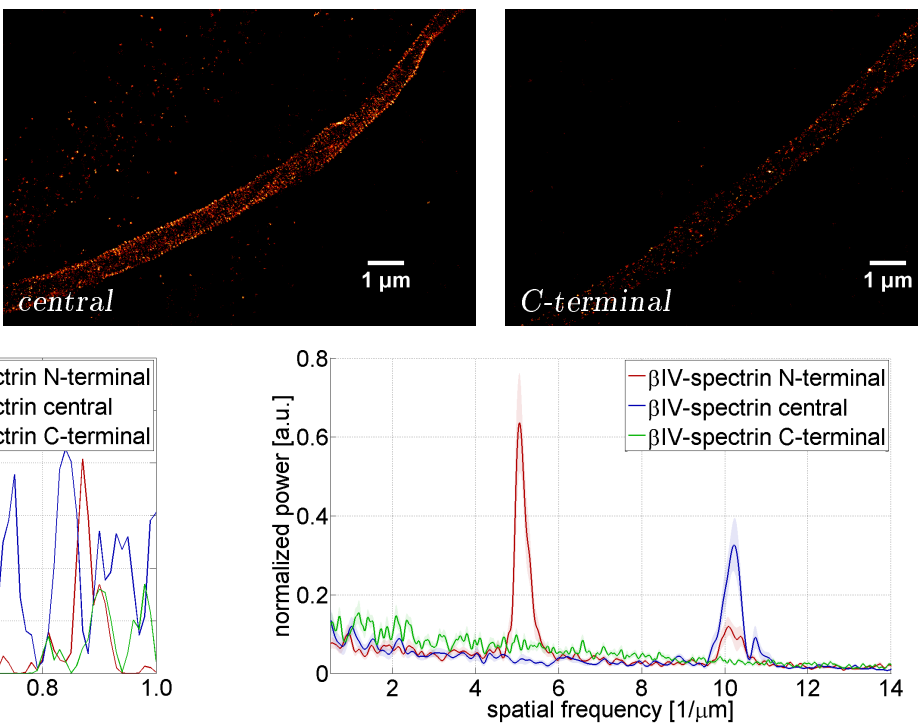

(a) Profiles of $1.0 \mu \mathrm{m}$ segments extracted from the shown (b) Power spectra calculated from the complete $\beta I V-$ neurons.

spectrin data sets.

Figure 32: $\beta I V$-spectrin organization in the AIS imaged by STORM at DIV $11-20$. Example images of the AIS labeled for $\beta I V$-spectrin at an N-terminal, central and C-terminal (C-terminal antibody, sc-131789, 11.2 located epitope. (a) Profiles of $1.0 \mu \mathrm{m}$ segments extracted from the shown neurons show regularly localizations for Nterminal and central labeling and irregular localizations for C-terminal labeling. (b) Power spectra calculated from the complete $\beta I V$-spectrin data sets reveal a periodic organization of about $190 \mathrm{~nm}$ for N-terminal labeling and of about $91 \mathrm{~nm}$ for central labeling. $\mathrm{N}($ for N-terminal, C-terminal and central) $=24,30,18$. Error is the SEM. 
5. Structural Organization OF AXON initial SEGMENT COMPONENTS IN HiPPOCAMPAL
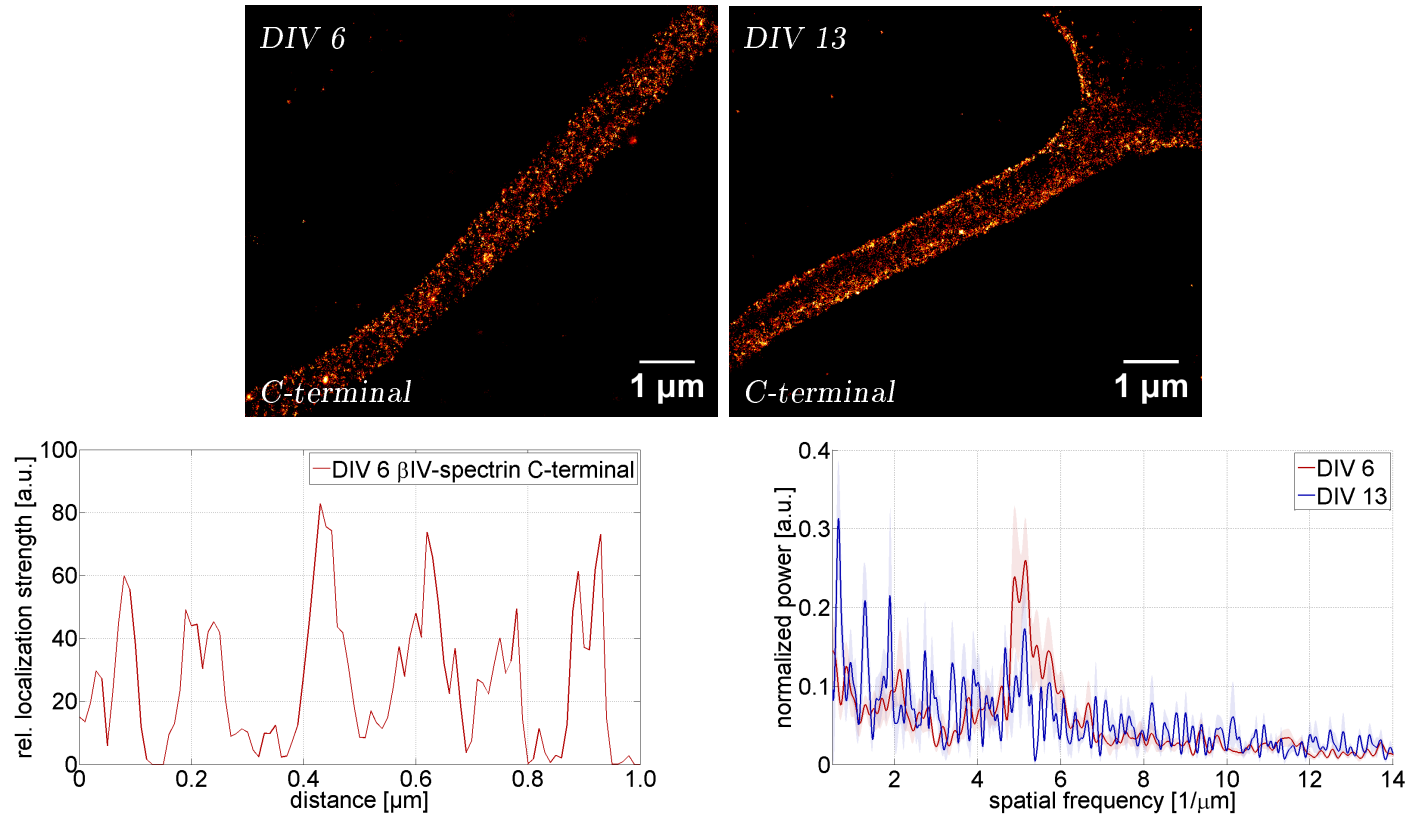

(a) Profile of $1.0 \mu \mathrm{m}$ segment extracted from the shown neuron.

(b) Power spectra calculated from the complete $\beta I V-$ spectrin data sets.

Figure 33: $\beta I V$-spectrin organization in the AIS of immature neurons imaged by STORM at DIV 6 and 13. Example images of the AIS labeled at a C-terminal (antibody gift from Rasband lab, Baylor College of Medicine) located epitope at DIV 6 and 13. (a) The profile of a $1.0 \mu \mathrm{m}$ segment extracted from the shown $D I V 6$ neuron shows regular localizations. (c) Power spectra calculated from the complete data sets reaveal a wide, but clear periodic organization of about 192 to $208 \mathrm{~nm}$ for $D I V 6$ and no organization for DIV 13. $\mathrm{N}$ (for DIV $6, D I V 13)=$ 9, 5. Error is the SEM. 
5. Structural organization of aXon initial Segment COMPONENTS in hippocampal NEURONS
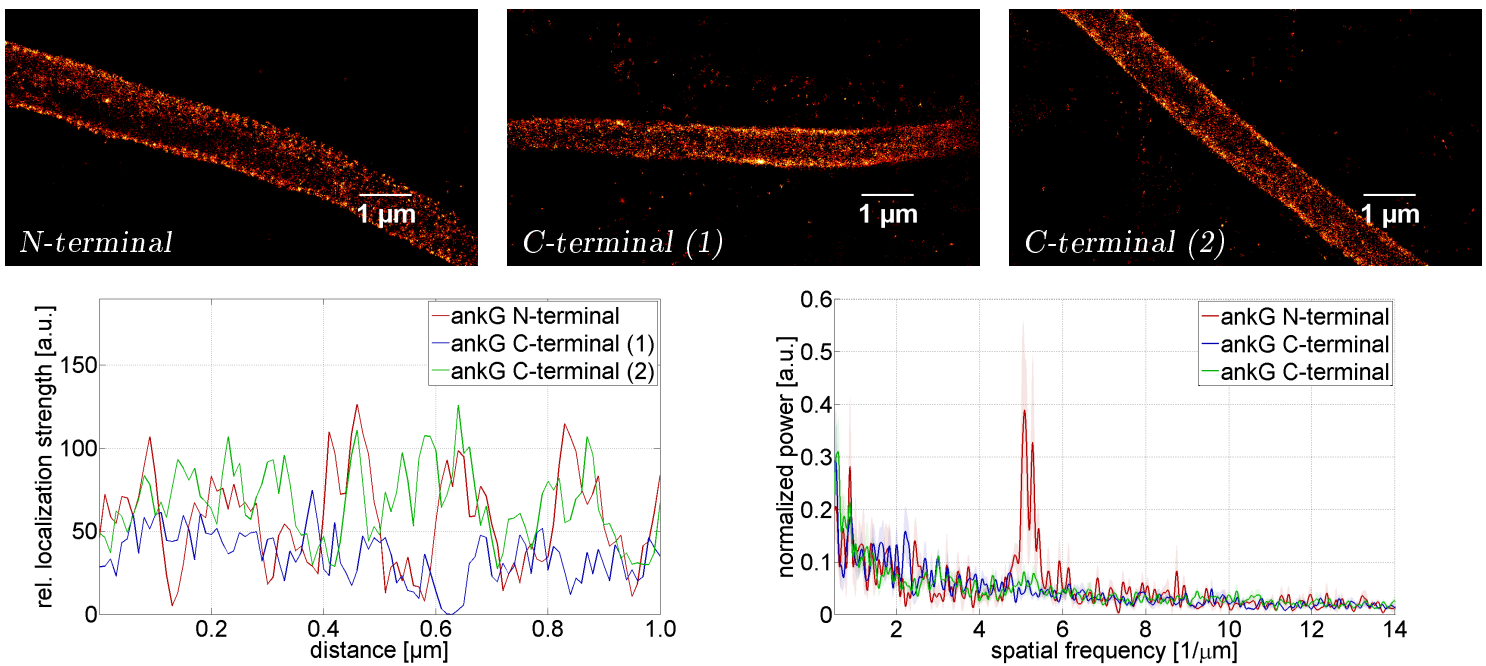

(a) Profiles of $1.0 \mu \mathrm{m}$ segments extraced from the shown (b) Power spectra calculated from the complete ankG neurons.

data set.

Figure 34: AnkG organization in the AIS imaged by STORM DIV 11 - 20. Example images of the AIS labeled for ankG at an N- and two C-terminal located epitopes. (1) C-terminal antibody, sc-28561, 11.2 (2) C-terminal antibody, sc-31778, 11.2 (a) Profiles of $1.0 \mu \mathrm{m}$ segments extracted from the shown neurons show regular localizations for N-terminal and irregularity for C-terminal labeling. (b) Power spectra calculated from the complete ankG data sets reveal a periodic organization in the range of 188 to $201 \mathrm{~nm}$ for N-terminal labeling and no distinct organization for C-terminal labeling. $\mathrm{N}($ for N-terminal, C-terminal (1) and C-terminal (2)) $=5,12,15$. Error is the SEM. 
5. Structural organization of AXON initial SEgment COMPONENTS IN Hippocampal 48 NEURONS
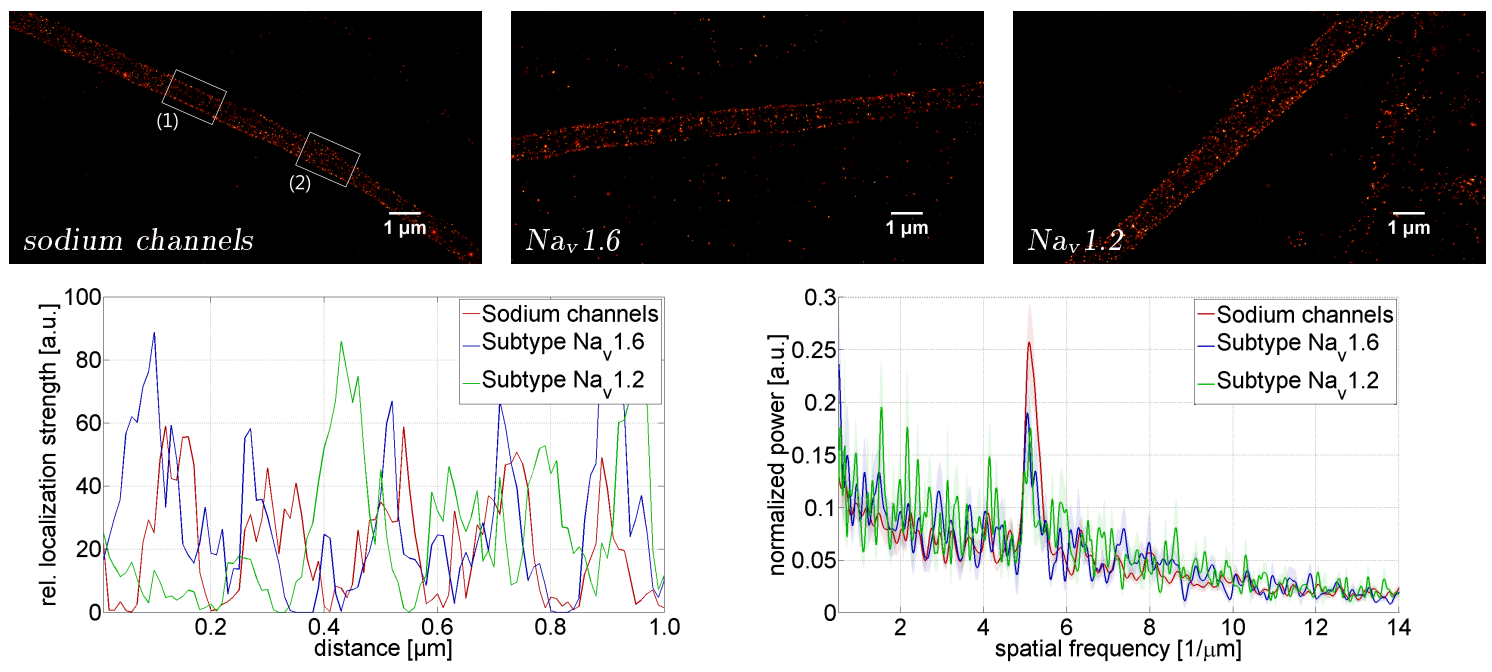

(a) Profiles of $1.0 \mu \mathrm{m}$ segments extracted from the shown

(b) Power spectra calculated from the complete VGSC, neurons.

$\mathrm{Na}_{\mathrm{v}} 1.6$ and $\mathrm{Na}_{\mathrm{v}} 1.2$ data sets.

Figure 35: VGSC organization in the AIS imaged by STORM DIV 11 - 20. Example images of the AIS labeled for VGSCs, subtype $\mathrm{Na}_{\mathrm{v}} 1.6$ only and subtype $\mathrm{Na}_{\mathrm{v}} 1.2$ only. VGSCs show periodically organized areas (Box (1)) and non-periodically organized areas (Box (2)). (a) Profiles of $1.0 \mu \mathrm{m}$ segments extracted from the shown neurons show regular localizations for VGSCs and subtype $\mathrm{Na}_{\mathrm{v}} 1.6$. Irregular localizations are observed for subtype $\mathrm{Na}_{\mathrm{v}} 1.2$. (b) Power spectra calculated from the complete VGSC, $\mathrm{Na}_{\mathrm{v}} 1.6$ and $\mathrm{Na}_{\mathrm{v}} 1.2$ data sets reveal a periodic organization of about $182 \mathrm{~nm}$ for VGSCs and $192 \mathrm{~nm}$ for $\mathrm{Na}_{\mathrm{v}} 1.6$. No clear periodic organization is revealed for $\mathrm{Na}_{\mathrm{v}} 1.2$. N(for VGSCs, $\mathrm{Na}_{\mathrm{v}} 1.6$ and $\left.\mathrm{Na}_{\mathrm{v}} 1.2\right)=36,12,13$. Error is the SEM. 


\subsection{Discussion}

$\beta I V$-spectrin $\mathrm{N}$-terminal labeled reports periodic organization.

For N-terminal labeled $\beta I V$-spectrin a clear spatial frequency of about $196 \mathrm{~nm}$ was obtained. This states that $\beta I V$-spectrin is found as periodically organized when labeled at its N-terminus. The obtained results by N-terminal labeling agree with previously published data, where the periodic structure has been described as $190 \mathrm{~nm}$. (figure: 20 11 Additionally, here the periodic organization is found to be maintained throughout the studied maturation from DIV 11 - 20. To conclude, within the period of studied maturation the $\beta I V$-spectrin N-terminus is highly organized in the AIS and maintains its position.

\section{$\beta I V-s p e c t r i n$ central labeled confirms its tetrameric structure.}

Additionally to previous work, $\beta I V$-spectrin was labeled at an epitope located centrally on the $\beta I V$-spectrin amino acid sequence. A frequency of about $91 \mathrm{~nm}$ was obtained. This frequency corresponds with the tetrameric structure of $\beta I V$-spectrin (figure: 2). Each $\beta I V$-spectrin tetramer consists of two $\beta I V$-spectrin heterodimers. These heterodimers have their N-termini directed towards the actin-adducin rings and form a tetramer through a region close to their C-termini (figure: 2, introduction: 2.2. . Consequently, for $\mathrm{N}$ - and $\mathrm{C}$-terminal targeting the antibody binding positions on two tetramers overlay with each other and cannot be resolved. For centrally located binding regions, two binding positions at one tetramer are resolved, resulting in the doubled frequency of about $91 \mathrm{~nm}$. Together, the findings obtained by N-terminal and central labeling of $\beta I V$-spectrin support the tetrameric structure of the $\beta I V$-spectrin molecule.

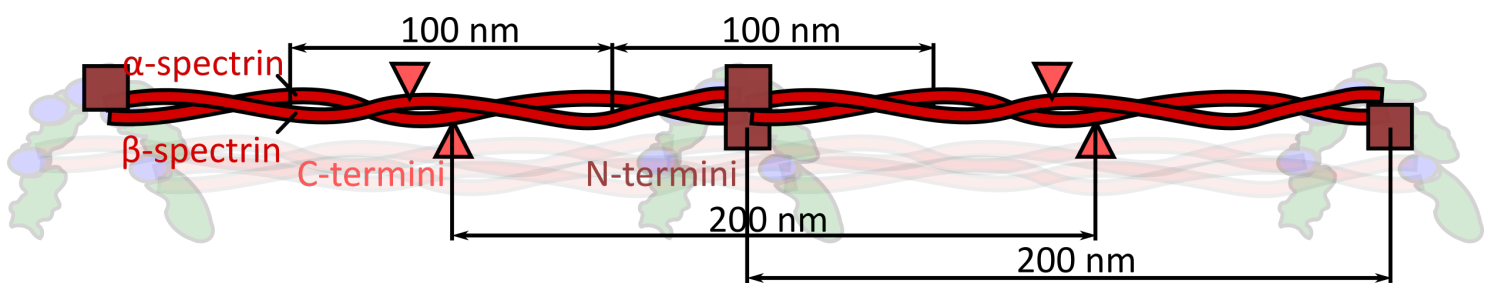

Figure 36: A schematic view of anti- $\beta I V$-spectrin antibodies binding to the $\beta I V$-spectrin tetramers organized in the AIS. N- and C-terminal binding positions are two overlaying positions, one per heterodimer and thus the distance is about $200 \mathrm{~nm}$. Central located binding positions of heterodimers do not overlay and appear with a distance of about $100 \mathrm{~nm}$

\section{$\beta I V$-spectrin C-terminal labeled reports diffuse distribution in mature AIS.}

Based on the tetrameric structure, for C-terminal labeling the same frequency as for N-terminal labeling is expected. Recently, a non-prominent periodic organization in rat neurons (DIV 12) has been published and assumed to be due to a freely moving $\mathrm{C}$-terminal region of $\beta \mathrm{IV}$-spectrin 
molecules within the periodic network. ${ }^{12}$ These findings have not been investigated further.

Here, experiments with immature and mature neurons were performed. $\beta I V$-spectrin labeled Cterminal in immature neurons (results: 5.1, $D I V 6$ ) is found to be periodically organized. In mature neurons no periodic organization is found.

The obtained results suggest $\beta \operatorname{IV} \sum 6$ as a non-periodically organized splice variant appearing in mature neurons.

$\beta I V$-spectrin has multiple splice variants. Database research and double labeling experiments reveal that the $\mathrm{N}$ - and $\mathrm{C}$-terminal antibodies target different splice variants of $\beta I V$-spectrin (results: 60 , whereas $\beta \operatorname{IV} \sum 1$ is targeted by both antibodies and $\beta \operatorname{IV} \sum 6$ only by the C-terminal antibody. $\beta \operatorname{IV} \sum 1$, the main isoform and expressed from early on, ${ }_{16}^{17}$ is organized in a periodic matter, here confirmed by labeling experiments at an N-terminal and central located epitope. $\beta \operatorname{IV} \sum 6$ has been described as appearing later in maturation $\sqrt{16}$. This suggests that a high density of $\beta \operatorname{IV} \sum 1$, but no or only a low density of $\beta \operatorname{IV} \sum 6$, is detected by C-terminal labeling at $D I V 6$. Consequently, similar structures for $\beta I V$-spectrin labeled $\mathrm{N}$ - and $\mathrm{C}$-terminal in young neurons are resolved. In mature neurons an increasing irregularity is detected by C-terminal labeling. It is likely that an increasing density of $\beta \operatorname{IV} \sum 6$ is integrated in the existing network in a non-periodic matter. Summarized, the findings suggest that a non-periodically organized splice variant, $\beta \operatorname{IV} \sum 6$, is the reason for irregular $\beta I V$-spectrin organization detected by C-terminal labeling. Presumably, its non-periodic organization is due to the lack of an actin-binding domain, which is required for the transfer of the periodic organization from the underlying network. ${ }^{[126]}$ The theory of a freely moving C-terminal region of $\beta I V$-spectrin is rejected.

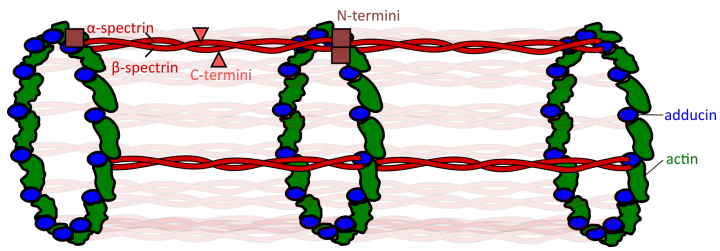

(a) Model of $\beta I V$-spectrin in the AIS of an immature neuron.

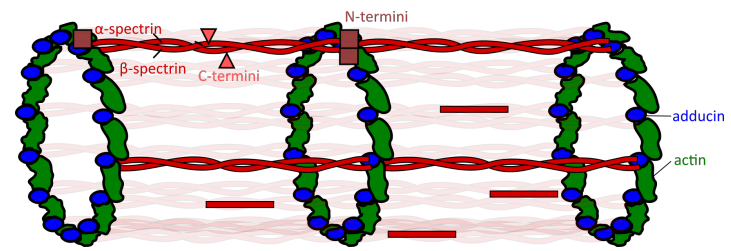

(b) Model of $\beta I V$-spectrin in the AIS of a mature neuron.

Figure 37: Model of $\beta I V$-spectrin organization in the AIS. Spectrin tetramers connect actin-adducin rings with each other. (a) Immature neurons consist of $\alpha \mathrm{II} / \beta \mathrm{IV} \sum 1$-spectrin tetramers, which form a highly organized AIS. (b) Mature neurons consist of $\alpha \mathrm{II} / \beta \mathrm{IV} \sum 1$-spectrin tetramers and the additional splice variant of spectrin, $\beta$ IV $\sum 6$. $\alpha \mathrm{II} / \beta \mathrm{IV} \sum 1$-spectrin tetramers form a highly structural organized AIS. $\beta$ IV $\sum 6$ lacks an actin-binding domain and integrates in the existing network in a non-periodic matter. 


\section{AnkG organization is mediated by binding to the underlying periodically organized} network.

AnkG has a spectrin-binding domain and has been found to bind $\beta I V$-spectrin. 45,72 Consequently, it is likely that the periodic organization of the underlying network is transferred to ankG. (figure: 2). The used N-terminal ankG antibody targets the spectrin-binding domain and thus it is most likely to reveal an organized pattern. Here, its organization was found in a frequency range between 188 to $201 \mathrm{~nm}$. This has been lately described for neurons derived from rats. 12 The identified frequency is not surprising. Three factors are likely to reduce the periodic appearance of ankG (in comparison to $\beta I V$-spectrin). (i) Since ankG is not a part of the underlying periodic network and has a certain distance to the network, it is likely that the periodic organization is not fully transferred and a less pronounced periodic organization is found. (ii) Additionally, ankG has multiple binding positions on a single $\beta I V$-spectrin tetramer, $62[53 \lcm{63}[57[19$ which are located close to each other, but do not overlap. As a consequence, not all available binding sites might be occupied with ankG molecules and not all ankG molecules might be targeted by an antibody (e.g. due to steric hindrance and low affinity). As a result, depending on which positions are targeted and imaged, the distance between two resolved ankG molecules varies, possibly in the range of 160 to $240 \mathrm{~nm}$ (figure: 38). The small distance between two ankG binding positions on a single $\beta I V$-spectrin tetramer cannot be resolved by STORM microscopy (introduction: 2.6), thus further investigations were not feasible. (iii) Further, $\beta \operatorname{IV} \sum 6$ lacks an actin-binding domain [16], but does not lack an ankyrin-binding domain. Thus, especially in mature neurons, where $\beta \operatorname{IV} \sum 6$ is likely to be highly expressed, its non-periodic organization is transferred to ankG and a less pronounced organization of ankG is expected to be found.

AnkG labeled C-terminal is found to be non-periodically organized in mature neurons (results for immature neurones: 10.2). This is in agreement with recent findings made in neurons derived from rats. ${ }^{12}$ The targeted C-terminal epitope has no proximity to the spectrin-binding domain and thus is less likely to reveal a periodic organization based on the underlying network. Especially, when deliberating that giant ankG is a $480 \mathrm{kDa}$ protein and has a length of nearly $150 \mathrm{~nm} . \frac{73}{750} \mathrm{~nm}$ is about $75 \%$ of the length of a $\beta I V$-spectrin tetramer. Consequently, a single ankG molecule has the length of two-thirds of the spacing between two actin-adducin rings connected by $\beta I V$-spectrin tetramers. Thus it is likely that the underlying cytoskeletal periodic pattern could only be revealed by $\mathrm{C}$-terminal labeling if all ankG molecules would be organized in the same direction. 


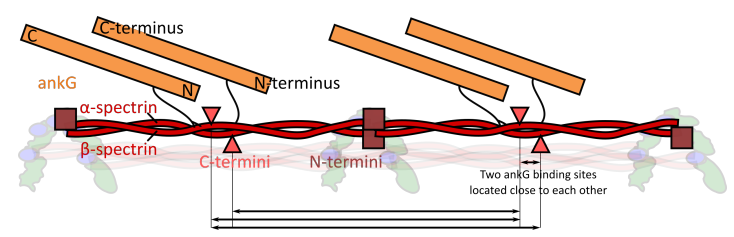

Figure 38: A schematic view of the $\beta I V$-spectrin tetramer and its binding sites to ankG. AnkG binds (here shown with its spectrin-binding domain) close to the C-terminal region of the $\beta I V$-spectrin tetramer. As a consequence two binding positions are located close to each other. AnkG molecules can differ in distance (demonstrated by arrows): Depending on (i) whether all ankG binding positions on the $\beta I V$-spectrin tetramer are occupied or (ii) each ankG is targeted by an antibody.

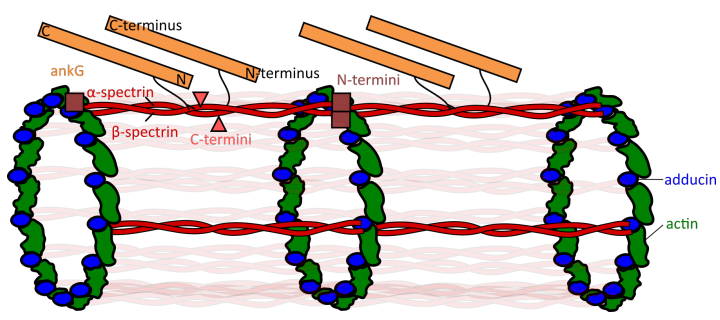

(a) Model of ankG in the AIS of an immature neuron.

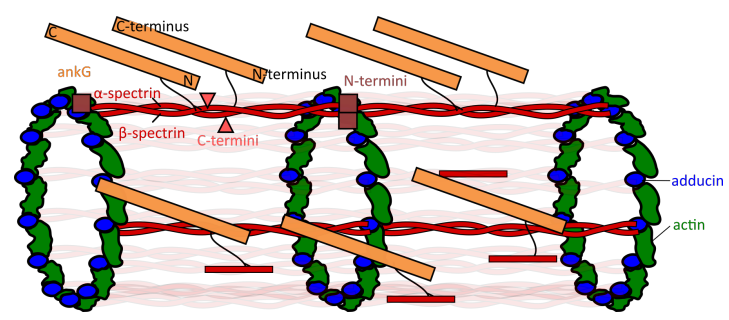

(b) Model of ankG in the AIS of a mature neuron.

Figure 39: Model of ankG organization in the AIS. (a) Immature neurons consist of $\alpha \mathrm{II} / \beta \mathrm{IV} \sum 1$-spectrin tetramers. AnkG has two organized binding positions at each $\alpha \mathrm{II} / \beta \operatorname{IV} \sum 1$-spectrin tetramer. (b) Mature neurons consist of $\alpha \mathrm{II} / \beta \mathrm{IV} \sum 1$-spectrin tetramers and the additional splice variant of spectrin, $\beta \operatorname{IV} \sum 6$, which is found as nonperiodically integrated. AnkG has two binding positions at each $\alpha \mathrm{II} / \beta \mathrm{IV} \sum 1$-spectrin tetramer and at least one binding position at each non-periodically integrated $\beta \operatorname{IV} \sum 6$ molecule.

\section{VGSC organization is (semi-)periodic.}

In VGSCs binding sites to ankG have been identified. ${ }^{68}$ The adapter protein ankG and the linker protein $\beta I V$-spectrin mediate the contact between the VGSCs and the underlying cytoskeleton (figure: 2). Here, a frequency of about $182 \mathrm{~nm}$ for VGSCs is identified. A similar organization has been lately found in neurons derived from rats. 11 Highly periodic and irregular areas are found (figure: 35) within a single AIS. This (semi-)periodic organization is not surprising, since VGSCs bind to ankG. As a consequence, a less pronounced periodic organization of ankG is transferred to the organization of VGSCs. VGSCs have an even (i) further distance to the underlying periodic network than ankG. Additionally, (ii) the adapter protein ankG has multiple binding positions on a single $\beta I V$-spectrin, which is likely to result in slight deviations in the distance between two ankG molecules and thus between VGSCs. Also, not all ankG molecules must be necessarily occupied by VGSCs. Further, (iii) the non-periodic organization of $\beta \operatorname{IV} \sum 6$ is likely to be transferred to VGSC organization. 
(Semi-)periodic $\mathrm{Na}_{\mathbf{v}} 1.6$ organization confirms findings obtained from VGSCs labeled by anti-panNav

So far, studies on subtypes have not been published. Here, a mean frequency of about $192 \mathrm{~nm}$ is found for $\mathrm{Na}_{\mathrm{v}} 1.6$ organization. This confirms the findings obtained within this thesis for the organization of all VGSC subtypes located in the AIS (results: 35 and fits to previously published data ${ }^{11}$ obtained in experiments with neurons derived from rats. Locally, the distance between two molecules is found to be higher than about $192 \mathrm{~nm}$ (high power at high frequencies in the power spectrum (figure: 35). The factors (i) to (iii), mentioned above for VGSCs detected by anti-pan $\mathrm{Na}_{\mathrm{v}}$, play also a role for $\mathrm{Na}_{\mathrm{v}} 1.6$ organization. Additionally, labeling and imaging artefacts cannot be excluded.

\section{$\mathrm{Na}_{\mathrm{v}} 1.2$ organization cannot be definitely determined.}

Since the subtypes $\mathrm{Na}_{\mathrm{v}} 1.6$ and $\mathrm{Na}_{\mathrm{v}} 1.2$ share a common binding motif (figure: 50 5 the same organization of the two subtypes is expected. The power spectra appear similar and a frequeny of about $187 \mathrm{~nm}$ was obtained by labeling for the subtype $\mathrm{Na}_{\mathrm{v}} 1.2$. This frequency is in the range under the consideration that the ankyrin-binding positions between on the spectrin molecules are closer than $200 \mathrm{~nm}$ to each other. Furthermore, the size of ankG molecules mediate a certain distance between the $\mathrm{Na}_{\mathrm{v}} 1.2$ channels and the periodic network. Nevertheless, the spectrum obtained for $\mathrm{Na}_{\mathrm{v}} 1.2$ labeling appears highly noisy. Especially, at high frequencies similar power values as at the frequency of $187 \mathrm{~nm}$ are found. An analysis cell-by-cell did not reveal more clear results. Thus, the data are not sufficient to subscribe or exclude a (semi-)periodic organization for $\mathrm{Na}_{\mathrm{v}} 1.2$. Biological and experimental causes are eligible to explain the uncertainty of the data. Biologically, (i) different expression or affinities of the two channel subtypes between DIV 11 to 20 cannot be excluded. If $\mathrm{Na}_{\mathrm{v}} 1.6$ expression is higher than $\mathrm{Na}_{\mathrm{v}} 1.2$ expression, $\mathrm{Na}_{\mathrm{v}} 1.6$ channels are more likely to occupy binding positions. Experimentally, (ii) localizations for $\mathrm{Na}_{\mathrm{v}} 1.2$ are likely to miss, due to failures of labeling and imaging. The labeling of VGSC subtypes was found to be challenging. For a final statement additional experiments are necessary (as discussed in 8). 
6. Axon inital SEgment MATURATION in HiPPOCAMPAL NEURONS DERIVED FROM $q v^{3 J}$

\section{Axon inital segment maturation in hippocampal neurons derived from $q v^{3 J}$ mutant mice}

Impaired information encoding in $q v^{3 J}$ mutant hippocampal neurons ${ }^{3}$, asked for an investigation of their VGSC density with maturation. Based on the certainty that the $q v^{3 J}$ mutation is a frameshift mutation in the cytoskeletal protein $\beta I V$-spectrin, abnormalities in AP waveforms are believed to be mediated by defects within the cytoskeletal component $\beta I V$-spectrin. Consequently, the expression pattern of $\beta I V$-spectrin and adjunct cytoskeletal components is monitored in control (wildtype and heterocygote) and $q v^{3 J}$ mutant hippocampal cultures (sibling cultures) over three weeks of maturation.

\subsection{Results}

$\beta I V$-spectrin, ankG and VGSCs are detected with a reduced mean fluorescence intensity in the AIS of $q v^{3 J}$ mutant neurons.

Results obtained from labeling control neurons for $\beta I V$-spectrin agree with previous experiments (figure: 18, 19). A significant AIS staining is observed over the three weeks of studied maturation using N-terminal and central labeling. $\beta I V$-spectrin is weakly, but significantly detected in the AIS of some immature $q v^{3 J}$ mutant neurons using N-terminal labeling (figure: 42 ). After the first week no neurons with a significant AIS staining are found. For central and C-terminal labeling no significant AIS staining is observed at any time point. The bright local somatic staining of the central antibody remains in $q v^{3 J}$ mutant neurons (figure: 58 .

$\mathrm{N}$ - and C-terminal labeling of ankG is significantly detected in the AIS of control and $q v^{3 J}$ mutant neurons (figure: 44). The mean fluorescence intensity in the AIS of $q v^{3 J}$ mutant neurons is lower as in control neurons.

VGSCs are found in the AIS of control and $q v^{3 J}$ mutant neurons. The number of $q v^{3 J}$ mutant neurons positive for VGSCs decreases with maturation. Meaning, whereby $92 \%$ show a significant VGSC staining at DIV 10, only $34 \%$ show a significant VGSC staining at DIV 19 (statistics: 40).

\footnotetext{
${ }^{3}$ Previous work: Electrophysiology measurements on control (wildtype/heterocygote) and $q v^{3 J}$ mutant neurons were performed by Elinor Lazarov, Hebrew University of Jerusalem, Israel during her PhD thesis. Manuscript: (2) Elinor Lazarov, Melanie Dannemeyer, Michael Gutnick, Fred Wolf, Andreas Neef "Reduced Nav channel density in the axon initial segment slows down the action potential onset and impairs high frequency encoding."
} 


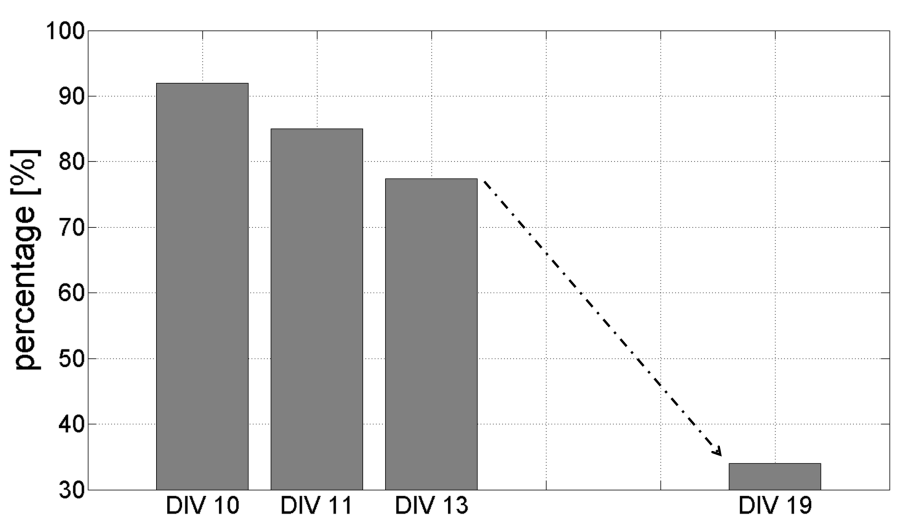

Figure 40: VGSC expression in $q v^{3 J}$ mutant neurons. Percentage of ankG positive AISs positive for VGSCs e.g. $92 \%$ at $D I V 10$ and $34 \%$ at $D I V 19 . \mathrm{N}=12,13,84,35$.

$\beta I V$-spectrin, ankG and VGSC: Mean fluorescence intensity does not increase with maturation. The proteins do not locally enrich in the AIS.

Profiles (figure: 43) extracted from control neurons labeled for $\beta I V$-spectrin N-terminal and central have the same behavior as previous obtained results from wildtype neurons (results: 34a. With maturation the mean fluorescence intensity increases. In the first and second week it increases strongly, whereas only a small increase from the second to the third week of studied maturation is observed. After the first week the intensity peaks at about $10-12 \mu \mathrm{m}$ from the soma or first branching point. In $q v^{3 J}$ mutants $\beta I V$-spectrin is not detected after the first week.

In control neurons, ankG labeled C-terminal shows no intensity increase over the three weeks of studied maturation. The mean fluorescence intensity peaks at about $10-12 \mu \mathrm{m}$. The mean fluorescence intensity of ankG labeled N-terminal shows an increase between the first and second week of studied maturation. Afterward, the mean fluorescence intensity stays constant. A peak is observed at about $10-12 \mu \mathrm{m}$. The results differ from previous once obtained in the wildtype study (results: 22). AnkG labeled C- and N-terminal in $q v^{3 J}$ mutant neurons has no significant mean fluorescence intensity increase in mature neurons. The profiles stay similar and appear relative smooth. A small intensity peak around $10-12 \mu \mathrm{m}$ is observed (figure: 45).

Immature neurons derived from control and $q v^{3 J}$ mutant mice show only a small difference of the mean fluorescence intensity using N- and C-terminal ankG labeling. Starting the second week ank $\mathrm{G}$ in control and $q v^{3 J}$ mutant neurons differs significantly. Whereas, ankG in control neurons is detected with a high fluorescence intensity, stays ankG labeled in $q v^{3 J}$ mutant neurons at a constant low fluorescence intensity.

Labeling against VGSCs in control neurons displays the same behavior as observed in previous experiments with wildtype neurons (results: 24). At the AIS, $q v^{3 J}$ mutant neurons have a lower mean fluorescence intensity from early on and do not reveal a peak. The profiles, obtained from 
6. Axon inital SEgment MATURATion in hippocampal NEURons DERIVEd From $q v^{3 J}$ 56

MUTANT MICE

VGSC positive neurons, show a constant mean fluorescence intensity with maturation.

\section{Somatic mean fluorescence intensity in the AIS of control and $q v^{3 J}$ mutant neurons does not differ.}

The mean fluorescence intensity in the AIS increases in control, but not in $q v^{3 J}$ mutant neurons with maturation. In $q v^{3 J}$ mutant neurons the mean fluorescence intensity stays at a low level. When looking at the mean fluorescence intensity of defined somatic regions in the same neurons the intensity is found to increase in control and $q v^{3 J}$ mutant neurons with maturation. Between the groups (e.g. at $D I V 9$ ) of control and $q v^{3 J}$ mutant neurons small, but non-significant differences are observed. A factor (method: 3.5.1 for presenting the relation between AIS and somatic mean fluorescence intensity was calculated. The mean fluorescence intensity in the AIS exceeds the somatic mean fluorescence intensity in control neurons 5 to 9 fold, whereas the exceed in mutant neurons is found at about 3 fold.

The number of $q v^{3 J}$ mutant neurons found $\mathrm{Na}_{\mathrm{v}} 1.2$ and $\mathrm{Na}_{\mathrm{v}} 1.6$ positive in the AIS is reduced.

Control neurons detected positive for ankG labeling are found positive for VGSCs, subtype $\mathrm{Na}_{\mathbf{v}} 1.2$ and subtype $\mathrm{Na}_{\mathrm{v}} 1.6$ at $D I V 14$ to 21 . Though, at $D I V 13$ only about $77.4 \%$ of $q v^{3 J}$ mutant neurons are found VGSC positive. At DIV 14 about $92 \%$ are found positive for subtype $\mathrm{Na}_{\mathrm{v}} 1.2$. Three days later, at $D I V 17$ only $14 \%$ are found $\mathrm{Na}_{\mathrm{v}} 1.6$ positive.

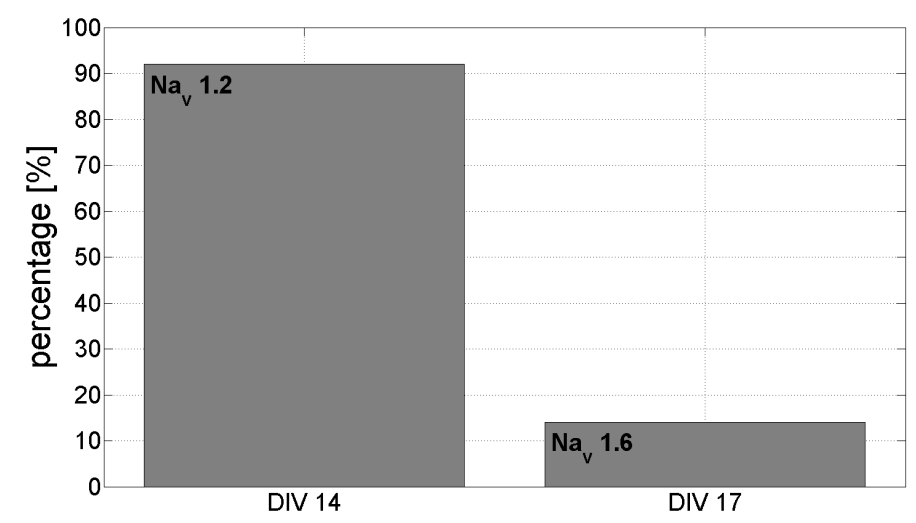

Figure 41: VGSC subtype expression in $q v^{3 J}$ mutant neurons. Percentage of ankG positive AISs positive for VGSC subtypes. DIV 14 neurons were chosen to be tested for $\mathrm{Na}_{\mathrm{v}} 1.2$ channels and $D I V 17$ for $\mathrm{Na}_{\mathrm{v}} 1.6$ channels based on results obtained from wildtype measurements, which demonstrate that DIV 14 neurons are highly positive for $\mathrm{Na}_{\mathrm{v}} 1.2$ channels (results $\mathrm{Na}_{\mathrm{v}} 1.2: 30$ 31) and older neurons are more likely positive for $\mathrm{Na}_{\mathrm{v}} 1.6$ channels (results $\mathrm{Na}_{\mathrm{v}} 1.6$ : 28 29). $92 \%$ of ankG positive $q v^{3 J}$ neurons were found positive for $\mathrm{Na}_{\mathrm{v}} 1.2 .14 \%$ of ankG positive $q v^{3 J}$ neurons were found positive for $\mathrm{Na}_{\mathrm{v}} 1.6 . \mathrm{N}=26,8$. 

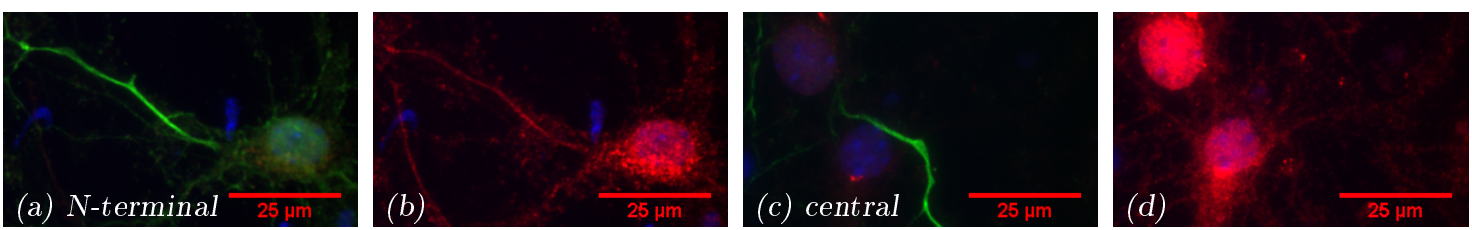

Figure 42: $\beta I V$-spectrin (red) labeled at $D I V \eta$ in $q v^{3 J}$ mutant. Double labeling with ankG at its N-terminus (green) in order to identify the AIS. Nucleus stained by DAPI (blue). (a) Merged images of $\beta I V$-spectrin labeled at its N-terminus and ankG. (b) BIV-spectrin channel only. Contrast adjusted in order to make AIS visible. The mean fluorescence intensity along the AIS is about 90 a.u. after the subtraction of dendritic fluorescence. (c) Merged images of $\beta I V$-spectrin labeled at a centrally located epitope and ankG. (d) $\beta I V$-spectrin channel only. Contrast adjusted in order to make potential labeled AIS visible. Scale bar: $25.0 \mu \mathrm{m}$.

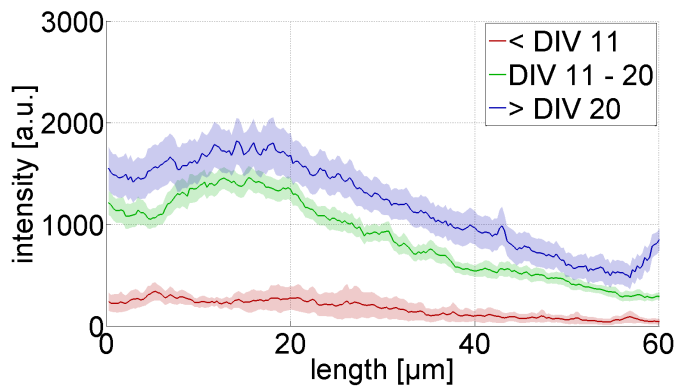

(a) Control: $\beta \mathrm{IV}$-spectrin labeled at its N-terminus

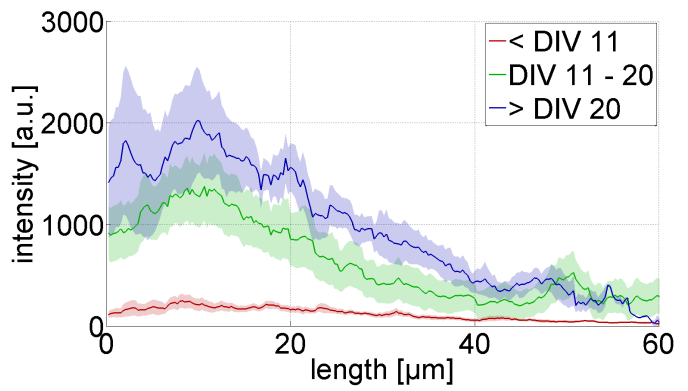

(b) Control: $\beta I V$-spectrin labeled at a central located epitope.

Figure 43: $\beta I V$-spectrin in development. Profiles show the mean fluorescence intensities [a.u.] along the AIS as a function of distance from the soma or first branching point at several time points within the first, second and third week in development. (a) $\beta I V$-spectrin N-terminal labeled. $\mathrm{N}=6,42,27$. (b) $\beta I V$-spectrin central labeled. $\mathrm{N}=11,20,9$. Error is the SEM. 
6. Axon inital SEgMENT MATURATION IN HiPPOCAMPAL NEURONS DERIVED FROM $q v^{3 J}$
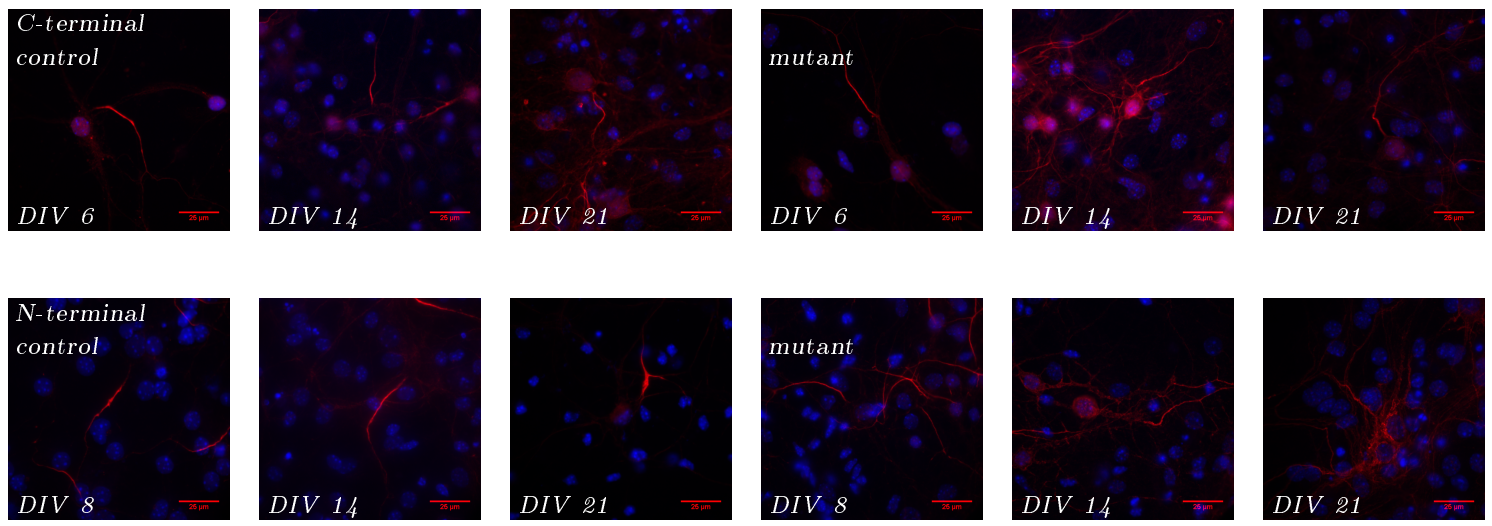

Figure 44: AnkG (red) labeled at its C-terminus (top row) (C-terminal antibody, sc-28561, 11.2 and N-terminus (bottom row) at DIV 6/8, DIV 14 and DIV 21 in control and $q v^{3 J}$ mutant neurons. Nucleus stained by DAPI (blue). Scale bar: $25 \mu \mathrm{m}$.

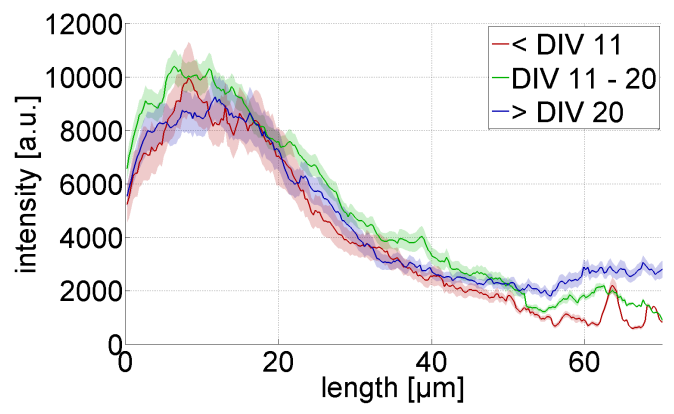

(a) Control: AnkG labeled at its C-terminus.

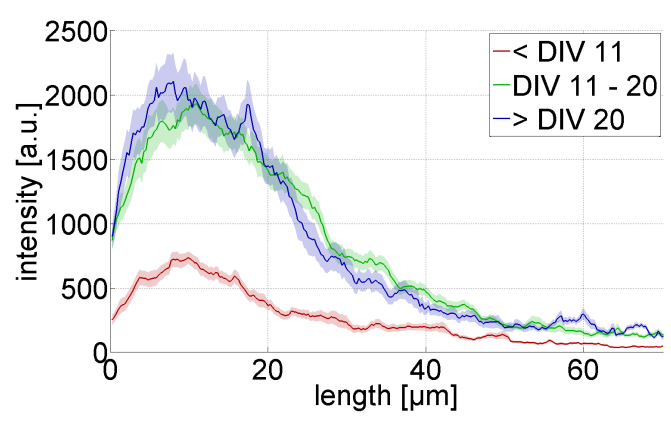

(c) Control: AnkG labeled at its N-terminus.

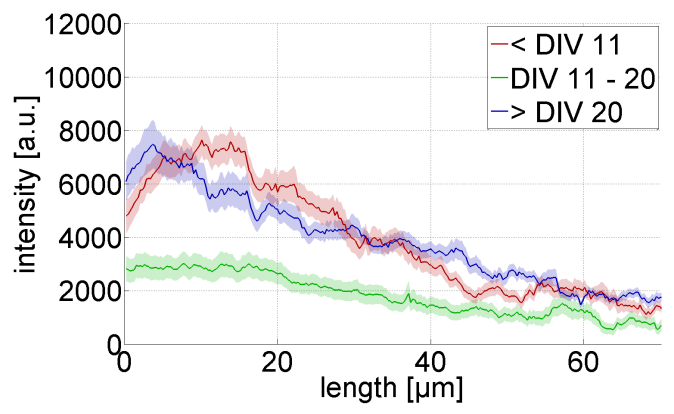

(b) Mutant: AnkG labeled at its C-terminus.

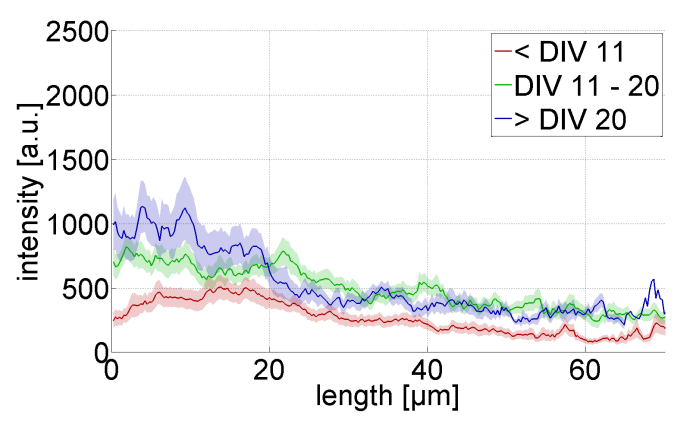

(d) Mutant: AnkG labeled at its N-terminus.

Figure 45: AnkG in development. Profiles show the mean fluorescence intensity along the AIS as a function of distance from the soma or first branching point at several time points within the first, second and third week in development. (a) Control C-terminal labeled (C-terminal antibody, sc-28561, 11.2 . $\mathrm{N}=38,69,53$. (b) $Q v^{3 J}$ mutant C-terminal labeled (C-terminal antibody, sc-28561, 11.2. $\mathrm{N}=35,56,44$. (c) Control N-terminal labeled. $\mathrm{N}=27,38,24$. (d) $Q v^{3 J}$ mutant N-terminal labeled. $\mathrm{N}=23,19,11$. Error is the SEM. 

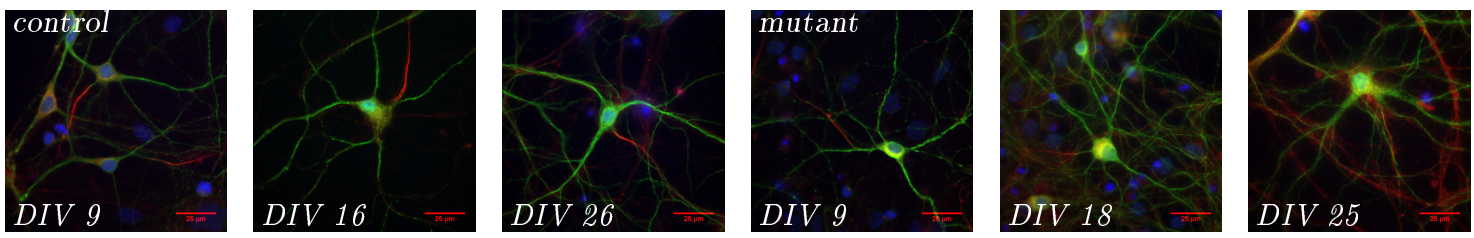

Figure 46: Voltage-gated sodium channels (red) at DIV 9, DIV 16/18 and DIV 25/26 in control and $q v^{3 J}$ mutant neurons. Double labeled with the marker MAP2 (green) at DIV 9, DIV 16/18 and DIV 25/26 highlights the AIS.

Nucleus stained by DAPI (blue). Scale bar: $25 \mu \mathrm{m}$.

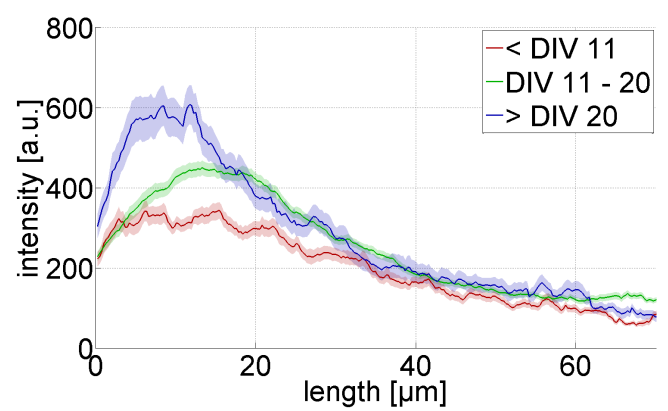

(a) Control: Mean fluorescence intensities [a.u.] along the AIS.

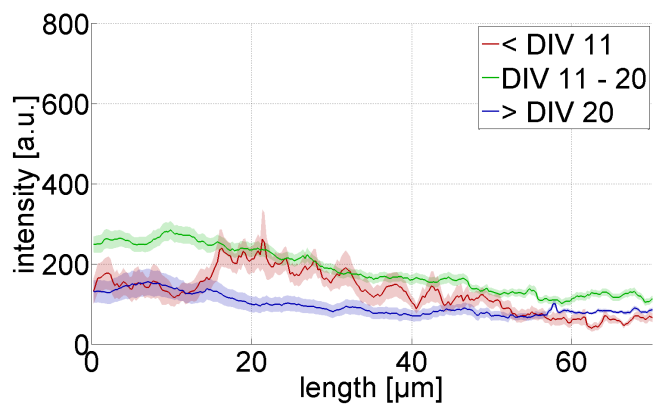

(b) Mutant: Mean fluorescence intensities [a.u.] along the AIS.

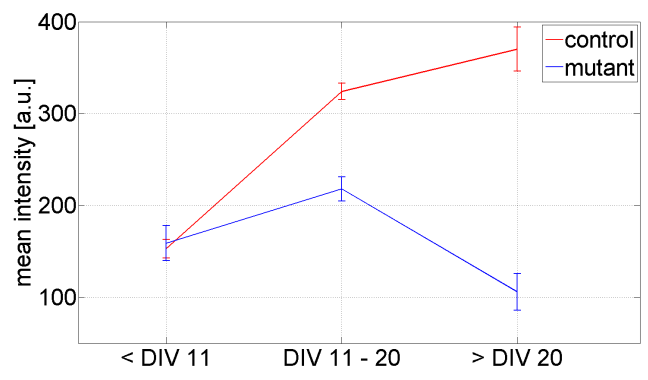

(c) Control and mutant: Mean fluorescence intensities

[a.u.] at the AIS within the distance $0-50.0 \mu \mathrm{m}$ from

the soma or first branching point.

Figure 47: Voltage-gated sodium channels in development. Profiles show the mean fluorescence intensites [a.u.] along the AIS as a function of distance from the soma or first branching point at several time points within the first, second and third week in development: (a) Control. $\mathrm{N}=56,260,67$. (b) VGSC positive $q v^{3 J}$ mutants. $\mathrm{N}$ $=9,88,33$. The profiles reveal a differential density and distribution of VGSCs in control and mutant neurons. (c) The graphs show the mean fluorescence intensities [a.u.] at the AIS within the distance $0-50.0 \mu \mathrm{m}$ from the soma or first branching point within the first, second and third week in development for control and $q v^{3 J}$ mutant neurons. The graphs reveal that VGSCs are differentially enriched along the AIS in control and mutant neurons. Within the first week the mean fluorescence intensity of anti-panNav labeling barely differs. After the first week, the mean fluorescence intensity increases for control and decreases for $q v^{3 J}$ mutant neurons. Error is the SEM. 
6. Axon inital SEgment MATURATion in hippocampal NEURons DERIVEd From $q v^{3 J}$ 60

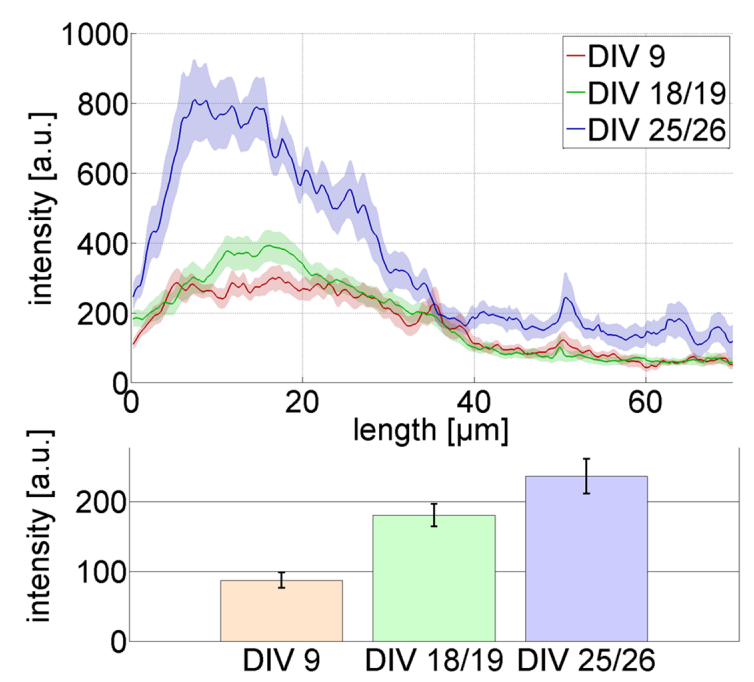

(a) Control neurons.

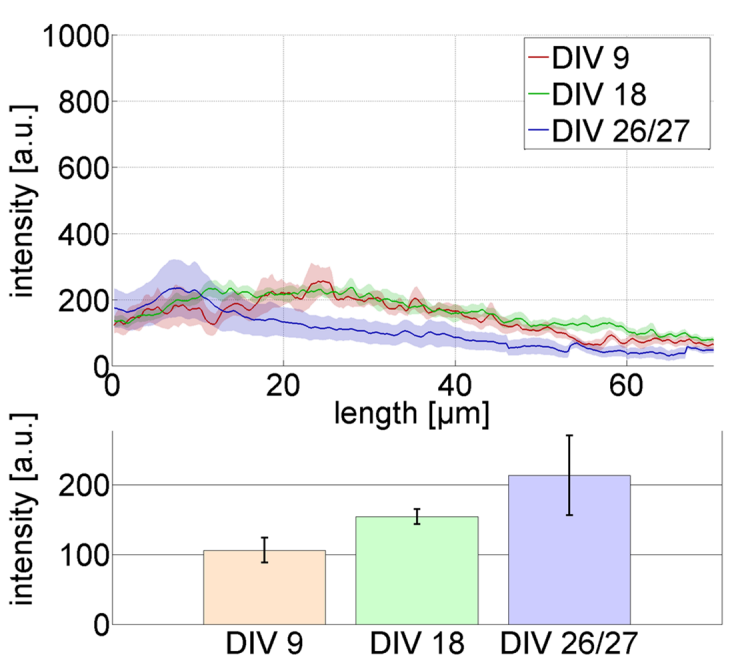

(b) $Q v^{3 J}$ mutant neurons.

Figure 48: Voltage gated sodium channels labeled by anti-panNa $\mathrm{a}_{\mathrm{v}}$ and analyzed cell-by-cell in the AIS and soma. (a) Control, $\mathrm{N}=13,26,11$ (b) $Q v^{3 J}$ mutant, $\mathrm{N}=7,10,8$. Control neurons are DIV 9, DIV 18/19 and DIV 25/26 and $q v^{3 J}$ mutant neurons are of similar age, DIV 9, DIV 18 and DIV 26/2\%. (Top) Profiles show the mean fluorescence intensities [a.u.] along the AIS as a function of distance from the soma or first branching point at three time points. (Bottom) The mean fluorescence intensities [a.u.] for defined somatic regions of the neurons are plotted as bars. The factor of the maximum mean intensity along the AIS versus mean intensity at the soma (factor = intensity (mean max. (AIS)/ mean (soma))) is for (a) control neurons 9.16 at DIV 9, 4.98 at DIV 18/19, and 5.55 at DIV 25/26 (b) $q v^{3 J}$ mutant neurons 3.0 at DIV 9,3.1 at DIV 18 and 3.2 at 26/27. Error is the SEM. 


\subsection{Discussion}

\section{Frameshift mutation $q v^{3 J}$ eliminates $\beta \operatorname{IV} \sum 1$ in early maturation and destabilizes the complete network.}

As previously described, and here confirmed by N-terminal and central labeling, the majority of $\beta \operatorname{IV} \sum 1$ is integrated in the cytoskeletal network from early on (discussion: 4.2). In some $q v^{3 J}$ mutant neurons a low density of $\beta I V$-spectrin is found in immature neurons. This is not surprising, since the mutation is located after the epitope targeted by the antibody. The relatively weak staining can be explained by an incomplete formation and destabilization of $\beta I V \sum 1$. Later on, destabilization results in a complete loss of $\beta I V$-spectrin. Destabilization of the $\beta I V$-spectrin network in mice lacking only $\beta \operatorname{IV} \sum 1$ has been described before. ${ }^{18}$ Also, a loss of $\beta$ IV-spectrin in $q v^{3 J}$ mutant neurons has been described before. $\frac{8[107}{107} \mathrm{~N}$-terminal and central antibodies target the same isoforms (figure: 56). An AIS labeling within the first week by central labeling is expected. This is not the case, e.g. due to different specificities of the antibodies. The staining observed in the somatic region next to the nucleus (figure: 58 remains in $q v^{3 J}$ mutant neurons (discussed: 9.1). The C-terminal antibody targets an epitope located after the frameshift mutation. As expected, no significant AIS labeling was observed from early on, confirming an improperly $\beta \operatorname{IV} \sum 1$ formation. Further, C-terminal labeling targets $\beta \operatorname{IV} \sum 6$, appearing later in maturation. Since no $\beta I V$-spectrin is detected in mature neurons, $\beta \operatorname{IV} \sum 6$ might be (i) improper formed, (ii) not stabilized, and (iii) not recruited, due to the lack of $\beta \operatorname{IV} \sum 1$. Again, this supports a later appearance of $\beta \operatorname{IV} \sum 6$ in the AIS.

Lack of $\beta I V$-spectrin results in a reduced ankG density. Recruitment of ankG is impaired. Remaining ankG is stabilized by $\beta I I-$ spectrin.

AnkG density in control and $q v^{3 J}$ mutant neurons has a small difference within the first week, but differs significantly in mature neurons. From these findings it can be concluded that ankG is affected by the $q v^{3 J}$ mutation. The small difference in immature neurons might be due to remaining $\beta I V$-spectrin. Afterward, $\beta I V$-spectrin stabilizes and possible recruits ankG in control neurons, whereas $q v^{3 J}$ mutant neurons lack the mechanism. Nevertheless, remaining ankG is stabilized in mature $q v^{3 J}$ mutant neurons (measurements were performed until DIV 24, figure: 73 , suggesting that the ankG stabilization depends not only on $\beta I V$-spectrin. Previously, $\beta I I-$ spectrin has been found to function as a binding partner for ankG, although it has no role in recruiting ankG and VGSCs. 127] 117 Its binding capability is likely stabilizing ankG in mature $q v^{3 J}$ mutant neurons. A rescue mechanism of $\beta \mathrm{IV}$-spectrin by $\beta \mathrm{II}$-spectrin, as described previously ${ }^{78}$, is excluded (results: 10.1. No increase of $\beta I I-$ spectrin (and ankG) density, while $\beta I V$-spectrin density decreases, is observed. This suggests its functionality as an exclusive stabilizing component. 
6. Axon inital SEgment MATURATION in HiPPOCAMPAL NEURONS DERIVED FROM $q v^{3 J}$

Previously, it has been published that ankG targeting and enrichment in $q v^{3 J}$ mutant neurons is non-affected. [1] [64 However, since no maturation studies have been performed and ankG is detected significantly in the AIS of mature neurons, an effect of the mutation on ankG might have been overseen within these studies.

Lack of $\beta I V$-spectrin destabilizes VGSCs in the AIS. Constant decrease of VGSCs, but not ankG, suggests a direct association with $\beta I V$-spectrin. AnkG is not sufficient for VGSC stabilization.

The obtained data for control neurons match with wildtype results (figure: 47 and 24. Within the first week VGSC density in the AIS of $q v^{3 J}$ mutant neurons as a total are not reduced, but lack differential regional enrichment in the AIS. Channels are likely attached by ankG to the remaining $\beta I V$-spectrin. With maturation the number of neurons positive for ankG, also positive for VGSCs, constantly decreases (results: 6.1). It is likely that the VGSCs cannot be stabilized throughout $q v^{3 J}$ mutant maturation. The constant ankG density (results: 6.1), suggests that VGSC stabilization depends not only on ankG binding, but requires an additional connection to the underlying cytoskeleton. Direct associations between $\beta$-spectrins and membrane proteins have been described. $[37 \lcm{128} \lcm{129}$

A constant increase of the VGSC density in the somatic region supports the hypothesis that destabilization and recruitment limitations of VGSCs are due to the effects of the $q v^{3 J}$ mutation on $\beta I V$-spectrin. Furthermore, abnormalities in VGSC expression can be excluded. This allows to conclude that the functionality of $\beta I V$-spectrin is important for maintaining high densities of VGSCs in the AIS.

\section{Lack of $\beta I V$-spectrin destabilizes $\mathrm{Na}_{\mathrm{v}} 1.2$ and prohibits $\mathrm{Na}_{\mathrm{v}} 1.6$ recruitment.}

At DIV $1492 \%$ of the AISs positive for ankG are found positive for $\mathrm{Na}_{\mathrm{v}} 1.2$. Consequently, $\mathrm{Na}_{\mathrm{v}} 1.2$ is found to be the main subtype in the AIS of $q v^{3 J}$ mutant neurons. At $D I V 17 \mathrm{Na}_{\mathrm{v}} 1.6$ is largely not detectable and thus likely absent. Since VGSC positive AISs were found to decrease in number, it is concluded that $\mathrm{Na}_{\mathrm{v}} 1.2$ channels cannot be stabilized in the AIS, due to a lack of $\beta I V$-spectrin, and $\mathrm{Na}_{\mathrm{v}} 1.6$ cannot be recruited in the AIS, due to a lack of the ankG- $\beta I V$-spectrin complex. These findings support the double role of $\beta I V$-spectrin as a stabilizer and recruiter. 


\section{Structural organization of axon initial segment components in hippocampal neurons derived from $q v^{3 J}$ mutant mice}

A structural study is necessary to understand the mechanism, which leads to the impaired information encoding 4 in $q v^{3 J}$ mutant neurons.

Two antibodies targeting $\beta I V$-spectrin at an epitope located before the frameshift mutation (figure: 56 were evaluated. Only one, the N-terminal $\beta I V-s p e c t r i n$ antibody, showed a weak AIS staining within the first week (results: 6.1 discussion: 6.2). Thus, N-terminal $\beta I V$-spectrin labeling was chosen to study the structure of $\beta I V$-spectrin in $q v^{3 J}$ mutant neurons.

AnkG was found to be expressed at a constant density, independently from the labeling position. Only N-terminal labeling revealed a periodic organization in wildtype neurons (results: 5.1. discussion: 5.2, although $\mathrm{N}$ - and C-terminal antibodies target the same splice variants. Consequently, the structure of ankG in $q v^{3 J}$ mutant neurons was studied using the N-terminal antibody.

AISs positive for VGSCs were found throughout the entire time of studied maturation (results: 6.1. A structural study between $D I V 11$ and 19 was performed.

Since ankG is constant expressed in $q v^{3 J}$ mutant neurons, it was found to be most suitable as an AIS marker. It was used for double labeling with anti- $\beta I V$-spectrin and anti-panNa $\mathrm{v}_{\mathrm{v}}$ in order to identify the AIS.

\subsection{Results}

$\beta I V$-spectrin is rare in the AIS of $q v^{3 J}$ mutant neurons. Remaining $\beta I V$-spectrin lacks periodic organization.

Sibling control and $q v^{3 J}$ mutant derived neurons were imaged (figure: 50 . Control neurons show a periodicity of about $196 \mathrm{~nm}$, comparable to $190 \mathrm{~nm}$ observed in wildtype neurons. As described before (results: 6.1), most $q v^{3 J}$ mutant neurons are negative for $\beta I V$-spectrin. Below DIV 10 some AISs are found weakly positive for $\beta I V$-spectrin (figure: 50, example at DIV 7 ). Localizations within $\beta I V$-spectrin positive $q v^{3 J}$ mutant neurons are rare. The extracted example profile displays only three localizations (figure: 50. A noisy power spectrum without an outstanding power at any frequency is obtained.

\footnotetext{
${ }^{4}$ Previous work: Electrophysiology measurements on control (wildtype/heterocygote) and $q v^{3 J}$ mutant neurons were performed by Elinor Lazarov, Hebrew University of Jerusalem, Israel during her PhD thesis. Manuscript: (2) Elinor Lazarov, Melanie Dannemeyer, Michael Gutnick, Fred Wolf, Andreas Neef "Reduced Nav channel density in the axon initial segment slows down the action potential onset and impairs high frequency encoding."
} 


\section{AnkG in $q v^{3 J}$ mutant neurons is highly regularly organized.}

AnkG labeled N-terminal in control neurons is comparable with previously obtained results (results: 5.1. An even more prominent periodic pattern is observed in the AIS of $q v^{3 J}$ mutant neurons.

Example profiles of $1.0 \mu \mathrm{m}$ segments (profiles: 51a) were extracted. At DIV 11 the profile of a $q v^{3 J}$ mutant neuron shows five defined localizations. The control neuron does not show defined localizations. Overlapping localizations cannot be explicit distinguished. At DIV 13/14 and 19 a similiar behavior is found. Whereas the $q v^{3 J}$ mutant neurons display clearly defined localizations, appear the control neurons less structured.

The extracted power spectrum from control neurons at $D I V 11$ reveals a small, broad peak at the spatial frequency of about 190 to $200 \mathrm{~nm}$. At higher frequencies the spectrum is noisy. The $q v^{3 J}$ mutant does not have a distinct power maximum. At least two maxima between the spatial frequencies of 190 and $200 \mathrm{~nm}$ are found. At DIV 13/14 both power spectra appear less noisy. For $q v^{3 J}$ mutant neurons a distinct peak at a frequency of about $192 \mathrm{~nm}$ is observed. For control neurons the peak appears less defined and has multiple shoulders. Additionally, especially the spectrum of the control neurons appears noisy at high spatial frequencies. At $D I V 19$, control and $q v^{3 J}$ mutant neurons appear with a distinct power maximum at the spatial frequencies of about 190 to $200 \mathrm{~nm}$.

\section{Organization of remaining VGSCs in $q v^{3 J}$ mutant neurons is maintained.}

Neurons were labeled for ankG C-terminal (C-terminal antibody, sc-28561, 11.2 and VGSCs. Neurons were chosen by being ankG positive. All AISs of control neurons positive for ankG are positive for VGSCs. For $q v^{3 J}$ mutant neurons two subgroups of ankG positive neurons are found: (i) positive and (ii) negative for VGSCs.
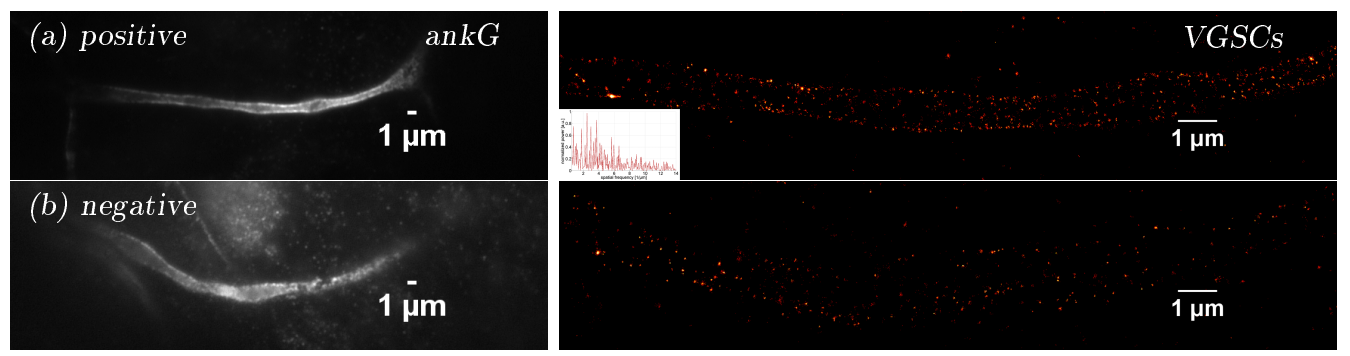

Figure 49: Sodium channels in $q v^{3 J}$ mutant neurons. Example images at DIV 19. (a) AnkG (wide-field image, grey) positive AIS is VGSC (STORM image) positive. Corresponding power spectrum is shown. (b) AnkG (widefield image, grey) positive AIS is VGSC (STORM image) negative.

The number of ankG positive AISs of $q v^{3 J}$ mutant neurons, positive for VGSCs decreases with 
7. Structural ORganization of aXon initial SEGMENT COMPONENTS IN HiPPOCAMPAL NEURONS DERIVED FROM $q v^{3 J}$ MUTANT MICE

maturation (statistics: 40.

$Q v^{3 J}$ mutant neurons, which are highly positive for VGSCs, show a clear dense appearance of localizations in the STORM image and are undistinguishable from control neurons (figure: 49 (a)). $Q v^{3 J}$ mutant neurons with AISs negative for VGSCs show no or a very low amount of localizations in the STORM image. The number of localizations is not sufficient for identifying a structure (figure: 49 (b)).

From VGSC positive AISs of $q v^{3 J}$ mutant and control neurons the power spectra are calculated. At $D I V 10 / 11$ control and $q v^{3 J}$ mutant neurons have a peak at a spatial frequency of about $200 \mathrm{~nm}$. Comparable power values are found at higher frequencies. At DIV 13/14 the control has a high power peak at about $200 \mathrm{~nm}$. Again similar power values are observed at higher frequencies. The $q v^{3 J}$ mutant neurons indicate a small power peak at $200 \mathrm{~nm}$, which is covered by high power values at higher frequencies. At $D I V 19$ a distinct power peak at about $200 \mathrm{~nm}$ for the control neurons is observed, but the mean spectrum of $q v^{3 J}$ mutant neurons appears strongly noisy. 
7. Structural ORganization of AXON initial SEgment COMPONENTS IN Hippocampal
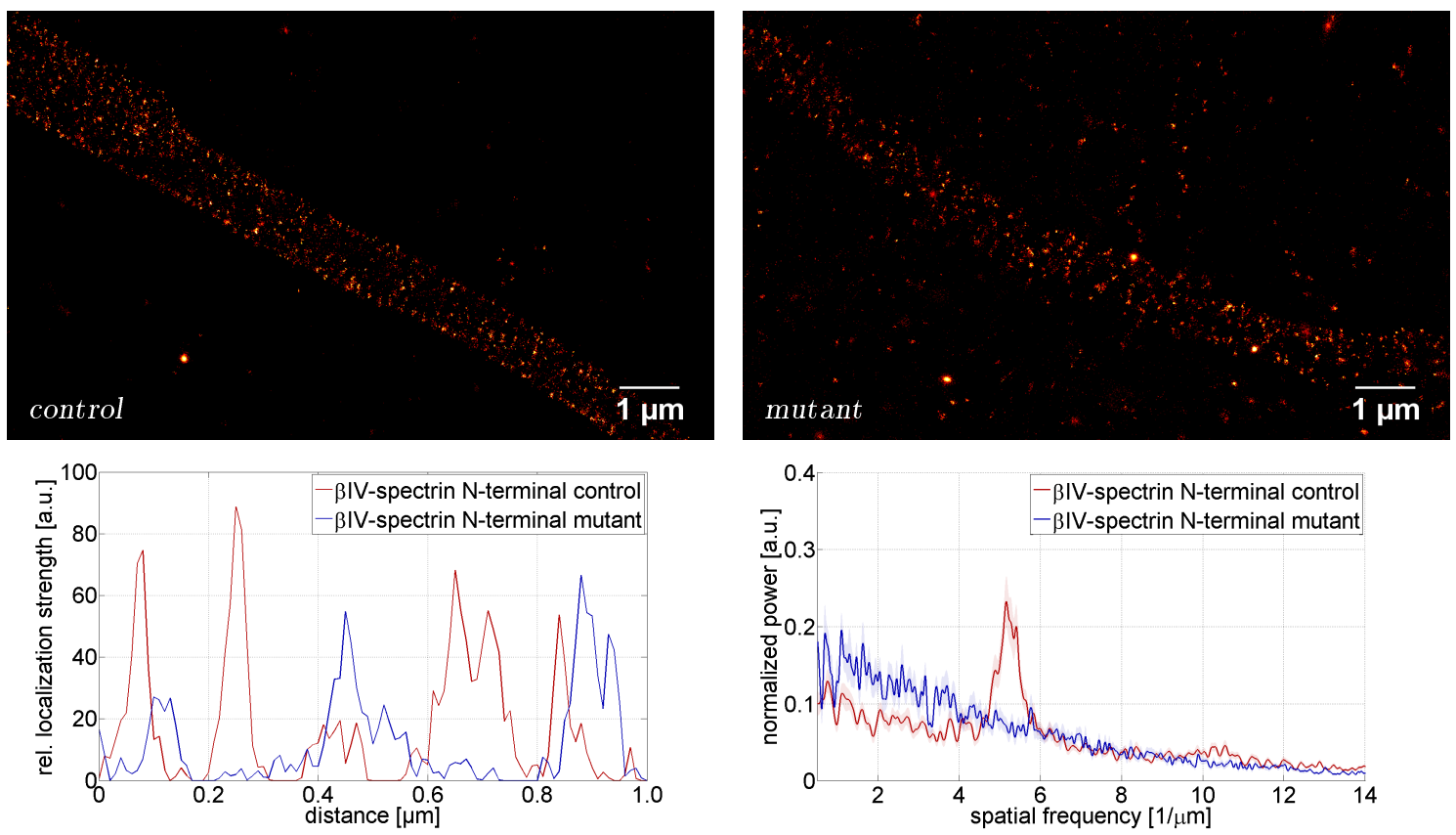

(a) Profiles of $1.0 \mu \mathrm{m}$ segments extracted from the shown (b) Power spectra calculated from the complete $\beta I V-$ neurons. spectrin data set.

Figure 50: Structural comparison of $\beta I V$-spectrin in control and $q v^{3 J}$ mutant neurons at the age $<D I V 11$ by STORM imaging. (a) Profiles of $1.0 \mu \mathrm{m}$ segments extracted from the shown neurons show regular localizations for control and sparse localizations for $q v^{3 J}$ mutant neurons. (b) Power spectra calculated from the complete $\beta I V-$ spectrin data sets reveal a periodic organization of about $196 \mathrm{~nm}$ for control neurons and no defined structure for $q v^{3 J}$ mutant neurons. $\mathrm{N}=52,32$. Error is the SEM. 
7. Structural organization of AXON Initial SEGMENT COMPONENTS IN HiPPOCAMPAL NEURONS DERIVED FROM $q v^{3 J}$ MUTANT MICE
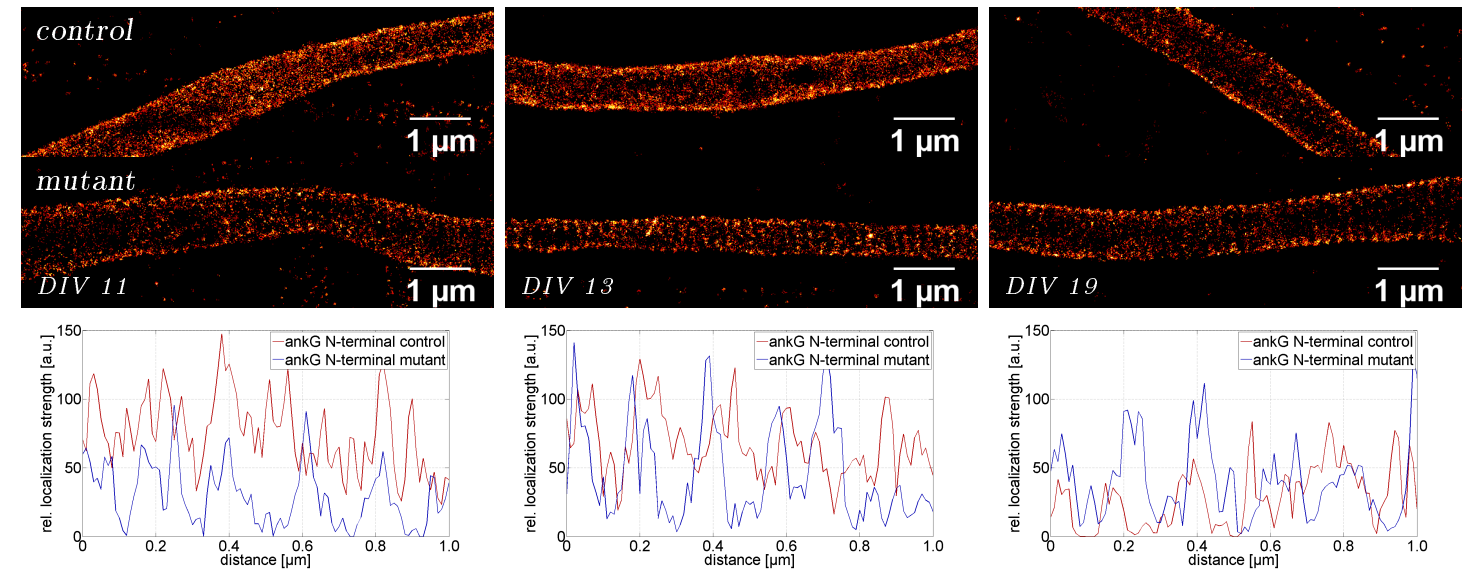

(a) $D I V 11$

(b) DIV 13/14

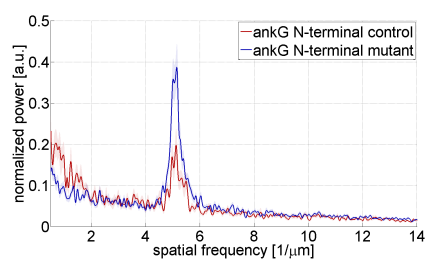

(e) DIV 13/14 (c) $D I V 19$

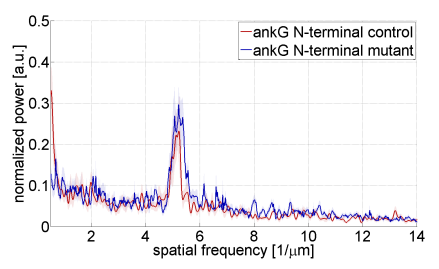

(f) $D I V 19$

Figure 51: Structural comparison of ankG in control and $q v^{3 J}$ mutant neurons at DIV 10/11, 13/14 and 19 by STORM imaging. (a - c) Profiles of $1.0 \mu \mathrm{m}$ segments extracted from the shown neurons, show overlapping localizations for control neurons and defined single localizations for $q v^{3 J}$ mutant neurons. (d - f) Power spectra calculated from the complete ankG data sets for control and $q v^{3 J}$ mutant neurons at DIV 10/11, 13/14 and 19 reveal a periodic organization of about 190 to $200 \mathrm{~nm}$. $\mathrm{N}($ control $)=17,28,14 ; \mathrm{N}$ (mutant) $=9,53,22$. Error is the SEM. 


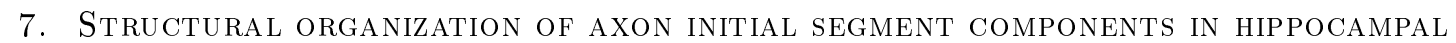
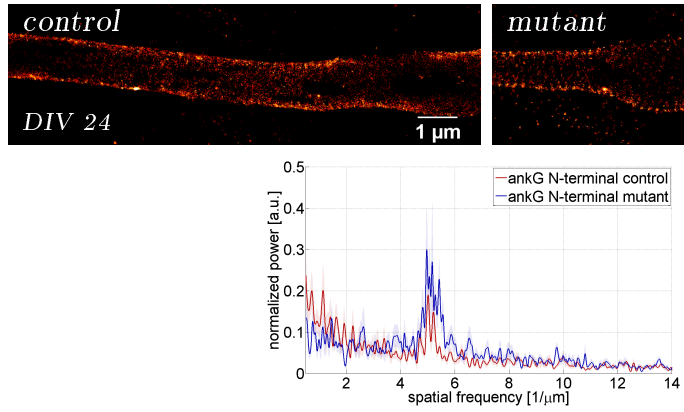

(a) Power spectra calculated from the complete ankG data sets

Figure 52: Structural comparison of ankG in control and $q v^{3 J}$ mutant neurons by STORM imaging at DIV 24 in order to investigate, if the effect of the $q v^{3 J}$ mutation on ankG proceeds with maturation. (a) Power spectra calculated from the complete ankG data sets for control and $q v^{3 J}$ mutant neurons at DIV 24 reveal a pronounced periodic organization of about $200 \mathrm{~nm}$ for $q v^{3 J}$ mutant neurons and a less pronounced organization for control neurons. AnkG periodicity is maintained throughout the fourth week in $q v^{3 J}$ mutant neurons. $\mathrm{N}=12,10$. Error is the SEM. 
7. Structural organization of AXON Initial SEgMENT COMPONENTS IN HiPPOCAMPAL NEURONS DERIVED FROM $q v^{3 J}$ MUTANT MICE

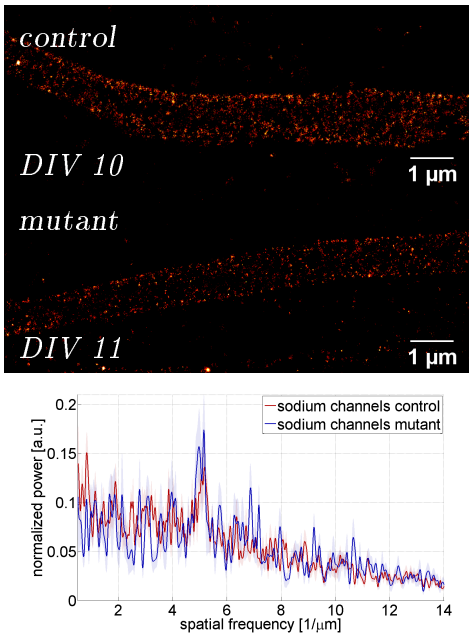

(a) $D I V 10 / 11$
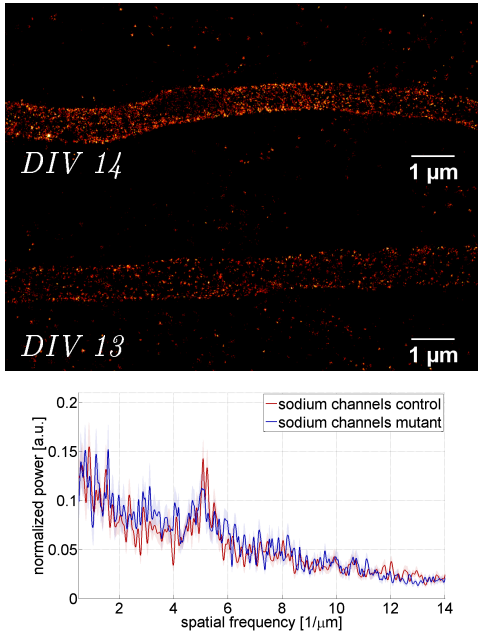

(b) $D I V 13 / 14$
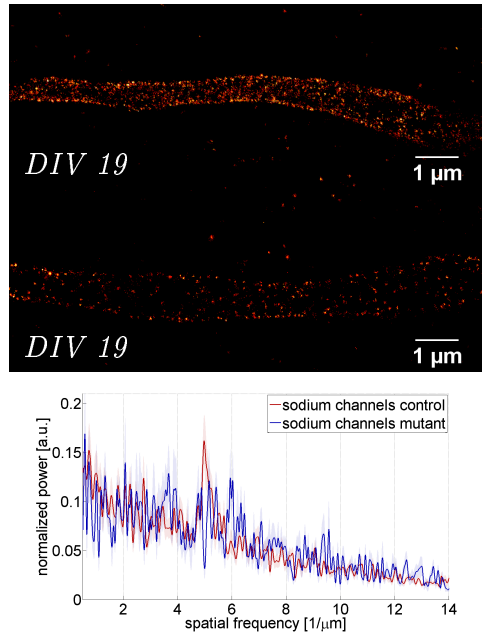

(c) DIV 19

Figure 53: Structural comparison of VGSCs in control and $q v^{3 J}$ mutant neurons at DIV 10/11, 13/14 and 19 by STORM imaging. ( $\mathrm{a}-\mathrm{c}$ ) Power spectra calculated from the complete VGSC data sets for control and $q v^{3 J}$ mutant neurons at $D I V 10 / 11,13 / 14$ and 19 reveal a (semi-)periodic organization of about $200 \mathrm{~nm}$. $\mathrm{N}($ control) $=20,33$, 33. $\mathrm{N}$ (mutant $)=10,29,12$. Error is the SEM. 


\subsection{Discussion}

$\beta I V-s p e c t r i n$ lacks its periodic organization from early on, suggesting frameshift $q v^{3 J}$ mutation is severe.

$\beta I V$-spectrin is highly affected by the frameshift $q v^{3 J}$ mutation. 118 A weak staining within the first week, using an N-terminal antibody, is detectable. The structural imaging of the remaining $\beta I V$-spectrin, does not reveal an organized network (figure: 50 ).

The actin-binding domain is located before the frameshift $q v^{3 J}$ mutation. Consequently, the binding of $\beta I V$-spectrin (specifically $\beta \operatorname{IV} \sum 1$ ) to the underlying periodic actin-adducin ring cytoskeleton could be unaffected. For spectrin tetramer formation an interaction with the $\beta$ spectrin repeat 17 takes place. 59 [16] [19] The repeat is located behind the frameshift mutation and likely to be incomplete. Consequently, no complete spectrin tetramers can be formed, resulting in an incomplete periodic organization and insufficient network stabilization.

AnkG is stabilized by $\beta I V$ - and $\beta I I-s p e c t r i n:$ A periodic $\beta I V$-spectrin isoform $\left(\beta I V \sum 1\right)$ within the network mediates periodic ankG placement. A non-periodic $\beta I V$-spectrin isoform $\left(\beta \operatorname{IV} \sum 6\right)$ within the network mediates non-periodic ankG placement. $\beta I I-$ spectrin is an additional binding partner for ankG and mediates periodic ankG organization.

In control neurons (figure: $34,5.2$ ankG appears organized, but does not have a striking periodic pattern as observed for $\beta I V$-spectrin labeled N-terminal (figure: 32]. This was explained by the circumstance that ankG is not a part of the underlying cytoskeletal ring-structured network and periodic organization is only mediated by binding to the structure (details: 5.2). AnkG is affected by the $\beta I V$-spectrin frameshift mutation, but is found at a constant density. Surprisingly, remaining ankG is found more prominently periodically organized in the $q v^{3 J}$ mutant, than in control neurons. Since $\beta I V$-spectrin is gone after the first week in $q v^{3 J}$ derived neurons, it is concluded that ankG has another additional binding partner (discussion: 6.2). Here $\beta I I-$ spectrin is suggested. III-spectrin has been believed earlier to bind, but not recruit, ankG [127 [62] [17] and thus is a candidate as an ankG stabilizer within the network. This is likely, since $\beta$ II-spectrin is, identically to $\beta \operatorname{IV} \sum 1$, periodically organized.

Concluding, in $q v^{3 J}$ mutant neurons ankG binds in immature neurons to $\beta I V \sum 1$ and $\beta I I-$ spectrin. In mature $q v^{3 J}$ mutant neurons ankG binds only to $\beta I I-$ spectrin. Also, no additional ankG molecules can be recruited. As a consequence, ankG density does not increase. In control neurons, ankG binds to $\beta \operatorname{IV} \sum 1, \beta I I-s p e c t r i n$ and starting within the second week to the nonperiodic $\beta \operatorname{IV} \sum 6$. Additional ankG molecules can be recruited and the density increases. Thus, a partly non-periodic ankG organization is found in control and a complete periodic organization in 
$q v^{3 J}$ mutant neurons (figure: 54 .

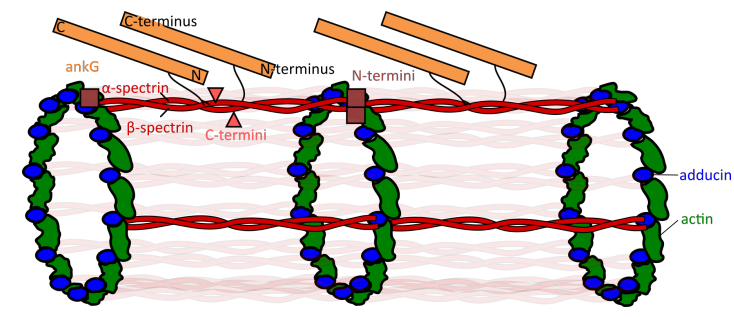

(a) AnkG organization in mature $q v^{3 J}$ mutant neurons.

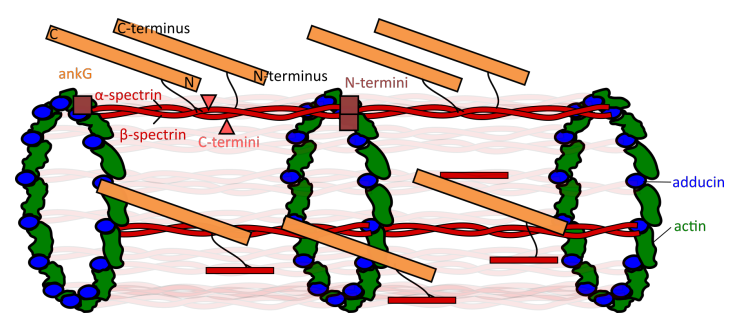

(b) AnkG organization in mature control neurons.

Figure 54: Potential ankG binding partners. (a) In mature $q v^{3 J}$ mutant neurons $\beta I V$-spectrin is lost. $\alpha \mathrm{II} / \beta \mathrm{II}-$ spectrin tetramers remain, are periodically organized and are binding partners for ankG. The model shows ankG bound to $\alpha \mathrm{II} / \beta \mathrm{II}$-spectrin tetamers with the spectrin-binding domain located about one fourth from the N-terminal end of the ankG sequence. (b) In the control neurons $\alpha \mathrm{II} / \beta \mathrm{IV}$-spectrin is found in two splice variants. $\alpha \mathrm{II} / \beta \mathrm{IV} \sum 1$ spectrin tetramers are periodically organized and besides $\alpha \mathrm{II} / \beta \mathrm{II}$-spectrin tetamers a periodically organized binding partner for ankG. The non-periodically organized $\beta \operatorname{IV} \sum 6$, which lacks an actin-binding domain, disrupts the clear organization of ankG molecules in controls.

(Semi-)periodic VGSC organization remains in VGSC positive $q v^{3 J}$ mutant neurons. At $D I V 11$ the control and $q v^{3 J}$ mutant neurons have a comparable VGSC organization with a mainly $200 \mathrm{~nm}$ spacing. Additionally, areas with higher frequencies and thus sparser VGSC density are found. Consequently, the VGSCs appear (semi-)periodic (previous findings: [11]). It has to be considered that VGSCs are not fully expressed at $D I V$ 11, which explains higher distances between VGSC localizations (results: 24 previous findings:[12]).

In control neurons no changes between $D I V 11$ and $13 / 14$ are observed. In $q v^{3 J}$ mutant neurons the organization is less pronounced. At $D I V 19$ control neurons have an even more prominent (semi-)periodic organization, than at $D I V$ 13/14. The $q v^{3 J}$ mutant neurons have a low level of organization, due to the low number of remaining VGSCs. The findings support the hypothesis that remaining VGSCs do not lose their organization. Simply, their density decrease leads to a higher distance between the VGSCs. To conclude, the effect of the $q v^{3 J}$ mutation on the VGSC stabilization in mature neurons is protruding. Since $\beta I V$-spectrin lacks as a mediator between periodically organized actin-adducin rings and VGSCs, another component, likely $\beta$ II-spectrin, is the linker between the cytoskeleton and VGSCs. 


\section{Conclusion}

AIS maturation and structural constitution is still not fully understood. Previous studies are diverse and models kept simple. Cytoskeletal proteins, actin, adducin and spectrin are believed to constitute to a periodic network. Within this model the proteins actin and adducin form rings, which are connected by spectrins. The adapter protein ankG has multiple binding positions on the spectrin molecules and functions as an adapter for membrane associated and integral proteins. Thus, a (semi-)periodic organization is transferred to membrane proteins, such as VGSCs. 11

Within this thesis, the basic constitution of the structural AIS model is confirmed.11 Detailed studies on $\beta I V$-spectrin isoforms support that it is important for stabilization and recruitment of AIS proteins. Not all isoforms are build in a periodic matter in the network. $\beta I V \sum 1$ has an actinbinding domain $\left[\frac{18}{18}\right.$ and is found to form spectrin tetramers connecting actin-adducin rings. It is fully expressed early, stabilizes the cytoskeletal network and recruits AIS proteins. Experiments with $q v^{3 J}$ mutant neurons showed that a functional C-terminus is crucial for stabilization and recruitment of AIS proteins. $\beta \operatorname{IV} \sum 6$ lacks an actin-binding domain $[126$, its organization does not base on the actin organization, which leads to an irregular appearance. It is expressed largely starting the second week. At this time point it is believed to take over a major role in VGSC recruitment and local enrichment in the AIS. Especially the enrichment of $\mathrm{Na}_{\mathrm{v}} 1.6$ channels, which density increases tremendously between the second and third week of maturation, is likely linked to $\beta I V \sum 6$ expression.

The incomplete periodic $\beta I V$-spectrin organization is transferred on $\beta I V$-spectrin binding proteins, such as ankG, and thus on their binding partners, the membrane proteins. Previously, ankG has been described as periodic, but with less prominence, due to multiple close located binding positions on each spectrin tetramer. ${ }^{12}$ This might play a role, however its binding to the non-periodically organized $\beta \operatorname{IV} \sum 6$, which increases in density with maturation, leads to an increase of the density of non-periodical organized ankG.

Now that it is known that $\beta I V$-spectrin isoforms underlie different organization, expression and have different roles, the question arisis if ank $\mathrm{G}$ isoforms appear in a similar way. First of all the lack of $\beta I V$-spectrin, but the constant ankG density suggests an additional binding partner for ankG. Further, ankG in mice lacking $\beta I V$-spectrin appears more pronounced periodic. Together these findings suggest that ankG binds to another periodically organized structure than $\beta I V$-spectrin. The constant ankG density suggests that this binding partner stabilizes, but does not recruit ankG. Here it is concluded that $\beta$ II-spectrin is a stabilizer of ankG. It has been earlier suggested to bind, 
but not recruit ankG. $[127 \lcm{62[17}$

Recently, a new study on ankG binding has been published. ${ }^{73}$ A mutation in the spectrinbinding domain (www.uniprot.org, Q12955) of giant $480 \mathrm{kDa}$ ankG showed no effect on $\beta I V-$ spectrin recruitment and enrichment. ${ }^{[73}$ This suggests that giant $480 \mathrm{kDa}$ ankG recruits and locally stabilizes $\beta I V$-spectrin with another binding motif. Experiments revealed a serine, located at amino acid region 1900. The 1900 amino acid region is missing in the $270 \mathrm{kDa}$ ankG splice form. Their experiments support that this isoform does not recruit $\beta I V$-spectrin. Consequently, the spectrin-binding domain ( $\mathrm{ZU}$ 5) in giant $480 \mathrm{kDa}$ and $270 \mathrm{kDa}$ ankG does not function as a recruitment domain for $\beta I V$-spectrin. 73

In future work it would be interesting to investigate if differential binding mechanisms of ankG isoforms are present in the AIS. Implying, if both ankG isoforms bind to $\beta I V-$ spectrin and $\beta I I-$ spectrin. This aspect can be investigated by ankG isoform specific labeling in control and $\beta I V-$ spectrin lacking $q v^{3 J}$ mutant neurons. Simple wide-field microscopy allows to determine if both isoforms are present in $q v^{3 J}$ mutant neurons. If giant $480 \mathrm{kDa}$ and $270 \mathrm{kDa}$ ankG bind to $\beta \mathrm{II}-$ spectrin, both are expected to be detected in $q v^{3 J}$ mutant neurons. The binding of both isoforms to $\beta I I-s p e c t r i n$ is likely, since both have a spectrin-binding domain. Giant $480 \mathrm{kDa}$ ankG has been shown not to recruit $\beta I V$-spectrin by the spectrin-binding domain, but a serine motif, consequently the spectrin-binding domain is even more likely to function as a binding motif for ankG stabilization by $\beta I I-$ spectrin. $270 \mathrm{kDa}$ ankG has been shown to have no serine motif and failed to recruit $\beta I V-$ spectrin in the AIS ${ }^{[73}$, thus it is likely that also $270 \mathrm{kDa}$ ankG binds to $\beta I I-s p e c t r i n$, rather than $\beta I V$-spectrin, by the spectrin-binding domain. This leads to the hypothesis that $\beta I V$-spectrin is only found to bind giant $480 \mathrm{kDa}$ ankG. Thereby a reduced, but constant density of giant $480 \mathrm{kDa}$ ankG is expected to be found in $q v^{3 J}$ mutant neurons. Otherwise a similar density of $270 \mathrm{kDa}$ ankG is expected to be detected in wildtype and $q v^{3 J}$ mutant neurons. Further, STORM imaging is suitable to reveal structural differences. If $270 \mathrm{kDa}$ ankG binds only to $\beta I I-s p e c t r i n$ it is expected to be prominently periodically organized in the AIS at all ages, including in mature neurons. On the other hand, binding to $\beta I V$-spectrin should display a decrease of periodic organization with maturation. In this case giant $480 \mathrm{kDa}$ ankG should display a decrease of periodic organization with an increase of the $\beta \operatorname{IV} \sum 6$ density.

The density of $\beta$ II-spectrin was measured similarly in wildtype and $q v^{3 J}$ mutant neurons. This allows to conclude that $\beta$ II-spectrin plays a role in the cytoskeletal stabilization of the AIS, but does not rescue for $\beta I V$-spectrin absence. ${ }^{78}$ Additionally, the constant expression of ankG supports that $\beta I I-s p e c t r i n$ does not take over the recruitment function of $\beta I V$-spectrin. Thus rescue mechanims between $\beta$-spectrins cannot be supported by these findings. 
(Semi-)periodic organization has been described for VGSCs. [11 The (semi-)periodic, but not pronounced periodic organization was explained by lacking a direct binding to the underlying periodic cytoskeleton, but binding to the irregularly spaced ankG molecules. Again, the nonperiodically organized $\beta \operatorname{IV} \sum 6$ needs to be taken into account. A differential organization of subtype $\mathrm{Na}_{\mathrm{v}} 1.2$ and $\mathrm{Na}_{\mathrm{v}} 1.6$ has not been questioned. Within this thesis, a comparable organization of sodium channels labeled by anti-panNa $\mathrm{Na}_{\mathrm{v}}$ and subtype $\mathrm{Na}_{\mathrm{v}} 1.6$ was revealed, whereas $\mathrm{Na}_{\mathrm{v}} 1.2$ organization could not be definitely revealed, although, $\mathrm{Na}_{\mathrm{v}} 1.2$ and $\mathrm{Na}_{\mathrm{v}} 1.6$ share a common ankG binding motif. ${ }^{68}$. It needs to be mentioned that subtype specific labeling has been found challenging (results: 9.1.4, 9.1.3. For future work it would be of interest to prove differential VGSC organization by subtype specific knockout mice. This implies that labeling of VGSCs in $\mathrm{Na}_{\mathrm{v}} 1.6$ and $\mathrm{Na}_{\mathrm{v}} 1.2$ knockout mice is performed by the same anti-panNa antibody. If $\mathrm{Na}_{\mathrm{v}} 1.2$ and $\mathrm{Na}_{\mathrm{v}} 1.6$ are organized in the same way, then the structure revealed by anti-panNa $\mathrm{Nabeling}_{\mathrm{v}}$ in $\mathrm{Na}_{\mathrm{v}} 1.2$ and $\mathrm{Na}_{\mathrm{v}} 1.6$ knockout mice is expected to be identical. In contrast to subtype specific labeling, the usage of the same antibody ensures that antibody properties, such as different affinities and low labeling success, do not influence the results.

Experiments with $\beta I V$-spectrin lacking neurons show a decrease of VGSC density at a constant ankG density. This suggests that ankG is not the only connection between channels and the underlying cytoskeleton. Since direct interactions of spectrins and membrane proteins have been described before, a direct interaction of VGSCs with $\beta I V$-spectrin has to be taken into account. Looking specifically at the subtypes $\mathrm{Na}_{\mathrm{v}} 1.2$ and $\mathrm{Na}_{\mathrm{v}} 1.6, \mathrm{Na}_{\mathrm{v}} 1.2$ density decreases slowly with maturation, whereas $\mathrm{Na}_{\mathrm{v}} 1.6$ is not detected from early on. It is suggested that both subtypes bind to both ankG isoforms, but the lack of $\beta I V$-spectrin does not allow for stabilization of $\mathrm{Na}_{\mathrm{v}} 1.2$ and recruitment of $\mathrm{Na}_{\mathrm{v}} 1.6$ with maturation. This supports the role of $\beta I V$-spectrin as a stabilizer and recruiter of VGSCs. Additional experiments with isoform specific ankG knockout mice labeled either for $\mathrm{Na}_{\mathrm{v}} 1.6$ or $\mathrm{Na}_{\mathrm{v}} 1.2$, would allow a direct proof, if both channel subtypes bind to both ank $\mathrm{G}$ isoforms or a differential mechanism needs to be taken into account.

So far, an extended model is presented in figure 55. For example, explicit results support matural and structural differentiation of $\beta I V$-spectrin isoforms. Other suggestions, such as structural differentiation of ankG isoforms still need to be examined more closely. Additionally to the presented results, which allowed the extension of a basic model, the thesis demonstrates how much more questions within the field of AIS functionality, maturation and structural organization are unanswered and have not been considered within any study. 


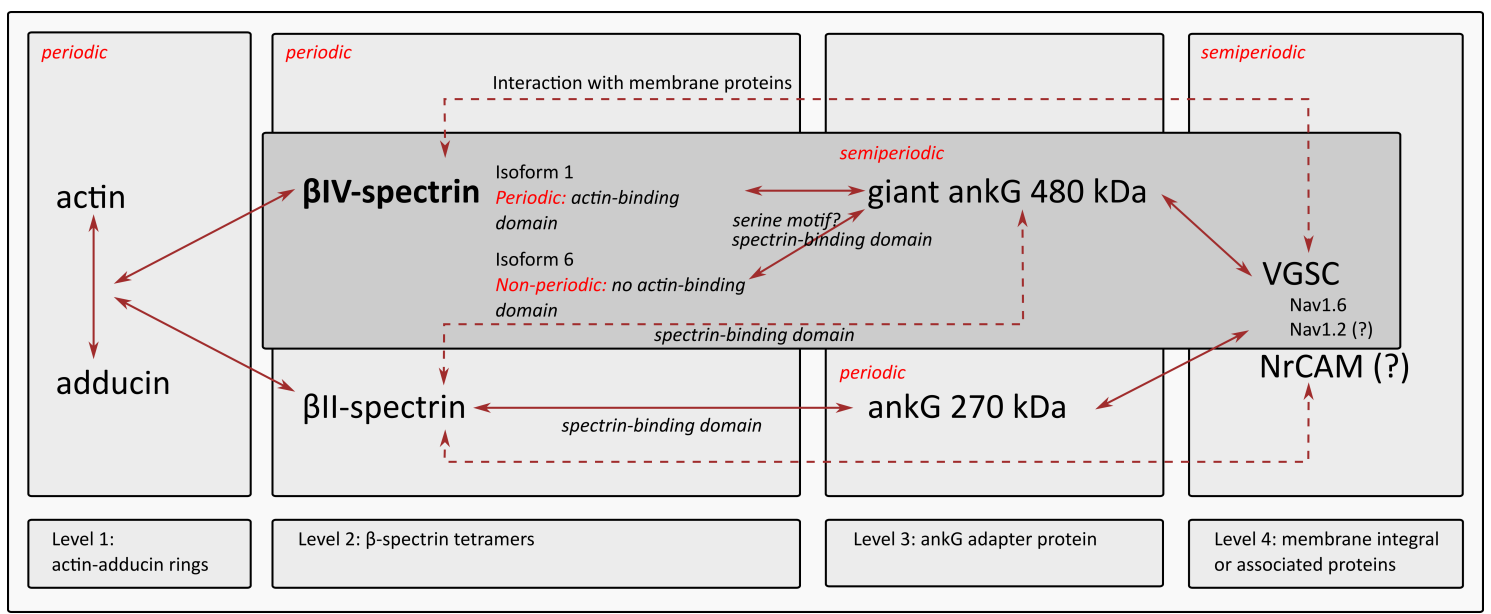

Figure 55: Extended model of AIS proteins and their relationship (dark red arrows) to each other. Four levels of organization (light grey boxes) and their structural organization (red: "periodic", "semiperiodic" and "nonperiodic") are highlighted. Level 1: Cytoskeletal network composed of actin-adducin rings, forming the underlying periodic network. [11 Level 2: $\beta$-spectrin tetramers [16] [53 connecting actin-adducin rings with each other. [11] $\beta I V$-spectrin is highlighted with its main neuronal isoforms, $\beta \operatorname{IV} \sum 1$ and 6 . 16 18 -spectrins have been found organized in a periodic matter, except for $\beta I V$-spectrin C-terminal labeling. Persuming periodically organized main $\beta \operatorname{IV} \sum 1,18 \mathbb{1 1}$ due to an actin-binding domain, and non-periodically organized $\beta \operatorname{IV} \sum 6$, due to a lack of an actin-binding domain. 126 This isoform transfers its non-periodic placement to Level 3 and 4. Level 3: Adapter protein ankG found in a periodic and non-periodic matter. This is persumable due to the different $\beta I V$-spectrin isoforms and binding positions of ankG on the $\beta I V$-spectrin tetramer. Further, differential binding of the two main isoforms giant $480 \mathrm{kDa}$ and $270 \mathrm{kDa}$ ankG to periodic $\beta I I-s p e c t r i n, \beta I V \sum 1$ and non-periodic $\beta I V \sum 6$. Level 4: Membrane integral and associated proteins with an ankyrin-binding domain. The organization of sodium channels and specifically the subtype $\mathrm{Na}_{\mathrm{v}} 1.6$ has been found as (semi-)periodic, 11 transferred from an incomplete periodic spectrin network. $\mathrm{Na}_{\mathrm{v}} 1.2$ and $\mathrm{NrCAM}$ have not explicit been revealed as periodically or (semi-)periodically organized (marked by question marks). Additionally, direct interactions of VGSCs and NrCAM with spectrins are marked (VGSCs: ${ }^{[37}, \mathrm{NrCAM}[130]$ ). Mutation: $\beta$ IV-spectrin, the place of frameshift mutation is highlighted (bold style). [1] Dark grey box highlights the affected protein sequence located in Level 2 - 4, whereas a different influence on different membrane integral and associated proteins is observed. Disruption of the network, due to a $\beta I V$-spectrin lack, affects ankG (contrariwise: $10,[64$ ) and is transferred to some ankG binding proteins, such as VGSCs, but not NrCAM. 


\section{Appendix A: Experimental procedure}

\subsection{The specificity of immunohistochemistry}

One common method to test for antibody specificity is to use multiple antibodies against the same protein, but targeting different epitopes. ${ }^{124}[125$ In this work four different antibodies against $\beta I V$-spectrin, three against ankG, three against the channel subtype $\mathrm{Na}_{\mathrm{v}} 1.6$ and two against the channel subtype $\mathrm{Na}_{\mathrm{v}} 1.2$ were tested.

\subsection{1 $\beta I V$-spectrin antibodies}

Four polyclonal antibodies were used to target different epitopes of $\beta I V$-spectrin (list: 11.2.

1. OriGene, N-terminal sequence aa2-14

2. ATLAS, central located sequence aa1142-1235

3. Rasband lab, Baylor College of Medicine, C-terminal sequence aa2237-2253

4. Santa Cruz, C-terminal sequence aa2350-2400

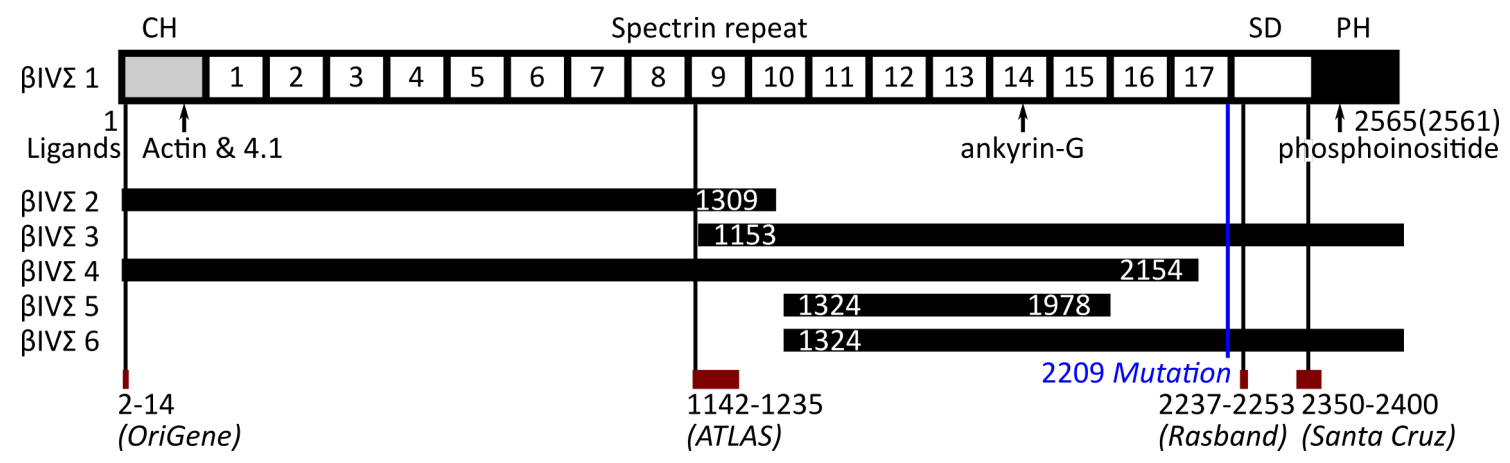

Figure 56: A schematic view of the $\beta I V$-spectrin isoforms. $\beta \operatorname{IV} \sum 1$ is shown in detail. $\beta$ IV $\sum 2-\beta I V \sum 6$ aligned to $\beta I V \sum 1$. Amino acid sequences of isoforms do not correspond completely. Short insertions, deletions and extensions of $\beta \operatorname{IV} \sum 2-\beta \operatorname{IV} \sum 6$ relative to $\beta \operatorname{IV} \sum 1$ are ignored. White numbers indicate the relative starting and ending amino acid of each isoform in relation to $\beta \operatorname{IV} \sum 1$. Ligand binding sites are marked. $\mathrm{CH}$, calponin homology domain; SD, specific domain; PH, pleckstrin homology domain, modified and extended [18] Uniprot.org: Q9H254, Q8VBX2. Sequence parts, where antibodies are raised against, are displayed (red) (list: 11.2 Position of $q v^{3 J}$ frameshift mutation is marked (blue).

$\beta I V$-spectrin antibodies have overlapping AIS labeling. Minor non-specific properties are found.

All double labelings show a significant overlaying staining at the AIS (figure: 57). The central antibody results in a fluorescent dot in the nuclear region of the neuron (figure: 58 . Also, in $q v^{3 J}$ 
mutant neurons, which lack $\beta I V-s p e c t r i n$, the fluorescent dot remains. This suggests that it is non-specific. The structure looks similar to structures known from cilia, which indicates that the $\beta I V-s p e c t r i n$ antibody targets a homologe sequence. This was not investigated further.

All $\beta I V$-spectrin antibodies reveal a weak somatodendritic staining. First of all, it is important to determine the fluorescence intensity of the dendrites staining. This was performed for three out of the four antibodies (N-terminal, central, C-terminal labeled (antibody gift from Rasband lab, Baylor College of Medicine)). Control neurons labeled against $\beta I V-s p e c t r i n$ reveal mean fluorescence intensities in the dendrites: N-terminal labeled 47 a.u., central labeled 28 a.u. and C-terminal labeled (antibody gift from Rasband lab, Baylor College of Medicine) 17 a.u. (calculated from $\mathrm{N}=5$, dark background subtracted). Previously, $\beta \mathrm{IV}$-spectrin has been described as being exclusively found in the AIS. This conclusion is based on immunohistochemistry experiments in slices among others derived from the hippocampal brain region. A strong staining of the AIS, but not dendritic, was observed. ${ }^{[16}$ It is unknown, if a detailed evaluation of the dendritic labeling has been performed, or if the conclusion is only based on observations. The label specificity, more precise the specificity of the applied antibody against the $\beta I V$-spectrin antigen, was proven by using a competitive antigenic peptide. However, no experimental proof has been performed, if $\beta I V$-spectrin is restricted to the AIS. Consequently, it cannot be finally excluded that $\beta I V$-spectrin is found at a low concentration in the dendrites. Helpful are the experiments performed with the $q v^{3 J}$ mutant neurons. Here $\beta I V-$ spectrin is found to be gone in the AIS of DIV 14 neurons. The dendritic labeling is found: N-terminal labeled 6 a.u., central labeled 128 a.u. und C-terminal labeled (antibody gift from Rasband lab, Baylor College of Medicine) 70 a.u. (calculated of $\mathrm{N}=$ 5 , dark background subtracted). The comparison of the dendritic labeling in control and mutant neurons reveals small differences. For N-terminal labeling a slight decrease, but for C-terminal and central labeling a slight increase is observed. If one assumes that $\beta I V$-spectrin would be part of the dendrites, then a clear reduction or even an absence of $\beta I V$-spectrin labeling in mutant dendrites would be expected, due to $\beta I V$-spectrin loss in $q v^{3 J}$ mutant neurons. This is not the case and allows to suggest a $\beta I V$-spectrin absence in dendrites. Moreover, the results suggest that the antibodies have a low level of non-specific binding. Furthermore, autofluorescence of the neurons needs to be considered. To conclude, the comparison of the mean fluorescence intensities in the dendrites and AIS reveals that the intensities in dendrites of 6 to 47(128) a.u. are minor. 

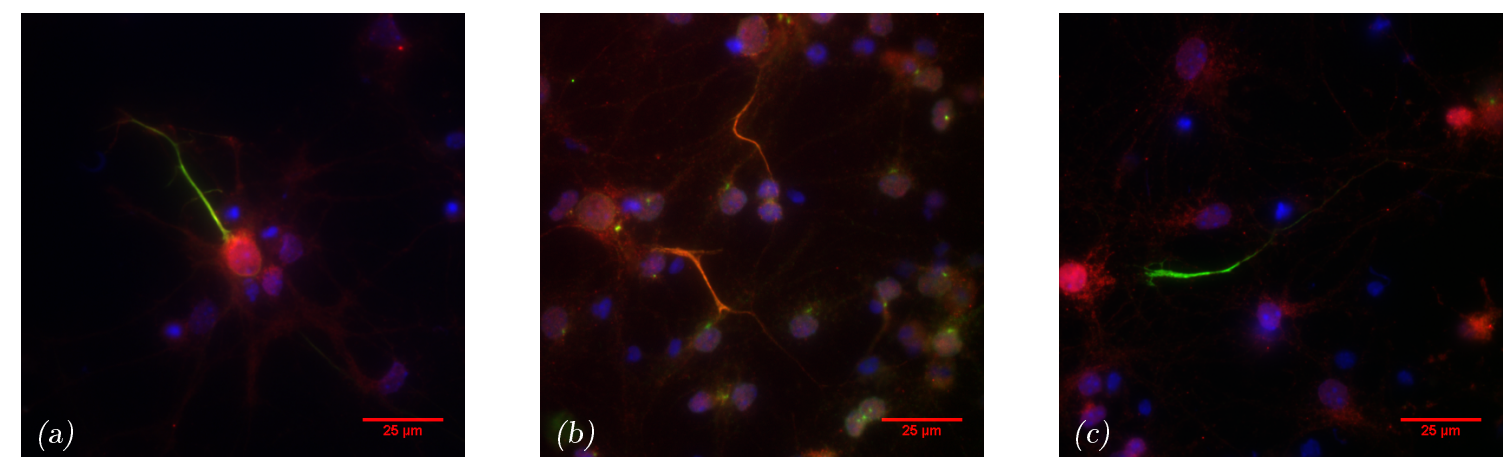

Figure 57: Example images of $\beta I V-s p e c t r i n$ double labeling. (a) N-terminal (red) and C-terminal (antibody gift from Rasband lab, Baylor College for Medicine) (green) at DIV 13, (b) N-terminal (red) and central (green) at DIV 20, (c) C-terminal (C-terminal antibody, sc-131789, 11.2 (red) and C-terminal (antibody gift from Rasband lab, Baylor College for Medicine) (green) at DIV 13. Nucleus stained by DAPI (blue). Scale bar: $25.0 \mu \mathrm{m}$.

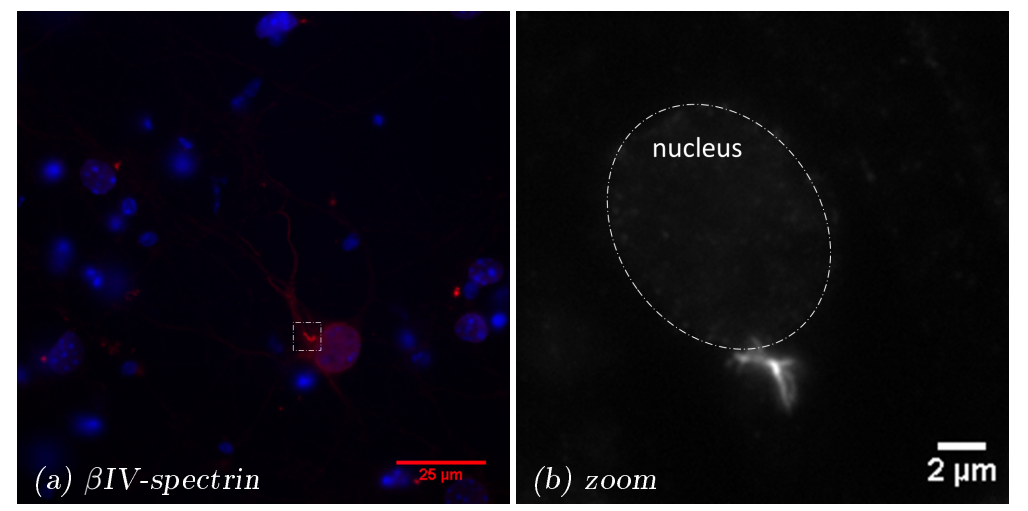

Figure 58: Example image of a hippocampal neuron at DIV 20. (a) Wide-field image of $\beta I V$-spectrin labeled central (red) and nucleus stained by DAPI (blue). Area of uncertain fluorescence is boxed. (b) Zoom in a neuron. Nucleus is marked by dashed lines. Area of uncertain fluorescence close to the nucleus (somatic located). Scale bar: $25.0 \mu \mathrm{m}$. 


\section{$\beta I V$-spectrin antibodies target different splice variants.}

The four antibodies target different splice variants of $\beta I V$-spectrin. This was examined more closely by performing double labeling and extracting the profiles (method: 3.5.1p.

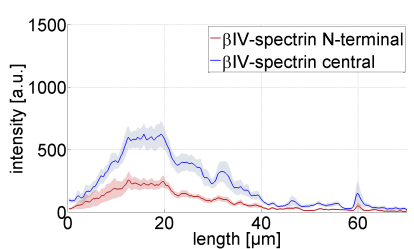

(a) $D I V 8$

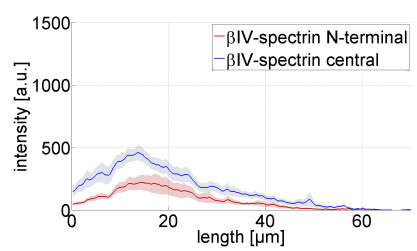

(b) $D I V 13$

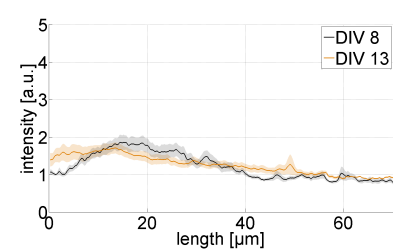

(c) Profiles divided

Figure 59: Double labeling of $\beta I V$-spectrin at an N-terminal and a more central located sequence part. (a) DIV $8, \mathrm{~N}=12$. (b) $D I V 13, \mathrm{~N}=9$. (c) Profiles divided: $\beta I V$-spectrin central by N-terminal. Error is the SEM.

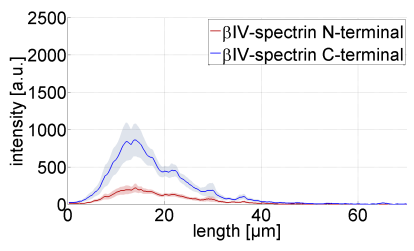

(a) $D I V 8$

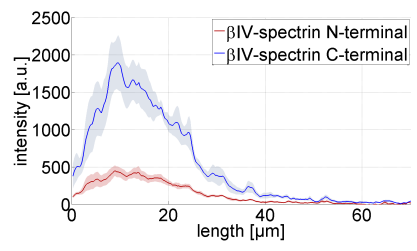

(b) $D I V 13$

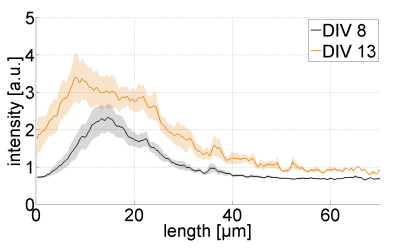

(c) Profiles divided

Figure 60: Double labeling of $\beta I V-s p e c t r i n$ at a N-terminal and C-terminal (antibody gift from Rasband lab, Baylor College for Medicine) located sequence part. (a) $D I V 8, \mathrm{~N}=15$. (b) $D I V 13, \mathrm{~N}=11$. (c) Profiles divided:

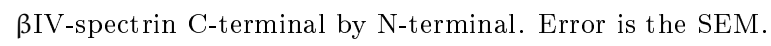

In the two weeks the profiles (figure: 59 60 look as expected. Profiles along the AIS with areas of high mean fluorescence intensities are found, which suggests an enrichment of $\beta I V$-spectrin. With maturation the $\beta I V$-spectrin density increases. Double labeling allows to determine if the antibodies target the same structures. Profiles were divided neuron by neuron. Afterward, the mean profile and the standard error of the mean (SEM) was calculated (method: 3.5.1). After division of profiles, central labeled by N-terminal labeled, the mean profile appears smooth (figure: 591. Concluding, the antibodies target the same splice variant ( $\left.\beta I V \sum 1\right)$. After division of the profiles from double labeling $\mathrm{N}$ - and C-terminal an uneven profile remains. To conclude, the antibodies target different structures. Both target the $\beta \operatorname{IV} \sum 1$, but only the antibody directed against a C-terminal located sequence targets the $\beta \operatorname{IV} \sum 6$. 


\subsubsection{Ankyrin-G antibodies}

The adapter protein ankG is known to be highly enriched in the AIS. Three different antibodies against ankG were evaluated (all from Santa Cruz Biotechnology, list: 11.2.).

1. Monoclonal, spectrin-binding domain, synthetic peptide aa982-1107

2. Polyclonal, C-terminal, aa4150-4200

3. Polyclonal, C-terminal, aa4163-4377

\section{AnkG antibodies show overlapping AIS labeling.}

The N- and C-terminal antibodies result in a bright AIS and additional somatic staining (figure: 61. AnkG has been found to be a major part of the AIS, but not excluded from the soma (e.g. $82 \sqrt[83]{83}$ ). The C-terminal antibodies display higher somatic fluorescence intensities than the N-terminal antibody. This is not surprising, since the N-terminal antibody is a high specific monoclonal antibody, whereas the C-terminal antibodies are polyclonal.
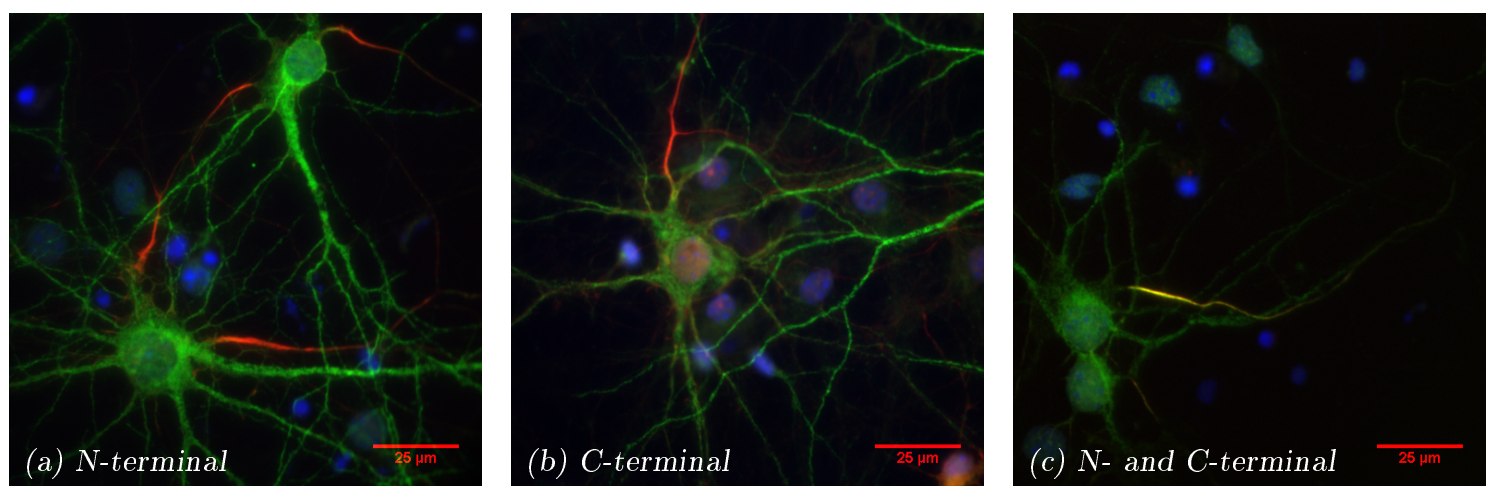

Figure 61: Comparison of two ankG (red) antibodies targeting either a more N- or C-terminal (C-terminal antibody, sc-28561, 11.2 located region. Nucleus stained by DAPI (blue). (a) and (b) double labeling with MAP2 (green) to identify the AIS. (c) Overlapping fluorescence at the AIS: C-terminal (green) and N-terminal (red).

\section{AnkG antibodies target the same splice variants.}

Database research (www.uniprot.org, Q12955, W6PPA1) (data not shown) reveals that the neuronal ankG isoforms (270 kDa, $480 \mathrm{kDa})$ differ significantly in sequence length, but share a common N- and C-terminal region. The alignment (tool: EMBL-EBI EMBOSS) shows that the three antibody epitopes are found in both ankG isoforms.

Double labeling was performed for control and $q v^{3 J}$ mutant neurons (figure: 62. The $q v^{3 J}$ mutant neurons have a significant lower ankG density (results: 6.1). The profiles divided cell-by-cell appear smooth. Consequently, confirmed by control and $q v^{3 J}$ mutant neurons, the antibodies target all splice variants. 


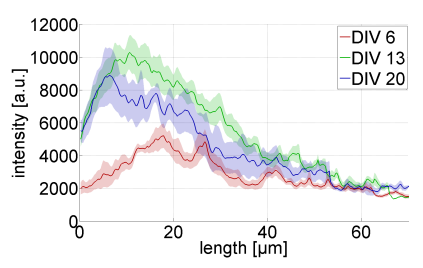

(a) control N-terminal

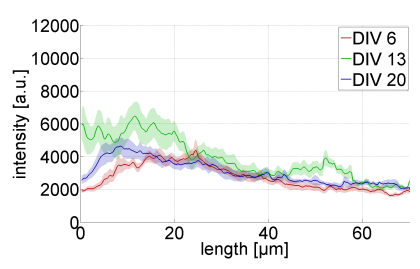

(d) mutant N-terminal

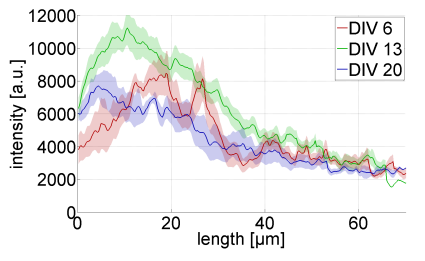

(b) control C-terminal

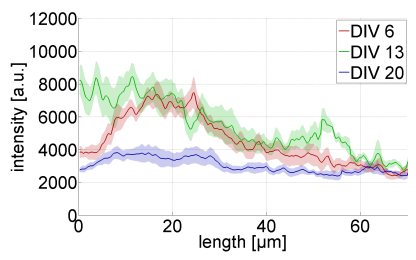

(e) mutant C-terminal

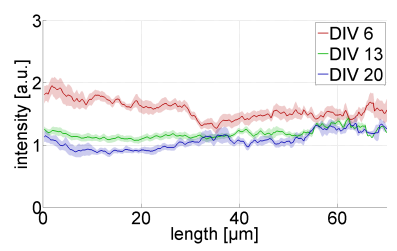

(c) control divided

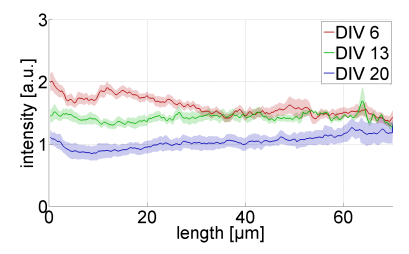

(f) mutant divided

Figure 62: Double labeling of ankG at a N-terminal and C-terminal (C-terminal antibody, sc-28561, 11.2 located sequence part over three weeks of maturation at DIV 6, DIV 13, and DIV 20. (a) Control labeled N-terminal: AnkG is enriched in the AIS. (b) Control labeled C-terminal: AnkG is enriched in the AIS. (c) Control divided profiles, C-terminal by N-terminal: Profiles appear smooth. Antibodies target the same structures. (d) Mutant labeled N-terminal: A weak, but constant ankG density is found. (e) Mutant labeled C-terminal: A weak, but constant ankG density is found. (f) Mutant divided profiles, C-terminal by N-terminal: Profiles appear smooth. Antibodies target the same structures. $\mathrm{N}($ control $)=8,13,6 . \mathrm{N}$ (mutant) $=12,9,5$. Error is the SEM. 


\subsubsection{Subtype $\mathrm{Na}_{\mathrm{v}} 1.6$ antibodies}

VGSC subtype labeling is known to be a challenging field. Here three different antibodies against the sodium channel subtype $\mathrm{Na}_{\mathrm{v}} 1.6$ were evaluated (list: 11.2).

1. Polyclonal, from Alomone, epitope corresponding to the aa1042-1061 of $\mathrm{Na}_{\mathrm{v}} 1.6$ in rats. This sequence is located in the intracellular loop between the domains II and III.

2. Monoclonal, from Neuromab, raised against a synthetic peptide corresponding to the aa459476 of $\mathrm{Na}_{\mathrm{v}} 1.6$ in rats. This sequence is located in the intracellular loop between the domains II and III.

3. Gift from the Rasband lab, Baylor College of Medicine.

The antibodies were tested under different conditions, including a wide range of concentrations, two different immunohistochemistry protocols and antigen retrieval methods, SDS and pepsin treatment. Changes in immunohistochemistry protocols and antigen retrieval did not improve the staining quality or led to different results (data not shown).
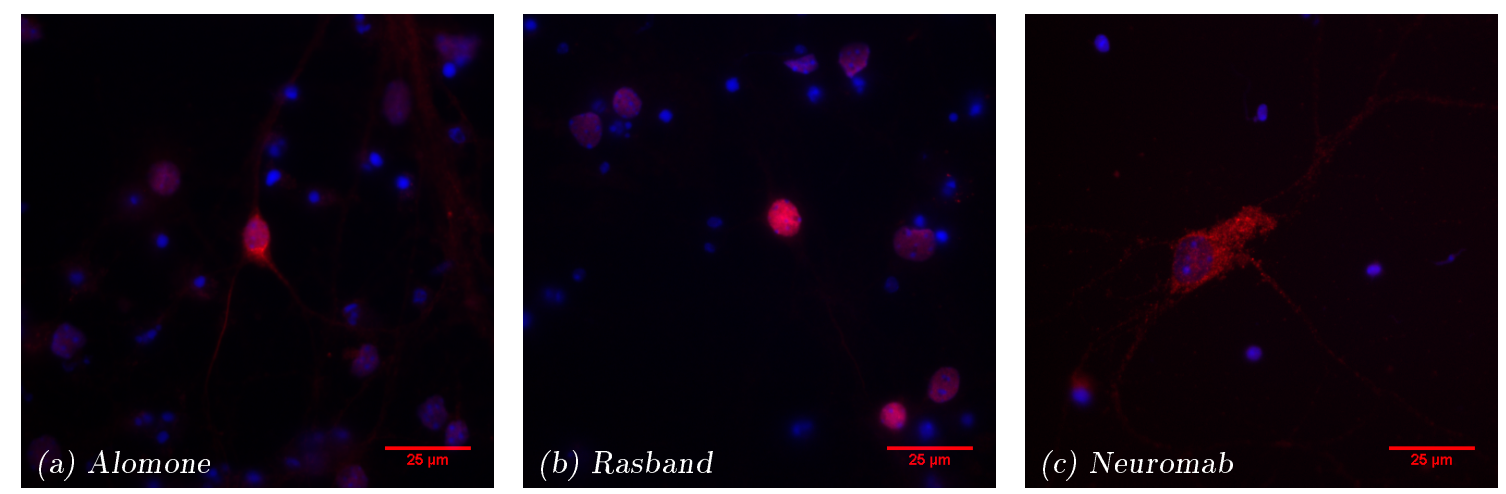

Figure 63: Comparison of three different anti-Na 1.6 (red) antibodies at DIV 20. Nucleus stained by DAPI (blue). (a) Polyclonal Nav1.6 antibody from Alomone (b) Nav1.6 antibody from Rasband lab (c) Monoclonal $\mathrm{Na}_{\mathrm{v}} 1.6$ antibody from Neuromab.

\section{Diversity of staining pattern suggests non-specific binding of the antibodies.}

Major differences between the antibodies were observed.

In most cases the Alomone antibody shows a significant AIS staining (identified by double labeling with anti-MAP2, anti-panNa $\mathrm{v}_{\mathrm{v}}$ or anti-ankG). A weaker staining is observed for somatodendritic compartments. $\mathrm{Na}_{\mathrm{v}} 1.6$ channels have been described in the AIS and somatic compartment before. 26 27 However, a bright nucleus staining, as obtained by this antibody, seems inappropriate. Moreover, this staining has been found to remain in $\mathrm{Na}_{\mathrm{v}} 1.6$ knockout mice (conversations with Anja Scheller, University of Saarland). This suggests that it is non-specific. Additionally, when 
analyzing the fluorescence intensity in the somatic region an overestimation needs to be considered, since the bright nucleus staining might influence the somatic mean fluorescence intensity. The antibody, which was obtained as a gift from the Rasband lab, Baylor College on Medicine displays a bright nucleus staining and a weak staining of neuronal branches. No images with a significant AIS staining were obtained. The bright nucleus and very weak neurite staining indicate that it is non-specific. Especially, the bright nucleus suggests a similar non-specific binding, as described for the Alomone antibody.

The monoclonal antibody from Neuromab shows a very diverse staining. In some cases the staining failed completely and in other cases an evenly staining of the neurons was observed. ${ }^{131}$ In these cases a significant somatic staining was obtained. This seems appropriate, since $\mathrm{Na}_{\mathrm{v}} 1.6$ channels have been described in somatic compartments before. 26] Dendrites and AIS could not be distinguished. This cannot be supported with previous findings, because $\mathrm{Na}_{\mathrm{v}} 1.6$ channels have been described as being enriched in the AIS. $29 \sqrt{31}$ However, the obtained staining pattern agrees with an earlier publication. 131

The epitopes of the Alomone and Neuromab antibodies are located in the same intracellular loop between the domains II - III. Similar staining pattern of these antibodies are expected.

Due to the fact that the Alomone antibody has been best described and its non-specific binding properties have been evaluated in detail, it appears most suitable to be used for the experiments in this thesis. 


\subsubsection{Subtype $\mathrm{Na}_{\mathrm{v}} 1.2$ antibodies}

The VGSC subtype $\mathrm{Na}_{\mathrm{v}} 1.2$ was targeted with two antibodies.

1. Polyclonal, from Alomone, raised against the region aa467-485 of $\mathrm{Na}_{\mathrm{v}} 1.2$ in rats.

2. Monoclonal, from Neuromab, raised against aa1882-2005 of $\mathrm{Na}_{\mathrm{v}} 1.2$ in rats. The sequence is located at the cytoplasmic C-terminus.

The polyclonal antibody from Alomone shows a bright staining at or close to the nucleus. A weak staining is observed for the neuronal branches. Dendrites and axon cannot be distinguished. The monoclonal Neuromab antibody shows a brighter and in most cases significant AIS staining. This seems appropriate, since $\mathrm{Na}_{\mathrm{v}} 1.2$ channels have been described as being enriched in the AIS. 29 One explanation for the difference is that the polyclonal has a lower specificity than the monoclonal antibody. Moreover, masking of one or the other epitope in the AIS cannot be excluded.

Identification of the AIS was performed by double labeling with anti-MAP2 or anti-ankG.

The monoclonal anti-Na 1.2 antibody from Neuromab was chosen for the project.
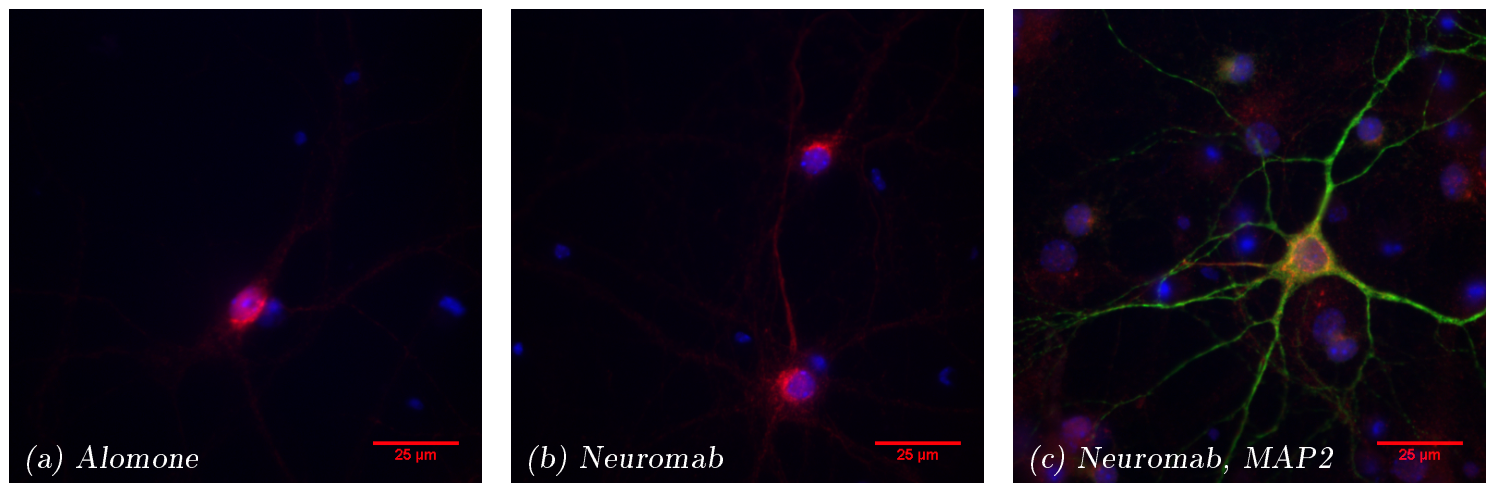

Figure 64: Comparison of two different anti-Nav 1.2 (red) antibodies at DIV 13. (a) Polyclonal $\mathrm{Na}_{\mathrm{v}} 1.2$ (red) antibody from Alomone (b) Monoclonal Na 1.2 (red) antibody from Neuromab. (c) Double labeling of $\mathrm{Na}_{\mathrm{v}} 1.2$ (red) antibody from Neuromab with anti-MAP2 (green) for AIS identification. 


\section{Appendix B: Results}

The following appendix section displays additional results, which are not shown in the main part of the thesis, but support the discussion section and drawn conclusions.

\section{$10.1 \beta I I-s p e c t r i n$}

\section{$\beta I I-s p e c t r i n$ does not rescue for $\beta I V$-spectrin deficiency.}

$\beta$ II-spectrin belongs to the spectrin family. ${ }^{[27}[132]$ Rescue mechanisms between spectrins have been described before. ${ }^{78}$ Thus, the question arises if the loss of $\beta I V$-spectrin in the $q v^{3 J}$ mutation has an effect on $\beta I I-$-spectrin density.

The AIS was identified by double labeling with ankG (C-terminal antibody, sc-28561, 11.2). The images (figure: 65 do not show a reduced staining of $\beta I I-$ spectrin in $q v^{3 J}$ mutant neurons. The average intensity of the AISs was determined. Control and $q v^{3 J}$ mutant neurons have a high $\beta I I-s p e c t r i n$ density in the first week. While $\beta \mathrm{IV}$-spectrin increases only in control neurons, $\beta \mathrm{II}-$ spectrin decreases 111 and behaves similar in control and $q v^{3 J}$ mutant neurons. Consequently, $\beta I I-s p e c t r i n$ does not function within a rescue role for $\beta I V$-spectrin.
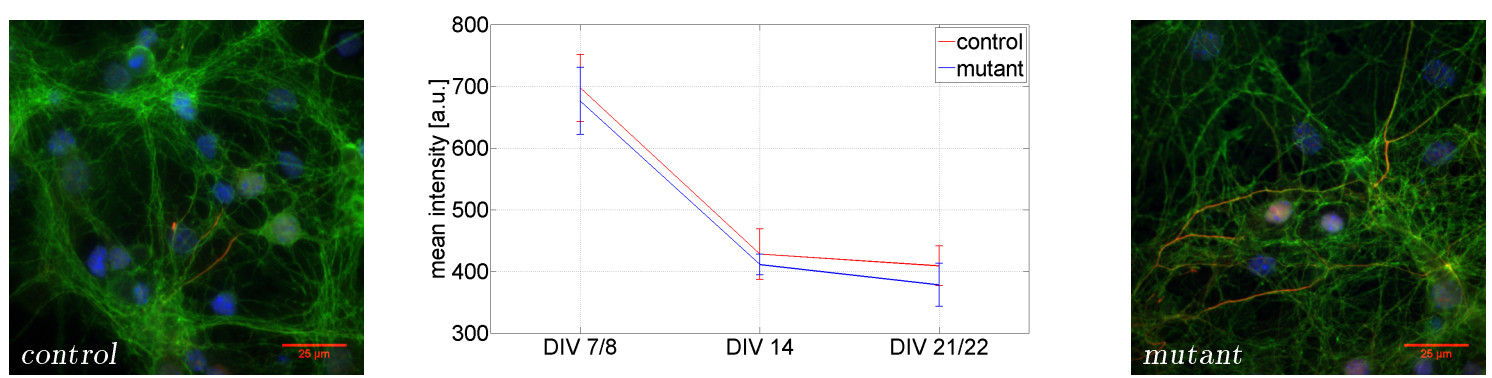

Figure 65: $\beta I I-s p e c t r i n$ in control and $q v^{3 J}$ mutant neurons. Double labeling of $\beta I I-s p e c t r i n$ (green) and ankG (red) (C-terminal antibody, sc-28561, 11.2 at DIV 8. Nucleus stained by DAPI (blue). Scale bar: $25.0 \mu \mathrm{m}$. Graph: $\beta I I-s p e c t r i n$ expression in control and $q v^{3 J}$ mutant neurons at three maturation stages. The graphic shows the mean fluorescence intensities [a.u.] at the AIS within the distance $0-50.0 \mu \mathrm{m}$ from the soma or first branching point. $\mathrm{N}($ control $)=36,22,27 . \mathrm{N}($ mutant $)=32,11,22$. The error is the SEM. 
Periodic organization of $\beta$ III-spectrin is not restricted to the AIS.

$\beta I I-s p e c t r i n$ is not exclusively expressed in the AIS, but a major component of somatodendritic compartments. $127 \sqrt[132]{132}$ The AISs of mature neurons have a clear periodic organization of $\beta$ IIspectrin (figure: 66. The spatial frequency is about $190 \mathrm{~nm}$ and agrees with previous findings. 111 Here, a detailed analysis revealed periodic organized branches, negative for $\beta I V$-spectrin and ankG, which indicates that these are not AISs. Extracted power spectra confirm the periodic organization. Previously periodicity has been described as being confined to the AIS [11, later this has been rejected and periodicity has been described in dendrites. $\frac{}{12}$ Here a clear periodic $\beta I I-s p e c t r i n$ organization in dendrites is found.
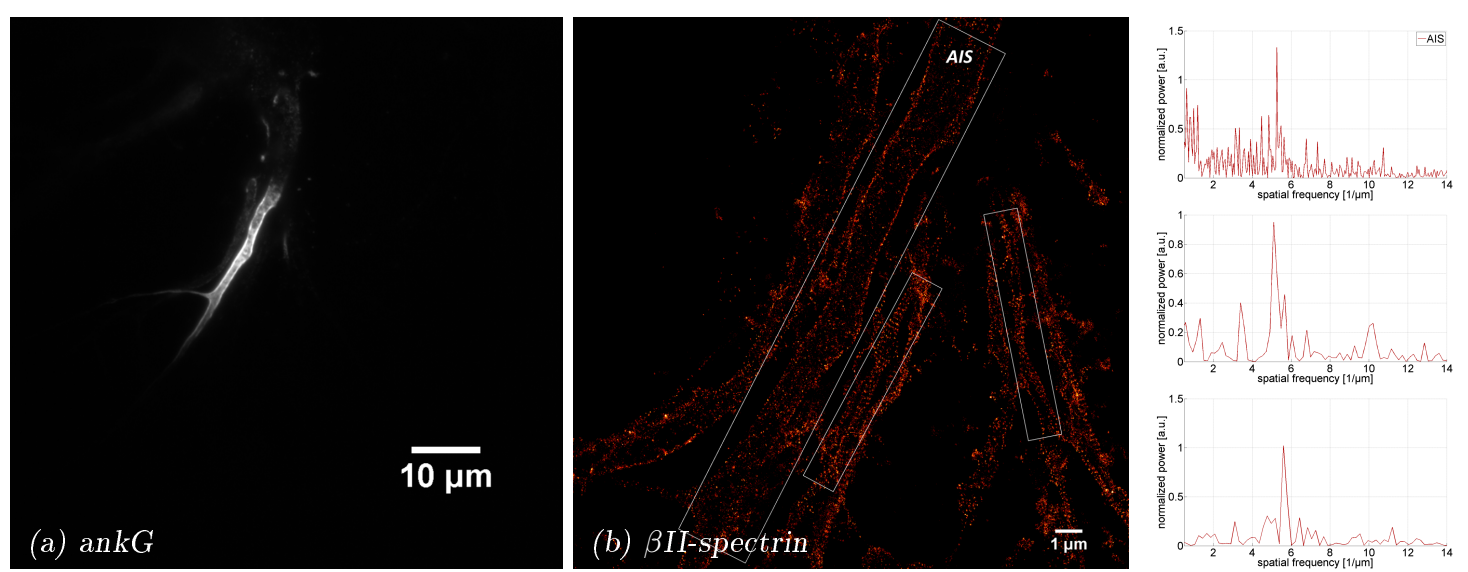

Figure 66: Structure of $\beta I I-s p e c t r i n$ in neurites of control neurons. (a) AnkG (C-terminal antibody, sc-28561, 11.2 identifies the AIS. (b) Complete STORM image of a hippocampal neuron at DIV 13 stained for $\beta$ II-spectrin. The AIS and two potential dendrites are marked. The corresponding power spectra reveal frequencies in the range of 165 - 192 nm. (AIS: top, Dendrites: bottom) 


\section{$Q v^{3 J}$ mutation does not disrupt the $\beta I I-$ spectrin structure.}

Imaging was performed on DIV 13 neurons. Control and mutant neurons have periodically structured AISs (figure: 67) and similar power spectra. In both spectra, broad peaks within the range of 180 to $205 \mathrm{~nm}$ are observed (figure: 67). There is no influence of the $q v^{3 J}$ mutation on the $\beta I I-$-spectrin structure.
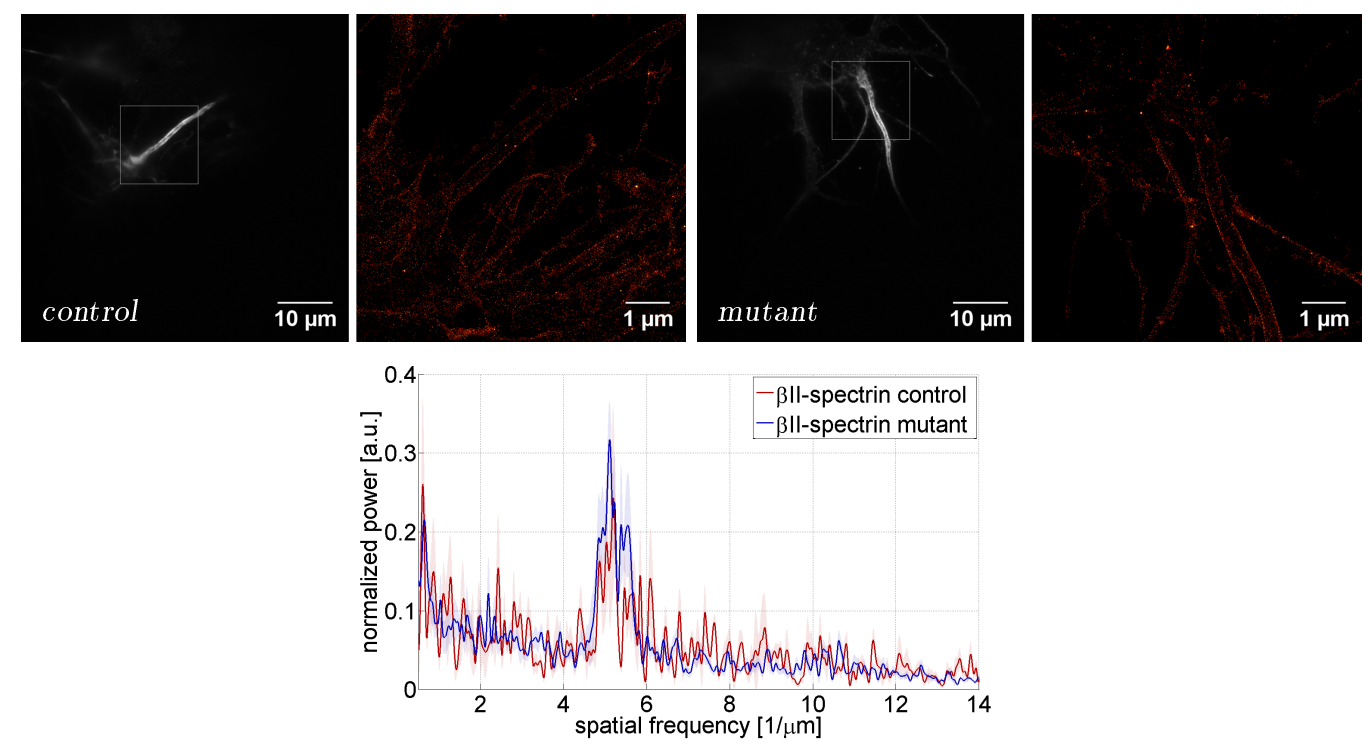

(a) Calculated power spectra from the complete $\beta$ IIspectrin data sets.

Figure 67: Structural comparison of $\beta I I-s p e c t r i n$ in control and $q v^{3 J}$ mutant neurons at DIV 13. AnkG channel (C-terminal antibody, sc-28561, 11.2 shown in grey. Boxed region of interest was imaged for $\beta$ II-spectrin in control and $q v^{3 J}$ mutant neurons. (a) Power spectra calculated from the complete data sets. $\mathrm{N}=25,14$. Error is the SEM. 


\subsection{The structure of ankG targeted C-terminal in immature neurons}

Previously, $\beta I V$-spectrin was described as appearing periodic in immature, but not in mature neurons, when labeled at a C-terminal located epitope (results: 34). Analog, ankG is investigated by C-terminal labeling in immature neurons.

\section{Low level of periodically organized ankG in immature control and $q v^{3 J}$ mutant neurons.}

The extracted power spectra are noisy (figure: 69p. High power values are prominent at high frequencies. Between the frequencies 172 and $238 \mathrm{~nm}$ display control and $q v^{3 J}$ mutant neurons a peak in the power spectrum, which indicates that some AISs or regions have a periodic organization. The pattern is more prominent in $q v^{3 J}$ mutant than in control neurons (as observed for $\beta I V-$ spectrin labeled N-terminal (results: [51, discussion: 7.2p). Contrariwise, in mature wildtypes no periodic organization of ankG, labeled C-terminal, was observed (results: 69).

The obtained data set is classified in three groups:

1. Subgroup 1: Prominent $200 \mathrm{~nm}$ organization (Control neurons $4.5 \%$, mutant neurons $37.0 \%)$.

2. Subgroup 2: Partly $200 \mathrm{~nm}$ organization. Partly the spacing between localizations is above $200 \mathrm{~nm}$ (Control neurons $13.6 \%$, mutant neurons $18.5 \%$ ).

3. Subgroup 3: Noise only (Control neurons $81.8 \%$, mutant neurons $44.4 \%$ ).

Here arisis the question, why ankG labeled at its C-terminus displays a partly periodic organization, especially in $q v^{3 J}$ mutant neurons. AnkG has been described to have two possible binding domains for spectrin. The common spectrin-binding domain, located about one fourth from the N-terminus and the more central located serine ${ }^{[73}$ (details: 2.3. . In both cases ankG has multiple options for its orientation. At $D I V 8$ to 10 the density of irregular $\beta \operatorname{IV} \sum 6$ is very low and allows for a more regular organized ankG based on $\beta I V \sum 1$. Further, wide-field data (results: 22 6.1) show that ankG is not necessarily fully expressed within the first week. At the age of DIV 8 to 10 a relative small number of molecules is placed into the network. The sparse molecule placement might allow, despite the tremendous size of giant $480 \mathrm{kDa}$ ankG ${ }^{[73}$, to resolve partly periodic areas. An even more sparse placement of ankG molecules in $q v^{3 J}$ mutant neurons, due to the low density of $\beta \operatorname{IV} \sum 1$, might enhance the periodic pattern. Further, it is assumed that the lower ankG density in $q v^{3 J}$ mutant neurons has a relative higher density of $270 \mathrm{kDa}$ ankG to giant 480 $\mathrm{kDa}$ ankG (discussed in: 8). Since, $270 \mathrm{kDa}$ ankG is much smaller than giant $480 \mathrm{kDa}$ ankG the 
C-terminal labeling position is located closer to the underlying spectrin network.

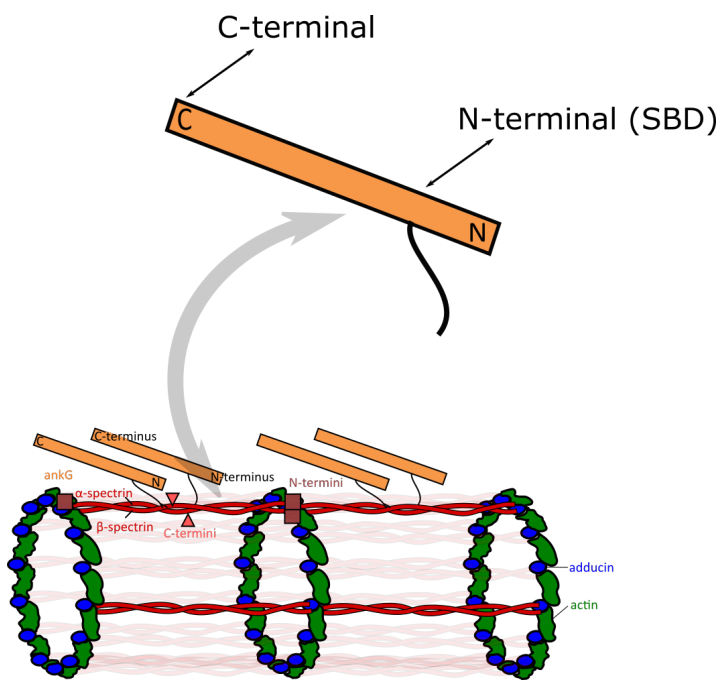

(a) Model of ankG labeling in the AIS of an immature neuron.

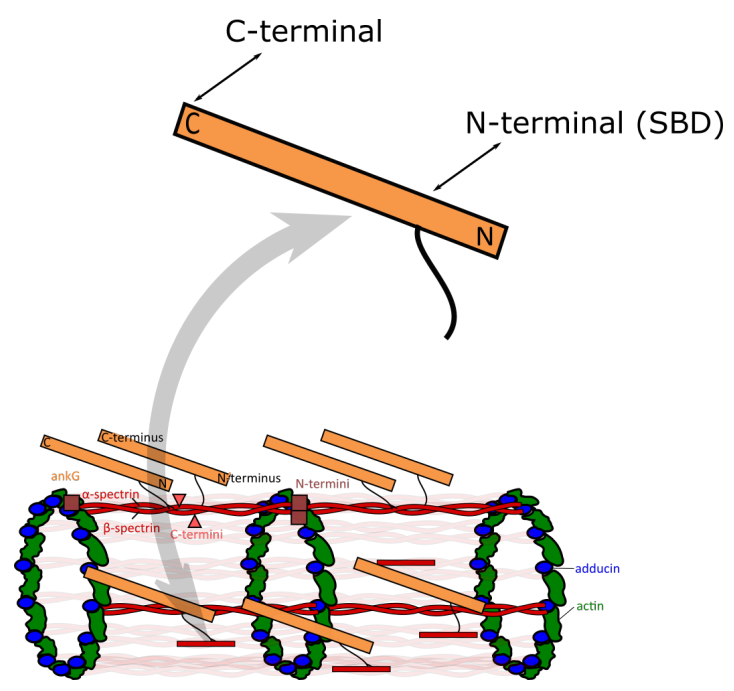

(b) Model ankG labeling in the AIS of an mature neuron.

Figure 68: Model of ankG organization in the AIS. Labeling positions of the N- and C-terminal antibodies are illustrated on the giant $480 \mathrm{kDa}$ ankG molecule. Whereas N-terminal labeling targets the spectrin-binding domain, targets C-terminal labeling the furthest possible position from the spectrin molecules. (a) Immature neurons consist of $\alpha \mathrm{II} / \beta \mathrm{II}$ - and $\alpha \mathrm{II} / \beta \mathrm{IV} \sum 1$-spectrin tetramers, which form a highly organized AIS. In immature $q v^{3 J}$ mutant neurons $\alpha \mathrm{II} / \beta \operatorname{IV} \sum 1$-spectrin tetramers are highly reduced or even absent and the structure is maintained only by $\alpha \mathrm{II} / \beta \mathrm{II}-$ spectrin tetramers. A low pronounced, but significant periodic organization of ankG transferred from the underlying network, can be revealed by far distance C-terminal labeling. (b) Mature neurons consist of $\alpha \mathrm{II} / \beta \mathrm{II}-$ and $\alpha \mathrm{II} / \beta \operatorname{IV} \sum 1$-spectrin tetramers and an additional $\beta \operatorname{IV}$-spectrin splice variant, $\beta \operatorname{IV} \sum 6$. This increases not only the density of spectrin molecules in mature neurons, but also integrates in a non-periodic matter. The organization of ankG appears largely unorganized. 


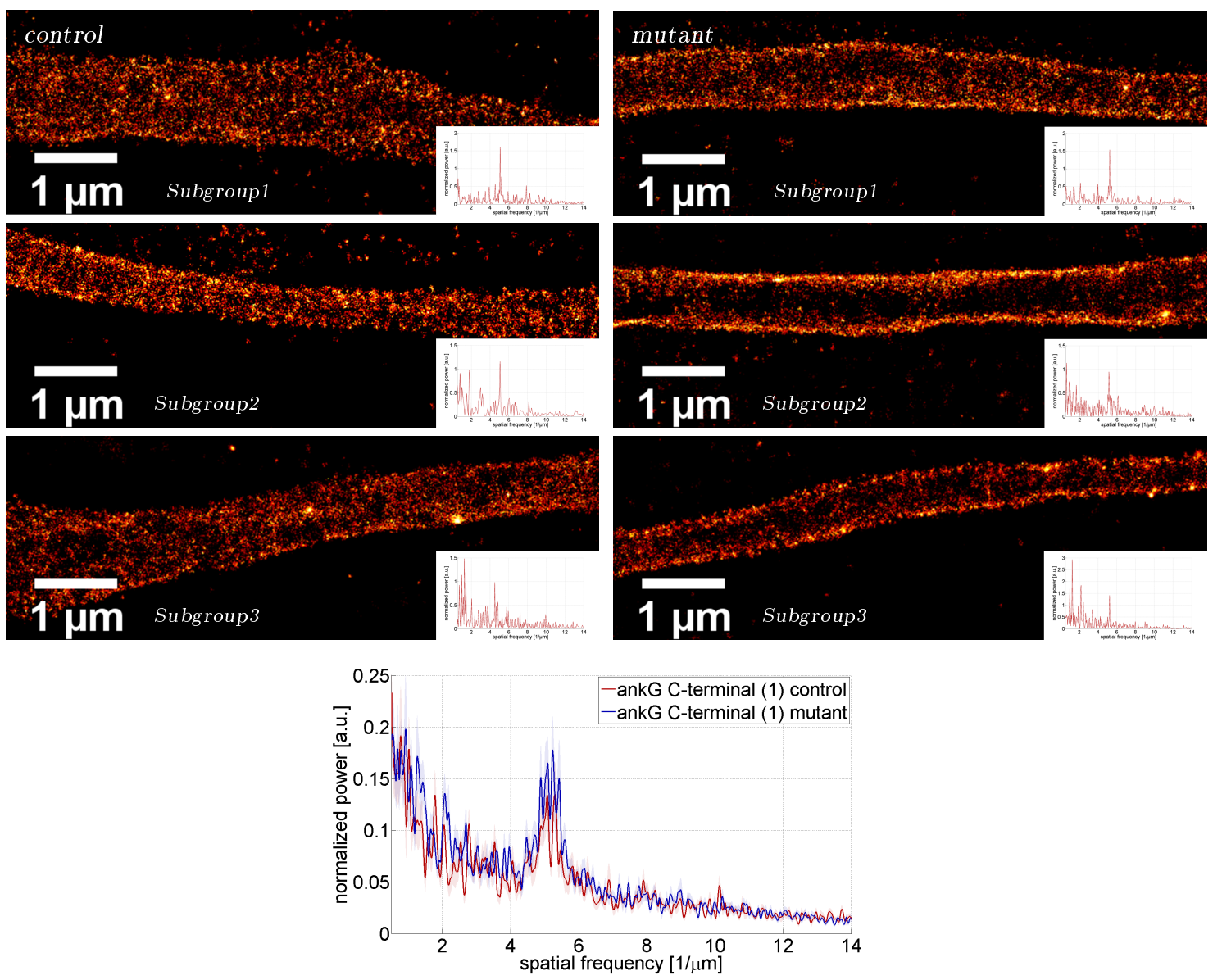

(a) Calculated power spectra from the complete data set.

Figure 69: Structural comparison of ankG (C-terminal antibody, sc-28561, 11.2 in control and $q v^{3 J}$ mutant neurons at DIV $8-10$ by STORM imaging. Example images from three subgroups of the data set and their corresponding power spectra. Subgroup1: Prominent $200 \mathrm{~nm}$ periodic pattern. Subgroup2: Partly areas of $200 \mathrm{~nm}$ periodic organization. Subgroup3: Noise only. (a) Power spectra calculated from the complete data sets for control and $q v^{3 J}$ mutant neurons. Low level of $200 \mathrm{~nm}$ periodic organization, more prominent in $q v^{3 J}$ mutant neurons.

$$
\mathrm{N}=22,27 \text {. Error is the SEM. }
$$




\subsection{Details of voltage-gated sodium channel subtype $\mathrm{Na}_{\mathrm{v}} 1.2$}

The obtained data set ( $D I V$ 14) revealed no clear periodic pattern for $\mathrm{Na}_{\mathrm{v}} 1.2$ channels (results: 5.1. The mean power spectrum shows several peaks at high frequencies. This includes a frequency of about $200 \mathrm{~nm}$, which was identified as the periodic pattern for VGSC organization by anti$\operatorname{panNa}_{\mathrm{v}} 11$ and anti-Nav1.6 (figure: 35 labeling. $\mathrm{Na}_{\mathrm{v}} 1.2$ and $\mathrm{Na}_{\mathrm{v}} 1.6$ share a common ankG binding motif, which leads to the expectation that their organization in the AIS does not differ.

The distribution of the data set is studied in more detail. The power spectra were extracted for each neuron and classified into two groups (figure: 70):

1. Spectra, which have a high power at the spatial frequency of about $200 \mathrm{~nm}$ and at additional higher frequencies.

2. Spectra, which appear noisy and have no peak at about $200 \mathrm{~nm}$.

The groups are equally sized. None of the spectra has a defined frequency of about $200 \mathrm{~nm}$. Thus, averaging of the power spectra is not the reason why no clear pattern of $\mathrm{Na}_{\mathrm{v}} 1.2$ organization is found (discussed in: 5.2 ).

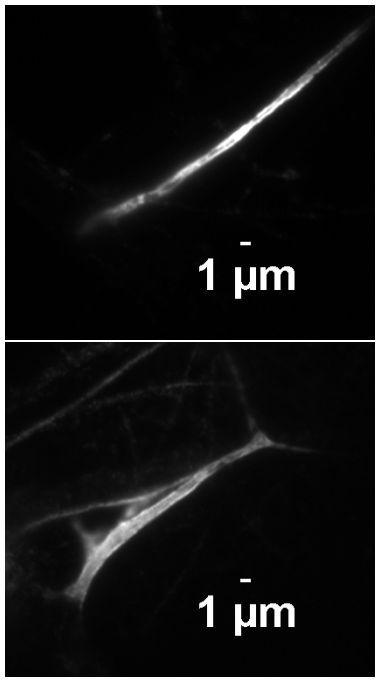

(a) AnkG C-terminal.

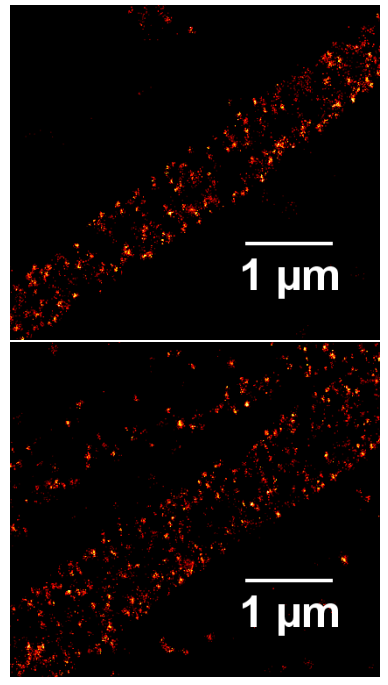

(b) Nav1.2 STORM.
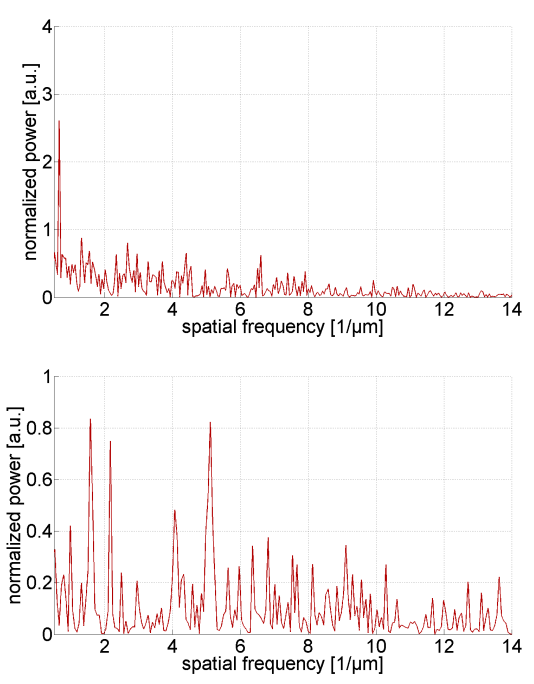

(c) Power spectra.

Figure 70: Details of $\mathrm{Na}_{\mathrm{v}} 1.2$ structural organization by STORM imaging. Example images represent the two identified groups within the data set. (a) AIS identification by ankG (C-terminal antibody, sc-28561, 11.2 widefield image. (b) Zoom in $\mathrm{Na}_{\mathrm{v}} 1.2$ STORM image. (c) Corresponding power spectra for the full length of the AIS. 


\section{$10.4 \quad \mathrm{NrCAM}$}

The neuronal cell adhesion molecule $(\mathrm{NrCAM})$ is a major component expressed in the AIS. It is involved in neuron-neuron adhesion and has, like many other proteins, an ankyrin-binding site. AnkG was described as being affected by the frameshift mutation $q v^{3 J}$ (results: 6.1). Consequently, NrCAM could also be affected.

NrCAM displayed a dense network in both cultures (figure: 71). Double labeling with ankG (labeled at its N-terminus, list 11.2) allowed the AIS identification.

\section{The $q v^{3 J}$ mutation does not affect the NrCAM density.}

The mean intensities were determined for three maturation stages (figure: 71). In immature neurons control neurons have a higher NrCAM density. Within the second week the difference decreases. In the third week of studied maturation, the NrCAM density in $q v^{3 J}$ mutant neurons appears to be higher than in control neurons. The higher $\mathrm{NrCAM}$ density in $q v^{3 J}$ mutant neurons within the third week seems inappropriate, since the effect of the mutation was observed to be minor in immature neurons and major in mature neurons (dicussion: 7.2). Possibly, the culture density falsifies the results (figure: 71). No further experiments were performed in order to investigate, if there is an additional role of $\mathrm{NrCAM}$ within $q v^{3 J}$ mutant neurons.
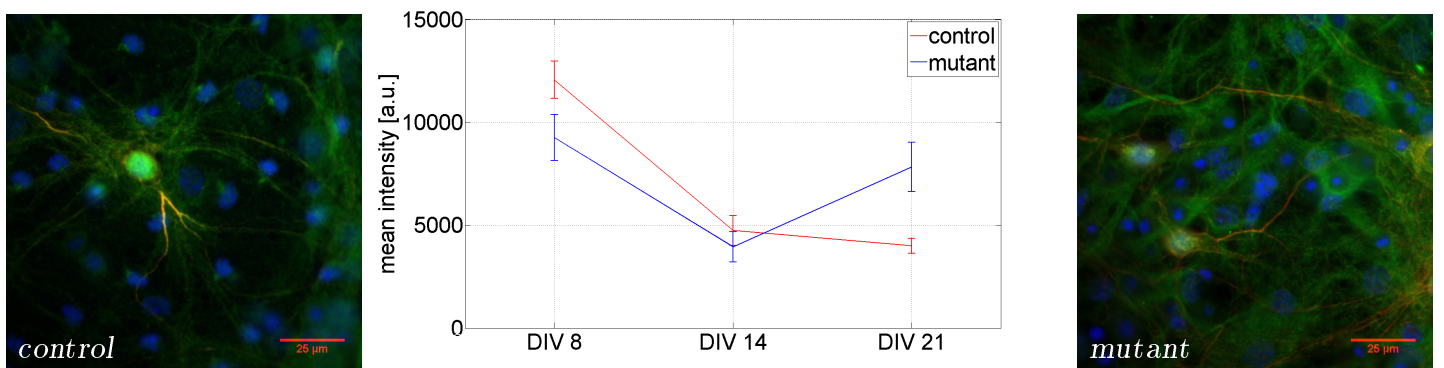

Figure 71: $\mathrm{NrCAM}$ in control and $q v^{3 J}$ mutant neurons. Double labeling of NrCAM (green) and ankG (C-terminal antibody, sc-28561, 11.2 (red) at DIV 21. Nucleus stained by DAPI (blue). Scale bar: $25.0 \mu \mathrm{m}$. Graph: NrCAM expression in control and $q v^{3 J}$ mutant neurons at three maturation stages. The graphic shows the mean fluorescence intensities [a.u.] at the AIS within the distance $0-50.0 \mu \mathrm{m}$ from the soma or first branching point. $\mathrm{N}($ control $)=$ $12,11,12 . \mathrm{N}$ (mutant) $=10,10,9$. The error is the SEM. 


\section{NrCAM organization in wildtypes is regionally periodic.}

The neuronal cell adhesion molecule (NrCAM) binds ankG by a cytoplasmic binding domain (FIGQY) regulated by phosphorylation. 188 The binding of NrCAM to ankG leads to the expectation of a similar organization as found for VGSCs (organized (semi-)periodic). Mainly, NrCAM appears non-periodically organized, only small periodic regions are found. The power spectra are noisy. The mean power spectrum has a small, non-significant power peak at the frequency of about $200 \mathrm{~nm}$. Likely, the large distance between the extracellular labeling positions at the NrCAM molecules and the underlying periodic cytoskeleton does not allow to resolve the underlying periodic structure. A more prominent organization might be obtained by an antibody targeting a cytoplasmic located epitope.

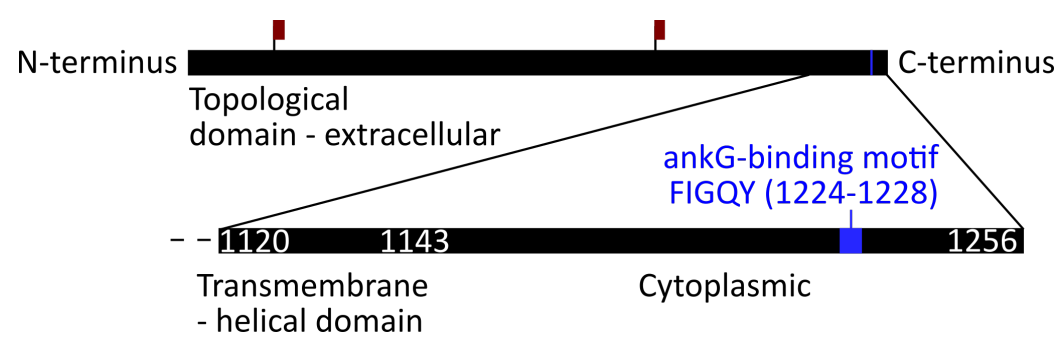

Figure 72: Schematic view of NrCAM isoform1. NrCAM has a large extracellular domain (1120 aa), a small transmembrane domain (23 aa) and a cytoplasmic domain (139 aa). About five sixth of its sequence belongs to the topological domain. The ankG binding motif (aa1224-1228) is located at the cytoplasmic domain. The antibody has been raised against synthetic peptides corresponding to two parts of the topological domain (aa153-174 and aa837-856 (red), published [134] ). White numbers indicate the relative starting and ending amino acids to the three domains. Established after Uniprot.org: Q810U4. 


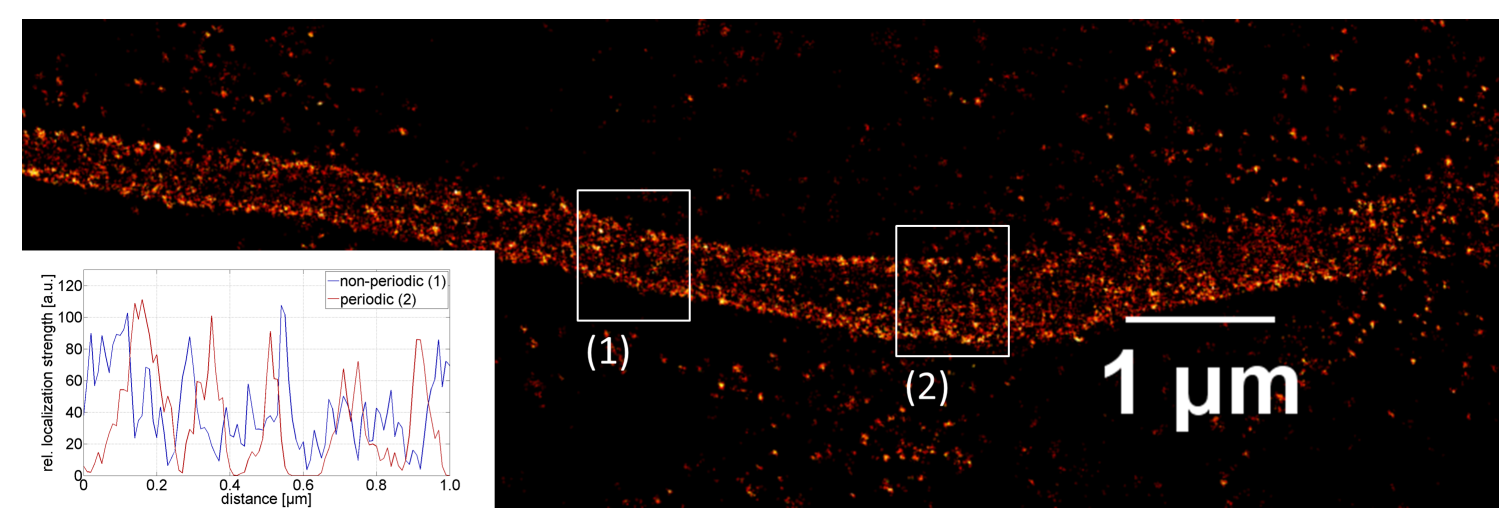

(a) Example AIS: Marked (1) area of non-periodic NrCAM organization. (2) area of periodic NrCAM organization.

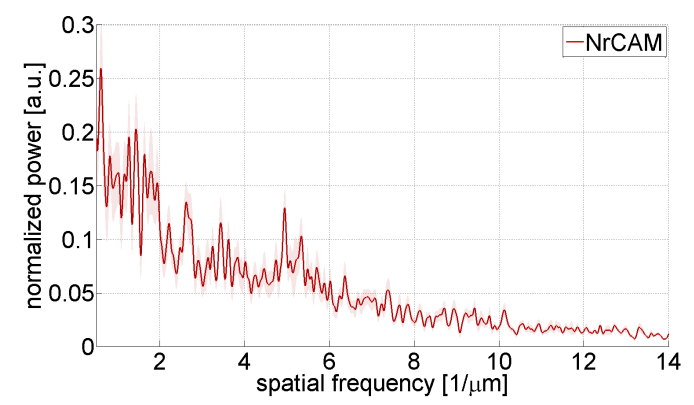

(b) The mean power spectrum calculated from complete

NrCAM data set.

Figure 73: NrCAM in control neurons at the age of DIV 14. (a) Example image of an AIS labeled for NrCAM including marked areas of non-periodic (1) and periodic (2) organization. (b) The mean power spectrum calculated from the complete NrCAM data set. $\mathrm{N}=34$. Error is the SEM. 


\section{Appendix C}

\subsection{Kolmogorov-Smirnov-Test}

The significance of the maturation progress and between control and $q v^{3 J}$ mutant neurons was tested with Kolmogorov-Smirnov-Tests. The tests were performed on the mean fluorescence intensities (without SEM). The significance level is $5.0 \%$. A p-value ( $\mathrm{p}=$ probability for significant difference in \%) equal or greater than $95.0 \%$ is taken as a significant difference between the two tested groups. Otherwise, a p-value below $95.0 \%$ is set as a non-significant difference, the two tested groups are identified as equal or similar.

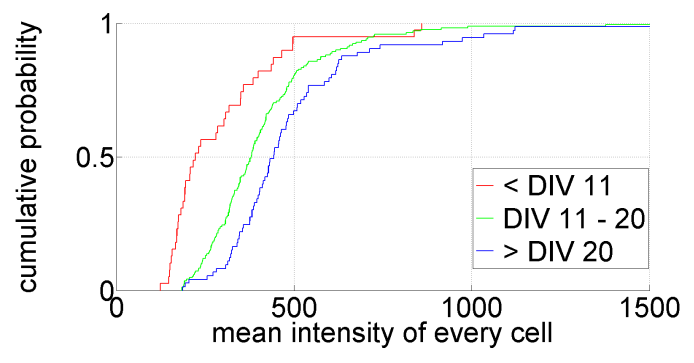

(a) VGSCs

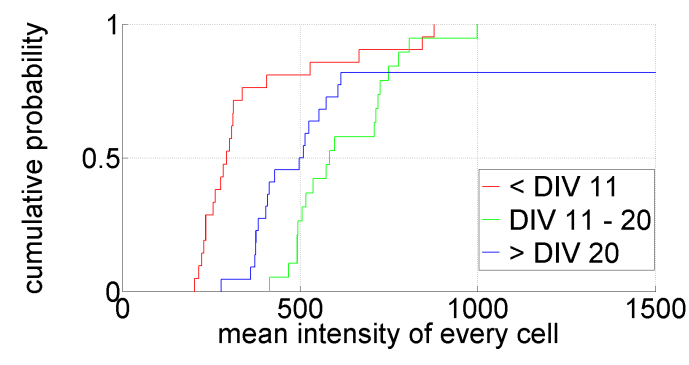

(b) BIV-spectrin labeled N-terminal

Figure 74: Two example graphs of VGSCs and $\beta I V$-spectrin (labeled N-terminal) with maturation in wildtype neurons. Kolmogorov-Smirnov-Tests between groups <DIV 11, DIV 11 - 20 and $>20$. (a) VGSCs, (b) $\beta I V$-spectrin labeled N-terminal. 
The probability of differences between the three maturation stages is summarized in table 1 . In all cases the groups are found to be significantly different, except ankG (C-terminal labeled) within the first two weeks $(<D I V 11, D I V 11-20)$ is found as similar.

Table 1: Significance of matural differences in wildtypes. All three groups were tested against each other for significant differences: p $(<D I V 11, D I V 11-20)$, p $(<D I V 11,>D I V 20)$ and p $(D I V 11-20,>D I V 20)$.

\begin{tabular}{|c|c|c|}
\hline & & in [\%] \\
\hline \multirow[t]{3}{*}{$\beta I V$-spectrin labeled N-terminal } & $\mathrm{p}(<D I V 11, D I V 11-20)$ & $\approx 100.00$ \\
\hline & $\mathrm{p}(<D I V 11,>D I V 20)$ & $\approx 100.00$ \\
\hline & $\mathrm{p}(D I V 11-20,>D I V 20)$ & $\approx 95.30$ \\
\hline \multirow[t]{3}{*}{$\beta I V$-spectrin labeled C-terminal } & $\mathrm{p}(<D I V 11, D I V 11-20)$ & $\approx 100.00$ \\
\hline & $\mathrm{p}(<D I V 11,>D I V 20)$ & $\approx 100.00$ \\
\hline & $\mathrm{p}(D I V 11-20,>D I V 20)$ & $\approx 95.05$ \\
\hline \multirow[t]{3}{*}{ ankG labeled N-terminal } & $\mathrm{p}(<D I V 11, D I V 11-20)$ & $\approx 100.00$ \\
\hline & $\mathrm{p}(<D I V 11,>D I V 20)$ & $\approx 100.00$ \\
\hline & $\mathrm{p}(D I V 11-20,>D I V 20)$ & $\approx 99.77$ \\
\hline \multirow[t]{3}{*}{ ankG labeled C-terminal } & $\mathrm{p}(<$ DIV 11, DIV $11-20)$ & $\approx 80.33$ \\
\hline & $\mathrm{p}(<D I V 11,>D I V 20)$ & $\approx 100.00$ \\
\hline & $\mathrm{p}(D I V 11-20,>D I V 20)$ & $\approx 98.90$ \\
\hline \multirow[t]{3}{*}{ VGSCs } & $\mathrm{p}(<D I V 11$, DIV $11-20)$ & $\approx 100.00$ \\
\hline & $\mathrm{p}(<D I V 11,>D I V 20)$ & $\approx 100.00$ \\
\hline & $\mathrm{p}(D I V 11-20,>D I V 20)$ & $\approx 99.63$ \\
\hline \multirow[t]{3}{*}{$\mathrm{Na}_{\mathrm{v}} 1.6$} & $\mathrm{p}(<D I V 11$, DIV $11-20)$ & $\approx 100.00$ \\
\hline & $\mathrm{p}(<D I V 11,>D I V 20)$ & $\approx 100.00$ \\
\hline & $\mathrm{p}(D I V 11-20,>D I V 20)$ & $\approx 100.00$ \\
\hline \multirow[t]{3}{*}{$\mathrm{Na}_{\mathrm{v}} 1.2$} & $\mathrm{p}(<D I V 11, D I V 11-20)$ & $\approx 100.00$ \\
\hline & $\mathrm{p}(<D I V 11,>D I V 20)$ & $\approx 100.00$ \\
\hline & $\mathrm{p}(D I V 11-20,>D I V 20)$ & $\approx 100.00$ \\
\hline
\end{tabular}


The probability of differences between the three maturation stages is summarized in table 2, For control neurons, in most cases a significant difference is found. Exceptions are ankG (N-terminal labeled) between week two and three and ankG (C-terminal labeled) between week one and two. The maturation in $q v^{3 J}$ mutant neurons appears less clear. For VGSCs no differences are found between the first two weeks of maturation. For ankG (N-terminal labeled) only between the first two weeks a significant difference is identified. For ankG (C-terminal labeled) no significant difference between week one and three is identified.

Table 2: Significance of matural differences in control and $q v^{3 J}$ mutant neurons. All three groups were tested against each other for significant differences: p (<DIV 11, DIV $11-20), \mathrm{p}(<D I V 11,>D I V 20)$ and $\mathrm{p}(D I V$ $11-20,>D I V 20)$.

\begin{tabular}{|l|l|l|l|}
\hline & & control in [\%] & mutant in [\%] \\
\hline VGSCs & $\mathrm{p}(<D I V 11, D I V 11-20)$ & $\approx 99.91$ & $\approx 81.41$ \\
\cline { 2 - 4 } & $\mathrm{p}(<D I V 11,>D I V 20)$ & $\approx 100.00$ & $\approx 95.25$ \\
\cline { 2 - 4 } & $\mathrm{p}(D I V 11-20,>D I V 20)$ & $\approx 99.91$ & $\approx 99.92$ \\
\hline \multirow{5}{*}{ ankG labeled N-terminal } & $\mathrm{p}(<D I V 11, D I V 11-20)$ & $\approx 100.00$ & $\approx 99.45$ \\
\cline { 2 - 4 } & $\mathrm{p}(<D I V 11,>D I V 20)$ & $\approx 100.00$ & $\approx 82.00$ \\
\cline { 2 - 4 } & $\mathrm{p}(D I V 11-20,>D I V 20)$ & $\approx 1.40$ & $\approx 4.23$ \\
\hline ankG labeled C-terminal & $\mathrm{p}(<D I V 11, D I V 11-20)$ & $\approx 100.00$ & $\approx 100.00$ \\
\cline { 2 - 4 } & $\mathrm{p}(<D I V 11,>D I V 20)$ & $\approx 65.86$ & $\approx 27.21$ \\
\cline { 2 - 4 } & $\mathrm{p}(D I V 11-20,>D I V 20)$ & $\approx 99.25$ & $\approx 100.00$ \\
\hline
\end{tabular}


The following table (table: 3) summarizes the probability of differences between control and $q v^{3 J}$ mutant neurons. VGSCs and ankG (C- and N-terminal labeled) show a significant difference. Exception is ankG (C-terminal labeled) at $<D I V$ 11, which reveals no differences between control and $q v^{3 J}$ mutant neurons.

Table 3: Significance of differences between control and $q v^{3 J}$ mutant neurons. Control and $q v^{3 J}$ mutant neurons were tested for significant differences: p (control, DIV $11-20)$, p $(<D I V 11,>D I V 20)$ and p $($ DIV $11-20,>$ DIV 20).

\begin{tabular}{|c|c|c|}
\hline & & $\mathrm{p}$ (control, $\left.q v^{3 J}\right)$ in $[\%]$ \\
\hline \multirow[t]{3}{*}{ VGSCs } & $<D I V 11$ & $\approx 99.93$ \\
\hline & $D I V 11-20$ & $\approx 100.00$ \\
\hline & $>D I V 20$ & $\approx 100.00$ \\
\hline \multirow[t]{3}{*}{ ankG labeled N-terminal } & $<D I V 11$ & $\approx 95.39$ \\
\hline & DIV $11-20$ & $\approx 100.00$ \\
\hline & $>D I V 20$ & $\approx 99.82$ \\
\hline \multirow[t]{3}{*}{ ankG labeled C-terminal } & $<D I V 11$ & $\approx 79.09$ \\
\hline & $D I V 11-20$ & $\approx 100.00$ \\
\hline & $>D I V 20$ & $\approx 94.20$ \\
\hline \multirow[t]{3}{*}{$\mathrm{NrCAM}$} & $<D I V 11$ & $\approx 89.28$ \\
\hline & $D I V 11-20$ & $\approx 99.54$ \\
\hline & $>D I V 20$ & $\approx 96.48$ \\
\hline \multirow[t]{3}{*}{$\beta$ II-spectrin } & $<D I V 11$ & $\approx 63.12$ \\
\hline & DIV $11-20$ & $\approx 42.69$ \\
\hline & $>D I V 20$ & $\approx 77.03$ \\
\hline
\end{tabular}




\subsection{Antibodies}

Primary antibodies were always used with the same secondary antibody for the same project part.

In most cases for wide-field ("2nd antibody wide-field") and STORM ("2nd antibody STORM") imaging different secondaries were applied as noted in the table.

Exceptions are described below.

Table 4: Complete list of primary antibodies and the corresponding secondary antibodies displayed separately for wide-field and STORM imaging.

\begin{tabular}{|c|c|c|c|}
\hline 1st antibody & $\mathrm{c}[\mu \mathrm{g} / \mathrm{mL}]$ & $\begin{array}{l}\text { 2nd antibody } \\
\text { wide-field }\end{array}$ & $\begin{array}{l}\text { 2nd antibody } \\
\text { STORM }\end{array}$ \\
\hline $\begin{array}{l}\text { anti-panNav } \\
\text { monoclonal } \\
\text { Sigma-Aldrich } \\
\text { S8809-1MG } \\
\text { Lot\#: SLBF1242 } \\
\text { and SLBL1663V }\end{array}$ & 1.1 & $\begin{array}{l}\text { anti-mouse (goat) } \\
\text { IgG } \\
\text { Alexa } 488 \\
\text { Molecular Probes } \\
\text { A11029 } \\
\text { Lot\#: } 1252783\end{array}$ & $\begin{array}{l}\text { anti-mouse (goat) } \\
\text { IgG } \\
\text { Alexa } 647 \\
\text { Molecular Probes } \\
\text { A21236 } \\
\text { Lot\#: } 1156622\end{array}$ \\
\hline $\begin{array}{l}\text { anti- } \beta \text { IV-spectrin } \\
\text { polyclonal } \\
\text { Rasband } \\
\text { gift from Rasband } \\
\text { lab, } \\
\text { Baylor College of } \\
\text { Medicine } \\
\text { C-terminal (aa2237- } \\
2253 \text { ) }\end{array}$ & N.A. & $\begin{array}{l}\text { anti-rabbit (goat) } \\
\text { IgG } \\
\text { Alexa } 488 \\
\text { Jackson ImmunoResearch } \\
\text { 111-545-003 } \\
\text { Lot\#: } 107909\end{array}$ & $\begin{array}{l}\text { anti-rabbit (goat) } \\
\text { IgG } \\
\text { Alexa } 647 \\
\text { Molecular Probes } \\
\text { A21245 } \\
\text { Lot\#: } 1219863\end{array}$ \\
\hline $\begin{array}{l}\text { anti- } \beta I V \text {-spectrin } \\
\text { polyclonal } \\
\text { OriGene } \\
\text { TA317365 } \\
\text { Lot\#: } 51117 \\
\text { N-terminal (aa2-14) }\end{array}$ & 2.5 & $\begin{array}{l}\text { anti-goat (donkey) } \\
\text { - IgG } \\
\text { Alexa } 647 \\
\text { Molecular Probes } \\
\text { A21447 } \\
\text { Lot\#: } 1301819\end{array}$ & $\begin{array}{l}\text { anti-goat (donkey) } \\
\text { IgG } \\
\text { Alexa } 647 \\
\text { Molecular Probes } \\
\text { A21447 } \\
\text { Lot\#: } 1301819\end{array}$ \\
\hline $\begin{array}{l}\text { anti- } \beta \text { IV-spectrin } \\
\text { polyclonal } \\
\text { ATLAS } \\
\text { HPA043370 } \\
\text { Lot\#: R39720, } \\
\text { A96519 } \\
\text { central } \\
\text { (aa1142-1235) }\end{array}$ & 1.0 & $\begin{array}{l}\text { anti-rabbit (goat) } \\
\text { IgG } \\
\text { Alexa } 488 \\
\text { Jackson ImmunoResearch } \\
\text { 111-545-003 } \\
\text { Lot\#: } 107909\end{array}$ & $\begin{array}{l}\text { anti-rabbit (goat) } \\
\text { IgG } \\
\text { Alexa } 647 \\
\text { Molecular Probes } \\
\text { A21245 } \\
\text { Lot\#: } 1219863\end{array}$ \\
\hline
\end{tabular}




\begin{tabular}{|c|c|c|c|}
\hline $\begin{array}{l}\text { anti- } \beta \text { IV-spectrin } \\
\text { polyclonal } \\
\text { Santa Cruz } \\
\text { sc-131789 } \\
\text { Lot\#: G0610 } \\
\text { C-terminal (aa2350- } \\
2400 \text { ) }\end{array}$ & 2.5 & $\begin{array}{l}\text { anti-goat (donkey) } \\
\text { IgG } \\
\text { Alexa } 647 \\
\text { Molecular Probes } \\
\text { A21447 } \\
\text { Lot\#: } 1301819\end{array}$ & $\begin{array}{l}\text { anti-goat (donkey) } \\
\mathrm{IgG} \\
\text { Alexa } 647 \\
\text { Molecular Probes } \\
\text { A21447 } \\
\text { Lot\#: } 1301819\end{array}$ \\
\hline $\begin{array}{l}\text { anti-ankG } \\
\text { monoclonal } \\
\text { Santa Cruz } \\
\text { sc-12719 } \\
\text { Lot\#: I0414 } \\
\mathrm{N} \\
\text { terminal, spectrin- } \\
\text { binding domain } \\
\text { (aa982-1107) }\end{array}$ & 1.0 & $\begin{array}{l}\text { anti-mouse (goat) } \\
\text { IgG } \\
\text { Alexa } 488 \\
\text { Molecular Probes } \\
\text { A11029 } \\
\text { Lot\#: } 1252783\end{array}$ & $\begin{array}{l}\text { anti-mouse (goat) } \\
\text { IgG } \\
\text { Alexa } 647 \\
\text { Molecular Probes } \\
\text { A21236 } \\
\text { Lot\#: } 1156622\end{array}$ \\
\hline $\begin{array}{l}\text { anti-ankG } \\
\text { polyclonal } \\
\text { Santa Cruz } \\
\text { sc-28561 } \\
\text { Lot\#: F1511 } \\
\text { C-terminal } \\
\text { (aa4163-4377) }\end{array}$ & 1.0 & $\begin{array}{l}\text { anti-rabbit (goat) } \\
\text { IgG } \\
\text { Alexa } 488 \\
\text { Jackson ImmunoResearch } \\
\text { 111-545-003 } \\
\text { Lot\#: } 107909\end{array}$ & $\begin{array}{l}\text { anti-rabbit (goat) } \\
\text { IgG } \\
\text { Alexa } 647 \\
\text { Molecular Probes } \\
\text { A21245 } \\
\text { Lot\#: } 1219863\end{array}$ \\
\hline $\begin{array}{l}\text { anti-ankG } \\
\text { polyclonal } \\
\text { Santa Cruz } \\
\text { sc-31778 } \\
\text { Lot\#: F0514 } \\
\text { C-terminal } \\
\text { (aa4150-4200) }\end{array}$ & 1.0 & $\begin{array}{l}\text { anti-goat (chicken) } \\
\text { IgG } \\
\text { Alexa } 647 \\
\text { Molecular Probes } \\
\text { A21469 } \\
\text { Lot\#: } 1584272\end{array}$ & $\begin{array}{l}\text { anti-goat (chicken) } \\
\text { IgG } \\
\text { Alexa } 647 \\
\text { Molecular Probes } \\
\text { A21469 } \\
\text { Lot\#: } 1584272\end{array}$ \\
\hline $\begin{array}{l}\text { anti-MAP2 } \\
\text { polyclonal } \\
\text { abcam } \\
\text { ab5392 } \\
\text { Lot\#: G2128901-2 }\end{array}$ & 9.5 & $\begin{array}{l}\text { anti-chicken (goat) } \\
\text { IgG } \\
\text { Alexa } 647 \\
\text { Molecular Probes } \\
\text { A214469 } \\
\text { Lot\#: } 1149008 \\
\text { anti-chicken (donkey) } \\
\text { IgG } \\
\text { Alexa } 488 \\
\text { Jackson ImmunoResearch } \\
\text { 703-545-155 } \\
\text { Lot\#: } 113085\end{array}$ & $\begin{array}{l}\text { anti-chicken (donkey) } \\
\text { IgG } \\
\text { Alexa } 488 \\
\text { Jackson ImmunoResearch } \\
703-545-155 \\
\text { Lot\#: } 113085\end{array}$ \\
\hline
\end{tabular}




\begin{tabular}{|c|c|c|c|}
\hline $\begin{array}{l}\text { anti- } \beta \text { II-spectrin } \\
\text { monoclonal } \\
\text { Santa Cruz } \\
\text { sc-136074 } \\
\text { Lot\#: C2912 }\end{array}$ & 0.5 & $\begin{array}{l}\text { anti-mouse (goat) } \\
\text { IgG } \\
\text { Alexa } 488 \\
\text { Molecular Probes } \\
\text { A11029 } \\
\text { Lot\#: } 1252783 \\
\text { anti-mouse (goat) } \\
\text { IgG } \\
\text { Alexa } 647 \\
\text { Molecular Probes } \\
\text { A21236 } \\
\text { Lot\#: } 1156622\end{array}$ & $\begin{array}{l}\text { anti-mouse (goat) } \\
\text { IgG } \\
\text { Alexa } 647 \\
\text { Molecular Probes } \\
\text { A21236 } \\
\text { Lot\#: } 1156622\end{array}$ \\
\hline $\begin{array}{l}\text { anti-NrCAM } \\
\text { polyclonal } \\
\text { abcam } \\
\text { ab24344 } \\
\text { Lot\#: GR185066-1 }\end{array}$ & N.A. & $\begin{array}{l}\text { anti-rabbit (goat) } \\
\text { IgG } \\
\text { Alexa } 647 \\
\text { Molecular Probes } \\
\text { A21245 } \\
\text { Lot\#: } 1219863\end{array}$ & $\begin{array}{l}\text { anti-rabbit (goat) } \\
\text { IgG } \\
\text { Alexa } 647 \\
\text { Molecular Probes } \\
\text { A21245 } \\
\text { Lot\#: } 1219863\end{array}$ \\
\hline $\begin{array}{l}\text { anti-Na } 1.6 \\
\text { polyclonal } \\
\text { Alomone } \\
\text { ASC-009 } \\
\text { Lot\#: } \\
\text { ASC009AN1650 }\end{array}$ & 4.0 & $\begin{array}{l}\text { anti-rabbit (goat) } \\
\text { IgG } \\
\text { Alexa } 488 \\
\text { Jackson ImmunoResearch } \\
\text { 111-545-003 } \\
\text { Lot\#: } 107909\end{array}$ & $\begin{array}{l}\text { anti-rabbit (goat) } \\
\text { IgG } \\
\text { Alexa } 647 \\
\text { Molecular Probes } \\
\text { A21245 } \\
\text { Lot\#: } 1219863\end{array}$ \\
\hline $\begin{array}{l}\text { anti-Na } 1.6 \\
\text { monoclonal } \\
\text { Neuromab } \\
75-026 \\
\text { Lot\#: 455-2JD-71 }\end{array}$ & 10.0 & $\begin{array}{l}\text { anti-mouse (goat) } \\
\text { IgG } \\
\text { Alexa } 488 \\
\text { Molecular Probes } \\
\text { A11029 } \\
\text { Lot\#: } 1252783\end{array}$ & not performed \\
\hline $\begin{array}{l}\text { anti-Na } 1.6 \\
\text { Rasband } \\
\text { gift from Rasband } \\
\text { lab, } \\
\text { Baylor College of } \\
\text { Medicine }\end{array}$ & N.A. & $\begin{array}{l}\text { anti-rabbit (goat) } \\
\text { IgG } \\
\text { Alexa } 488 \\
\text { Jackson ImmunoResearch } \\
\text { 111-545-003 } \\
\text { Lot\#: } 107909\end{array}$ & not performed \\
\hline $\begin{array}{l}\text { anti-Na } 1.2 \\
\text { monoclonal } \\
\text { Neuromab } \\
\text { SMC-373D } \\
\text { Lot\#: } 1007\end{array}$ & 2.5 & $\begin{array}{l}\text { anti-mouse (goat) } \\
\text { IgG } \\
\text { Alexa } 488 \\
\text { Molecular Probes } \\
\text { A11029 } \\
\text { Lot\#: } 1252783\end{array}$ & $\begin{array}{l}\text { anti-mouse (goat) } \\
\text { IgG } \\
\text { Alexa } 647 \\
\text { Molecular Probes } \\
\text { A21236 } \\
\text { Lot\#: } 1156622\end{array}$ \\
\hline
\end{tabular}




\begin{tabular}{l|l|l|l}
\hline $\begin{array}{l}\text { anti-Na } 1.2 \\
\text { polyclonal }\end{array}$ & 2.0 & anti-rabbit (goat) & anti-rabbit (goat) \\
Alomone & IgG & IgG \\
ASC-002 & Alexa 488 & Alexa 647 \\
Lot\#: & Jackson ImmunoResearch & Molecular Probes \\
ASC002AN1650 & & $111-545-003$ & A21245 \\
& & Lot\#: 107909 & Lot\#: 1219863
\end{tabular}

\section{Exceptions}

Often double labeling with anti-ankG (N- and C-terminal) was performed for an easy AIS identification. For wide-field imaging ankG received an Alexa 488 or Alexa 647 dye depending on which dye was marked as the standard for the protein double labeled with. For STORM imaging ankG received an Alexa 488 (example experiments: 10.3 , 49).

For double labeling of ankG N-terminal and C-terminal received ankG N-terminal an Alexa 647 carrying antibody, whereas the C-terminal received the Alexa 488 carrying antibody (experiments: 9.1.2 For double labeling of anti- $\beta I V-s p e c t r i n$ from OriGene with another primary antibody raised in rabbit, cross-reactions were observed when using anti-rabbit secondaries raised in goat. Consequently, the secondaries were changed to anti-goat (chicken), IgG, Alexa 647, Molecular Probes, A21469, Lot\#: 1584272 and anti-rabbit (donkey), IgG, Alexa 488, abcam, 1584272 (experiments: 9.1.1). 


\section{Bibliography}

[1] Nicholas J Parkinson, Christine L Olsson, Janice L Hallows, Jennifer McKee-Johnson, Bart P Keogh, Konrad Noben-Trauth, Sharon G Kujawa, Bruce L Tempel, "Mutant $\beta$-spectrin 4 causes auditory and motor neuropathies in quivering mice" Nature genetics (2001) 29(1), 61-65.

[2] Ilya A Fleidervish, Nechama Lasser-Ross, Michael J Gutnick, William N Ross, "Na+ imaging reveals little difference in action potential-evoked $\mathrm{Na}+$ influx between axon and soma" Nature neuroscience (2010) 13(7), 852-860.

[3] Maarten HP Kole, Greg J Stuart, "Signal processing in the axon initial segment" Neuron (2012) 73(2), 235-247.

[4] Matthew J Craner, Albert C Lo, Joel A Black, Stephen G Waxman, "Abnormal sodium channel distribution in optic nerve axons in a model of inflammatory demyelination" Brain (2003) 126(7), 1552-1561.

[5] Matthew S Grubb, Juan Burrone, "Building and maintaining the axon initial segment" Current opinion in neurobiology (2010) 20(4), 481-488.

[6] Matthew S Grubb, Yousheng Shu, Hiroshi Kuba, Matthew N Rasband, Verena C Wimmer, Kevin J Bender, "Short-and long-term plasticity at the axon initial segment" The Journal of Neuroscience (2011) 31(45), 16049-16055.

[7] Scott M Jenkins, Vann Bennett, "Ankyrin-G coordinates assembly of the spectrin-based membrane skeleton, voltage-gated sodium channels, and L1 CAMs at Purkinje neuron initial segments" The Journal of cell biology (2001) 155(5), 739-746.

[8] Tatiana Boiko, Audra Van Wart, John H Caldwell, S Rock Levinson, James S Trimmer, Gary Matthews, "Functional specialization of the axon initial segment by isoform-specific sodium channel targeting" The Journal of neuroscience (2003) 23(6), 2306-2313.

[9] Verena C Wimmer, Christopher A Reid, Eva Y-W So, Samuel F Berkovic, Steven Petrou, "Axon initial segment dysfunction in epilepsy" The Journal of physiology (2010) 588(11), $1829-1840$.

[10] Timothy J Byers, Daniel Branton, "Visualization of the protein associations in the 
erythrocyte membrane skeleton" Proceedings of the National Academy of Sciences (1985) 82(18), 6153-6157.

[11] Ke Xu, Guisheng Zhong, Xiaowei Zhuang, "Actin, spectrin, and associated proteins form a periodic cytoskeletal structure in axons" Science (2013) 339(6118), 452-456.

[12] Guisheng Zhong, Jiang He, Ruobo Zhou, Damaris Lorenzo, Hazen P Babcock, Vann Bennett, Xiaowei Zhuang, Robert H Singer, "Developmental mechanism of the periodic membrane skeleton in axons." eLife (2014) 3.

[13] Christophe Leterrier, Jean Potier, Ghislaine Caillol, Fanny Rueda Boroni, Bénédicte Dargent, "Nanoscale architecture of the axon initial segment reveals an organized and robust scaffold" bioRxiv (2015), 022962.

[14] Keith Burridge, Thomas Kelly, Paul Mangeat, "Nonerythrocyte spectrins: actin-membrane attachment proteins occurring in many cell types." The Journal of cell biology (1982) 95(2), 478-486.

[15] Steven R Goodman, Warren E Zimmer, Mary Blair Clark, Ian S Zagon, Jane E Barker, Michael L Bloom, "Brain spectrin: of mice and men" Brain research bulletin (1995) 36(6), 593-606.

[16] Stanny Berghs, Diego Aggujaro, Ronald Dirkx, Elena Maksimova, Paul Stabach, Jean-Michel Hermel, Jian-Ping Zhang, William Philbrick, Vladimir Slepnev, Tatiana Ort et al., " $\beta$ IV spectrin, a new spectrin localized at axon initial segments and nodes of Ranvier in the central and peripheral nervous system" The Journal of cell biology (2000) 151(5), 985-1002.

[17] Masayuki Komada, Philippe Soriano, " $\beta$ IV-spectrin regulates sodium channel clustering through ankyrin-G at axon initial segments and nodes of Ranvier" The Journal of cell biology (2002) 156(2), 337-348.

[18] Sandra Lacas-Gervais, Jun Guo, Nicola Strenzke, Eric Scarfone, Melanie Kolpe, Monika Jahkel, Pietro De Camilli, Tobias Moser, Matthew N Rasband, Michele Solimena, “ $\beta$ IV $\Sigma 1$ spectrin stabilizes the nodes of Ranvier and axon initial segments" The Journal of cell biology (2004) 166(7), 983-990.

[19] Beata Machnicka, Aleksander Czogalla, Anita Hryniewicz-Jankowska, Dżamila M Bogusławska, Renata Grochowalska, Elżbieta Heger, Aleksander F Sikorski, "Spectrins: A structural platform for stabilization and activation of membrane channels, receptors and transporters" Biochimica et Biophysica Acta (BBA)-Biomembranes (2014) 1838(2), 620634. 
[20] P Somogyu, J Hamori, "A quantitative electron microscopic study of the Purkinje cell axon initial segment" Neuroscience (1976) 1(5), 361-IN3.

[21] Debra A Wollner, William A Catterall, "Localization of sodium channels in axon hillocks and initial segments of retinal ganglion cells" Proceedings of the National Academy of Sciences (1986) 83(21), 8424-8428.

[22] Neil A Campbell, Jane B Reece, Jürgen Markl, "Biologie. 6" Aufl. Heidelberg: Spektrum (2003).

[23] Shelley I Fried, Aaron CW Lasker, Neal J Desai, Donald K Eddington, Joseph F Rizzo, "Axonal sodium-channel bands shape the response to electric stimulation in retinal ganglion cells" Journal of neurophysiology (2009) 101(4), 1972-1987.

[24] Matthew S Grubb, Juan Burrone, "Activity-dependent relocation of the axon initial segment fine-tunes neuronal excitability" Nature (2010) 465(7301), 1070-1074.

[25] Matthew N Rasband, "The axon initial segment and the maintenance of neuronal polarity" Nature Reviews Neuroscience (2010) 11(8), 552-562.

[26] Andrea Lorincz, Zoltan Nusser, "Molecular identity of dendritic voltage-gated sodium channels" Science (2010) 328(5980), 906-909.

[27] Zoltan Nusser, "Differential subcellular distribution of ion channels and the diversity of neuronal function" Current opinion in neurobiology (2012) 22(3), 366-371.

[28] Costa M Colbert, Enhui Pan, "Ion channel properties underlying axonal action potential initiation in pyramidal neurons" Nature neuroscience (2002) 5(6), 533-538.

[29] Andrea Lorincz, Zoltan Nusser, "Cell-type-dependent molecular composition of the axon initial segment" The Journal of Neuroscience (2008) 28(53), 14329-14340.

[30] Lucy M Palmer, Greg J Stuart, "Site of action potential initiation in layer 5 pyramidal neurons" The Journal of neuroscience (2006) 26(6), 1854-1863.

[31] Wenqin Hu, Cuiping Tian, Tun Li, Mingpo Yang, Han Hou, Yousheng Shu, "Distinct contributions of Nav1. 6 and Nav1. 2 in action potential initiation and backpropagation" Nature neuroscience (2009) 12(8), 996-1002.

[32] Ekaterini Kordeli, Stephen Lambert, Vann Bennett, "Ankyrin A NEW ANKYRIN GENE WITH NEURAL-SPECIFIC ISOFORMS LOCALIZED AT THE AXONAL INITIAL SEGMENT AND NODE OF RANVIER" Journal of Biological Chemistry (1995) 270(5), 2352-2359. 
[33] James L Salzer, "Polarized domains of myelinated axons" Neuron (2003) 40(2), 297-318.

[34] Kristian L Hedstrom, Xiaorong Xu, Yasuhiro Ogawa, Renato Frischknecht, Constanze I Seidenbecher, Peter Shrager, Matthew N Rasband, "Neurofascin assembles a specialized extracellular matrix at the axon initial segment" The Journal of cell biology (2007) 178(5), $875-886$.

[35] Bettina Winckler, Paul Forscher, Ira Mellman, "A diffusion barrier maintains distribution of membrane proteins in polarized neurons" Nature (1999) $\mathbf{3 9 7}(6721)$, 698-701.

[36] Jürgen-Markus Sobotzik, Jana Maria Sie, Chrisoula Politi, Domenico Del Turco, Vann Bennett, Thomas Deller, Christian Schultz, "AnkyrinG is required to maintain axo-dendritic polarity in vivo" Proceedings of the National Academy of Sciences (2009) 106(41), 1756417569.

[37] Lydia H Davis, Vann Bennett, "Identification of two regions of beta G spectrin that bind to distinct sites in brain membranes." Journal of Biological Chemistry (1994) 269(6), 44094416.

[38] Jyoti Dhar Malhotra, Kristin Kazen-Gillespie, Michael Hortsch, Lori L Isom, "Sodium channel $\beta$ subunits mediate homophilic cell adhesion and recruit ankyrin to points of cell-cell contact" Journal of Biological Chemistry (2000) 275(15), 11383-11388.

[39] Yumi Matsuoka, X Li, Vann Bennett, "Adducin: structure, function and regulation" Cellular and Molecular Life Sciences CMLS (2000) 57(6), 884-895.

[40] David M Shotton, Brian E Burke, Daniel Branton, "The molecular structure of human erythrocyte spectrin: biophysical and electron microscopic studies" Journal of molecular biology (1979) 131(2), 303-329.

[41] Vann Bennett, Daniel Branton, "Selective association of spectrin with the cytoplasmic surface of human erythrocyte plasma membranes. Quantitative determination with purified (32P) spectrin." Journal of Biological Chemistry (1977) 252(8), 2753-2763.

[42] Vann Bennett, Peter J Stenbuck, "Identification and partial purification of ankyrin, the high affinity membrane attachment site for human erythrocyte spectrin." Journal of Biological Chemistry (1979) 254(7), 2533-2541.

[43] Shih-Chun Liu, Laura H Derick, Jiri Palek, "Visualization of the hexagonal lattice in the erythrocyte membrane skeleton." The Journal of cell biology (1987) 104(3), 527-536. 
[44] DW Speicher, Ludmila Weglarz, Tara M DeSilva, "Properties of human red cell spectrin heterodimer (side-to-side) assembly and identification of an essential nucleation site." Journal of Biological Chemistry (1992) 267(21), 14775-14782.

[45] Jonathan Q Davis, Vann Bennett, "Brain ankyrin. A membrane-associated protein with binding sites for spectrin, tubulin, and the cytoplasmic domain of the erythrocyte anion channel." Journal of Biological Chemistry (1984) 259(21), 13550-13559.

[46] Jonathan Q Davis, Tanya McLaughlin, Vann Bennett, "Ankyrin-binding proteins related to nervous system cell adhesion molecules: candidates to provide transmembrane and intercellular connections in adult brain." The Journal of cell biology (1993) 121(1), 121133.

[47] Michael Hortsch, K Sue O'shea, Guangyan Zhao, Feodor Kim, Yolanda Vallejo, Ronald R Dubreuil, "A conserved role for L1 as a transmembrane link between neuronal adhesion and membrane cytoskeleton assembly" Cell Communication and Adhesion (1998) 5(1), 61-73.

[48] Anthony J Baines, "The spectrin-ankyrin-4.1-adducin membrane skeleton: adapting eukaryotic cells to the demands of animal life" Protoplasma (2010) 244(1-4), 99-131.

[49] Elior Peles, James L Salzer, "Molecular domains of myelinated axons" Current opinion in neurobiology (2000) 10(5), 558-565.

[50] VT Marchesi, E Steers, "Selective solubilization of a protein component of the red cell membrane" Science (1968) 159(3811), 203-204.

[51] Nicholas H Putnam, Mansi Srivastava, Uffe Hellsten, Bill Dirks, Jarrod Chapman, Asaf Salamov, Astrid Terry, Harris Shapiro, Erika Lindquist, Vladimir V Kapitonov et al., "Sea anemone genome reveals ancestral eumetazoan gene repertoire and genomic organization" science (2007) 317(5834), 86-94.

[52] Y Yan, E Winograd, A Viel, T Cronin, SC Harrison, D Branton, "Crystal structure of the repetitive segments of spectrin" Science (1993) $\mathbf{2 6 2 ( 5 1 4 2 ) , ~ 2 0 2 7 - 2 0 3 0 . ~}$

[53] AnthonyJ Baines, "Evolution of spectrin function in cytoskeletal and membrane networks" Biochemical Society Transactions (2009) 37(4), 796.

[54] A Viel, MS Gee, L Tomooka, D Branton, "Motifs involved in interchain binding at the tail-end of spectrin" Biochimica et Biophysica Acta (BBA)-Protein Structure and Molecular Enzymology (1998) 1384(2), 396-404. 
[55] WT Tse, MC Lecomte, FF Costa, M Garbarz, C Feo, P Boivin, D Dhermy, BG Forget, "Point mutation in the beta-spectrin gene associated with alpha I/74 hereditary elliptocytosis. Implications for the mechanism of spectrin dimer self-association." Journal of Clinical Investigation (1990) 86(3), 909.

[56] Lisa Cherry, Nick Menhart, Leslie Wo-Mei Fung, "Interactions of the $\alpha$-Spectrin N-terminal Region with $\beta$-Spectrin IMPLICATIONS FOR THE SPECTRIN TETRAMERIZATION REACTION" Journal of Biological Chemistry (1999) 274(4), 2077-2084.

[57] Jonathan J Ipsaro, Sandra L Harper, Troy E Messick, Ronen Marmorstein, Alfonso Mondragón, David W Speicher, "Crystal structure and functional interpretation of the erythrocyte spectrin tetramerization domain complex" Blood (2010) 115(23), 4843-4852.

[58] Ernst UNGEWICKELL, Walter GRATZER, "Self-Association of Human Spectrin" European Journal of Biochemistry (1978) 88(2), 379-385.

[59] Alain Viel, Daniel Branton, "Spectrin: on the path from structure to function" Current opinion in cell biology (1996) 8(1), 49-55.

[60] Vann Bennett, Diana M Gilligan, "The spectrin-based membrane skeleton and micron-scale organization of the plasma membrane" Annual review of cell biology (1993) 9(1), 27-66.

[61] Joacim Robertsson, Katja Petzold, L Lofvenberg, Lars Backman, "Folding of spectrin's SH3 domain in the presence of spectrin repeats" Cellular and Molecular Biology Letters (2005) $\mathbf{1 0}(4), 595$.

[62] Scott P Kennedy, Stephen L Warren, Bernard G Forget, Jon S Morrow, "Ankyrin binds to the 15 th repetitive unit of erythroid and nonerythroid beta-spectrin." The Journal of Cell Biology (1991) 115(1), 267-277.

[63] Jonathan J Ipsaro, Lei Huang, Alfonso Mondragón, "Structures of the spectrin-ankyrin interaction binding domains" Blood (2009) 113(22), 5385-5393.

[64] Yang Yang, Yasuhiro Ogawa, Kristian L Hedstrom, Matthew N Rasband, " $\beta$ IV spectrin is recruited to axon initial segments and nodes of Ranvier by ankyrinG" The Journal of cell biology (2007) 176(4), 509-519.

[65] Yogambal Srinivasan, Lawrence Elmer, Jonathan Davis, Vann Bennett, Kimon Angelides, "Ankyrin and spectrin associate with voltage-dependent sodium channels in brain" Nature (1988). 
[66] Vann Bennett, Stephen Lambert, Jonathan Q Davis, Xu Zhang, "Molecular architecture of the specialized axonal membrane at the node of Ranvier" Society of General Physiologists series (1997) 52, 107-122.

[67] Vann Bennett, Anthony J Baines, "Spectrin and ankyrin-based pathways: metazoan inventions for integrating cells into tissues" Physiological reviews (2001) 81(3), 1353-1392.

[68] Zongming Pan, Tingching Kao, Zsolt Horvath, Julia Lemos, Jai-Yoon Sul, Stephen D Cranstoun, Vann Bennett, Steven S Scherer, Edward C Cooper, "A common ankyrin-Gbased mechanism retains $\mathrm{KCNQ}$ and $\mathrm{NaV}$ channels at electrically active domains of the axon" The Journal of neuroscience (2006) 26(10), 2599-2613.

[69] Helen C Lai, Lily Y Jan, "The distribution and targeting of neuronal voltage-gated ion channels" Nature Reviews Neuroscience (2006) 7(7), 548-562.

[70] David Kapfhamer, Diane E Miller, Stephen Lambert, Vann Bennett, Thomas W Glover, Margit Burmeister, "Chromosomal localization of the ankyrinG gene (ANK3/Ank3) to human 10q21 and mouse 10" Genomics (1995) 27(1), 189-191.

[71] Peter Michaely, Diana R Tomchick, Mischa Machius, Richard GW Anderson, "Crystal structure of a 12 ANK repeat stack from human ankyrinR" The EMBO journal (2002) $\mathbf{2 1}(23), 6387-6396$.

[72] V Bennett et al., "Ankyrins. Adaptors between diverse plasma membrane proteins and the cytoplasm" J. Biol. Chem (1992) 267(13), 8703-8706.

[73] Paul M Jenkins, Namsoo Kim, Steven L Jones, Wei Chou Tseng, Tatyana M Svitkina, Henry H Yin, Vann Bennett, "Giant ankyrin-G: A critical innovation in vertebrate evolution of fast and integrated neuronal signaling" Proceedings of the National Academy of Sciences (2015) 112(4), 957-964.

[74] Peter Michaely, Vann Bennett, "The membrane-binding domain of ankyrin contains four independently folded subdomains, each comprised of six ankyrin repeats." Journal of Biological Chemistry (1993) 268(30), 22703-22709.

[75] Stephen Lambert, Jonathan Q Davis, Vann Bennett, "Morphogenesis of the node of Ranvier: co-clusters of ankyrin and ankyrin-binding integral proteins define early developmental intermediates" The Journal of neuroscience (1997) 17(18), 7025-7036.

[76] Daixing Zhou, Stephen Lambert, Peter L Malen, Scott Carpenter, Linda M Boland, Vann Bennett, "AnkyrinG is required for clustering of voltage-gated Na channels at axon initial 
segments and for normal action potential firing" The Journal of cell biology (1998) 143(5), 1295-1304.

[77] Guy Lemaillet, Barbara Walker, Stephen Lambert, "Identification of a conserved ankyrinbinding motif in the family of sodium channel $\alpha$ subunits" Journal of Biological Chemistry (2003) 278(30), 27333-27339.

[78] Tammy Szu-Yu Ho, Daniel R Zollinger, Kae-Jiun Chang, Mingxuan Xu, Edward C Cooper, Michael C Stankewich, Vann Bennett, Matthew N Rasband, "A hierarchy of ankyrin-spectrin complexes clusters sodium channels at nodes of Ranvier" Nature neuroscience (2014).

[79] Ekaterini Kordeli, Jonathan Davis, Bruce Trapp, Vann Bennett, "An isoform of ankyrin is localized at nodes of Ranvier in myelinated axons of central and peripheral nerves." The Journal of cell biology (1990) 110(4), 1341-1352.

[80] Vann Bennett, Stephen Lambert, "Physiological roles of axonal ankyrins in survival of premyelinated axons and localization of voltage-gated sodium channels" Journal of neurocytology (1999) 28(4-5), 303-318.

[81] Jonathan Q Davis, Stephen Lambert, Vann Bennett, "Molecular composition of the node of Ranvier: identification of ankyrin-binding cell adhesion molecules neurofascin (mucin $+/$ third FNIII domain-) and NrCAM at nodal axon segments." The Journal of cell biology (1996) 135(5), 1355-1367.

[82] Xu Zhang, Vann Bennett, "Restriction of 480/270-kD ankyrin G to axon proximal segments requires multiple ankyrin G-specific domains" The Journal of cell biology (1998) 142(6), 1571-1581.

[83] Wei Chou Tseng, Paul M Jenkins, Masashi Tanaka, Richard Mooney, Vann Bennett, "Giant ankyrin-G stabilizes somatodendritic GABAergic synapses through opposing endocytosis of GABAA receptors" Proceedings of the National Academy of Sciences (2015) 112(4), 12141219.

[84] Anna Brachet, Christophe Leterrier, Marie Irondelle, Marie-Pierre Fache, Victor Racine, Jean-Baptiste Sibarita, Daniel Choquet, Bénédicte Dargent, “Ankyrin G restricts ion channel diffusion at the axonal initial segment before the establishment of the diffusion barrier" The Journal of cell biology (2010) 191(2), 383-395.

[85] Kristian L Hedstrom, Yasuhiro Ogawa, Matthew N Rasband, "AnkyrinG is required for maintenance of the axon initial segment and neuronal polarity" The Journal of cell biology (2008) 183(4), 635-640. 
[86] Dorothy P Schafer, Smita Jha, Fudong Liu, Trupti Akella, Louise D McCullough, Matthew N Rasband, "Disruption of the axon initial segment cytoskeleton is a new mechanism for neuronal injury" The Journal of Neuroscience (2009) 29(42), 13242-13254.

[87] Marie-Pierre Fache, Anissa Moussif, Fanny Fernandes, Pierre Giraud, Juan José Garrido, Bénédicte Dargent, "Endocytotic elimination and domain-selective tethering constitute a potential mechanism of protein segregation at the axonal initial segment" The Journal of cell biology (2004) 166(4), 571-578.

[88] Timothy D Garver, Qun Ren, Shmuel Tuvia, Vann Bennett, "Tyrosine phosphorylation at a site highly conserved in the L1 family of cell adhesion molecules abolishes ankyrin binding and increases lateral mobility of neurofascin" The Journal of cell biology (1997) 137(3), 703-714.

[89] Juan José Garrido, Pierre Giraud, Edmond Carlier, Fanny Fernandes, Anissa Moussif, MariePierre Fache, Dominique Debanne, Bénédicte Dargent, "A targeting motif involved in sodium channel clustering at the axonal initial segment" Science (2003) 300(5628), 2091-2094.

[90] Andreas Gasser, Tammy Szu-Yu Ho, Xiaoyang Cheng, Kae-Jiun Chang, Stephen G Waxman, Matthew N Rasband, Sulayman D Dib-Hajj, "An ankyrinG-binding motif is necessary and sufficient for targeting Nav1. 6 sodium channels to axon initial segments and nodes of Ranvier" The Journal of Neuroscience (2012) 32(21), 7232-7243.

[91] William A Catterall, "Structure and function of voltage-gated sodium channels at atomic resolution" Experimental physiology (2014) 99(1), 35-51.

[92] Helene Vacher, James S Trimmer, "Trafficking mechanisms underlying neuronal voltage-gated ion channel localization at the axon initial segment" Epilepsia (2012) 53(s9), 21-31.

[93] Lori L Isom, KS De Jongh, DE Patton, BF Reber, J Offord, H Charbonneau, K Walsh, AL Goldin, WA Catterall, "Primary structure and functional expression of the beta 1 subunit of the rat brain sodium channel" Science (1992) 256(5058), 839-842.

[94] Alan L Goldin, Robert L Barchi, John H Caldwell, Franz Hofmann, James R Howe, John C Hunter, Roland G Kallen, Gail Mandel, Miriam H Meisler, Yoheved Berwald Netter et al., "Nomenclature of voltage-gated sodium channels" Neuron (2000) 28(2), 365-368.

[95] Ning Qin, Michael R D’Andrea, Mary-Lou Lubin, Navid Shafaee, Ellen E Codd, Ana M Correa, "Molecular cloning and functional expression of the human sodium channel $\beta 1 \mathrm{~B}$ subunit, a novel splicing variant of the $\beta 1$ subunit" European journal of biochemistry (2003) 270(23), 4762-4770. 
[96] Frank H Yu, Ruth E Westenbroek, Inmaculada Silos-Santiago, Kimberly A McCormick, Deborah Lawson, Pei Ge, Holly Ferriera, Jeremiah Lilly, Peter S DiStefano, William A Catterall et al., "Sodium channel beta4, a new disulfide-linked auxiliary subunit with similarity to beta2." The Journal of neuroscience: the official journal of the Society for Neuroscience (2003) 23(20), 7577-7585.

[97] John H Caldwell, Kristin L Schaller, Robert S Lasher, Elior Peles, S Rock Levinson, "Sodium channel Nav1. 6 is localized at nodes of Ranvier, dendrites, and synapses" Proceedings of the National Academy of Sciences (2000) 97(10), 5616-5620.

[98] Matthew N Rasband, James S Trimmer, "Developmental clustering of ion channels at and near the node of Ranvier" Developmental biology (2001) 236(1), 5-16.

[99] Tatiana Boiko, Matthew N Rasband, S Rock Levinson, John H Caldwell, Gail Mandel, James S Trimmer, Gary Matthews, "Compact myelin dictates the differential targeting of two sodium channel isoforms in the same axon" Neuron (2001) 30(1), 91-104.

[100] Charlotte F Ratcliffe, Ruth E Westenbroek, Rory Curtis, William A Catterall, "Sodium channel $\beta 1$ and $\beta 3$ subunits associate with neurofascin through their extracellular immunoglobulin-like domain" The Journal of cell biology (2001) 154(2), 427-434.

[101] Matthew J Craner, Jia Newcombe, Joel A Black, Caroline Hartle, M Louise Cuzner, Stephen G Waxman, "Molecular changes in neurons in multiple sclerosis: altered axonal expression of Nav1. 2 and Nav1. 6 sodium channels and $\mathrm{Na}+/ \mathrm{Ca} 2+$ exchanger" Proceedings of the National Academy of Sciences of the United States of America (2004) 101(21), 81688173.

[102] Miriam H Meisler, Nicholas W Plummer, Daniel L Burgess, David A Buchner, Leslie K Sprunger, "Allelic mutations of the sodium channel SCN8A reveal multiple cellular and physiological functions", In Mutagenesis of the Mouse Genome, 37-45. Springer (2004).

[103] Miriam H Meisler, Jennifer Kearney, Andrew Escayg, Bryan T Macdonald, Leslie K Sprunger, "Sodium channels and neurological disease: insights from Scn8a mutations in the mouse" The Neuroscientist (2001) 7(2), 136-145.

[104] CH Yoon, EP Les, "Quivering, a new first chromosome mutation in mice" Journal of Heredity (1957) 48(4), 176-180.

[105] Gregory R Bock, Marius P Frank, Karen P Steel, MS Deol, "The quivering mutant mouse: hereditary deafness of central origin" Acta oto-laryngologica (1983) 96(5-6), 371-377. 
[106] MS Deol, Marius P Frank, Karen P Steel, Gregory R Bock, "Genetic deafness of central origin" Brain research (1983) 258(1), 177-179.

[107] Yang Yang, Sandra Lacas-Gervais, D Kent Morest, Michele Solimena, Matthew N Rasband, " $\beta$ IV spectrins are essential for membrane stability and the molecular organization of nodes of Ranvier" The Journal of neuroscience (2004) 24(33), 7230-7240.

[108] Michael J Rust, Mark Bates, Xiaowei Zhuang, "Sub-diffraction-limit imaging by stochastic optical reconstruction microscopy (STORM)" Nature methods (2006) 3(10), 793-796.

[109] Ernst Abbe, "Beiträge zur Theorie des Mikroskops und der mikroskopischen Wahrnehmung" Archiv für mikroskopische Anatomie (1873) 9(1), 413-418.

[110] George Patterson, Michael Davidson, Suliana Manley, Jennifer Lippincott-Schwartz, "Superresolution imaging using single-molecule localization" Annual review of physical chemistry (2010) 61, 345 .

[111] Sebastian van de Linde, Anna Löschberger, Teresa Klein, Meike Heidbreder, Steve Wolter, Mike Heilemann, Markus Sauer, "Direct stochastic optical reconstruction microscopy with standard fluorescent probes" Nature protocols (2011) 6(7), 991-1009.

[112] Ulrike Endesfelder, Sebastian Malkusch, Benjamin Flottmann, Justine Mondry, Piotr Liguzinski, Peter J Verveer, Mike Heilemann, "Chemically induced photoswitching of fluorescent probes-a general concept for super-resolution microscopy" Molecules (2011) 16(4), 3106-3118.

[113] Jie Wang, David A Richards, "Segregation of PIP2 and PIP3 into distinct nanoscale regions within the plasma membrane" Biology open (2012) 1(9), 857-862.

[114] Xiaowei Zhuang, "Nano-imaging with STORM" Nature photonics (2009) 3(7), 365.

[115] Graham T Dempsey, Joshua C Vaughan, Kok Hao Chen, Mark Bates, Xiaowei Zhuang, "Evaluation of fluorophores for optimal performance in localization-based super-resolution imaging" Nature methods (2011) 8(12), 1027-1036.

[116] Peter Wardman, "Reduction potentials of one-electron couples involving free radicals in aqueous solution" Journal of Physical and Chemical Reference Data (1989) 18(4), 16371755 .

[117] Mike Heilemann, Peter Dedecker, Johan Hofkens, Markus Sauer, "Photoswitches: Key molecules for subdiffraction-resolution fluorescence imaging and molecular quantification" Laser E Photonics Reviews (2009) 3(1-2), 180-202. 
[118] Sebastian van de Linde, Ivan Krstić, Thomas Prisner, Sören Doose, Mike Heilemann, Markus Sauer, "Photoinduced formation of reversible dye radicals and their impact on superresolution imaging" Photochemical $\&$ S Photobiological Sciences (2011) 10(4), 499-506.

[119] Bo Huang, Hazen Babcock, Xiaowei Zhuang, "Breaking the diffraction barrier: superresolution imaging of cells" Cell (2010) $\mathbf{1 4 3 ( 7 ) , ~ 1 0 4 7 - 1 0 5 8 . ~}$

[120] Nick Goeden, Alexandre Bonnin, "Ex vivo perfusion of mid-to-late-gestation mouse placenta for maternal-fetal interaction studies during pregnancy" Nature protocols (2013) 8(1), 66-74.

[121] Gerard MJ Beaudoin III, Seung-Hye Lee, Dipika Singh, Yang Yuan, Yu-Gie Ng, Louis F Reichardt, Jyothi Arikkath, "Culturing pyramidal neurons from the early postnatal mouse hippocampus and cortex" Nature protocols (2012) 7(9), 1741-1754.

[122] Steve Wolter, "rapid STORM manual" http://www.super-resolution.de/home/rapidstorm/ (2012).

[123] William RJ Whitaker, Richard LM Faull, Henry J Waldvogel, Christopher J Plumpton, Piers C Emson, Jeffrey J Clare, "Comparative distribution of voltage-gated sodium channel proteins in human brain" Molecular brain research (2001) 88(1), 37-53.

[124] Andrea Lorincz, Zoltan Nusser, "Specificity of immunoreactions: the importance of testing specificity in each method" The Journal of neuroscience (2008) 28(37), 9083-9086.

[125] Jean-Marc Fritschy, "Is my antibody-staining specific? How to deal with pitfalls of immunohistochemistry" European Journal of Neuroscience (2008) 28(12), 2365-2370.

[126] Matthew N Rasband, "Cytoskeleton: axons earn their stripes" Current Biology (2013) 23(5), R197-R198.

[127] Beat M Riederer, Ian S Zagon, Steven R Goodman, "Brain spectrin (240/235) and brain spectrin $(240 / 235 \mathrm{E})$ : two distinct spectrin subtypes with different locations within mammalian neural cells." The Journal of cell biology (1986) 102(6), 2088-2097.

[128] Christian R Lombardo, Scott A Weed, Scott P Kennedy, Bernard G Forget, Jon S Morrow, "Beta II-spectrin (fodrin) and beta I epsilon 2-spectrin (muscle) contain NH2-and COOH-terminal membrane association domains (MAD1 and MAD2)." Journal of Biological Chemistry (1994) 269(46), 29212-29219.

[129] Libo Yao, Paul Janmey, Luciano G Frigeri, Wei Han, Jun Fujita, Yuko Kawakami, John R Apgar, Toshiaki Kawakami, "Pleckstrin homology domains interact with filamentous actin" Journal of Biological Chemistry (1999) 274(28), 19752-19761. 
[130] G Elisabeth Pollerberg, Keith Burridge, Keith E Krebs, Steven R Goodman, Melitta Schachner, "The $180-\mathrm{kD}$ component of the neural cell adhesion molecule N-CAM is involved in cell-cell contacts and cytoskeleton-membrane interactions" Cell and tissue research (1987) $\mathbf{2 5 0}(1), 227-236$.

[131] Lisa M Sharkey, Xiaoyang Cheng, Valerie Drews, David A Buchner, Julie M Jones, Monica J Justice, Stephen G Waxman, Sulayman D Dib-Hajj, Miriam H Meisler, "The ataxia3 mutation in the N-terminal cytoplasmic domain of sodium channel Nav1. 6 disrupts intracellular trafficking" The Journal of Neuroscience (2009) 29(9), 2733-2741.

[132] Osamu Ohara, Reiko Ohara, Hisashi Yamakawa, Daisuke Nakajima, Manabu Nakayama, "Characterization of a new $\beta$-spectrin gene which is predominantly expressed in brain" Molecular brain research (1998) 57(2), 181-192.

[133] Yasuhiro Ogawa, Matthew N Rasband, "The functional organization and assembly of the axon initial segment" Current opinion in neurobiology (2008) 18(3), 307-313.

[134] Martin Grumet, Vincent Mauro, Mark P Burgoon, Gerald M Edelman, Bruce A Cunningham, "Structure of a new nervous system glycoprotein, Nr-CAM, and its relationship to subgroups of neural cell adhesion molecules." The Journal of cell biology (1991) 113(6), 1399-1412. 


\section{List of Figures}

1 The hippocampal neuron. . . . . . . . . . . . . . . . . . . 6

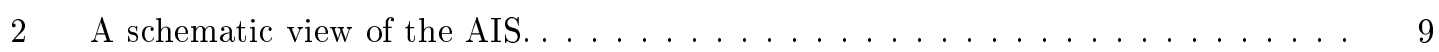

$3 \quad$ A schematic view of the $\beta I V$-spectrin isoforms. . . . . . . . . . . . . . . 10

$4 \quad$ A schematic view of the giant $480 \mathrm{kDa}$ and the $270 \mathrm{kDa}$ ankG isoforms. . . . . . . . 12

5 Conserved sequence of ankG binding motifs in sodium channel subtypes. . . . . . . 14

6 Quivering - new extension in $\beta$ IV-spectrin. . . . . . . . . . . . . . . 15

$7 \quad$ Schematic resolution of wide-field versus STORM microscopy. . . . . . . . . . . . . 15

8 The concept of STORM imaging. . . . . . . . . . . . . . . . . . 16

$9 \quad$ Photoswitching of Alexa dyes. . . . . . . . . . . . . . . . . . . 17

10 Genotyping results: $q v^{3 J}$ mutant. . . . . . . . . . . . . . . . . . . 20

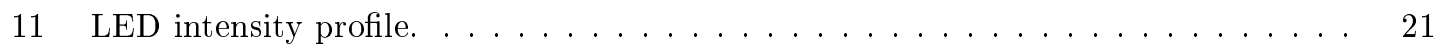

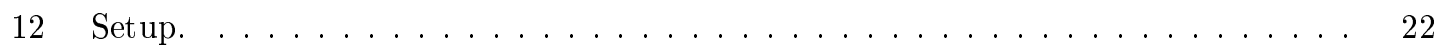

13 Framerates. . . . . . . . . . . . . . . . . . . . . . 23

14 Drift analysis. . . . . . . . . . . . . . . . . . . . . . . 24

15 Example of analysis of wide-field images. . . . . . . . . . . . . . . . 26

16 Example for analysis of STORM images. . . . . . . . . . . . . . . . . . . 27

17 Fourier transformation. . . . . . . . . . . . . . . . . . . . . . . . . 28

18 Example images. $\beta I V-$-spectrin labeled at its N-terminus in development. . . . . . . 32

$19 \quad$ Example images. $\beta$ IV-spectrin labeled at its C-terminus in development. . . . . . . 32

$20 \mathrm{BIV-spectrin} \mathrm{in} \mathrm{development.} \mathrm{.} \mathrm{.} \mathrm{.} \mathrm{.} \mathrm{.} \mathrm{.} \mathrm{.} \mathrm{.} \mathrm{.} \mathrm{.} \mathrm{.} \mathrm{.} \mathrm{.} \mathrm{.} \mathrm{.} \mathrm{.} \mathrm{.} \mathrm{.} \mathrm{.} 32$

21 Example images. AnkG labeled at its C- and N-terminus. . . . . . . . . . . . . 33

22 AnkG in development. . . . . . . . . . . . . . . . . . . . . 33

$23 \quad$ Example images. Voltage-gated sodium channels in development. . . . . . . . . . . 34

24 Voltage-gated sodium channels in development. . . . . . . . . . . . . . . . . . 34

25 Normalization: VGSC position in development. . . . . . . . . . . . . . . . 35

26 Normalization: VGSC and $\beta I V-$ spectrin colocalization in maturation. . . . . . . . . 35

27 Sodium channel density in wildtypes with maturation. . . . . . . . . . . . 36

28 Example images. Voltage-gated sodium channel subtype $\mathrm{Na}_{\mathrm{v}} 1.6$ in development. . 37

$29 \quad$ Voltage-gated sodium channel subtype $\mathrm{Na}_{\mathrm{v}} 1.6$ in development. . . . . . . . . . . . . 37

$30 \quad$ Example images. Voltage-gated sodium channel subtype $\mathrm{Na}_{\mathrm{v}} 1.2$ in development. . 38

31 Sodium channel subtype $\mathrm{Na}_{\mathrm{v}} 1.2$ in development. . . . . . . . . . . . . . . . . 38

$32 \quad \beta I V-s p e c t r i n$ organization in the AIS imaged by STORM at DIV 11 - 20. . . . . . 45 
$33 \quad \beta I V-s p e c t r i n$ organization in the AIS of immature neurons imaged by STORM at

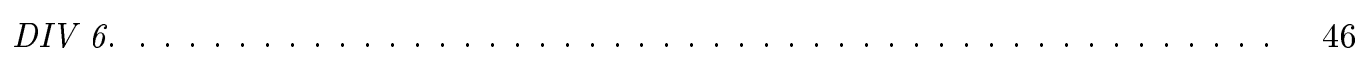

$34 \quad$ AnkG organization in the AIS imaged by STORM $D I V 11-20 . \ldots \ldots 4$

$35 \quad$ VGSC organization in the AIS imaged by STORM DIV $11-20 . \ldots \ldots$

36 Model: $A$ schematic view of anti- $\beta$ IV-spectrin antibodies binding to the $\beta \mathrm{IV}$ -

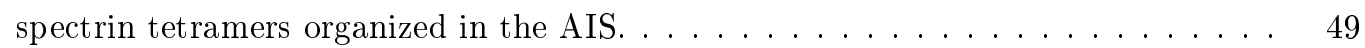

37 Model of $\beta$ IV-spectrin organization in the AIS. . . . . . . . . . . . . 50

38 Model: A schematic view of the $\beta I V-$ spectrin tetramer and its binding sites to ankG. 52

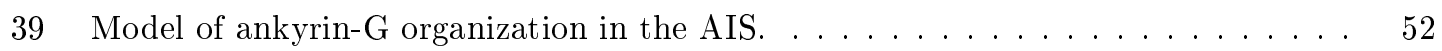

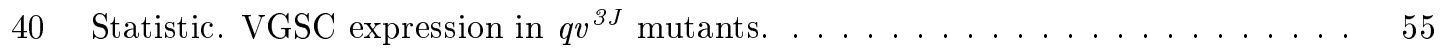

41 Statistic. VGSC subtype expression in $q v^{3 J}$ mutants. . . . . . . . . . . . 56

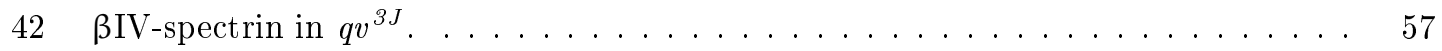

$43 \quad$ BIV-spectrin in development in control neurons. . . . . . . . . . . . . . . . 57

44 Example images. AnkG in development in control and $q v^{3 J}$ mutant neurons. . . . 58

$45 \quad$ AnkG in development in control and $q v^{3 J}$ mutant neurons. . . . . . . . . . 58

$46 \quad$ Example images. Voltage-gated sodium channels in development in control and $q v^{3 J}$

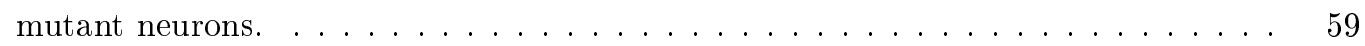

$47 \quad$ Voltage-gated sodium channels in development in control and $q v^{3 J}$ mutant neurons. 59

$48 \quad$ Sodium channel density in $q v^{3 J}$ mutant and control neurons with maturation. . . . $\quad 60$

$49 \quad$ Example images. Sodium channels in $q v^{3 J}$ mutant neurons. . . . . . . . . . . . . 64

$50 \quad$ Structural comparison of $\beta I V$-spectrin in control and $q v^{3 J}$ mutant neurons at the age $<$ DIV 11 by STORM imaging. . . . . . . . . . . . . . . . . . 66

$51 \quad$ Structural comparison of ankG in control and $q v^{3 J}$ mutant neurons in development by STORM imaging. . . . . . . . . . . . . . . . . . . . . . 67

52 Structural comparison of ankG in control and $q v^{3 J}$ mutant neurons by STORM imaging at $D I V 24 . \ldots \ldots \ldots \ldots \ldots \ldots$

$53 \quad$ Structural comparison of VGSCs in control and $q v^{3 J}$ mutant neurons in development by STORM imaging. . . . . . . . . . . . . . . . . . . . . . . 69

54 Model: Potential ankG binding partners. . . . . . . . . . . . . . . . 71

$55 \quad$ Extended model of AIS proteins. . . . . . . . . . . . . . . . . . 75

56 A schematic view of the $\beta I V$-spectrin isoforms. Antibody binding positions. . . . $\quad 76$

$57 \quad$ Example images. $\beta I V-$ spectrin double labeling. . . . . . . . . . . . . . . . . . 78

$58 \quad$ Example images of $\beta I V-$ spectrin central antibody artefacts. . . . . . . . . . . . 78

$59 \quad \beta I V-s p e c t r i n$ double labeling in development. Double labeling of N-terminal and

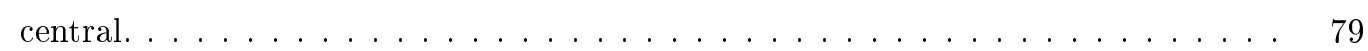


$60 \quad$ BIV-spectrin double labeling in development. Double labeling of N- and C-terminal. 79

61 Example images of ankG double labeling. . . . . . . . . . . . . . . . . . . . 80

$62 \quad$ AnkG double labeling in development. . . . . . . . . . . . . . . . . . . . . 81

63 Comparison of three different anti-Na $\mathrm{Na}_{\mathrm{v}} 1.6$ antibodies. . . . . . . . . . . . . . . 82

64 Comparison of two different anti- $\mathrm{Na}_{\mathrm{v}} 1.2$ antibodies. . . . . . . . . . . . . . 84

$65 \quad$ BII-spectrin in development in control and $q v^{3 J}$ mutant neurons. . . . . . . . . . . 85

66 Structural organization of $\beta$ II-spectrin in neurites. . . . . . . . . . . . . . . 86

67 Structural comparison of $\beta I I-s p e c t r i n$ in control and $q v^{3 J}$ mutant neurons. . . . . . 87

68 Model of ankyrin-G organization in the AIS. . . . . . . . . . . . . . . . . 89

69 Structural comparison of ankG (labeled C-terminal) in control and $q v^{3 J}$ mutant neurons by STORM imaging. . . . . . . . . . . . . . . . . . 90

$70 \quad$ Details of $\mathrm{Na}_{\mathrm{v}} 1.2$ structural organization. . . . . . . . . . . . . . . . . 91

$71 \quad \mathrm{NrCAM}$ in control and $q v^{3 J}$ mutant neurons. . . . . . . . . . . . . . . . . 92

$72 \quad$ Schematic view of the main NrCAM isoform $1 . \ldots \ldots \ldots$. . . . . . . . . 93

73 Structural imaging of NrCAM. . . . . . . . . . . . . . . . . . 94

$74 \quad$ Example graphs for Kolmogorov-Smirnov-Tests. . . . . . . . . . . . . . . . . . 95 


\section{List of Tables}

$1 \quad$ Significance of matural differences in wildtypes. $\ldots \ldots \ldots \ldots \ldots$

$2 \quad$ Significance of matural differences in control and $q v^{3 J}$ mutant neurons. . . . . . . $\quad 97$

$3 \quad$ Significance of differences between control and $q v^{3 J}$ mutant neurons. . . . . . . . 98

$4 \quad$ Antibody list. . . . . . . . . . . . . . . . . . . . . . . . . . 99 


\section{Acronyms}

aa

amino acid

ABD actin-binding domain

AIS axon initial segment

ankB ankyrin-B

ankG ankyrin-G

AP action potential

a.u. arbitrary units

bfgf basic fibroblast growth factor

bp base pairs

BSA bovine serum albumin

CNS central nervous system

DAPI 4',6-Diamidin-2-phenylindol

dATP desoxyadenosintriphosphat

dCTP desoxycytidintriphosphat

dGTP desoxyguanosintriphosphat

DIV days in vitro

dTTP desoxythymidintriphosphat

DNA deoxyribonucleic acid

E18 embryonic day 18

EDTA ethylenediaminetetraacetic acid

EMCCD electron multiplying charge-coupled device

FWHM full width at half maximum

HILO highly inclined thin illumination

LED light-emitting diode 
MAP2 microtubule-associated protein 2

MEA cysteamine hydrochloride

MEM Minimum Essential Media

NA numerical aperture

$\mathrm{NaOH} \quad$ sodium hydroxide

Nav sodium channels

NF neurofilament

noR nodes of Ravier

NrCAM neuronal cell adhesion molecule

PCR polymerase chain reaction

P0 postnatal day 0 (newborn)

P2 postnatal day 2

PBS phosphate buffered saline

PCs purkinje cells

PH-domain pleckstrin homology domain

PNS pheriperal nervous system

PSF point spread function

qv quivering

RNA ribonucleic acid

SDS sodium dodecyl sulfate

SEM standard error of the mean

shRNA small hairpin ribonucleic acid

SH3 Src-homology 3

STORM stochastic optical reconstruction microscopy

TIRF total internal reflection fluorescence 
TRIS tris(hydroxymethyl)-aminomethan

UV ultraviolet

VGSCs voltage-gated sodium channels 


\section{Acknowledgments}

Supervisior: Jörg Enderlein and Andreas Neef.

Thesis Committee: Jörg Enderlein, Andreas Neef, Kai Tittmann, Eberhard Bodenschatz.

Thesis project was performed in collaboration with Elinor Lazarov, Hebrew University of Jerusalem, Israel. She performed the electrophysiology measurements, as noted above, and provided the immunohistochemistry protocol.

C57BL/6N mice were obtained from the Tierhaus, Max Planck Institute for Experimental Medicine, Göttingen.

$Q v^{3 J}$ mutant mice were provided by Dr. Nicola Strenzke and Gulnara Yamanbaeva, InnerEarLab, The University Medical Center Göttingen. Breeding of $q v^{3 J}$ mutants was performed by the animal caretaker Daniela Marschall, Zentrale Tierexperimentelle Einrichtung (ZTE), The University Medical Center Göttingen.

Genotyping of $q v^{3 J}$ mutants was performed by the technical assistants Sandra Gerke and Christiane Senger-Freitag, InnerEarLab, The University Medical Center Göttingen.

Protocols for hippocampal neuron dissection, cultivation and general assistance for troubleshooting were obtained from the technical assistant Sabine Klöppner, Max-Planck Institute for Experimental Medicine.

A C-terminal $\beta I V$-spectrin and a subtype $\mathrm{Na}_{\mathrm{v}} 1.6$ antibody were provided by Rasband lab, Baylor College of Medicine.

Help with orders and general lab assistance was obtained from the technical assistant Stefanie Kramer, The University of Göttingen.

Questions regarding STORM imaging were kindly answered by Steve Wolter and Dr. Sebastian van der Linde. 


\section{Curriculum vitae}

Melanie Dannemeyer

Date of birth: 24. Juli 1986

Place of birth: Bremen

Nationality: German

\section{Education}

$12 / 2012-$

$10 / 2010-09 / 2012$

University and Arizona State University:

Master thesis in Biophysics, Arizona State University:

"Biophysical Characterization of Cancer Cells

by Combined Atomic Force Microscopy -

Confocal Fluorescence Microscopy"

Supervisor: Prof. Dr. Robert Ros, Prof. Armin Gölzhäuser

10/2007 - 09/2010 Bachelor of Science in Molecular Biotechnology, Bielefeld University

08/1999 - 06/2006 High School - Gymnasium Brake

\section{International Experience}

12/2011 - 07/2012 Visiting Student, Robert Ros Lab, Center for Biological Physics, Department of Physics, Arizona State University, AZ.

09/2011 - 12/2011 Visiting Student, Alexandra Ros Lab, Department of Chemistry and Biochemistry, Arizona State University, AZ.

08/2003 - 05/2004 Student Exchange Year, South Hills High School, West Covina, CA.

\section{Publications}

Doss, B. L., Staunton, J. R., Dannemeyer, M., Linhart, M., \& Ros, R. (2012). "Combined AFM Nanoindentation and Finite Element Analysis of Soft Heterogeneous Biomaterials", Microscopy and Microanalysis, 18(S2), 866-867. 


\section{Conferences}

\section{Talks}

"Combined Atomic Force and Confocal Fluorescence Microscopy for Elasticity Measurements of Living Cells", Oral presentation at Biophest, University of Arizona, Tucson, 2012.

\section{Posters}

"Structural changes in the axon initial segment of developing neurons and qv3J mutants", Axons in the desert II, Israel, 2015.

"Imaging of VGSC Alteration in Cultured Hippocampal Neurons", 58th Annual Meeting of the Biophysical Society, San Francisco, 2014.

"Super-resolution Cryo-fluorescence Microscopy", Visit IMPRS Physics of Biological and Complex Systems, MPI for Biophysical Chemistry, Göttingen, 2013.

\section{Scholarships}

Rectorate Scholarship Bielefeld University, 2011.

PROMOS Scholarship for study abroad (DAAD), 2011-2012.

\section{Miscellaneous}

Trainer for Pre- and Postnatal Training, 05/2015.

Trainer for Muscle - Activity - Excellence, 03/2015.

Trainer for Indoor Cycling, 12/2014.

Group Fitness B-License at BSA-Academy, School of Health Management, 09/2014. 